Abstracts ${ }^{*}$

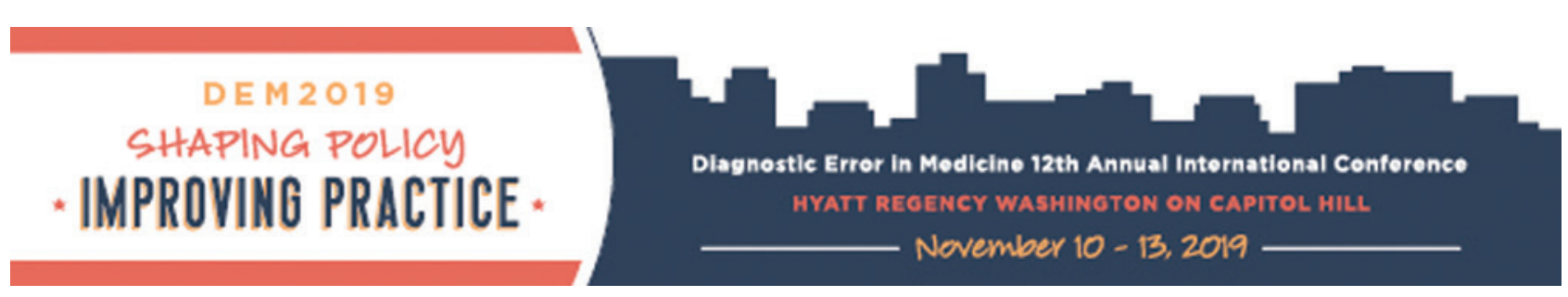

\title{
The Diagnostic Error in Medicine
}

\section{2th Annual International Conference}

\author{
November 10-13, 2019 \\ Washington, D.C. \\ Planning Committee \\ Michael Kanter, MD | Chair \\ Leslie Tucker | Co-Chair \\ Jeff Brady, MD, MPH \\ Elizabeth Cohen, MPH \\ Robert El-Kareh, MD, MPH, MS \\ Kelly Gleason, RN, PhD \\ Kathryn McDonald, MM, PhD \\ Andrew Olson, MD \\ Ruth Ryan, RN, BSN, MSW, CPHRM \\ Kathryn Schaefer, MSN, RN, CPHRM \\ Sue Sheridan, MBA, MIM, DHL \\ Lisa Simpson, MB, BCh, MPH, FAAP
}

\author{
Abstract Selection Committee \\ Christina Cifra, MD, MS \\ Janice Kwan, MD, MPH \\ Benji Mathews, MD \\ Ashley Meyer, $\mathrm{PhD}$
}

${ }^{*}$ These abstracts have been reproduced directly from the material supplied by the authors, without editorial alteration by the staff of this Journal. Insufficiencies of preparation, grammar, spelling, style, syntax and usage are the authors' responsibility. 


\title{
Best of the Best Oral Abstract Plenary
}

\author{
Wednesday, November 13 \\ 8:15 AM - 10:00 AM
}

\section{Communication Failures as Contributors to Diagnostic Errors: Implications for the Care of Latino Communities}

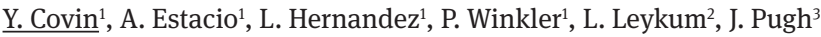 \\ ${ }^{1}$ UT Health San Antonio, San Antonio, TX \\ ${ }^{2}$ UT Health San Antonio and Audie L. Murphy VA Health System, San Antonio, TX \\ ${ }^{3}$ Audie L. Murphy VA Health System, San Antonio, TX
}

Background: The prevailing theory regarding disparities in diagnostic errors in primary care suggests implicit bias, and clinical reasoning as the major causal factors. This view is limited to physician-related factors, and excludes patient, system, and policy factors, as well as their interdependencies. Furthermore, we have yet to ascertain the views of ethnic minority patients on the contributors to diagnostic errors, specifically communication failures within the diagnostic process.

Methods: In 2019, the authors performed four focus groups of adult Latino community members in Spanish and English, in San Antonio, Texas. Participants were recruited through community centers, churches, and community health worker, or promotors, networks. Each participant experienced a primary care diagnostic workup in the past year. The focus groups, which were audio recorded and transcribed verbatim, discussed participant's perceptions of the communication (e.g., face-to-face, electronic, telephone, and written) with their primary care clinic team during a new symptom evaluation. The Spanish transcripts were translated into English by a professional translation service. Two reviewers coded the transcripts using the National Academy of Medicine's Diagnostic Process as a guiding framework. The major themes arose from inductive analysis of the data.

Results: Twenty-eight participants were included in focus groups. The average participant age was 54. Participants identified eight key contributors to communication-based errors in primary care. Language discordance, patient-physician mistrust, and objectification were the most commonly referenced contributing factors to communication failures during diagnostic workups. Participants acknowledged their position in a complex network of interdependent, communication-driven relationships, which serve to further alienate those unable to navigate the healthcare system.

Conclusion: Despite patient reporting communication failures, they are yet unmeasured by current methodologic advancements in the field of diagnostic safety. Further investigation into these processes may uncover roles for medical education, policy and health care organization interventions to improve patient safety.

\section{Diagnostic Impact of a Device-Enabled Remote “Tele-Dizzy” Consultation Service}

D. Gold, S. Peterson, A. McClenney, R. Tourkevich, A. Brune, W. Choi, A. Shemesh, B. Maliszewski, J. Bosley, J. Otero-Millan, M. Fanai, D. Roberts, N. Tevzadze, D. Zee, D. Newman-Toker

Johns Hopkins Medicine, Baltimore, MD

Problem: Missed stroke in the emergency department (ED) is a leading cause of misdiagnosis-related harms. The symptom most tightly linked to missed stroke is dizziness/vertigo, because these strokes mimic inner ear vestibular disorders closely. Diagnostic errors in acute dizziness/ vertigo are frequent for both vestibular disorders ( $\sim 80 \%$ misdiagnosed) and stroke $(\sim 35 \%$ missed $)$.

Description of the program: This is a case study of a hospital-level quality improvement intervention. We deployed a remote, deviceenabled (using video-oculography) Tele-Dizzy consultation service to improve diagnosis of dizziness and vertigo in the ED. Last year, we described the program. This year, we describe the results of this intervention.

Findings to date: Since July 1, 2017, we have offered a Tele-Dizzy consultation service during business hours. We have performed 214 consults at Johns Hopkins Hospital (JHH). We have seen improvements in diagnostic accuracy and reductions in unnecessary test utilization.

Accuracy: Without Tele-Dizzy, the non-specific R42 “dizziness” ICD10 code diagnose was made in 40\% of all patients with dizziness/vertigo in 2015 and 2018. Among those receiving a consultation, only 19.6\% ( $n=42 / 214)$ were given a non-specific code (i.e., the number of symptomonly non-diagnoses was cut in half among Tele-Dizzy patients). In addition, $>50 \%$ were given specific vestibular diagnoses ( $\mathrm{n}=116 / 214)$.

Utilization: Without Tele-Dizzy, the JH CT rate for all patients with dizziness/vertigo in 2015 and 2018 was 39\%. Among those receiving a consultation, only $2.3 \%(\mathrm{n}=5 / 214)$ were recommended to receive a CT scan (i.e., the rate of CT scans was reduced by more than $90 \%$ among Tele-Dizzy patients). Without Tele-Dizzy, the JH MRI rate for patients with dizziness or vertigo in 2015 and 2018 was $20 \%$. Among those receiving a consultation, 20\% $(n=43 / 214)$ were recommended to receive a MRI and/or MR angiogram (i.e., the rate of CTs was not reduced by simply ordering more MRI scans). Note that true reductions are likely larger since patients referred for Tele-Dizzy consultation are more likely than average to have undergone CT/MRI in usual care (because $\sim 50 \%$ of ED dizzy patients have obvious medical causes and typically undergo neither neuroimaging nor Tele-Dizzy consultation). 
Lessons learned: Our Tele-Dizzy service has provided greater diagnostic accuracy and, simultaneously, lower utilization for patients who undergo consultation. Those who undergo neuroimaging receive the correct test (MRI), rather than an incorrect one (CT). Our next steps will be to expand our services to two community hospitals and test the hypothesis of lower risks of missed stroke, using a larger sample.

\section{Tele-Dizzy Work Flow}
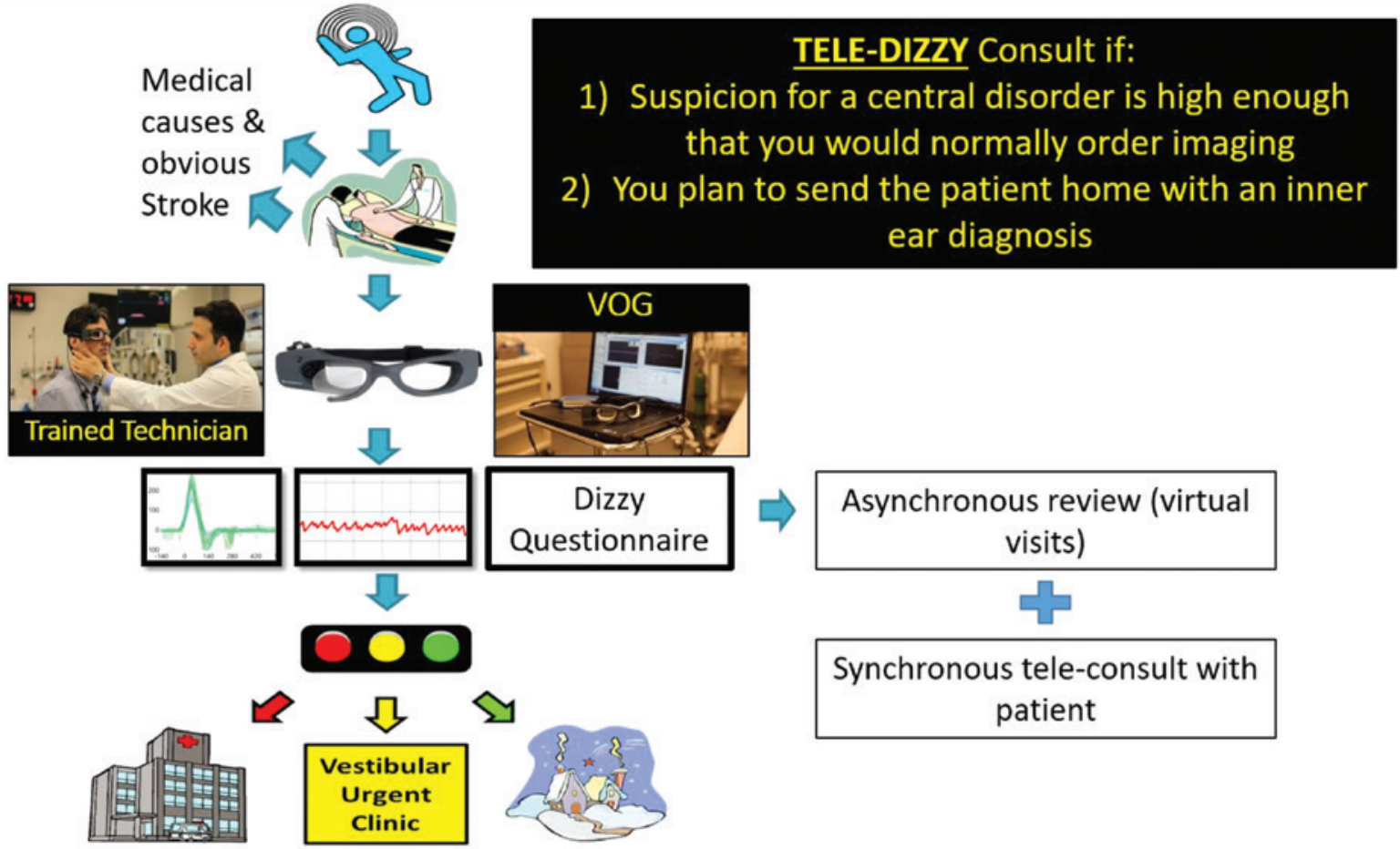

\section{Real-World Virtual Patients: Case-Based Simulation Using Actual History and Physical Exam Data to Improve Diagnosis}

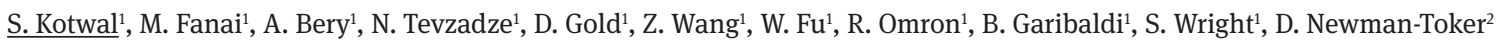

${ }^{1}$ Johns Hopkins Bayview Medical Center, Johns Hopkins University School of Medicine, Baltimore, MD

${ }^{2}$ Armstrong Institute, Center for Diagnostic Quality, Johns Hopkins University School of Medicine, Baltimore, MD

Purpose/Problem: The National Academy of Medicine recommends simulation training (typical and atypical cases) to improve diagnostic performance. Dizziness is a common symptom associated with significant financial burden and a high rate of misdiagnosis. We developed an interactive, virtual patient-based curriculum to improve the diagnostic approach to dizziness.

Study Description: Virtual patients (SIDD - Simulation-based curriculum to Improve Dizziness Diagnosis) were created in an interactive, screen-based microsimulation software environment. The software simulates a real bedside encounter, leveraging gaming approaches to incentivize a "least-moves" strategy to diagnosis with each item having associated virtual costs. Structured history and videotaped physical exams from an NIH-sponsored clinical trial of patients with acute dizziness were incorporated into each case simulation for maximal fidelity. We used a quasi-experimental pre-test/post-test nonequivalent comparison group study design. Both intervention (interns) and control groups (senior medical residents) completed 6 pretest virtual cases. Then, the interns were exposed to 9 hours of curricular material: online content plus SIDD (14 training cases). The residents were exposed to online content alone. Both groups were assigned 12 posttest virtual cases. Asymmetric intervention groups allowed us to compare SIDD to the cumulative clinical educational experience that trainees are exposed to for dizziness.

Study Assessment: Our primary outcome was posttest score, reflecting diagnostic accuracy (intervention vs. control). Secondary outcomes were change in accuracy scores, and cost assessment of the diagnostic testing ordered. Comparisons were made using Wilcoxon signed-rank tests. 
Outcomes: A total of 40 learners (22 interns and 18 residents) volunteered for the study. Residents completed 70 pretest and 150 post-test cases, while interns went through 125 pretest and 195 post-test cases. Table compares accuracy scores and costs of diagnostic workup for both the intervention and control groups during the pre and post-test periods, and also evaluates intra-group changes across the 2 time-periods.

Discussion: SIDD was able to significantly improve the diagnostic approach of novice clinicians (interns) such that they were more accurate and chose tests more wisely than residents in caring for those with acute dizziness.

Significance of findings: Conventional diagnostic teaching around complex presentations, like dizziness, do not work well. Exposing learners to multiple cases concurrently with specific diagnostic features and clues, allows for comparisons of similarities and differences that facilitate an appreciation of the subtleties of diagnostic reasoning. Future plans are to apply this condensed educational experience across a broad range of common symptoms that are costly and associated with high rates of misdiagnosis.

Table: Summary of Test Results

\begin{tabular}{|c|c|c|c|c|c|c|c|c|c|}
\hline & \multicolumn{3}{|r|}{ Pre Test } & \multicolumn{3}{|c|}{ Post Test } & \multicolumn{3}{|r|}{ Change } \\
\hline & $\begin{array}{l}\text { Control } \\
(n=16)\end{array}$ & $\begin{array}{r}\text { Intervention } \\
(n=22)\end{array}$ & $p$ value & $\begin{array}{l}\text { Control } \\
(n=17)\end{array}$ & $\begin{array}{r}\text { Intervention } \\
(n=18)\end{array}$ & $p$ value & $\begin{array}{l}\text { Control } \\
(n=15)\end{array}$ & $\begin{array}{r}\text { Intervention } \\
(n=18)\end{array}$ & $p$ value \\
\hline \multicolumn{10}{|c|}{ Test Score } \\
\hline mean & 34.4 & 36 & 0.61 & 31.8 & 53.1 & 0.001 & -3.6 & 18.1 & 0.02 \\
\hline Range & $0-100$ & $0-83.3$ & & $11.1-100$ & $25-90$ & & $-88.9-100$ & $-25-53.3$ & \\
\hline \multicolumn{10}{|l|}{ Cost (\$) } \\
\hline mean & 983.3 & 955.4 & 0.98 & 1235.90 & 736.30 & $<0.01$ & 236.1 & -197.9 & 0.009 \\
\hline range & $355.8-1639$ & $456.5-2144$ & & $504.3-2154.7$ & $335.5-1125.5$ & & $-639.7-966.2$ & $-1446.1-379.2$ & \\
\hline
\end{tabular}

Wilcoxon tests were used

\section{Measuring and Improving the Accuracy of Sepsis Diagnosis Using Simulated Patients Leads to Patient-Level Improvements in Four Healthcare Systems}

L. Peabody $^{1}$, D. Paculdo², T. Burgon², E. De Belen², D. Tamondong-Lachica ${ }^{2}$

${ }^{1}$ QURE Healthcare, University of California San Francisco, University of California Los Angeles, CA

${ }^{2}$ QURE Healthcare, San Francisco, CA

Background: Sepsis is one of the leading causes of death in U.S. hospitals and one of the most expensive. Sepsis can be misdiagnosed or recognized late. These diagnostic errors lead to escalating complications, costs and mortality. Notwithstanding the clinical urgency, few studies have evaluated the prevalence of sepsis misdiagnosis. Fewer still have investigated if the problem of misdiagnosis can be improved.

Methods: Using a validated, patient simulation-based practice measurement tool, Clinical Performance and Value? vignettes, we quantified diagnostic accuracy among practicing hospitalists. We then determined whether personalized and group feedback on evidence-based care decisions on the simulated care led to improvements in real-world patient quality measures.

Between March 2016 through March 2019, 560 hospitalists from four large healthcare systems across the United States cared for simulated patients presenting with symptoms indicative of sepsis. With each case, we measured and scored provider diagnostic accuracy. Providers received their diagnostic scores along with individual and group-level feedback on how to improve their workup, diagnosis and treatment. Each healthcare system participated in four to six serial rounds of measurement, benchmarking, and feedback.

Results: At baseline, hospitalist providers recognized sepsis in only 59.3\% of their patients. This increased to $75.2 \%$ by the last round $(\mathrm{p}<0.001)$. As diagnostic accuracy improved, we saw a 22.1 percentage point increase in adherence to all elements of the three-hour sepsis bundle $(\mathrm{p}<0.001)$, including a 9.6 percentage point increase in ordering correct antibiotic regimens $(\mathrm{p}=0.004)$ (see table). We concurrently collected real-world outcomes data in a pre-post analysis. We found reductions of up to $18 \%$ in observed/expected length-of-stay and riskadjusted cost reductions up to $20 \%$, without any increase in complications or mortality across all four sites.

Conclusion: At baseline, hospitalists misdiagnosed sepsis in over $40 \%$ of the interactive simulated patients. After multiple rounds of measurement and feedback, diagnostic errors decreased significantly and care improved markedly. Using available real-world data, we found corresponding improvements in actual patients suggesting that better diagnostic accuracy led to better patient care and lower costs. 


\begin{tabular}{lrrr}
\hline & Baseline & Last Round* & p-value \\
\hline Sepsis diagnosis & $59.3 \%$ & $75.2 \%$ & $<0.001$ \\
Sepsis bundle & $30.2 \%$ & $52.3 \%$ & $<0.001$ \\
Serum lactate & $51.0 \%$ & $72.9 \%$ & $<0.001$ \\
Blood culture & $71.3 \%$ & $82.2 \%$ & $<0.001$ \\
Hydration & $68.5 \%$ & $80.9 \%$ & $<0.001$ \\
Antibiotics (any) & $93.9 \%$ & $99.8 \%$ & $<0.001$ \\
Correct antibiotics & $46.2 \%$ & $55.8 \%$ & 0.004 \\
Correct admission & $64.3 \%$ & $67.7 \%$ & 0.295 \\
\hline
\end{tabular}

*Either Round 4 or 6 , depending on healthcare system.

\section{Implementation of e-Triggers for Measurement of Diagnostic Errors in a Large Health Care System}

$\underline{\text { H. Singh }}{ }^{1}$, D. Upadhyay ${ }^{2}$, S. Korukonda ${ }^{2}$ J. Hunt ${ }^{2}$, D. Torretti ${ }^{3}$

${ }^{1}$ Center for Innovations in Quality, Effectiveness and Safety, Michael E. DeBakey Veterans Affairs Medical Center and Baylor College of Medicine, Houston, TX

${ }^{2}$ Safer Dx Learning Lab, Division of Quality and Safety, Geisinger, Danville, PA

${ }^{3}$ Geisinger Medical Center, Geisinger, Danville, PA

Background: Health care organizations (HCOs) should use their ever-increasing stores of electronic data for learning, research, and quality improvement. Electronic trigger (e-trigger) tools, which mine vast amounts of clinical and administrative data to identify signals for likely adverse events, offer a promising method to do so. We used a knowledge discovery framework, the Safer Dx Trigger Tools Framework, to develop and implement e-trigger tools to measure diagnostic errors using comprehensive longitudinal electronic health record (EHR) data in a large integrated care-delivery system.

Methods: Building on prior work in primary care, we developed and refined two rules-based e-trigger algorithms to identify patterns of care suggestive of missed or delayed diagnoses: 1) a primary care (PC) visit followed by unexpected hospitalization within 14 days and 2) emergency department (ED) discharge followed by unexpected hospitalization within 14 days. To increase the algorithm's positive predictive value (PPV), we excluded patients with terminal or metastatic cancers, hospice, palliative care or long-term care encounters in the last 12 months, $>85$ years, and admissions resulting from an accident or trauma or to psychiatric floor. The e-trigger algorithm was tested and then applied in a large EHR data warehouse. We recruited practicing physicians as reviewers and trained them to review trigger-positive records for diagnostic errors, defined as missed opportunities in diagnosis (MODs). Blinded-reviewers independently determined presence/absence of MODs in randomly selected $\sim 100 \mathrm{PC}$ and $\sim 100$ ED triggered visits using the Revised Safer Dx instrument. We analyzed MODs to identify diagnostic process breakdowns and contributory factors.

Results: The e-trigger queried > 690,000 PC and 47,000 ED visits during 2017 and yielded 130 and 354 trigger-positive visits respectively. Upon review, we found MODs in 16 of $102 \mathrm{PC}$ visits (PPV =15.68\%) and 28 of $105 \mathrm{ED}$ visits (PPV=26.67\%). While heart failure exacerbation emerged as most common missed diagnosis in PC, ED cases were more heterogenous and no common target emerged. Most common symptoms associated with missed diagnoses were abdominal pain and shortness of breath in PC and abdominal pain and fever in ED. The most common breakdown involved patient-provider encounter in both settings, including problems with adequate history elicitation, physical examination, ordering appropriate diagnostic tests for further work-up, and with data integration and interpretation.

Conclusions: Measurement using e-triggers substantially reduces the number of records HCOs need to review to those at highest risk of preventable diagnostic harm. Routine implementation could generate organizational learning and lead to personalized feedback for diagnostic safety improvement.

\section{The Committee to Improve Clinical Diagnosis - An Organization's First Approach to Address Diagnostic Safety}

$\underline{\text { D. Upadhyay }}^{1}$, D. Torretti ${ }^{1}$, C. Schuerch ${ }^{1}$, Y. Mehta ${ }^{1}$, B. Hohmuth ${ }^{1}$, P. Lamphear ${ }^{1}$, H. Singh ${ }^{2}$

${ }^{1}$ Geisinger Medical Center, Geisinger, Danville, PA

${ }^{2}$ Center for Innovations in Quality, Effectiveness and Safety, Michael E. DeBakey Veterans Affairs Medical Center and Baylor College of Medicine, Houston, TX

Statement of problem: Very few health care organizations have created an infrastructure to stimulate processes to identify and learn from diagnostic errors. 
Description of the program: In 2017, a large integrated health care organization (HCO) created 'The Committee to Improve Clinical Diagnosis (CICD)' as a first step to develop a learning health system focused on diagnostic safety. The CICD evolved from a workgroup organized in 2014 into a multi-disciplinary committee whose membership includes department/quality directors from different clinical disciplines: internal, hospital, community and emergency medicine, surgery, pediatrics, woman's health, oncology, radiology and laboratory medicine, and representatives from quality and safety, medical informatics, patient experience, IT and education divisions. The CICD created a formal charter with primary functions that include:

- $\quad$ development of innovative approaches to identify and address missed opportunities in diagnosis (MODs)

- implementation of a feedback process

- $\quad$ categorization of MODs and monitoring of patterns

- $\quad$ recommendations to address high volume or high-risk cases.

The CICD reviews cases from various sources for potential MODs and seeks to identify specific learning points in each case. Once reviewed, the cases are shared with relevant department directors who provide feedback in a constructive manner, seek input from those involved and share any lessons learned broadly. A toolkit on providing feedback within this setting has been developed and its utility is being assessed.

Findings: The CICD's work and impact has continued to increase. In the 267 cases reported to the CICD thus far, MODs have been identified in 152 cases, most commonly from the ED. Based upon the data, CICD has facilitated implementation of performance improvement opportunities such as improving pediatric sepsis care, closing the loop on incidental findings (e. g. pulmonary nodules) and communication of imaging results.

Lessons learned: At the organization level, a supportive and engaged departmental leadership was critical to CICD development and growth. Data gathering, analysis and feedback mechanisms within departments and across the institution are being refined to further develop and strengthen a learning health system for diagnosis.

Other HCOs should consider creating a similar committee or advisory council to stimulate exploration of diagnostic events. Such committees may partner with researchers to develop innovative evidence-based approaches to identify and analyze MODs. Initial activities could include developing the institutional framework to gather intelligence related to diagnostic events. Such activities can constructively influence and promote a culture that values diagnostic excellence and transparency.

\title{
Advancing Diagnostic Safety Research: Results of a Systematic Research Priority Setting Exercise
}

\author{
$\underline{\text { L. Zwaan }}^{1}$, R. El-Kareh², A. Meyer ${ }^{3}$, H. Singh ${ }^{3}$
}

${ }^{1}$ Erasmus University Medical Center Rotterdam, Institute of Medical Education Research Rotterdam, Netherlands

${ }^{2}$ University of California at San Diego, Dept of Medicine, La Jolla, CA

${ }^{3}$ Center for Innovations in Quality, Effectiveness and Safety, Michael E. DeBakey VA Medical Center and Baylor College of Medicine, Houston, TX

Background: While the number of research projects on diagnostic safety are increasing, much of this research is fragmented with little assurance it focuses on the right questions. Additional insights are needed on the most urgent and impactful questions in order to promote research that is more actionable. We conducted a systematic research priority-setting exercise to identify and prioritize research questions to advance the field of diagnostic safety.

Methods: We used a combination of established systematic research prioritization methods including those from the Child Health and Nutrition Research Initiative (CHNRI) and an in-person expert meeting. We first identified a diverse group of expert researchers in diagnosis and related disciplines based on their scholarly output. Using a structured questionnaire, we collected (in free-text) up to five research questions they considered the most important to move the field forward in the next 3-5 years. To stimulate their best questions, we asked them to consider five prioritization criteria: (1) usefulness, (2) answerability, (3) effectiveness, (4) potential for translation and (5) maximal potential for effect on diagnostic safety. The project team reviewed all submitted questions for appropriateness while removing duplicates. This list was then prioritized at an in-person 1.5-day expert meeting in April 2019 to select high-priority questions. As a final prioritization step, which is ongoing, a weighted priority score will be calculated for each of the high-priority questions by using data from a) researchers scoring all high-priority questions using the five prioritization criteria, and b) weights for each prioritization criterion determined by a diverse group of diagnostic safety stakeholders who responded to a structured questionnaire.

Results: Of 207 invited researchers, 78 submitted 333 questions that addressed burden, contributory factors and interventions for diagnostic error. Expert meeting participants $(n=21)$ discussed 177 consolidated questions and prioritized the list to 50. Of 67 invited stakeholders, 35 submitted weights for the prioritization criteria: thus far the criterion 'maximal potential for effect on diagnostic safety' has received the highest weight. All 50 high-priority questions will be sent out to the 78 researchers in July 2019 for scoring. Using these data and the weights from stakeholders, we will determine the final prioritization score for each question to identify 20 questions representing the highest research priority (final list expected September 2019).

Conclusion: We systematically identified the top research priorities for advancing diagnostic safety using transparent and objective methods that included input from a large group of researchers and stakeholders. 


\title{
Oral Abstracts
}

\section{Characterization of Diagnostic Error in Provider-Referred Cases of Hospitalized Patients}

\author{
P. Barish, D. Sterken, K. Raffel, M. Kantor, C. Fenton, A. Lai, S. Ranji \\ University of California San Francisco, San Francisco, CA
}

Background: Diagnostic errors are common in hospitalized patients, but the drivers of error and clinical impact are not well-understood. We performed a structured review of cases referred to a quality improvement committee in Hospital Medicine to better understand diagnostic error in the acute-care setting.

Methods: Our study included Hospital Medicine cases referred to an internal Case Review Committee from March 2015 to July 2017 by both physician and non-physician providers. After an initial in-depth physician review, cases containing possible diagnostic errors were selected for further analysis. A structured review of the electronic medical record was completed independently by two trained board-certified internists, supported by consensus review in discrepant cases. Diagnostic errors were identified using the "Safer Dx Instrument," a validated tool to identify diagnostic error in primary care. When identified, errors were then characterized using the Diagnostic Error Evaluation and Research (DEER) taxonomy tool, an error classification system.

Results: 80 provider-referred cases to the committee were reviewed. A total of 22 (27.5\%) contained a diagnostic error. In 13 of the 22 cases (59\%) the error was determined to have a "major" impact (death or permeant disability) and in 6 cases (27\%) the error was judged as having "moderate" impact (short-term morbidity, increased length of stay, or invasive procedure). 11 of these cases (50\%) were in patients admitted to the Intensive Care Unit (ICU). Errors represented a breadth of diagnoses and no diagnostic category (i.e. sepsis) was highly represented in this sample. In the DEER taxonomy analysis the most common lesions in the diagnostic process occurred in Assessment, Tests, and History. The most common subcategories contributing to error were failure/delay in considering correct diagnosis [14, 64\%], failure/delay in ordering needed tests [13,59\%], failure to recognize/weight urgency [11, 50\%] and suboptimal weighing of a critical piece of history [11, 50\%].

Conclusions: Provider case referral is an effective method to identify diagnostic error in hospitalized patients. The prevalence of diagnostic error among our cohort was $27.5 \%$, significantly higher than estimates for the general inpatient population, and errors had a more serious clinical impact than previous estimates. The clinical acuity of the population was also high, with half of the patients admitted to the ICU. In line with previous work categorizing diagnostic error, lesions in cognition appear to be the predominant drivers of error in this population, highlighting the importance of efforts to improve provider diagnostic reasoning.

\section{Design and Validity Evidence for an Illness Script Scoring Rubric}

$\underline{\text { P. Bergl }}{ }^{1}$, S. Dunbar ${ }^{1}$, M. Plesac ${ }^{2}$, A. Olson ${ }^{2}$, J. Patel ${ }^{1}$

${ }^{1}$ Medical College of Wisconsin, Milwaukee, WI

${ }^{2}$ University of Minnesota, Minneapolis, MN

Purpose: Illness scripts (IS) are compiled mental representations of disease stored in clinicians' memories, and experts have richer IS than novices. Existing tools to assess IS, such as the script concordance test, compare how learners activate IS relative to experts but cannot clarify mechanisms leading to concordance (or discordance). Further, such tools may not reliably replicate real clinical experiences. Alternative assessment tools which deconstruct and measure IS components would allow educators to gauge learners' IS formation, thereby allowing for targeted feedback. Longitudinal IS assessment also would aid in tracking learners' development in diagnostic reasoning. The primary purpose of this study was to iteratively develop and validate an IS scoring tool to assess learners' written IS quality.

Description of study: As part of a diagnostic reasoning curriculum, internal medicine residents were prompted to write out IS by describing prototypical presentations of cardiac and pulmonary diseases tested on the American Board of Internal Medicine certification examination. We developed a rubric to score these written IS by examining other written reasoning assessment tools in the literature; reviewing trainees' responses as a team and formulating assessment options based on IS components; and pilot-testing with two lead investigators. After pilot testing, four assessors independently reviewed and scored trainees' written IS. The final rubric (Figure 1) included two scores of IS quality: (1.) total score based on the sum of the individual rubric components and (2.) overall gestalt assessment.

Outcomes: Eighty-seven (87) trainees wrote IS for four different diseases, yielding 348 IS for review. Two raters reviewed all 348 IS and strongly agreed on total score summed from the rubric (Spearman's rho $=0.643, p<0.0001$ ) and overall gestalt assessment of IS quality (Spearman's rho $=0.683, \mathrm{p}<0.0001$ ). Subsequently, two additional raters ( 4 total) independently reviewed subsets of IS written by first-year residents ( $\mathrm{n}=98$ scripts). Among all 4 raters, intra-class correlation coefficients (ICC) were extremely robust for total score summed from the rubric (ICC $=0.943, \mathrm{p}<0.0001$ ) and overall gestalt assessment $($ ICC $=0.922, \mathrm{p}<0.0001$ ).

Discussion: We found strong agreement among educators' assessments of written IS using both a scored rubric and overall gestalt. This study provides initial validity evidence for a rubric to score written IS within internal medicine. Limitations include the narrow scope of diseases tested and the constrained range of subjects' expertise. Further testing and rubric validation is underway across multiple institutions. 


\begin{tabular}{|c|c|c|}
\hline Component & Definition & Scoring \\
\hline $\begin{array}{l}\text { Epidemiologic } \\
\text { factors }\end{array}$ & $\begin{array}{l}\text { A combination of patient factors } \\
\text { (age, sex, habits, occupation, family } \\
\text { history) and contextual factors (time } \\
\text { of year, care setting) that influence the } \\
\text { probability of the disease. }\end{array}$ & $\begin{array}{l}0=\text { None listed } \\
1=\text { Minimally considered } \\
2=\text { Somewhat considered } \\
3=\text { Strongly considered }\end{array}$ \\
\hline Pathophysiology & $\begin{array}{l}\text { Any anatomic, biologic, physiologic, } \\
\text { or biochemical insult or lesion that } \\
\text { contributes to the development of the } \\
\text { disease. }\end{array}$ & $\begin{array}{l}0=\text { Not listed } \\
1=\text { Minimally considered } \\
2=\text { Somewhat considered } \\
3=\text { Strongly considered }\end{array}$ \\
\hline $\begin{array}{l}\text { Clinical signs and } \\
\text { symptoms }\end{array}$ & $\begin{array}{l}\text { Patient complaints, physical } \\
\text { examination findings, laboratory data, } \\
\text { typical imaging findings, or other } \\
\text { diagnostic data that supports the } \\
\text { diagnosis. }\end{array}$ & $\begin{aligned} 0= & \text { Not listed } \\
1= & \text { Many common or typical findings are missing from script. } \\
2= & \text { Most common or typical findings are listed, but not all domains } \\
& \quad \text { (symptoms, signs, diagnostic test results) are discussed. } \\
3= & \text { Script is a complete/comprehensive list of typical findings across } \\
& \text { most domains (symptoms, signs, diagnostic test results). }\end{aligned}$ \\
\hline Accuracy & $\begin{array}{l}\text { Script elements reflect common } \\
\text { knowledge on the disease and how it } \\
\text { presents. }\end{array}$ & $\begin{array}{l}0=\text { Mostly inaccurate } \\
1=\text { Somewhat inaccurate } \\
2=\text { Somewhat accurate } \\
3=\text { Very accurate }\end{array}$ \\
\hline $\begin{array}{l}\text { Clinical } \\
\text { relevance and } \\
\text { discrimination }\end{array}$ & $\begin{array}{l}\text { Script generally helps discriminate } \\
\text { this condition, activates expert's own } \\
\text { script, and/or helps exclude alternative } \\
\text { diagnoses that present similarly. }\end{array}$ & $\begin{array}{l}0=\text { Not helpful at all (i.e. does not make expert think of diagnosis at all) } \\
1=\text { Not very helpful } \\
2=\text { Somewhat helpful } \\
3=\text { Very helpful (i.e. immediately brings diagnosis to forefront of } \\
\quad \text { expert's mind) }\end{array}$ \\
\hline
\end{tabular}

\section{Overall Rating of Script}

Highly underdeveloped, incomplete, and/or incorrect

understanding of how this disease presents $\begin{array}{lllllll}1 & 2 & 3 & 4 & 5 & 6 & 7\end{array}$

(lowest $\rightarrow \rightarrow \rightarrow \rightarrow \rightarrow \rightarrow$ highest)

Perfect, comprehensive description

of how this disease presents

\section{A Pilot of a Shared Decision-Making Tool to Improve Diagnostic Plan Communication in Two Unique Internal Medicine Practices}

L.P. Brito, I. Hargraves, A. Majka, J. Matulis, K. Shaw

Mayo Clinic, Rochester, MN

Statement of problem: Diagnostic errors lead to inappropriate patient care, poor outcomes or increased cost. Face-to-face interaction between patients and clinicians is a focal point in the diagnostic process as this is where development and communication of the initial diagnostic plan most commonly occurs. Unless these diagnostic plans are communicated effectively it is likely that the risk of diagnostic error will increase. Additionally, poor interaction can lead to diagnostic plans that are impractical for the patient to implement, fully understand or not pertinent. Intervention: Shared decision making is a patient centered process where patients and clinicians communicate with the goal of developing a shared understanding of the patient's circumstances, values and preferences and formulate a plan which makes sense intellectually, practically, and emotionally. As such shared decision making has the potential to reduce diagnostic error by facilitating diagnostic plan development. To date, shared decision-making interventions have not been developed for the purpose of facilitating effective diagnostic communication. We are developing a shared decision-making intervention to support diagnostic conversations and plan formation.

Methods: We formed a multidisciplinary team of clinicians, designers, and shared decision-making experts. Discussions within the group led to an initial conceptualization of diagnostic error arising through patient- clinician interaction. This theoretical work was accompanied by empirical observation of Diagnostic conversations within real clinical practice. Using a human centered design approach the designers observed or video recorded 23 patient visits within a primary care internal medicine practice and a referral internal medicine practice caring for executive, development, and International patient's. They then reviewed these videos and observation notes and identified themes. Following this learning, prototype conversation aids were developed and iteratively refined through observing their use within these encounters. Themes identified: Diagnosis formation and decision-making often arise from multiple situations. Treatment focused shared decision-making approaches may not be appropriate. Patients often have limited ability to contribute to diagnosis formation. Patient's are sometimes confused about diagnostic next steps, what they require, and why they are being done. 
Next steps: Development and evaluation of prototype diagnostic conversation aides focused on:

- Clarifying the situation out of which diagnostic activities arrive.

- Introducing brief pauses to confirm patient understanding.

Supporting shared decision-making for diagnostic plans based on downstream consequences, patient burden, and end goals of this diagnostic plan.

\title{
Harnessing Patients' and Families’ Experiences to Identify Diagnostic Errors
}

$\underline{\text { T. Giardina }}^{1}$, U. Shahid ${ }^{1}$, S. Korukonda ${ }^{2}$, V. Vaghani ${ }^{1}$, D. Upadhyay ${ }^{2}$, H. Singh ${ }^{1}$

${ }^{1}$ Michael E. DeBakey Veterans Affairs and Baylor College of Medicine, Houston, TX

${ }^{2}$ Geisinger, Danville, PA

Background: Patients can identify safety issues not captured by traditional safety measurement methods and unsurprisingly, interest in using patients' experiences to inform quality and safety improvements is growing. While prior studies have focused on documenting the frequency, demographics, and categories of patient complaints, to our knowledge, none have analyzed them specifically to identify diagnostic errors. We evaluated the use of patient complaints as a strategy to identify diagnostic errors.

Methods: We reviewed patient complaints submitted to a large health care organization (HCO) from August to December 2017. To avoid service and satisfaction specific issues, we selected complaints based on how the HCO categorized and stored them in the data collection system (e.g., accident/injury, attitude/behavior of clinicians/staff, clinical care issues, delay in care/test results/discharge, and discharge issues). Two investigators reviewed all one sentence complaint summaries and identified cases as 'potentially concerning for diagnostic error' using the National Academies definition. For all potential cases, a clinical reviewer evaluated the associated investigation report and the patient's medical record comprehensively. This was, followed by team discussion of cases to confirm presence/absence of diagnostic errors.

Results: We reviewed 1865 patient complaint summaries and identified 177 (9.5\%) potentially concerning reports. Sample was majority female $(69.2 \%)$ and white (100\%), with a mean age of 44 (SD 28.2, Range: 9months-91years). Upon full cohort review and analysis, we identified 39 diagnostic errors (2.1\%). Most were categorized as "Clinical Care - provider" (27,69.2\%), defined as concerns/questions related to the inpatient or outpatient care provided by providers. Although the diagnoses were diverse, the most common were cancer, missed fracture, and Lyme disease. Upon qualitative analysis, we found that patients and families indicated they were unhappy with the quality of clinical care they received. In certain cases, patients felt that the encounter was unhelpful and that their doctor was dismissive or did not listen. This included failure to order testing or incorrect and incomplete reading of radiology results.

Conclusion: Analysis of patient complaints reveals that patients and families reported failures in the diagnostic process, many occurring during the care provided at patient-clinician encounter. This source of data could be potentially harnessed for identifying diagnostic errors from the patient perspective - enabling their future use as uniquely patient-centered quality assurance tools. Systematically incorporating patients' perspectives into routine organizational activities and clinical operations may lead to meaningful improvements in the patient's diagnostic experience.

\section{Diagnostic Knowledge and 90-day Risk for All-cause Mortality, Emergency Department Visits, and Emergency Hospitalizations for Conditions at Risk for Diagnostic Errors After Outpatient Visits}

\author{
$\underline{\text { B. Gray }}^{1}$, J. Vandergrift ${ }^{1}$, R. McCoy ${ }^{2}$, R. Lipner ${ }^{1}$, B. Landon ${ }^{3}$ \\ ${ }^{1}$ American Board of Internal Medicine, Philadelphia, PA \\ ${ }^{2}$ Division of Community Internal Medicine, Department of Medicine, Mayo Clinic, Scottsdale, AZ \\ ${ }^{3}$ Harvard Medical School and Beth Israel Deaconess Medical Center, Boston, MA
}

Background: Diagnostic error is identified as a key health care delivery concern with large impacts on morbidity and mortality. Yet no study has quantified associations between adverse outcomes that might result from diagnostic errors and one potentially large contributor to these errors, deficiencies in diagnostic knowledge.

Method: We studied 1,409 mid-career general internists (initially certified in 2000) and the fee-for-service Medicare patients they cared for during the year after, 2009 to 2011, they took their American Board of Internal Medicine's (ABIM) Internal Medicine Maintenance of Certification (IM-MOC) exam. These patients presented for 48,071 outpatient visits for potentially new problems (i.e., no prior outpatient or inpatient visit for a 90-day period) with symptoms at risk for 13 acute previously identified diagnostic error-prone conditions. We estimated associations between diagnostic knowledge as measured by percent correct diagnosis related IM-MOC exam questions and risk of an emergency department (ED) visit, emergency hospitalization (hospitalizations initiated through the ED) and all-cause mortality within 90 days of these index visits. For hospitalizations and ED visits we considered first occurrences where the index visit diagnosis was related to the applicable 
diagnostic error-prone condition outcome (e.g., dizziness for stroke). We estimated associations using a Probit model that accounted for index visits being nested within physicians, and controlled for overall exam performance, physician and practice characteristics, and a variety of patient risk factors.

Results: Ninety days after index visits $0.7 \%$ of patients died, $1.1 \%$ and $1.4 \%$ of patients had a subsequent emergency hospitalization and ED visit respectively for a related diagnostic error-prone condition. Treatment by an internist scoring in the top versus bottom third of diagnostic knowledge was associated with significant risk reductions for all adverse outcomes: 38.9\% for all-cause mortality $(\mathrm{P}=0.003)$, 29.0\% for emergency hospitalization $(\mathrm{P}=0.001)$ and $28.8 \%$ for an ED visit $(\mathrm{P}=0.005)$.

Supporting the validity of our approach, falsification sensitivities demonstrated that these associations were statistically insignificant $(\mathrm{P}>0.50)$ and less than one tenth as large in magnitude when the sample was limited to index visits without diagnoses related to the diagnostic error-prone conditions. Similarly, elective hospitalizations, which were related to the overall level of hospitalizations, were not associated with diagnostic knowledge $(\mathrm{P}=0.51)$.

Conclusion: A measure of general diagnostic knowledge on an ABIM IM-MOC exam was associated with less adverse outcomes for patients presenting with conditions at risk for serious diagnostic errors.

\title{
The "Mechanics" of Teaching Clinical Reasoning to Year 1 Medical Students
}

\author{
$\underline{\text { H. Hoar }}$ \\ Baystate Medical Center, Springfield, MA
}

Purpose: Teachers of clinical reasoning have long believed the maxims that "clinical reasoning cannot be taught in the absence of medical knowledge" 1 and "clinical reasoning should be taught as early in training as possible." 2 These statements create an apparent paradox for those tasked with teaching the principles and practice of clinical reasoning to Year 1 medical students. We describe a novel method to draw on students' real-world experience to teach clinical reasoning principles at the very beginning of Year 1.

Description: When the University of Massachusetts Medical School opened a new branch campus at Baystate Medical Center, we implemented a new clinical reasoning curriculum within the existing Doctoring and Clinical Skills course (DCS). In the traditional DCS curriculum, the first session is dedicated to teaching students to obtain the 7 cardinal features of the HPI. In the new DCS curriculum, we used a novel approach to teach the HPI with a clinical reasoning focus. As Dhaliwal describes3 "the cognitive task of the mechanic is virtually identical to that of the physician: both use history, examination, and tests to diagnose and repair a complicated machine in the context of a human relationship.” This very first session of the DCS course was co-taught by a clinician and a local auto mechanic. While the mechanic tried to diagnose car problems in real time, students completed a worksheet (see attached).

Outcomes: The session was well-received and was even featured in a local news story about the new medical school campus. Student ratings and comments of the session and entire curriculum will be shared. Overall, the ratings and comments for the session were highly favorable. Discussion: The "Mechanics of Reasoning" curriculum is a novel and well-received method of teaching core clinical reasoning principles to first-year medical students. It can be effectively implemented right at the beginning of medical school and seems to foster genuine interest and enthusiasm for clinical reasoning.

Significance of findings: The use of real-world analogy in teaching clinical reasoning may be an effective approach to introduce clinical reasoning concepts to medical trainees early in their training.

\section{Improving Diagnostic Calibration by Providing Feedback to General-Practice Residents}

\author{
J. Kuhn ${ }^{1,2}$, S. Mamede², P. van den Berg' ${ }^{1}$ L. Zwaan², P. Bindels', T. van Gog ${ }^{3}$ \\ ${ }^{1}$ General Practice, Erasmus MC, Rotterdam, Netherlands, \\ 2iMERR, Institute of Medical Education Research Rotterdam, Erasmus MC, Rotterdam, Netherlands \\ ${ }^{3}$ Pedagogical and Educational Sciences, University Utrecht, Utrecht, Netherlands
}

Background: Physicians do not always estimate their diagnostic performance correctly, i.e. they show inaccurate diagnostic calibration. Especially overconfidence in one's diagnosis can be the reason for diagnostic errors and maltreatment (Berner and Graber 2008). One study successfully improved physicians' diagnostic calibration by providing them with feedback on their previous diagnostic performance (Nederhand, Tabbers et al. 2018). This study only included relatively easy cases. It has been found, however, that physicians have more trouble to calibrate correctly for difficult cases (Meyer, Payne et al. 2013). It is yet unknown whether feedback can also improve diagnostic calibration for difficult cases. Therefore, we conducted a study to investigate whether general-practice residents' diagnostic calibration improves when receiving feedback as they diagnose several difficult cases.

Methods: Participants were 69 residents in the 1st year of their general-practice training at the Erasmus Medical Center in Rotterdam. They were randomly assigned to either the feedback condition or the no-feedback condition. Under the feedback condition, participants (1) read a case, (2) gave the most likely diagnosis, (3) rated the confidence they had in their diagnosis, and (4) received the correct diagnosis, i.e. 
feedback for this case. They then moved on to the next case until 12 cases had been diagnosed. Under the no-feedback condition, participants followed the same procedure but without step 4, i.e. without receiving feedback for the cases.

Results: We are currently analyzing the data to see whether diagnostic calibration improves during the study and whether this differs between both conditions. The results will be available before the DEM conference.

Conclusion: This study will show whether a simple feedback intervention can help physicians to better estimate their diagnostic performance and to better monitor themselves. Emphasizing continuous feedback in physicians' training or even in the clinical workplace might help to improve diagnostic calibration and therefore prevent diagnostic errors due to overconfidence.

\title{
Pediatric Emergency Physicians' Perspectives on Diagnostic Safety - Results from an International Survey
}

\author{
$\underline{\text { P. Mahajan }}{ }^{1}$, J. Grubenhoff ${ }^{2}$ J. Cranford ${ }^{1}$, K. Shaw 3 , J. Chamberlain ${ }^{4}$, R. Ruddy ${ }^{5}$, A. Belle ${ }^{1}$, N. Kuppermann 6 , H. Singh ${ }^{7}$ \\ ${ }^{1}$ Department of Emergency Medicine, University of Michigan, Ann Arbor, MI \\ ${ }^{2}$ University of Colorado School of Medicine, Department of Pediatrics, Section of Emergency Medicine, Aurora, CO \\ ${ }^{3}$ Division of Pediatric Emergency Medicine, Department of Pediatrics, University of Pennsylvania, Children's Hospital of Philadelphia, \\ Philadelphia, PA \\ ${ }^{4}$ Division of Pediatric Emergency Medicine, Department of Pediatrics, Children's National Medical Center, Washington, D.C. \\ ${ }^{5}$ Department of Pediatrics, University of Cincinnati College of Medicine, Cincinnati Children's Hospital Medical Center, Cincinnati, OH \\ ${ }^{6}$ Departments of Emergency Medicine and Pediatrics, University of California-Davis School of Medicine, Sacramento, CA \\ ${ }^{7}$ Center for Innovations in Quality, Effectiveness and Safety, Michael E. DeBakey Veterans Affairs Medical Center, Baylor College of Medicine, \\ Houston, TX
}

Background: Knowledge about diagnostic safety and how to reduce missed opportunities for improving diagnosis (MOIDs) in pediatric emergency departments (EDs) is scant. We solicited ED physicians' insights into various determinants of diagnostic safety in the ED.

Methods: We developed a two-part, web-based survey and pilot tested it before administering it to physicians participating in the Pediatric Emergency Research Networks (PERN), a global research network of pediatric EDs. In Part 2 (described here) physicians provided perspectives related to enhancing the understanding of diagnostic safety in pediatric EDs.

Results: Of 1208 physicians, 267 (22.1\%) responded ( $\mathrm{M}$ age $=48$ years [SD =9.9], 56\% female, mean years in practice $=13$ years [SD =9.7]). Most physicians ranked MOIDs to be associated with medical presentations most commonly (87\%), followed by surgical diagnoses (10\%) and trauma (2\%). Over half (56\%) felt that MOIDs were more likely to occur in conditions that are frequently seen in the ED, while $44 \%$ felt they were more likely in uncommon conditions. Although there were a wide variety of conditions reported where MOIDs were likely, most (72\%) reported that acute abdominal pain, with a final diagnosis of appendicitis, was a condition commonly prone to MOIDs; other diagnoses associated with MOIDs included pneumonia (31\%), sepsis (15\%), and neurological conditions (14\%). Nearly half (45\%) witnessed a MOID 1-2 times per month and MOIDs associated with patient harm were noted by $79 \%$ at a frequency of $1-2$ times per year. $23 \%$ of respondents reported an absence of a systemic process of identifying MOIDs. Nearly half (45\%) reported fear of open discussion of MOIDs. More than half (55\%) felt that patients received unnecessary diagnostic tests in the pediatric ED and 42\% felt that patients suffer more harm from over-testing than from delayed/ missed diagnosis. Many respondents (44\%) suggested they rather over-test than accept diagnostic uncertainty at time of the patient encounter. Less than a quarter reported obtaining clinical reasoning training after completion of residency/fellowship training, with majority (80\%) suggesting that ongoing training in clinical reasoning should be an important part of their continuing education.

Conclusion: A global sample of pediatric ED physicians reported to experience harmful diagnostic errors relatively frequently, but many were uncomfortable discussing them. Despite recognizing the use of unnecessary diagnostic tests, several preferred over-testing than diagnostic uncertainty. Advancement of clinical reasoning skills should also target the balance between diagnostic uncertainty and over-testing.

\section{Communicating Diagnostic Uncertainty. Results from Observation of Master Clinicians and Patient Focus Groups}

\author{
M. Mirica ${ }^{1}$, A. Groisser, H. Haskell, P. Zuckerman, G. Schiff ${ }^{1}$
}

${ }^{1}$ Brigham and Women's Hospital, Boston, MA

Background: Uncertainty is a key concept in medicine. Culture of medicine resists acknowledging uncertainty. Few physicians and patients are taught about how to communicate with each other about diagnostic uncertainty, and there is a paucity of evidence/information about optimal ways do so.

Methods: We developed and tested a patient leaflet and accompanying physician guide to help guide communication around diagnostic uncertainty. The leaflet was developed based on literature reviews and interviews with master clinicians and further tested with 3 patient focus groups with. 9 master clinicians were presented with 4 clinical scenarios of uncertain diagnosis related to headache, cold symptoms, back pain, and enlarged lymph node, and were asked to think aloud through what their conversation with a patient in each scenario would sound like. 3 focus groups with 20 patients solicited feedback about the patient leaflet. Collected feedback was used to re-frame the tool. The new iteration of the tool will be tested in several primary care clinics. 
Results: The master clinicians' responses to the 4 clinical scenarios contained common themes: it was important for physicians to engage patients in the diagnostic process, communicate in a clear and transparent way, provide supportive, validating explanations and reassures, explain differential diagnosis and dynamic nature of disease, and operationalize the test of time. Patient focus groups were asked to provide feedback on the patient leaflet. While the patients believed that the bulk of the conversation about diagnostic uncertainty needed to happen verbally between the physician and the patient, most participants believed the leaflet was valuable as a supplemental educational tool. Based on their feedback, we reduced the length of the leaflet and restructured it to primarily focus on concrete actionable steps patients can take towards a more definitive diagnosis. An innovative one-page model was designed in coordination with two focus group patient advocates. The remainder of the tool contains brief educational information about diagnostic uncertainty and reiterate partnership between the doctor and the patient.

Conclusions: It is important for patients and their doctors to discuss diagnostic uncertainty, yet currently little is known about how to approach these difficult conversations. Both physicians and patients can benefit from a structured tool designed to assist in these discussions. Additional evidence is needed to understand how to incorporate such tools and conversations into a clinician's workflow best ways to engage both sides in the conversation and tailor patients differing health literacy levels and diagnostic challenges.

\title{
Improving Communication and Diagnosis in Cases of Medically Unexplained Symptoms
}

\section{O'Leary}

Rotman Institute of Philosophy, Western University, London, ON, Canada

Statement of the problem: Patients with medically unexplained symptoms (MUS) are central to everyday practice, and they are the group best known for patient-provider conflict. Research on MUS in medicine and psychiatry has failed to resolve these conflicts. In fact, among the general public, concern about MUS management is becoming more common, more organized and more intense. For the benefit of individual patients and providers, and for the benefit of the public's relationship with the medical profession, it's important to develop a new approach.

Findings to date: As an ethicist who works in this area, I advise patient advocacy groups concerned about management of MUS. In that capacity, I've come to understand patients' primary reason for initiating conflict: they are concerned that psychosomatic diagnosis for unexplained symptoms is often unsupported. Resolving this concern requires an honest evaluation of support for the cognitive leap from lack of medical evidence to psychological causes.

An examination of this kind reveals, first, that textbooks and practice guidelines encourage providers to conflate diagnostic uncertainty with psychosomatic diagnosis. In fact, because 'MUS' is ambiguous, providers lack terminology to draw a careful distinction between the two (O'Leary 2018). Second, we do not have criteria for psychosomatic diagnosis that have been shown to safely exclude unrecognized medical conditions (O'Leary 2019). Third, there is evidence that medical conditions are commonly mistaken for psychosomatic conditions in women (Dusenbery 2017), those with autoimmune disease (AARDA 2015) and those with rare disorders (Eurodis 2012). For these reasons, it is rational for patients to resist current management of MUS.

Description of the intervention or program: To improve communication and reduce diagnostic error, providers should: (1) recognize that diagnostic uncertainty is an acceptable and very common outcome of diagnostic inquiry; (2) recognize that it's wise for patients, and physicians, to resist conflation of diagnostic uncertainty and psychosomatic diagnosis; (3) develop strategies for alleviating personal discomfort with uncertainty, so that it's possible to discuss uncertainty candidly with patients; (4) develop a template for presenting patients with the range of diagnostic possibilities, and the risks involved in pursuing them, so that patient and provider can reach collaborative decisions about the best course of action.

Lessons learned: Patients' concerns about MUS management are well founded and should be heeded in practice. Doing so will reduce diagnostic error, improve patient-provider communication, and help bridge the growing divide between the public and the medical profession.

\section{Improving Practice with EMR Integrated Pathways: Standardizing Evidence Based Diagnosis and Practice}

\author{
S. Peterson, M. Saheed, S. Figueroa, C. Harbertson \\ Johns Hopkins University School of Medicine, Department of Emergency Medicine, Baltimore, MD
}

Introduction: Standardization has been shown to improve efficiency and safety of care that is provided to patients. This work integrates evidence based pathways into the EMR in order to both improve diagnosis and treatment supported by evidence in the literature with integrated tools for early and accurate identification of certain conditions.

Description: The AgileMD program was used integrating appropriate labs, imaging, medications and consultations directly into flow diagrams to maximize ease of use for each pathway which was subsequently directly integrated into the EMR with direct links to the supporting evidence. For certain pathways, such as hyponatremia or DKA, an algorithm was run in the background that would flag the provider with a Best Practice Advisory (BPA) recommending the use of a pathway when certain lab conditions were met (ex. low sodium, hyperglycemia with associated anion gap). 
A clinical operations based committee consisting of four clinicians, a nurse, a pharmacist, and informatics specialist met weekly to develop and review pathways based on the up to date accepted literature for a given indication. A feedback system was available for providers to raise questions which were reviewed at weekly meetings, and actions taken for resolution were sent.

Results: Since the initiation of the AgileMD program, 70 pathways were developed and are active in the system. Recent analysis demonstrates increased use of the pathways for hyponatremia and DKA pathways with the introduction of BPA's (See Figure 1) Additionally, low risk patients with DVT/PE were less likely to get admitted to the hospital following implementation of the DVT/PE pathway (18\% pathway used vs. 47\% admitted pathway not used) and were more likely to be appropriately prescribed DOAC medications- avoiding the need for continuous outpatient INR monitoring ( $76 \%$ vs. $27 \%)$.

Conclusions: Implementation of the AGILE program has been useful as an education tool in real time in addition to leading to measurable improvement in value based metrics for many of the pathways implemented as well as early diagnosis and treatment of critical conditions.

\title{
Use of Electronic Health Record to Identify Diagnostic Errors in Hospitalized Patients Requiring Rapid Response Team Activation
}

\author{
Y. Pinevich ${ }^{1}$, J. Soleimani ${ }^{1}$, A. Barwise ${ }^{1}$, Y. Dong ${ }^{1}$, V. Herasevich ${ }^{1}$, O. Gajic ${ }^{2}$, B. Pickering ${ }^{1}$ \\ ${ }^{1}$ Department of Anesthesiology and Perioperative Medicine, Mayo Clinic, Rochester, MN \\ ${ }^{2}$ Division of Pulmonary and Critical Care Medicine, Mayo Clinic, Rochester, MN
}

Background: Although the National Academy of Medicine has identified diagnostic error and delay (DEAD) as a leading cause of preventable harm, we lack the necessary measurement tools to recognize DEAD in real-time. The objective of this study is to determine the feasibility of identifying DEAD in hospitalized patients using data retrievable from the electronic medical record (EMR).

Methods: This prospective observational study includes all patients, aged 18 years and older with research authorization experiencing their first RRT activation at Mayo Clinic, Rochester from May 1, 2019 to present. Exclusion criteria include readmission within 30 days, and recurrent RRTs. A standard operating procedure (SOP) was developed to review the EMR in order to identify patients with DEAD, defined as the failure to identify or communicate an explanation for the current health problem within 6 hours of the patient's presentation (see below). This threshold was arbitrarily chosen as a reasonable amount of time in which patients, presenting to a tertiary care academic center, should have an accurate differential diagnosis communicated in the EMR. All charts were reviewed independently and in duplicate by two investigators. Clinical features, including vital signs, labs, images, orders, nursing flowsheets, and clinical documentation were assessed at different timepoints, including at admission, RRT event, 24 and 72 hours after RRT activation. We identified the reason for the RRT call and searched for evidence of a documented diagnostic explanation for the presentation. In the event that no diagnostic explanation was recorded pre-RRT, we searched for evidence that the RRT was due to a new clinical problem present $<6$ hours prior to RRT activation. We followed patients for up to 72 hours post-RRT looking for evidence of any documented new diagnosis. Any disagreements or uncertainty about whether DEAD occurred were resolved by a senior critical care clinician.

Results: A total of 389 patients had a RRT activation, of which 168 patients (male - 53.6\%; age $64.6 \pm 15.8$ years) were eligible for inclusion. Applying our SOP, DEAD was positively identified in $10.1 \%$ of eligible cases. Common diagnoses associated with DEAD included sepsis (47.1\%), shock (35.3\%), arrhythmias (11.8\%), and acute respiratory failure (5.8\%).

Conclusion: In this study we developed a SOP to identify DEAD in patients requiring RRT activation. To further validate our strategy, we are conducting surveys of clinicians involved in the care of the patient before, during and after the RRT assessing whether they believed DEAD contributed to the RRT.

\section{Ending Diagnostic Delays in Cancer}

\author{
A. Ramirez, M. Holt
}

Tanana Chiefs Conference, Fairbanks, AK

Statement of problem: Tanana Chiefs Conference is a consortium of 42 tribes and organizations responsible for the delivery of health care to over 18,000 American Indians and Alaska Natives in the Interior of Alaska, a region almost the size of the state of Texas. Comprehensive, coordinated primary, specialty and emergency care is delivered in Fairbanks, the regional hub, and in over 25 remote village settings, most of which are not on the road system. In 2015, tribal members and tribal leadership identified the need to address perceived delays in the diagnosis of cancer.

Description of the intervention or program: A multidisciplinary team of providers, a pharmacist, and case managers reviewed new diagnoses of cancer monthly to determine if a delay had occurred and identify opportunities for improvement. Data collected included the date of first presentation, date of diagnosis, stage, type of cancer, and whether the cancer was screening amenable. For each case that took longer than 30 days from presentation to diagnosis the team determined the primary reason for the prolonged time. Reasons were grouped into the following categories: Diagnostic referral, Diagnostic procedure, Abnormal finding follow up, Disease presentation, and Patient factors. 
Based on the reviews "close the loop" processes were implemented for all referrals for specialty consultation and diagnostic procedures. A periodic "Lessons Learned" session was created for the Medical Staff regarding learnings critical to medical practice. Case management was implemented for patients with a known diagnosis of cancer to ensure appropriate long term surveillance. A suspicious imaging registry was developed to ensure follow up of abnormal imaging lesions. Tribal leaders were engaged in intensified efforts to increase cancer screenings. Findings to date: Almost 200 cases have been reviewed to date.

The number of median days to diagnosis of cancer decreased by 60\% between 2016 and 2018. At the 2019 TCC Convention, annual meeting of TCC full board with attendance by over 300 tribal members, tribal members used the opportunity to express gratitude for the work done to improve cancer care.

Lessons learned: Though the review process intensely focused on cancer diagnoses the processes implemented improved the diagnostic process for all medical care.

Continued commitment to the cancer review process including routine sharing of the data has supported development of trust between tribal leaders and the medical care teams.

\section{Developing a Tool for Evaluation of Diagnostic Decision Making in Simulation}

$\underline{\text { I. Rasooly }}{ }^{1}$, E. Orenstein ${ }^{2}$, R. Grundmeier ${ }^{3}$, N. Muthu ${ }^{3}$

${ }^{1}$ The Department of General Pediatrics and The Department of Biomedical and Health Informatics, Children's Hospital of Philadelphia, Philadelphia, PA

${ }^{2}$ Children's Healthcare of Atlanta, Atlanta, GA

${ }^{3}$ The Department of General Pediatrics and The Department of Biomedical and Health Informatics, Children's Hospital of Philadelphia, Philadelphia, PA

Purpose: Electronic health record (EHR) based simulation, in which participants retrieve information from a simulated EHR, provides a training environment with fidelity to the clinical decision-making process. While simulation is an established educational strategy for the development of knowledge and skills, we lack tools to evaluate diagnostic decision making in simulation. We aim to develop a measure of diagnostic performance in electronic health record (EHR) based simulation.

Description of program and assessment: Since 2015, we have conducted hour-long, monthly sessions using an EHR simulation environment nearly identical in design and functionality to the one used in clinical practice. The simulation presents an infant with hyperbilirubinemia, frequently a physiologic condition with straightforward management but occasionally a harbinger of more ominous conditions. Participants often misdiagnose the simulated patient as having physiologic hyperbilirubinemia, missing vital signs and other indications of sepsis. During post-simulation discussion we introduce data visualization and data retrieval tools that might aid in diagnostic decision making.

A more precise understanding of deficits in the decision-making process that result in diagnostic error would allow facilitators to introduce targeted information processing tools or strategies. We used the Situation Awareness Global Assessment Technique (SAGAT), a human factors approach based on structured expert interviews, to develop an instrument which evaluates components of decision making: perception of relevant information, comprehension of what the information means, and projection of potential outcomes. We will use this framework to understand the basis of misdiagnosis and to introduce tools to promote accurate diagnosis.

Outcomes: We are currently piloting the evaluation tool in simulation sessions at two tertiary care children's hospitals and developing postsimulation teaching to address deficits in perception, comprehension, or projection that lead to misdiagnosis.

During the pilot, we will collect evidence of instrument validity by examining whether it can differentiate between novice and experienced clinicians. We will also survey session facilitators about tool acceptability. Ultimately, we will assess utility based on improved decision making in in subsequent simulation sessions.

Discussion: We previously demonstrated that EHR tools introduced in simulation have broad, sustained adoption. We are now testing the feasibility of using an instrument derived from human factors approaches in EHR simulations to assess diagnostic performance.

Significance: In an era of complex EHR based information, clinicians require information processing strategies and tools to formulate accurate diagnosis. We propose a strategy for using simulation to evaluate and address deficits in diagnostic decision making. 
Sample Instrument

\begin{tabular}{lll}
\hline Facilitator Questions & $\begin{array}{l}\text { Participant answers that demonstrate } \\
\text { diagnostic accuracy }\end{array}$ & $\begin{array}{c}\text { Underlying Element of } \\
\text { Decision Making }\end{array}$ \\
\hline What is the patient's last blood pressure? & - Not recorded & Perception \\
What is the patient's last heart rate? & $->200$ & Perception \\
What is the heart rate trajectory? & - Increasing & Comprehension \\
& - Worsening, despite fluids & Comprehension \\
What is the overall ED assessment? & - Now rehydrated 4-day old baby with likely & Comprehension +/- \\
& physiologic jaundice & Projection \\
Is there further evaluation or intervention & - Yes; based on discussion & \\
the ED should consider? & - Yes; full sepsis evaluation including blood & cultures and starting antibiotics + fluids \\
& - Yes; sepsis workup & Projection \\
Do the data match the ED assessment? & - No & - Potentially; after further discussion with the ED \\
Is the patient ready for admission? &
\end{tabular}

\section{Frequency of Diagnostic Errors in the Neonatal Intensive Care Unit: A Retrospective Cohort Study Using the Safer Dx-NICU Instrument}

\section{G. Shafer ${ }^{1}$, H. Singh ${ }^{2}$, G. Suresh ${ }^{1}$}

${ }^{1}$ Department of Pediatrics, Baylor College of Medicine/Texas Children's Hospital, Houston, TX

${ }^{2}$ Center for Innovations in Quality, Effectiveness and Safety, Michael E. DeBakey Veterans Affairs Medical Center, Baylor College of Medicine, Houston, TX

Background: Diagnostic errors remain understudied in the Neonatal Intensive Care Unit (NICU). The few available studies are autopsy-based, which thus excludes diagnostic errors not resulting in death. We determined the frequency of diagnostic errors for inborn (i.e. born at our institution) NICU patients using medical record reviews.

Methods: We conducted a retrospective medical record review of 600 consecutive inborn admissions to the NICU (average daily census of 145) at a quaternary care hospital from January 1, 2017 - July 20, 2017. We used a data collection tool - an adapted version of the Safer Dx Instrument - with questions to evaluate diagnostic processes and identify the presence/absence of diagnostic errors. A trained NICU physician used the instrument to review the electronic medical record for the first 7 days of admission, and categorized cases as "yes," "unclear," or "no" for diagnostic error. After all charts underwent primary review, we used a two-step process to facilitate adjudication: (1) A blinded secondary review of all charts in the "yes" category plus a random sample of charts in the other two categories, conducted by one of 4 trained NICU physician leaders; and (2) Non-blinded group review by all four secondary reviewers of charts where there was discordance between primary and secondary reviews.

Results: Primary review thus far has identified 50 patients with a diagnostic error (8.3\% of patients reviewed, with 1.1 errors per 100 patient days). Additionally, the primary review categorized 14 patients as "unclear," and 536 as not having any diagnostic error. Secondary reviews are ongoing. Common types of process breakdowns involved history and exam (Table 1). There were 11 different types of diagnoses involved in the errors. Infectious conditions were most common (36\%) followed by renal conditions (14\%). In 4 patients, diagnostic errors likely led to death. Conclusions: Use of a standardized data collection instrument has identified a high frequency of NICU diagnostic errors of various types and origins. We are now completing secondary reviews and further results are forthcoming.

Table 1: Frequency of Diagnostic Errors Grouped by Main Etiology $(n=50)$

\begin{tabular}{lr}
\hline Etiology of Diagnostic Error & $\boldsymbol{n}$ \\
\hline Misinterpreted or Missed Maternal History Findings & 27 \\
Incomplete or Incorrect Physical Exam & 11 \\
Misinterpreted or Missed Laboratory Study Results & 7 \\
Misinterpreted or Missed Radiologic Study Results & 4 \\
Missed Equipment Malfunction & 1 \\
\hline
\end{tabular}




\title{
The Clinical Problem Solvers - A Diagnostic Reasoning Podcast
}

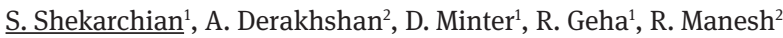 \\ ${ }^{1}$ University of San Francisco School of Medicine, San Francisco, CA \\ ${ }^{2}$ Johns Hopkins University School of Medicine, Baltimore, MD
}

Purpose: The rise of social media has transformed the way we communicate. A systematic review (PMID 23619071) concluded that interventions using social media tools were associated with improved knowledge, attitudes, and skills. These platforms, particularly Twitter and podcasts, have become increasingly popular in the medical community.

Clinical reasoning is fundamental to the practice of medicine, but this complex process is rarely modeled as teachable habits. Rather, it is often developed through trial and error. The landmark Improving Diagnosis in Health Care report identified the lack of focus on developing clinical reasoning as a "major gap in education within all health care professions" which then contributes to diagnostic error.

The gap identified in clinical reasoning education and the far-reaching power of social media inspired us to create The Clinical Problem Solvers (CPSolvers) - an internal medicine podcast dedicated to diagnostic reasoning through a case-based, iterative process.

Description: The CPSolvers is a free weekly podcast, featuring two types of episodes:

1. Schema based: focus on teaching a systematic approach to commonly encountered problems through patient cases (e.g., abdominal pain) and

2. unknown case: a blinded discussant is presented a clinical case and shares her clinical reasoning extemporaneously.

Our website (clinicalproblemsolving.com) has downloadable versions of discussed schemas and videos which walk the viewer through the majority of our schemas.

Outcomes: Since the launch of CPSolvers in December 2018, we have produced 11 schema based and 27 unknown episodes. Eleven of these unknown episodes were done in collaboration with the Human Diagnosis Project. CPSolvers attracts 5,500 listeners per episode in 137 countries. Our website has over 100,000 visits to date and our products have been used as curriculum at multiple institutions worldwide.

Discussion/Significance of findings: We highlight the worldwide reachability of a podcast dedicated to diagnostic reasoning.

\section{Addressing Cardiovascular Disease Risk: Integrating Clinical Decision Support and Patient Education into Physician Workflow through an Autocalculating Atherosclerotic Cardiovascular Disease Risk Tool}

\author{
$\underline{\text { L. Stein }}^{1}$, L. Schubel ${ }^{1}$, R. Romero Barrientos ${ }^{1}$, Z. Hettinger ${ }^{1}$, M. Townsend ${ }^{2}$, P. Basch ${ }^{1}$, W. Weintraub ${ }^{1}$, K. Chaney ${ }^{3}$, K. Miller ${ }^{1}$ \\ ${ }^{1}$ MedStar Health, Washington, D.C. \\ ${ }^{2}$ Centra Health, Lynchburg, VA \\ ${ }^{3}$ Office of the National Coordinator for Health IT (ONC), Washington, D.C.
}

Statement of problem: Physicians utilize validated risk calculators such as the American College of Cardiology's atherosclerotic cardiovascular risk (ASCVD) calculator to aid in clinical decision making and to engage patients. However, current workflow requires physicians to take valuable time to google it, and then to manually enter patient information for the calculation. Consequently, this useful tool is underutilized due to its lack of integration into physician workflow. By simultaneously engaging physicians and developers, our researchers were able to develop and an interoperable ASCVD risk calculator that functions within the electronic health record (EHR).

Description of the intervention or program: The purpose of our research is to pilot a tool that provides a clear and comprehensive method of communication for cardiovascular risk in a user-friendly, efficient platform that supports all stakeholders including cardiologists, primary care physicians, and patients. Our prototype was developed through user centered and iterative design to incorporate known limitations inherent to human cognition while preserving strengths of experienced clinicians.

Findings to Date: The Mobilizing Million Hearts tool includes two separate interfaces: the static ASCVD Risk Estimator and the dynamic ASCVD Risk Educator. The Risk Estimator will present to the physician immediately upon opening a patient chart. This is intended as a quick reference to convey a patient's 10-year ASCVD risk. The Risk Educator is designed as a visual aid to augment risk education provided to patients. The physician can manipulate values to illustrate how 10-year ASCVD risk may change with certain medical or lifestyle interventions. It can also serve as a decision-making aid for clinicians (e.g. decision to order a statin). A key element of both is that they pull patient information directly from the EHR, eliminating the need for physicians to interrupt their workflow to search for, and manually enter, patient information in order to receive a calculation. Another key element is interoperability: the tool is designed to use Substitutable Medical Applications, Reusable Technologies (SMART) on Fast Healthcare Interoperability Resources (FHIR) technology so that it is replicable across multiple EHR platforms. 
Lessons Learned: It is vital to engage both end-user and application developer stakeholders in the design of interoperable clinical decision support tools. Input from clinicians as well as developers is key to ensuring mutual understanding throughout the design process. Next steps include formative usability testing to optimize the tool interface to support clinical intervention and ensure usability, sophistication, and actionable clinical decision support.

\title{
Implementation of an Institutional Review Process to Identify Targets for Improving Diagnostic Safety from Risk Management Data
}

\author{
$\underline{\text { D. Upadhyay }}^{1}$, J. Hunt ${ }^{1}$, S. Korukonda ${ }^{1}$, D. Torretti² ${ }^{2}$ H. Singh ${ }^{3}$ \\ ${ }^{1}$ Safer Dx Learning Lab, Quality and Safety, Geisinger, Danville, PA \\ ${ }^{2}$ Geisinger Medical Center, Geisinger, Danville, PA \\ ${ }^{3}$ Center for Innovations in Quality, Effectiveness and Safety, Michael E. DeBakey Veterans Affairs Medical Center and Baylor College of \\ Medicine, Houston, TX
}

Statement of problem: Most health care organizations do not have comprehensive mechanisms to identify and learn about diagnostic safety from risk management data.

Description of the intervention: We created an institutional program for data collection and analysis that included a diagnostic safety champion, an institutional 'Committee to Improve Diagnosis' and a team of 3 individuals (two MDs and one RN) who analyzed available institutional safety data. Safety data included cases reported by risk management and physicians piloting our EHR-based staff messaging system. The team was trained by diagnostic safety researchers in series of meetings that helped pilot tools to determine diagnostic errors and their contributory factors. This consensus-based review approach was used to study 91 diagnostic errors out of 120 reported cases from 2017 and led to a shared mental model on understanding diagnostic error. For all subsequently reported cases, each reviewer performed individual independent review and analysis using the Revised Safer Dx instrument and the Safer Dx Process Breakdown Supplement.

Findings to date: From June 2018 till date, 147 cases with potential missed opportunities in diagnosis (MODs) were reported, of which 131 cases have been analyzed thus far (16 cases still under review). MODs were found in 61 cases which were analyzed using the lens of the Safer Dx Framework process dimensions. Most cases ( $\mathrm{n}=46 ; 35 \%)$ involved breakdown in the patient-provider interaction (history/physical exam/ assessment); others involved diagnostic test interpretation $(n=22 ; 15 \%)$ and follow-up and tracking dimensions ( $n=22 ; 15 \%)$. Among the 61 MODs, top five conditions were related to cancers (17 cases), sepsis (5), fractures (5), bowel perforation (3) and strokes (3). Shortness of breath, abdominal pain and headache were the most common symptoms associated with MODs. Most MODs occurred in the ED (25 cases), followed by outpatient setting (14), inpatient (8), radiology (7) and pathology (4).

Lessons Learned: We developed a review process to analyze institutional risk-management data for missed opportunities in diagnosis, previously applied only in research settings. As we transitioned to a continuous practice improvement model, reporting of cases from risk management increased, as did cases without MODs. Initial insights reveal several opportunities for improving diagnosis, specifically related to breakdowns in data gathering/interpretation within the patient-provider encounter. Other health care systems could similarly create institutional review and analysis programs for assessment of risk management data and identify contributory factors to generate learning for improvement.

\section{Quantifying the Medical Student Learning Curve for ECG Rhythm Strip Interpretation using Deliberate Practice}

L. Waechter ${ }^{1}$, D. Reading 2 , M. Walker ${ }^{3}$, C. Hee Lee ${ }^{1}$

${ }^{1}$ University of Calgary, Calgary, AB, Canada

${ }^{2}$ University of British Columbia, Vancouver, BC, Canada

${ }^{3}$ University of McGill, Montreal, QC, Canada

Objectives: Obtaining competency in medical skills such as interpretation of electrocardiograms (ECGs) requires repeated practice and feedback. Structured repeated practice and feedback for ECGs is likely not provided to most medical students, so skill development is dependent on opportunistic training during clinical rotations. Our aim was to describe: 1) the amount of deliberate practice completed for learning ECG rhythm strip diagnoses in first year medical students, 2) the learning curve for rhythm strip diagnosis, and 3) student experiences with deliberate practice.

Methods: First year medical students from two medical schools were provided with online rhythm strip practice cases. Diagnostic accuracy was measured throughout practice, and students were provided feedback for every case they completed. Total cases practiced and time spent practicing were correlated with their performance during practice and on an exam. 
Results: 314 of 384 (82\%) students consented. The mean number of ECGs each student practiced was 59 (range 0-280), representing 18,466 total instances of deliberate practice. We generated mathematical models that accurately correlated both the number of cases practiced and time spent practicing, with diagnostic accuracy on an exam $(p<0.001)$. For example, students would need to spend on average of 112 minutes and complete 34 practice cases to obtain $75 \%$ on an ECG rhythm strip exam. Student satisfaction was high using the online cases.

Conclusions: We succeeded in delivering deliberate practice for ECG rhythm strip interpretation to a large cohort of students at 2 medical schools. We quantified a learning curve that estimates the number of cases and practice time required to achieve pre-determined levels of diagnostic accuracy. Comparing our data with previously collected data from the Clerkship Directors of Internal Medicine (CDIM) survey in 2005 suggests that little formalized structured ECG practice occurs; it is possible that practice-feedback opportunities are far below the needs of the medical students at many medical schools in North America. Our data can help inform a competency-based approach to curriculum development.

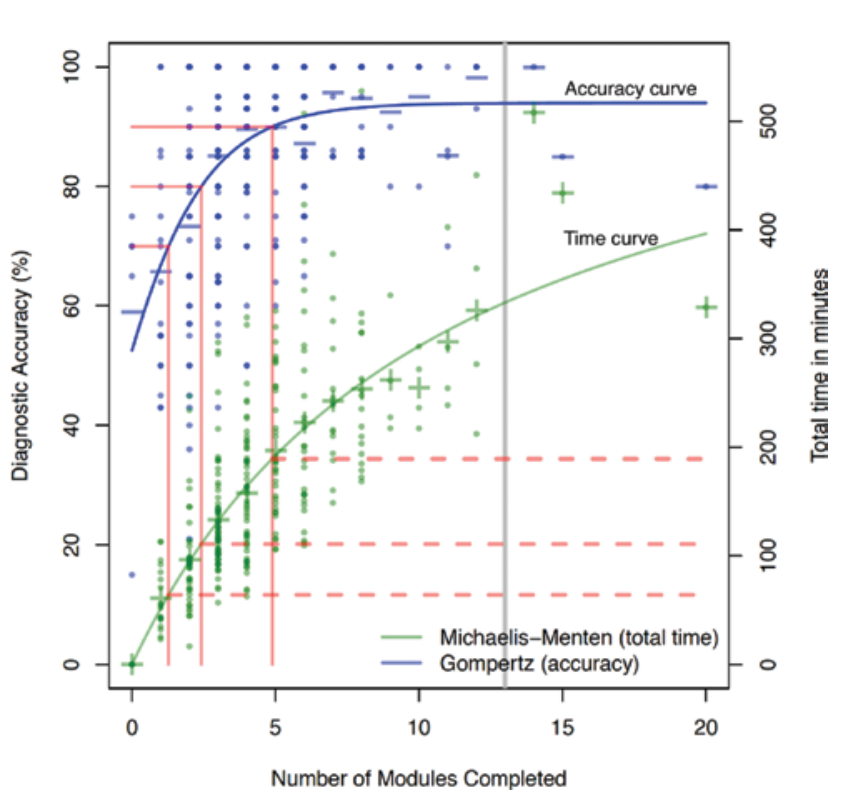

Development and Validation of an Oncology-Specific Trigger Tool

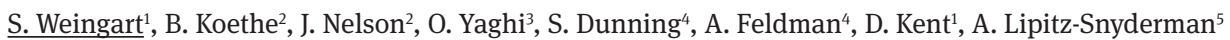

${ }^{1}$ Tufts Medical Center and Tufts University School of Medicine, Boston, MA

${ }^{2}$ Tufts University School of Medicine, Boston, MA

${ }^{3}$ Tufts Medical Center, Boston, MA

${ }^{4}$ OptumLabs, Cambridge, MA

${ }^{5}$ Memorial Sloan Kettering, New York, NY

Background: "Trigger tools" specify clinical markers in the medical record that suggest the possibility of treatment complications or diagnostic delays. Though used widely in health care, the development of oncology-specific triggers has been elusive due to patients' high symptom burden. To address this challenge, the authors developed and tested a set of oncology-specific triggers using diagnosis and treatment codes in a large commercial claims database. Methods: We selected subjects with a new diagnosis of breast, colorectal, lung, or prostate cancer treated with an initial course of cancer-directed therapy from 20008-14. Data were abstracted from the OptumLabs data warehouse. Using diagnosis and procedure codes, we defined 15 oncology-specific triggers drawn from a pilot study, all with PPVs $>50 \%$. Triggers included events such as neutropenic fever, use of contact precautions, return to the operating room, or initiation of therapeutic anticoagulation. To distinguish carerelated complications from comorbidities, we required a causal and temporal relationship between treatment and trigger. We tabulated the prevalence of triggers by cancer type and metastatic status during a one-year follow up period across all sites of care and created multivariate logistic regression models to examine the association between cancer triggers and mortality. 
Results: The cohort of 322,887 unique patients included 27\% with metastatic disease. Triggers were prevalent in the cohort, affecting as many as $40.4 \%$ of patients with metastatic colorectal cancer and $50.2 \%$ of patients with metastatic lung cancer. The most common triggers included blood transfusions, hypoxemia, contact precautions, and chest imaging following radiation therapy. The mortality rate was substantially higher among patients with at least one trigger compared to patients with none. Experiencing at least one cancer-specific trigger increased the one-year odds of death for all cancer types, and by as much as 2.28 (95\% CI 1.73-3.00) for non-metastatic prostate cancer.

Conclusions: Oncology-specific triggers provide researchers a promising method for identifying errors and adverse events in cancer care.

Trigger prevalence within 1 year, by cancer type and metastatic status

\begin{tabular}{|c|c|c|c|c|c|c|c|c|}
\hline \multirow[t]{2}{*}{ Trigger } & \multicolumn{4}{|c|}{$\begin{array}{l}\text { Non-metastatic } \\
n=236,456\end{array}$} & \multicolumn{4}{|l|}{$\begin{array}{l}\text { Metastatic } \\
\mathrm{n}=86,431\end{array}$} \\
\hline & $\begin{array}{l}\text { Breast } \\
n=97462\end{array}$ & $\begin{array}{l}\text { Colorectal } \\
n=33712\end{array}$ & $\begin{array}{l}\text { Lung } \\
n=21142\end{array}$ & $\begin{array}{l}\text { Prostate } \\
n=84140\end{array}$ & $\begin{array}{l}\text { Breast } \\
n=26791\end{array}$ & $\begin{array}{l}\text { Colorectal } \\
n=18671\end{array}$ & $\begin{array}{l}\text { Lung } \\
n=30169\end{array}$ & $\begin{array}{l}\text { Prostate } \\
n=10800\end{array}$ \\
\hline Any trigger & $10.2 \%$ & $19.2 \%$ & $32.8 \%$ & $5.9 \%$ & $30.9 \%$ & $41.4 \%$ & $50.2 \%$ & $24.6 \%$ \\
\hline Anticoagulation & $0.1 \%$ & $0.2 \%$ & $0.3 \%$ & $0.1 \%$ & $0.2 \%$ & $0.5 \%$ & $0.4 \%$ & $0.2 \%$ \\
\hline Bacteremia/positive blood culture & $0.3 \%$ & $1.1 \%$ & $1.6 \%$ & $0.3 \%$ & $1.4 \%$ & $3.1 \%$ & $2.4 \%$ & $1.6 \%$ \\
\hline Abnormal serum bicarbonate & $1.8 \%$ & $3.9 \%$ & $4.7 \%$ & $1.9 \%$ & $5 \%$ & $9.3 \%$ & $7.3 \%$ & $6.3 \%$ \\
\hline Blood transfusion & $1.9 \%$ & $2.6 \%$ & $12.2 \%$ & $0.6 \%$ & $8.8 \%$ & $9.6 \%$ & $23.2 \%$ & $7.6 \%$ \\
\hline C. difficile positive & $0.3 \%$ & $1.1 \%$ & $1.3 \%$ & $0.2 \%$ & $1.1 \%$ & $2.5 \%$ & $1.8 \%$ & $0.7 \%$ \\
\hline Non-contrast chest CT following XRT & $1.6 \%$ & $2.1 \%$ & $10.3 \%$ & $1.1 \%$ & $3 \%$ & $4.1 \%$ & $11.3 \%$ & $4.9 \%$ \\
\hline Elevated creatinine & $0.1 \%$ & $0.3 \%$ & $0.6 \%$ & $0.1 \%$ & $0.5 \%$ & $0.8 \%$ & $1 \%$ & $0.4 \%$ \\
\hline Hypoxemia/ low oximetry & $1 \%$ & $2.8 \%$ & $12.2 \%$ & $1.2 \%$ & $4 \%$ & $5.9 \%$ & $16.3 \%$ & $4.6 \%$ \\
\hline Contact precautions/isolation & $6 \%$ & $4.1 \%$ & $6.2 \%$ & $1.2 \%$ & $9.5 \%$ & $6 \%$ & $8 \%$ & $3.6 \%$ \\
\hline Nasogastric tube & $<11$ events & $0.5 \%$ & $<11$ events & $0.1 \%$ & $<11$ events & $0.6 \%$ & $<11$ events & $<11$ events \\
\hline Nephrology consult & $0.3 \%$ & $1.4 \%$ & $1.3 \%$ & $0.6 \%$ & $1.3 \%$ & $3.1 \%$ & $2.1 \%$ & $2.6 \%$ \\
\hline Neutropenic fever & $4 \%$ & $4.5 \%$ & $6.7 \%$ & $0.6 \%$ & $6.8 \%$ & $5.7 \%$ & $7.4 \%$ & $2.6 \%$ \\
\hline Percutaneous drain & $0 \%$ & $0.8 \%$ & $0.6 \%$ & $0.3 \%$ & $0.1 \%$ & $2.4 \%$ & $0.5 \%$ & $0.6 \%$ \\
\hline Abnormal serum potassium & $2.5 \%$ & $7.3 \%$ & $8.1 \%$ & $1.8 \%$ & $8.7 \%$ & $17.7 \%$ & $13.9 \%$ & $6.9 \%$ \\
\hline Press ulcer & $0.3 \%$ & $1.2 \%$ & $1.0 \%$ & $0.2 \%$ & $0.6 \%$ & $1.5 \%$ & $0.9 \%$ & $0.8 \%$ \\
\hline Reoperation/ return to operating room & $0.1 \%$ & $2.4 \%$ & $0.4 \%$ & $0.6 \%$ & $0.2 \%$ & $3.7 \%$ & $0.4 \%$ & $0.7 \%$ \\
\hline
\end{tabular}

XRT, radiation therapy. Values shown in the table are prevalence rates of patients with a trigger within an exposure window among those with the relevant treatment exposure. 
Explaining the Diagnosis: A Tool for Patients and Doctors.

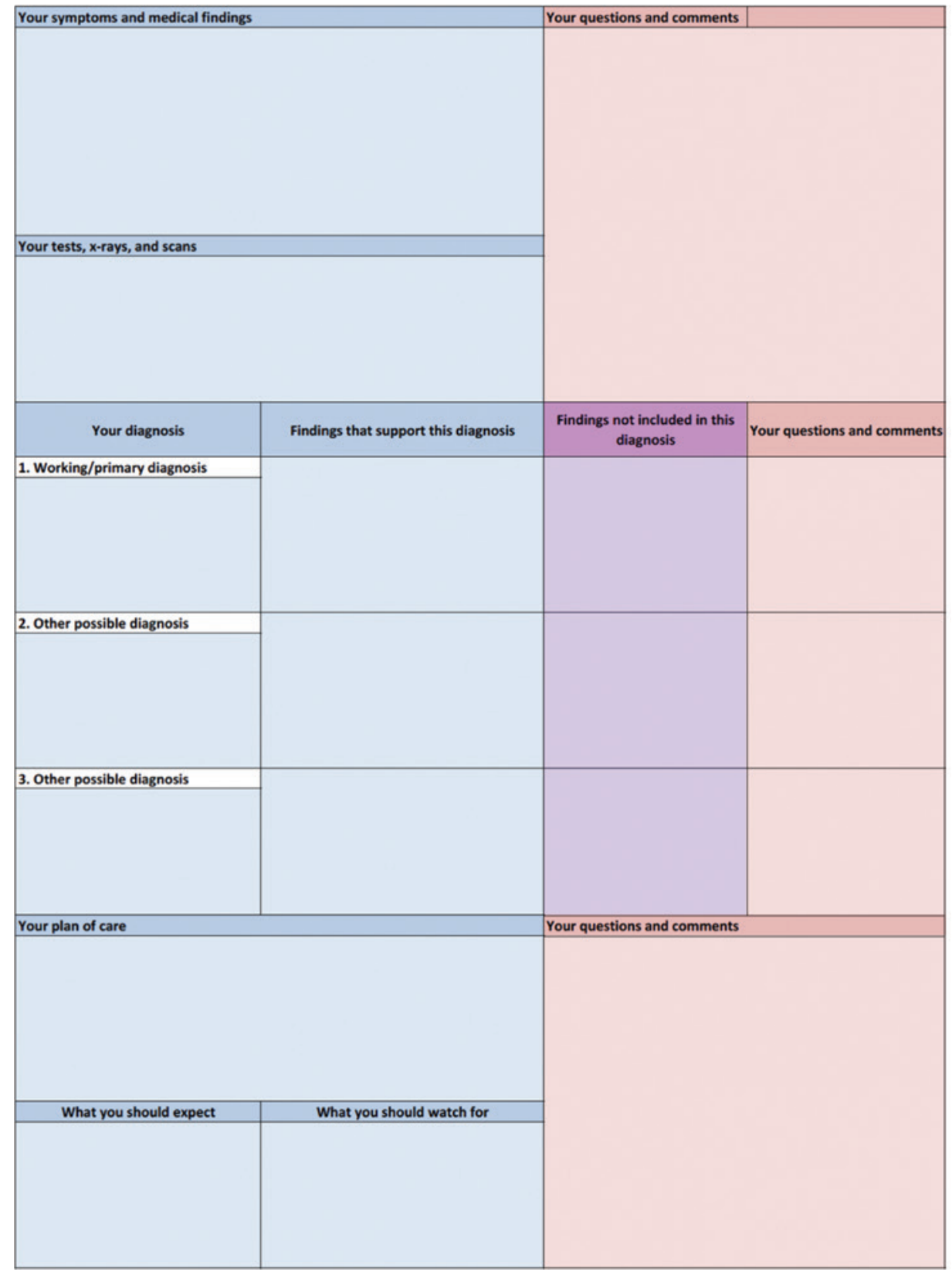




\section{Poster Session 1 \\ Monday, November 11 \\ 3:15 PM - 4:15 PM}

\section{Scientific}

\section{Does Communication Matter? Invalidation of Patient Symptoms During the Diagnostic Process and its Effects on Self- Esteem and Depression}

\section{A. C. Bontempo}

Rutgers University

Background: Qualitative research has investigated the diagnostic experience of women with endometriosis. Women frequently report the difficulty they experienced getting healthcare providers to acknowledge a physical problem was responsible for their somatic complaints (hereafter referred to as symptom invalidation [SI]). Some of such research has also reported that SI by physicians contributed to reductions in patient self-esteem, particularly prior to their eventual endometriosis diagnosis. The aim of this research was to empirically validate the association between SI and self-esteem observed by extant qualitative research as well as to 1) examine the influence of patients' message internalization in this relationship and 2) examine these variables' influence on depression as an outcome.

Methods: Participants were 563 patients with self-reported endometriosis who participated in a larger online research study. SI and internalized SI were measured with scales developed for this research. Self-esteem was assessed using the Rosenberg Self-Esteem Scale and depression was assessed using the Center for Epidemiologic Studies-Depression scale. Multiple hierarchical multiple regressions were performed, and path analysis was performed to test for mediation. Demographics and pain served as covariates in all models.

Results: SI was only slightly associated with self-esteem $(\beta=-.09, \mathrm{p}<.05)$ and not with depression $(\beta=.06, \mathrm{p}=.18)$. However, internalized SI was associated with both self-esteem $(\beta=-.12, p<.01)$ and depression $(\beta=.09, p<.05)$. The path analysis model testing the effect of internalized SI on depression through self-esteem was significant, demonstrating full mediation.

Conclusion: These results confirm qualitative research and provide the first quantitative evidence that SI, particularly when internalized, is associated with reduced self-esteem and greater depression. Despite limitations, these results highlight the importance of internalization in SI and the potential of targeting internalization as a psychotherapeutic target. Furthermore, these results demonstrate that SI is not benign at the psychological level in this sample.

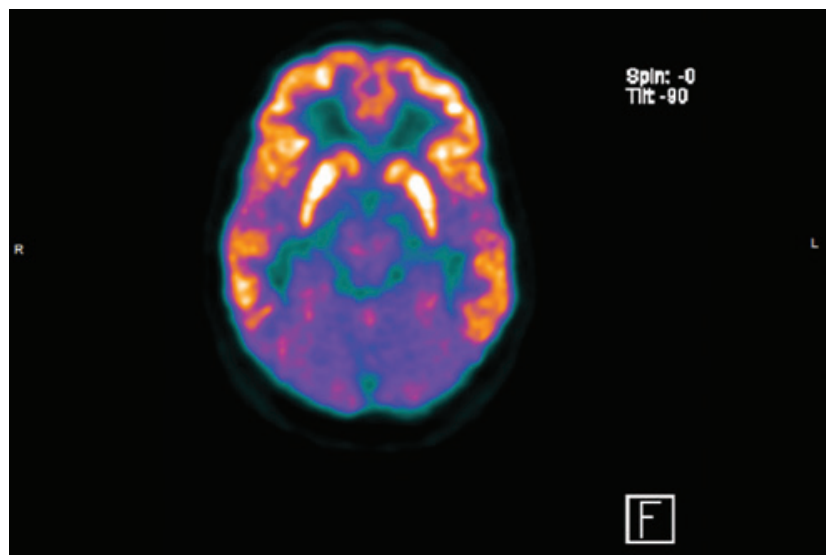




\title{
Absence of Evidence is not Evidence of Absence: Symptom Misattributions Stemming from Null Nondiscriminatory Diagnostic Testing for Endometriosis
}

\author{
A. C. Bontempo
}

Rutgers University

Background: HCPs, when they communicate to endometriosis patients their lack of an endometriosis diagnosis, they often make certain (incorrect) attributions about the origin of their somatic complaints, ones that patients consistently report are invalidating. This research thus sought to identify these attributions.

Methods: Using an anonymous online survey for which patients were recruited via social media sites of endometriosis organizations, participants $(\mathrm{N}=822)$ from the US and abroad were asked to describe a specific interaction with a HCP that occurred prior to diagnosis during which they felt they or their symptoms had been dismissed.

Results: The first and predominant type of attribution is that patients' somatic experiences are part of normal physiology, most commonly menstruation, but also pre-menstrual syndrome, ovulation, perimenopause or menopause, and pregnancy, and that these patients are either outliers or have a low tolerance to pain. A second type is that patients' somatic experiences are psychological in one of two ways. First, their experiences are believed as real but are attributed to physical manifestations of psychological pain. Second, their experiences are not believed as real and are attributed to other psychological factors such as anxiety/depression leading to misinterpretation of their symptoms, as being "all in the head" or imaginary, and as attention-seeking. A third type is those of other physical health diagnoses, most commonly STIs/STDs/PID, among others. In many cases, attributions were made following null test results of nondiscriminatory diagnostic testing, such as blood tests, ultrasound, CT, and MRI. This is important for two reasons. First, the only way to definitively diagnose endometriosis is through laparoscopy with biopsy. Although generalists may be unaware of these diagnostic guidelines, OB/GYNs, as specialists, should-but often are not-aware. Therefore, HCPs appear overhasty in attributing null test results to normal physiology or to psychological factors. Second, as per the APA's DSM-5, a diagnosis of a mental health disorder is not valid if it is based solely on the absence of physical evidence for somatic complaints.

Discussion: It is crucial for HCPs-especially OB/GYNs-to be trained in the proper diagnosis of endometriosis, especially due to its common $(10 \%)$ prevalence rate and $50 \%$ comorbidity rate with infertility. It is also imperative that 1$)$ HCPs become appropriately trained in mental health if they indeed make so many (mis)diagnoses of these conditions, and/or that 2) they refer such patients to trained mental health specialists to rule out psychopathology as the origin of symptoms.

\section{Learning by Example or Learning by Concept: An Experimental Study of Bayesian Diagnosis}

\author{
J. E. Brush, M. Lee, J. Sherbino, J. C. Taylor-Fishwick and G. R. Norman \\ Virginia Medical School and Sentara Healthcare and McMaster University
}

Background: The psychology and medical literature suggests that when people (and clinicians) are provided with new information and asked to revise their judgments, they are either conservative - not changing the probability sufficiently compared to Bayes' theorem - or wildly error-prone. These findings appear in the literature under multiple labels such as base rate neglect, representativeness, and confirmation bias. Optimizing Bayesian reasoning is a goal of medical education, but specific teaching methods have not been tested.

Methods: In this study, we examined learners' (61 third and fourth-year medical students) ability to correctly revise disease probability on receipt of test results. During the learning phase, students were randomized to 1 of 3 conditions: 1. Examples, where students worked through 9 cases involving each of 3 diagnostic categories - pulmonary embolus, congestive heart failure and acute coronary syndrome - and were given feedback on the final probability, 2. Video, where students reviewed a video discussing concepts of base-rates, sensitivity, specificity, and likelihood ratios then were given 1 worked example per diagnostic category, and 3. Control, where students read about the 3 diagnostic categories. At test, students viewed written cases containing clinical findings and were asked to provide a pretest probability of diagnosis. Then they received the test result (positive or negative) and were asked to provide a revised post-test probability. All students were tested on 20 cases - 4 new cases per diagnostic category, 4 cases of a new diagnostic category and 4 fillers. The outcome was a computed difference score based on comparison between their revised post-test probability estimate and a Bayesian calculation of post-test probability using their pretest probability.

Results: The Video condition showed a significant advantage: Using an Effect Size measure (where a large value indicates greater error), means for each condition were: Video $=.336, \mathrm{SE}=.034$, Examples $=.446, \mathrm{SE}=.035$, Control $=.484, \mathrm{SE}=.036,(\mathrm{~F}=4.94, \mathrm{p}<.01)$. The advantage of Video also held for the new cases although it did not achieve significance. Interestingly, the discrepancies between subjective estimates and Bayesian calculations were small in all groups - an average of 1.5\% for Video, 3\% for Examples and 4\% for Control.

Conclusion: The results show that teaching students a conceptual framework for Bayesian updating significantly improves the accuracy of post-test probability estimates. In addition, the results showed that participants in all groups were surprisingly better at Bayesian updating than suggested by prior studies in the psychology and medical literature. 


\title{
Unplanned Emergency Department Re-attendances - An Evaluation of Emergency Care
}

\author{
L. Q. Chiu, I. B Silvestre, D. L. Elumba, J. H. Cheng Thay, V. Wing Yin Siu and H. Ang
}

Background: Unplanned Emergency Department (ED) re-attendances may suggest potential shortcomings in care. This study aims to describe the incidence, reasons and outcomes for these unscheduled visits.

Methods: We conducted a retrospective study of ED patients who presented between 1 April 2018 and 31 March 2019 and who re-attended within $72 \mathrm{~h}$. Patient demographics, ED diagnosis, re-attendance reason, final disposition, and length of stay were recorded.

Results: The re-attendance rate was 3.9\%. The median age was 51 and 63.6\% were males. Top reasons for re-attendance were disease progression (55.9\%), presentation with an unrelated condition (15.6\%), and psychiatric issues (9.8\%). Only 1.8\% of patients returned due to missed diagnoses. $28.3 \%$ of re-attendances were admitted and had a median length of stay of 3 days. Most frequent reasons for admission were disease progression (85.4\%), presentation with an unrelated condition (4.1\%), and non-compliance with medical therapy (3.3\%). There were 5 mortalities. Disease progression resulted in 2,277 re-attendances, with musculoskeletal complaints (26.6\%), infections (19.1\%) and abdominal complaints $(17.3 \%)$ contributing to the majority. Complaints relating to the spine and limbs contributed to $81.6 \%$ of musculoskeletal complaints and upper respiratory tract complaints made up almost half of infections leading to re-attendance.

Conclusion: The re-attendance rate is comparable to local and regional data but higher than international rates. Adverse outcomes are few. This data serves as a pilot study to focus future ED efforts at managing re-attendances. Further qualitative studies should be done to evaluate the most frequent reasons of re-attendances as described above.

Footnote: Reasons for re-attendance were classified as follows: social fragility, psychiatric issues, missed clinical condition, iatrogenic causes, disease progression, return after discharge by inpatient teams, non-compliance with medical therapy, alcohol-related ED visits, presentation with an unrelated condition, and administrative reasons.

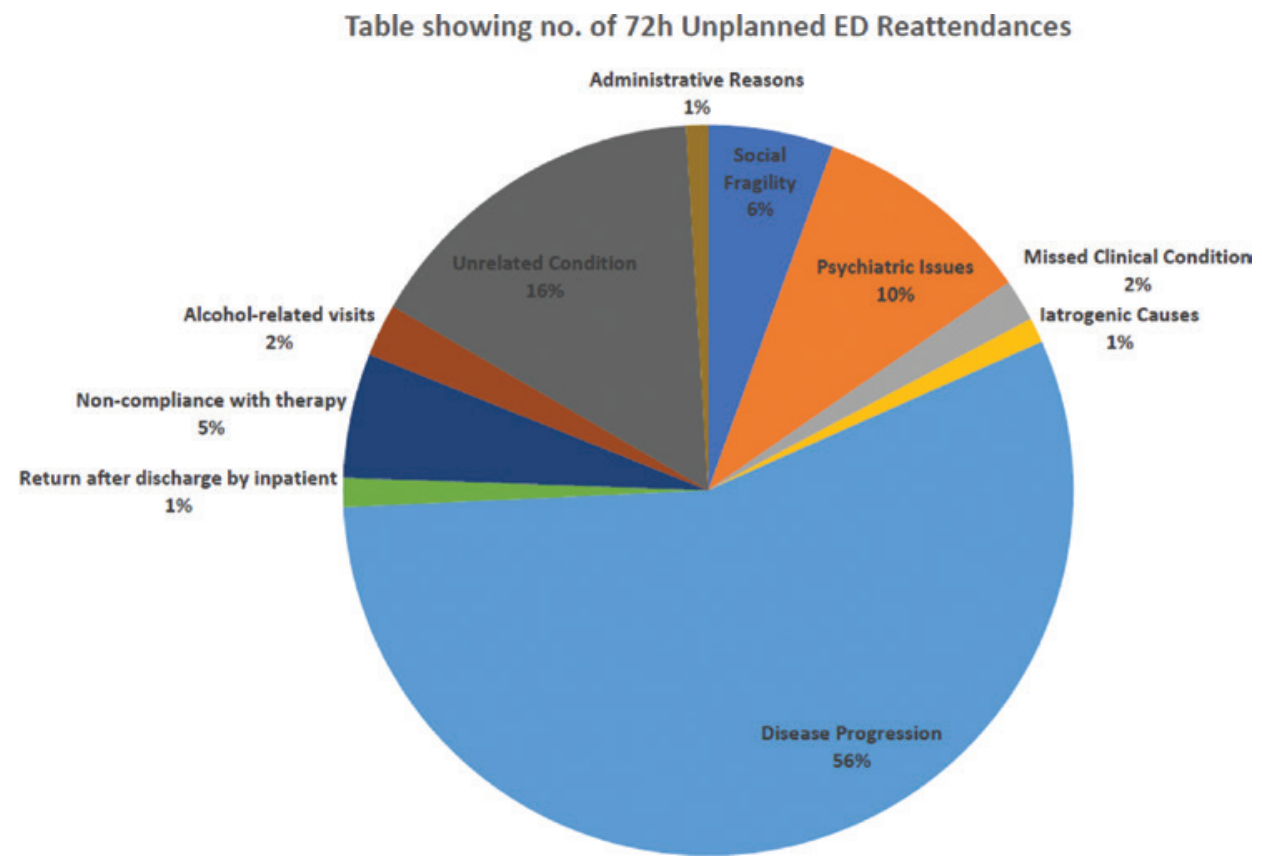

\section{Correlations Between Inpatient Physician Admitting Shift Busyness, Diagnostic Confidence, and Physician Cognitive State}

$\underline{\text { A. Gupta }^{1,2}}$, M. Rogers' ${ }^{2}$ S. Winter ${ }^{2}$, K. E. Fowler ${ }^{1}$, T. McCarty ${ }^{1}$, S. Saint ${ }^{2}$ and V. Chopra ${ }^{1,2}$

${ }^{1}$ VA Ann Arbor Healthcare System

${ }^{2}$ Division of Hospital Medicine, Department of Medicine, University of Michigan Medical School

Background: Little is known about the interplay between shift busyness, diagnostic confidence, and cognitive state of physicians admitting patients to the hospital.

Methods: On weekdays between September and December 2018, inpatient physicians at a tertiary-care academic medical center were assessed during their 8-hour afternoon admitting shift (swing shift). Physicians were equipped with a Spire StoneTM - a novel biometric device that monitors time spent tense, calm, and focused based on the subject's breathing pattern. A survey was administered to physicians 
at the end of the shifts to characterize confidence level in each admitted patient's diagnosis ( 1 = not at all confident; $10=$ most confident). Patient medical records were reviewed and data on lab test and imaging study ordering were recorded. Records on patterns of pages received by physicians were obtained. For analyses, random-effects linear regression models were used for panel data, accounting for multiple swing shifts for each physician. Alpha was set at 0.05, 2-tailed. Analyses were conducted in Stata/MP 15.1 .

Results: A total of 25 inpatient physicians admitted 74 patients over 41 swing shifts. Reported mean diagnostic confidence was 7.7 (SD 1.9). On average, participants spent 55 minutes in a calm state (range: 4 to 164) and 40 minutes in a tense state (range: 2 to 186) per shift. Physicians spent more time in a tense state at the end of the week compared to the beginning of the week $(p=0.009)$. As the number of imaging tests ordered increased, the number of calm minutes increased $(p=0.002)$ and the number of tense minutes decreased $(p=0.009)$. The mean number of pages per shift for physicians who saw one patient was 22.8 (SD 8.1), increasing to 30.8 (SD 11.9) pages per shift for physicians seeing three patients $(\mathrm{p}=0.036)$. There was no significant association between the number of minutes spent in the tense state and number of pages on the shift, number of patient admissions, diagnostic confidence level, or total number of labs ordered. There were more tense minutes for physicians during the first and fifth hours of the shift (Figure).

Conclusion: Inpatient physicians spent more time in the tense state at the beginning and end of the shift. Obtaining imaging studies appears to reduce the amount of time spent in the tense state but effect on diagnostic accuracy and patient outcomes is unclear. Future research to evaluate the true impact of physician cognitive state and busyness on diagnostic outcomes appears necessary.

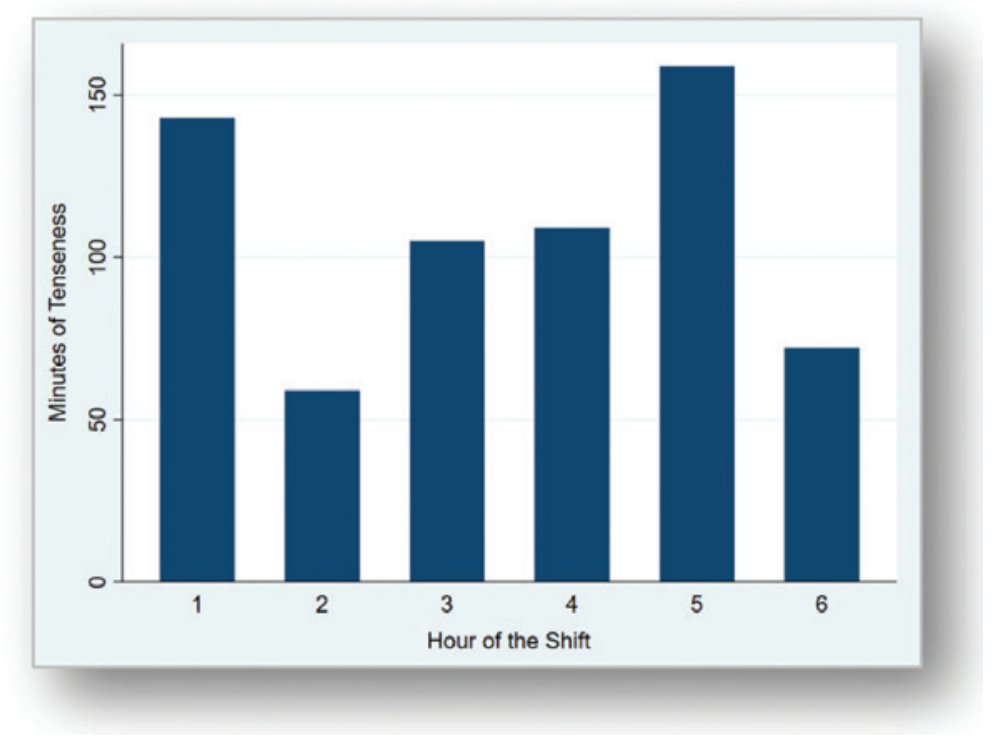

FIGURE. Number of Tense Minutes by the Hour of the Shift

\section{Diagnostic Confidence in the Inpatient Setting}

$\underline{\text { A. Gupta }}^{1,2}$, M. Rogers' ${ }^{2}$ S. Winter ${ }^{2}$, K. E. Fowler ${ }^{1}$, D. Glynn ${ }^{2}$, S. Saint ${ }^{1,2}$ and V. Chopra ${ }^{1,2}$

${ }^{1}$ VA Ann Arbor Healthcare System

${ }^{2}$ Division of Hospital Medicine, Department of Medicine, University of Michigan Medical School

Background: Little is known about behaviors associated with diagnostic confidence in the inpatient setting.

Methods: Inpatient physicians at a tertiary-care academic medical center were assessed during their 8-hour afternoon admitting shift occurring weekdays between September and December of 2018. A survey was administered to physicians at the end of their shifts asking, "How confident were you for the diagnoses of the patients you admitted today?” on a scale from 1 (not at all confident) to 10 (most confident) for each admission. Data including number of lab tests and imaging studies were collected via chart review. Records on volume and timing of pages received by physicians were obtained. Patients were excluded if they were admitted with a pre-specified diagnosis or were transferred from another unit. To account for the multiple swing shifts for each physician, random-effects linear regression for panel data was used to test for associations between diagnostic confidence (outcome) and study variables. Two-sided tests were used with $a=0.05$. Analyses were conducted in Stata/MP 15.1. 
Results: Over the course of 41 shifts, 25 inpatient physicians admitted 74 patients. Physicians saw an average of 2 patients (range 1-4, SD =0.9) per shift. The mean diagnostic confidence level was 7.7 (SD 1.9). Diagnostic confidence did not vary by gender ( $p=0.132$ ), or by "LACE” index $(\mathrm{p}=0.997)$ which incorporates length of stay, admission acuity, comorbidities and ED visits. Lower physician diagnostic confidence was associated with a greater number of lab tests $(\mathrm{p}=0.004)$ and imaging studies $(\mathrm{p}=0.024)$. For every 1 unit higher in diagnostic confidence, the mean number of lab tests decreased by 0.5. Diagnostic confidence increased throughout the day; the later in the day the patient was evaluated, the greater the reported diagnostic confidence of the physician $(\mathrm{p}=0.014)$. The data suggested a threshold effect when a physician saw at least 3 patients per shift; the diagnostic confidence declined $(p=0.007)$ when the number of pages increased with the third patient. For the third patient, the mean diagnostic confidence level decreased by 0.4 for each additional 5 pages. For the first two patients, this association was not evident. Conclusion: Decreased diagnostic confidence is associated with increased lab and imaging utilization, potentially reflecting physician uncertainty. Diagnostic confidence was correlated with timing of admissions, with higher confidence for later admitted patients. After the third admission, diagnostic confidence decreased, and paging volume increased. Limiting admissions to three patients or providing support for additional evaluations may help prevent diagnostic errors.

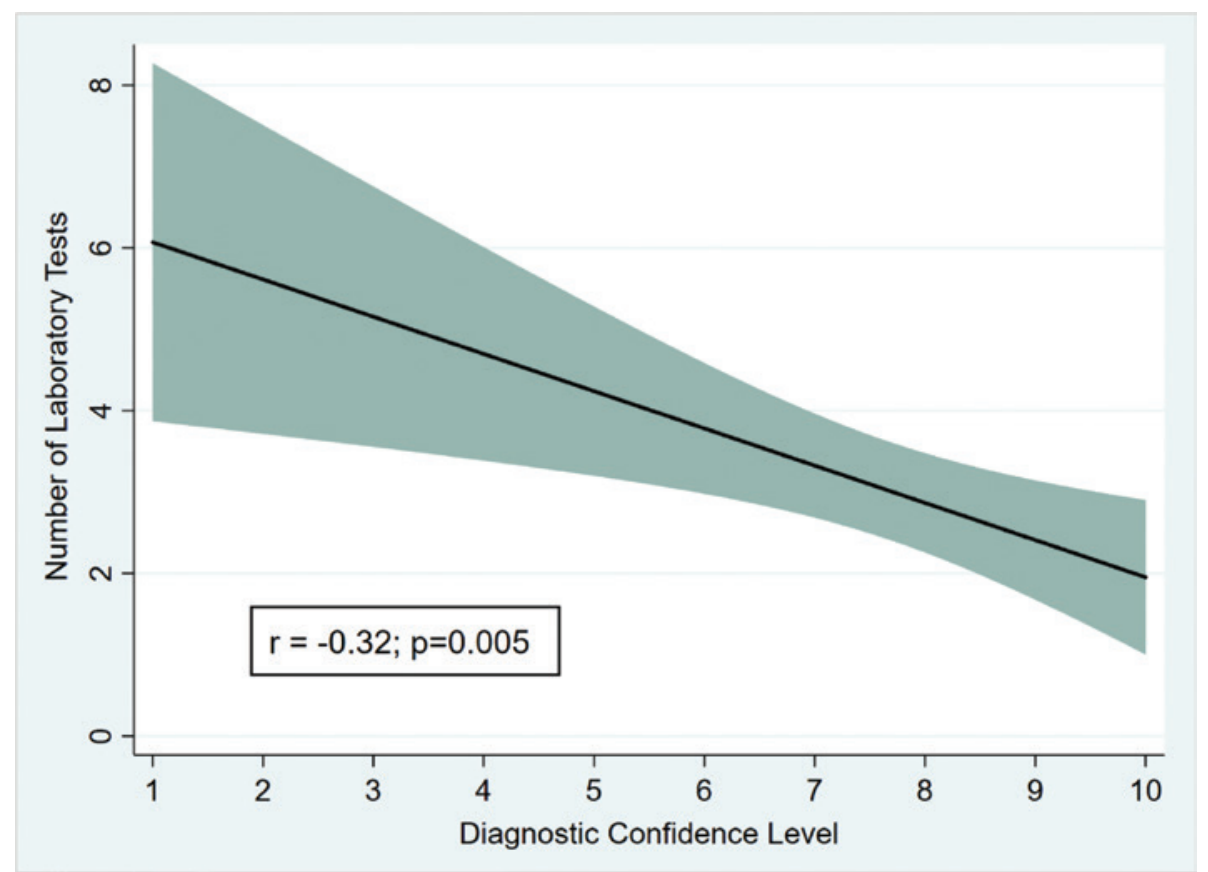

FIGURE: Mean number of laboratory tests ordered based on diagnostic confidence level

\title{
Migraine is a Great Risk Factor for Delay Diagnosis of Vertebral Artery Dissection: A Retrospective Study
}

\author{
T. Harada
}

Division of General Medicine, Showa University Koto Toyosu Hospital

Background: Vertebral artery dissection (VAD) is the most common single etiology for stroke in young adults, but its initial diagnosis is often difficult and easily misdiagnosed. The association between migraine and VAD may be at risk for diagnostic errors, but there are no clear data Method: Retrospectively analysis performed on 34 cases of vertebral artery dissection diagnosed from January 2014 to December 2018 at Showa University Koto Toyosu Hospital.

Result: Patient mead age is $54.5 \pm 13.1$, male was 50\%. Delay diagnosis occurred in 19 of 34 cases of VAD. The delay diagnosis was 2 cases within 1d, 2 cases within 1-3d, 7 cases within 4-7d, 8 cases after 1w. Most diagnostic delays were after 3 days. There were 5 cases with migraine headache, all cases had delayed diagnosis. Age, gender, diabetes, dyslipidemia, hypertension, smoking history, and absence of neurological symptoms were not associated with delayed diagnosis.

Conclusion: Migraine may be a great risk factor for delayed diagnosis of VAD. 


\title{
A Diagnostic Time-Out to Improve Differential Diagnosis in Pediatric Abdominal Pain
}

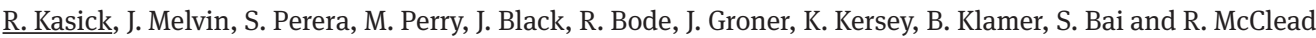 \\ Nationwide Children's Hospital and The Ohio State University, Columbus, $\mathrm{OH}$
}

Introduction: Pediatric abdominal pain is challenging to diagnose and often results in unscheduled return visits to the emergency department. External pressures and diagnostic momentum can impair physicians from thoughtful reflection on the differential diagnosis. We implemented a diagnostic time-out intervention and created a scoring tool to improve the quality and documentation rates of differential diagnosis.

Methods: Charts were aggregated using ICD-10 codes for abdominal pain. We reviewed a total of 165 encounters for patients admitted to the general pediatrics service at one institution. Sixty-four H\&Ps were reviewed during the baseline period, July-Dec 2017 ; 101 charts were reviewed post-intervention Jan-Jun 2018. Medical teams performed a diagnostic time-out intervention on all patients admitted to the general pediatrics service during the study period. Percent of initial H\&Ps, including 'complete' differential diagnosis (defined by inclusion of at least 2 working diagnoses and consideration of at least 1 life-threatening diagnosis) and quality scores using our Differential Diagnosis Scoring Rubric (Likert scale 0-5; a score of 5 representing an excellent and robust differential diagnosis) were tracked monthly.

Results: At baseline, 43 (67\%) resident notes, and 49 (77\%) attending notes documented a 'complete' differential diagnosis. Post-intervention, 59 ( $58 \%, p=0.26$ compared to baseline) resident notes and $69(68 \%, p=0.25)$ attending notes met this criteria. After the implementation of the diagnostic time-out, the mean number of diagnoses documented by resident physicians increased from $4.9 \pm 3.2$ to $5.1 \pm 3.4$ $(p=0.77)$. Similarly, the number of diagnoses documented by attending physicians increased from $6.2 \pm 3.5$ to $6.3 \pm 4.3$ ( $p=0.84$ ). There was no significant change in mean quality scores from pre- to post-intervention for resident-documented differential diagnoses (2.41 to 2.47 , $\mathrm{p}=0.73$ ) nor attending-documented differential diagnosis ( 2.85 to $2.82, \mathrm{p}=0.88$ ). Post-intervention, seven separate patients accounted for 13 unplanned 30-day readmissions for recurrent abdominal pain. Further analysis revealed more frequent documentation of 'complete' differential diagnosis on index admissions (residents 60.2\%; attendings 69.3\%) compared to subsequent readmissions within 30-days (residents $46.2 \%$; attendings $61.5 \%$ ). The average quality score was also higher for index admissions (residents 2.5; attending 2.9) compared to 30-day readmissions (residents 2.1; attendings 2.7).

Conclusions: We implemented a diagnostic time-out intervention and created a novel scoring rubric to assess the quality of differential diagnosis for pediatric abdominal pain. We demonstrated a marginal improvement in the quality of resident-documented differential diagnosis. Expansion of diagnoses considered within a differential diagnosis may contribute to higher diagnostic accuracy but may not reduce readmission rates.

\section{Update on Diagnostic Error Trends and Taxonomies Through the Lens of Malpractice Claims}

\author{
A. J. Lim ${ }^{1,2}$, D. E. Norwich ${ }^{1,2}$, S. M. Dulgarian 1 , S. Agarwal ${ }^{1}$, L. A. Volk ${ }^{1}$, C. Keohane ${ }^{4}$, D. Siegal ${ }^{4}$ and G. D. Schiff ${ }^{1,3}$ \\ ${ }^{1}$ Brigham and Women's Hospital, Boston, MA \\ ${ }^{2}$ Tufts Medical School, Medford, MA \\ ${ }^{3}$ Harvard Medical School, Cambridge, MA \\ ${ }^{4}$ CRICO Strategies, Boston, MA
}

Background: Medical malpractice records represent a rich source of diagnostic error cases and insights. Though not without limitations, they provide a concentrated view of patients who have experienced adverse outcomes related to diagnostic errors, and it has been reported that the leading cause of primary care malpractice cases are diagnosis-related1. Here we examine an aggregated database comprised of cases from multiple malpractice insurers. We sought to cull recent cases of diagnostic error claims, update earlier studies, identify new trends, and further understand the types and causes of errors occurring in primary care today by applying three novel taxonomies along with a previously widely-used and tested taxonomy that further illuminate types and patterns of errors.

Methods: Using CRICO's National Comparative Benchmarking System (CBS), a robust claims database that represents over 30 percent of medical malpractice cases from over 500 hospitals in the U.S., we compiled and categorized a sample of 312 diagnosis error-related claims closed between the years 2016 - 2018. We reviewed each case summary and classified each case using the DEER (Diagnosis Error Evaluation and Research), RDC (Reliable Diagnosis Challenges), Pitfalls, and Cognitive Errors taxonomies and used these data, in addition to CRICO's taxonomy, to conduct further analysis.

Results: Of the initial 312 cases collected, 53 were excluded due to either a lack of case narrative or for falling outside the scope of diagnostic error, leaving 259 cases for analysis and taxonomy classification. Analyses revealed most frequent diagnoses and taxonomy codes, which will be presented and discussed in greater detail (Table 1). Seven from among the analyzed cases have already been used in case discussions for the PRIDE Learning Network, which conducts modified M\&Ms with a group of cross-institutional experts of diverse backgrounds, with additional cases targeted for future discussions.

Conclusion: Medical malpractice claims serve as an extremely useful ongoing source of data and insights into the realities of medical error occurrences. Our analyses give clear indication of some of the most commonly-occurring errors, which are very similar to prior diagnosis and contributing factors, suggesting continuing efforts will need to be concentrated in these areas for further improvement and research. 
Table 1: Five most commonly occurring results in diagnostic and taxonomy coding categories

\begin{tabular}{|c|c|c|c|c|c|c|c|c|c|}
\hline \multirow[t]{2}{*}{ Top 5 Diagnoses } & \multicolumn{9}{|c|}{ n Top 5 Taxonomy Codes } \\
\hline & & DEER & n & RDC & $\mathbf{n}$ & Pitfalls & & $\begin{array}{l}\text { Cognitive } \\
\text { Error }\end{array}$ & \\
\hline $\begin{array}{l}\text { Malignant neoplasm } \\
\text { of breast }\end{array}$ & 16 & $\begin{array}{l}\text { Failure/delay in } \\
\text { ordering needed test }\end{array}$ & 143 & $\begin{array}{l}\text { Recognition of } \\
\text { acuity/urgency/ } \\
\text { severity }\end{array}$ & 79 & $\begin{array}{l}\text { Inadequate follow-up } \\
\text { visits/referrals, } \\
\text { especially in the } \\
\text { presence of diagnostic } \\
\text { uncertainty }\end{array}$ & 84 & $\begin{array}{l}\text { Premature } \\
\text { closure }\end{array}$ & 67 \\
\hline $\begin{array}{l}\text { Malignant neoplasm of } \\
\text { bronchus/lung }\end{array}$ & 9 & $\begin{array}{l}\text { Failure/delay in } \\
\text { considering the } \\
\text { diagnosis }\end{array}$ & 102 & $\begin{array}{l}\text { Diagnosis of underlying } \\
\text { cause }\end{array}$ & 67 & $\begin{array}{l}\text { Failure in follow-up of } \\
\text { abnormal/critical result }\end{array}$ & 83 & Anchoring & 63 \\
\hline $\begin{array}{l}\text { Acute myocardial } \\
\text { infarction }\end{array}$ & 9 & $\begin{array}{l}\text { Failure in weighing } \\
\text { critical piece of } \\
\text { history data }\end{array}$ & 85 & Test follow-up issues & 64 & $\begin{array}{l}\text { Failure to monitor, note } \\
\text { or respond to evolving/ } \\
\text { continuing/ } \\
\text { persistent symptoms }\end{array}$ & 81 & $\begin{array}{l}\text { Base-rate } \\
\text { neglect }\end{array}$ & 55 \\
\hline Pneumonia & 9 & $\begin{array}{l}\text { Failed/delayed } \\
\text { follow-up of } \\
\text { (abnormal) test result }\end{array}$ & 72 & Patient failure to follow-up & 38 & $\begin{array}{l}\text { Urgency of the clinical } \\
\text { situation was not } \\
\text { appreciated }\end{array}$ & 76 & $\begin{array}{l}\text { Search } \\
\text { satisfying }\end{array}$ & 40 \\
\hline Pulmonary embolism & 8 & $\begin{array}{l}\text { Failure/delay to } \\
\text { recognize/weigh } \\
\text { urgency }\end{array}$ & 71 & $\begin{array}{l}\text { Performance/interpretation } \\
\text { challenges }\end{array}$ & 30 & $\begin{array}{l}\text { Failure to appreciate } \\
\text { risk factor (or those at } \\
\text { risk) for a given disease }\end{array}$ & 74 & $\begin{array}{l}\text { Availability } \\
\text { bias }\end{array}$ & 27 \\
\hline
\end{tabular}

Citations: CRICO. (2019). Retrieved from https://www.rmf.harvard.edu/Products-and-Services/CRICO-Strategies-Products-and-Services/CBS

\section{Self-Reported Learning (SRL), a Voluntary Incident Reporting System Experience within a Large Health Care Organization}

$\underline{\text { L. Lurvey }}^{1}$, M. Fassett ${ }^{1}$, M. Kanter ${ }^{2}$, M. Hugh ${ }^{1}$ and M. $\mathrm{Au}^{1}$

${ }^{1}$ Southern California Permanente Medical Group

${ }^{2}$ Kaiser Permanente School of Medicine

Background: Physicians remain largely unaware of their own error-causing potential and how their actions can prevent errors. Clinical peer review as a tool enhances accountability but does not encourage insight into the cause of errors or system thinking and physicians may feel the review is punitive. Using a model well established in aviation, we began a self-reported learning program in a large health care organization with the hypothesis that immunity from peer review would encourage self-reporting.

Methods: A system was set up to allow physicians to report their own errors. Physicians were surveyed after implementation. An analysis of all self-reported learning (SRL) submissions was conducted by physician quality leaders familiar with clinical peer review. They analyzed the submissions for evidence of secondary insight that might drive personal or system safety improvements to patient care.

Results: Findings in the survey showed that physicians overwhelmingly do not believe they make errors (70\%). Under SRL, the physicians showed some secondary insight as to how to avoid their errors in the future (40\%). Secondary insight regarding diagnostic errors included consulting with specialists to confirm a diagnosis, reviewing imaging studies and not just relying on the radiologist's report, assessing and considering the patient's past visit history, social situations and life stresses, and documenting differential diagnoses. Although total submissions were low, SRL also filled the emotional need to discuss errors (27\%) as much as avoiding peer review (27\%).

Conclusion: An SRL incident reporting system offers physicians a way to acknowledge errors, explore their contribution to bad outcomes, and avoid the embarrassment of having those errors brought to light. SRL alone doesn't raise reporting significantly but it does offer a useful and productive adjunct to clinical peer review for the prevention of errors and patient harm. 


\title{
Types of Diagnostic Errors Self-Reported by an International Cohort of Pediatric Emergency Physicians
}

\author{
$\underline{\text { P. Mahajan }}^{1}$, J. A Grubenhoff ${ }^{1}$, J. A. Cranford ${ }^{1}$, K. N. Shaw ${ }^{3}$, J. M. Chamberlain ${ }^{4}$, R. M. Ruddy ${ }^{5}$, A. Belle ${ }^{1}$, N. Kuppermann ${ }^{6}$ and H. Singh $^{7}$ \\ ${ }^{1}$ Department of Emergency Medicine, University of Michigan \\ ${ }^{2}$ University of Colorado School of Medicine, Department of Pediatrics, Section of Emergency Medicine \\ ${ }^{3}$ Division of Pediatric Emergency Medicine, Department of Pediatrics, University of Pennsylvania, Children's Hospital of Philadelphia \\ ${ }^{4}$ Division of Pediatric Emergency Medicine, Department of Pediatrics, Children’s National Medical Center \\ ${ }^{5}$ Department of Pediatrics, University of Cincinnati College of Medicine, Cincinnati Children's Hospital Medical Center \\ ${ }^{6}$ Departments of Emergency Medicine and Pediatrics, University of California-Davis School of Medicine \\ ${ }^{7}$ Center for Innovations in Quality, Effectiveness and Safety, Michael E. DeBakey Veterans Affairs Medical Center, Baylor College of Medicine
}

Background: Diagnostic errors, reframed as missed opportunities for improving diagnosis (MOIDs), in pediatric emergency departments (EDs) are poorly understood. Practicing physicians could provide unique insights. To study the epidemiology, harm, and contributing factors related to MOIDs self-reported by ED physicians participating in a consortium of global pediatric ED research networks called the Pediatric Emergency Research Networks (PERN).

Methods: We developed a two-part, web-based survey and pilot tested it before administering it within PERN. In Part 1 (presented here) physicians described specific examples of MOIDs they experienced involving their own patient or a patient of their colleague. Respondents provided a brief case summary and answered questions regarding resulting harm and factors contributing to the event as mapped to the process dimensions of the Safer Dx framework.

Results: Of 1208 physicians, $267(22.1 \%)$ responded ( $\mathrm{M}$ age $=48$ years [SD =9.9], 56\% female, mean years in practice $=13$ [SD =9.7]). Many reported patient presentations involving MOIDs had common undifferentiated symptoms, including abdominal pain, headache, and fever. Often, the affected patients were discharged from the ED with commonly occurring pediatric diagnoses (e.g. constipation, bronchiolitis, asthma, viral infections). Most reported MOIDs (65\%) were detected on ED return visits (50\% within 24 hours and $89 \%$ within 72 hours). The final correct diagnoses sometimes included life-threatening conditions such as myocarditis, cancer, physical abuse, and meningitis. The responding provider was solely involved in 25\% and involved with other ED team members in 38\% of MOIDs. Most respondents (92\%) reported that there was a clear opportunity to make the right diagnosis at the initial index ED encounter. Important contributing factors to MOIDs reported related to: the patient-provider encounter (61\%), diagnostic tests (40\%), patient-related factors (28\%), follow-up and tracking (14\%) and consultations (10\%). More than half (54\%) of the reported MOIDs involved the patient-provider encounter, with misinterpreted/ignored history or an incomplete/inadequate physical examination. More than half of involved patients had moderate (48\%) or major (10\%) harm. Conclusions: Approximately one in five pediatric ED physicians from a global cohort responded with rich details regarding a patient who experienced a diagnostic error. Our study suggests that these errors occur in children who present with common childhood complaints and many of these errors share common contributory factors across practice settings and countries. Physicians' personal experiences offer an under-explored source for investigating and mitigating diagnostic error.

\section{The Frequency of Abnormal Blood Test Results Associated with Increased Cancer Risk and Their Follow-up in Australian General Practice}

\section{P. Nguyen, J. McIntosh and J. Emery}

University of Melbourne

Background: Diagnostic errors are relatively common in primary care and can arise from missed or delayed diagnoses such as delays in the follow-up of abnormal test results that suggest potential cancer risk. This can be concerning as missed cancer is one of the leading categories of harmful diagnostic errors in primary care. However, potential and early signs of cancers can first present in primary care with common, non-specific symptoms or a combination of symptoms. Cancer is also relatively uncommon in primary care, despite being a frequently identified condition for diagnostic errors. As general practitioners (GPs) have a significant role as gatekeepers to specialist care in Australia, this presents challenges for GPs to make appropriate and timely actions and potential diagnoses for cancer. The aim of this study is to investigate and quantify the frequency of abnormal pathology test results that are associated with increased cancer risk and the time for general practitioners to follow up these results in Australia.

Methods: This retrospective study will involve secondary analysis of the Australian NPS MedicineInsight dataset, a longitudinal, de-identified primary care dataset. We will be examining all available data from 2007 to 2017 and includes over 60 million individual pathology test results. The prevalence of abnormal test results will be measured to determine how common the abnormalities are.

We will also include the analysis of linked state hospital datasets, the Victorian Emergency Minimum Dataset (VEMD) and the Victorian Admitted Episodes Dataset (VAED). We will investigate high-level interactions between primary care and hospital data to identify patients presenting to hospital care (i.e. Emergency Department). This will allow us to capture follow-up and referrals from GPs in the hospital outpatient dataset that are not well captured in the NPS MedicineInsight primary care dataset alone.

Results: This study makes use of the Australian NPS MedicineInsight dataset and the advancement of large-scale, data linkage between primary care data, hospital data and cancer registry data through the Victorian Comprehensive Cancer Centre Health Data Platform in 
Australia. Analysis is expected to commence in August 2019 with final results expected by November 2019. Results will be presented at the DEM2019 conference.

Conclusion: This will be the first study to quantify the prevalence of abnormal test results and their follow-up in Australia. Findings from this study will also inform and contribute to subsequent research which aims to improve the management of abnormal test results and early cancer diagnosis in primary care.

\title{
System Engineering Framework and Simulation for Diagnostic Error in a Hospital Setting
}

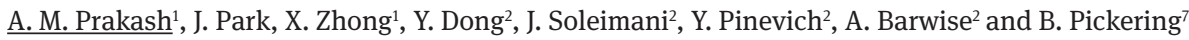 \\ ${ }^{1}$ University of Florida \\ ${ }^{2}$ Mayo Clinic Rochester
}

Background: Diagnostic error (DE) is an emergent property of a complex healthcare system with contributions from various stakeholders, tools, processes, and their interactions, necessitating a system based investigation. The objective of this study is to a) develop a systems framework to identify factors and bottlenecks causing DE and b) develop mixed method models to design and evaluate system based interventions.

Methods: A Systems Engineering Initiative for Mitigating Diagnosis Error (SEIMDE) framework is adapted from the Systems Engineering Initiative for Patient Safety (SEIPS) 2.0 framework based on the operations at Mayo Clinic, Rochester. Literature review, process observations, interviews, stakeholder focus groups were conducted to develop the framework. Based on the critical components identified from the framework, a multi-method simulation model is then developed to simulate hospital operations which are composed of various agents and processes. We propose a hierarchical modeling approach that allows us to understand the interactions between agents, including patients, providers, artifacts (such as information) and processes, and their overall impact on the system. We start with building unit level models to fully capture the interactions between patients, information flow, and providers. Then, several unit level subsystems are assembled to create the system level model. The model accommodates patient heterogeneity and health transitions, care team functions such as intervention administration, communication, their interaction with data tools and their internal cognitive models, and interruptions that arise in the system at significant levels of detail. Optimization and machine learning algorithms will be used to identify system based interventions.

Results: The SEIMDE framework provides a tool to systematically identify, analyze and rate factors related to patient, provider, tasks, technology, external and internal environment, and their interactions that contribute to DE. The simulation model captures various clinical processes, patient health transitions, and physician's decision making. It offers a platform to perform experiments to identify bottlenecks within the system and to test the impact of process interventions on patient outcomes. The model design will be subject to scrutiny by physicians and validated through reconciliation with electronic data.

Conclusion: Descriptive and prescriptive systems analysis of DE will be aided qualitatively by the SEIMDE framework and quantitatively by the simulation model built upon the SEIMDE framework in a closed loop approach. The approach provides a foundation on which process improvement interventions can be tested and where future machine learning algorithms can be validated.

\section{Measuring Missed Emergency Department Acute Myocardial Infarction Diagnoses Using the SPADE Method}

\author{
A. L. Sharp ${ }^{1}$, A. Baecker ${ }^{1}$, N. Nassery², S. Park ${ }^{1}$, A. Hassoon², M. Lee ${ }^{3}$, S. Peterson ${ }^{4}$, S. Pitts ${ }^{4}$ and D. Newman-Toker ${ }^{5}$ \\ ${ }^{1}$ Kaiser Permanente Southern California, Department of Research and Evaluation, Pasadena, CA. \\ ${ }_{2}^{2} J o h n s$ Hopkins University School of Medicine, Department of Internal Medicine, Baltimore, MD. \\ ${ }^{3}$ Kaiser Permanente Southern California, Los Angeles Medical Center, Division of Cardiology, Los Angeles, CA. \\ ${ }_{4}^{4}$ Johns Hopkins University School of Medicine, Department of Emergency Medicine, Baltimore, MD. \\ ${ }^{5}$ Johns Hopkins University School of Medicine, Department of Neurology, Baltimore, MD.
}

Background: Diagnostic error is a serious public health problem; standardizing and reporting hospital and health system performance remains elusive. Our study aims to identify missed acute myocardial infarction (AMI) diagnoses using the symptom-disease pair analysis of diagnostic error (SPADE) method.

Methods: We analyzed electronic health records and administrative claims for adult members of Kaiser Permanente Southern California between 1/1/2009-12/1/2017. We first identified all AMI hospital admissions and "looked back" 30-days for any preceding treat-and-release Emergency Department (ED) visits. The top primary discharge diagnoses for ED visits prior to AMI were categorized and diagnoses with the highest prevalence and observed to expected (O/E) ratio were identified. Next, we identified all ED visits with the look-back symptoms and "looked forward" 30-days to identify potentially missed AMI diagnoses.

Results: There were 44,473 initial AMI hospitalizations during the study period. The most common treat-and-release ED visits in the preceding 30-days were for 'nonspecific chest pain' and 'dyspnea' with O/E ratios of 2.54 (95\% CI 2.34-2.76) and 3.70 (95\% CI 3.13-4.36) respectively. This represented 574 probable missed/delayed AMI diagnoses (1.3\%, 95\% CI 1.2\%-1.4\%). Looking forward, 325,088 chest pain and dyspnea ED 
encounters lead to 508 (0.2\%, 95\% CI 0.1\%-0.2\%) missed/delayed AMI diagnoses. The misdiagnosis rate was highest in the 7 days after the ED encounter and returned to a baseline rate by 30 days (Figure 1).

Conclusion: The SPADE method provides a standardized approach to identify missed AMI diagnoses using administrative data. A "look back" missed/delayed AMI rate of $\sim 1.3 \%$ corresponds to $>10,000$ US ED missed coronary events each year. Low rates of "look forward" missed/ delayed AMI diagnoses (0.2\%) may require health systems identify higher-risk sub-cohorts (demographic/clinical) to prevent long-term morbidity and mortality.

A

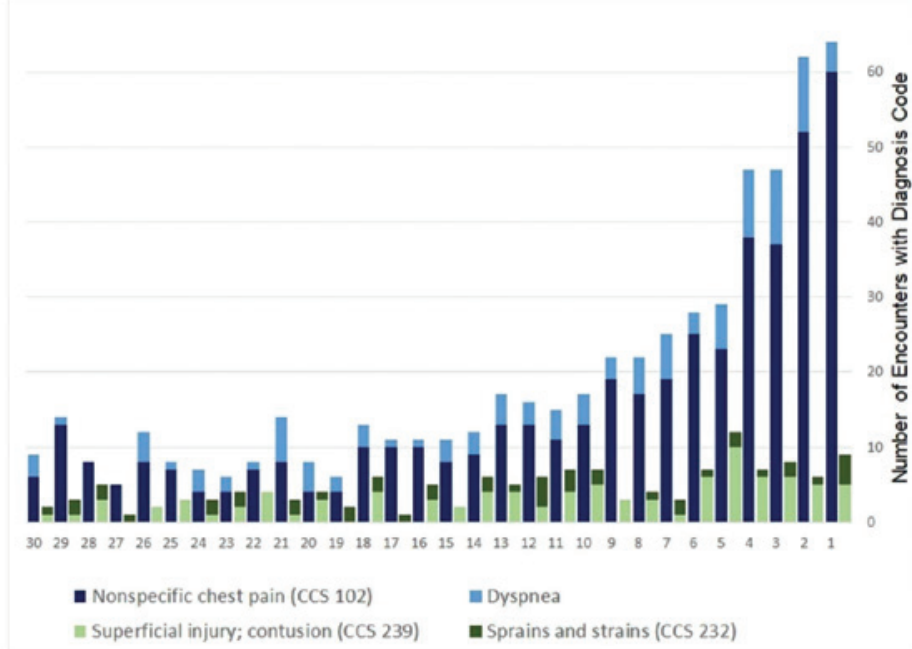

B

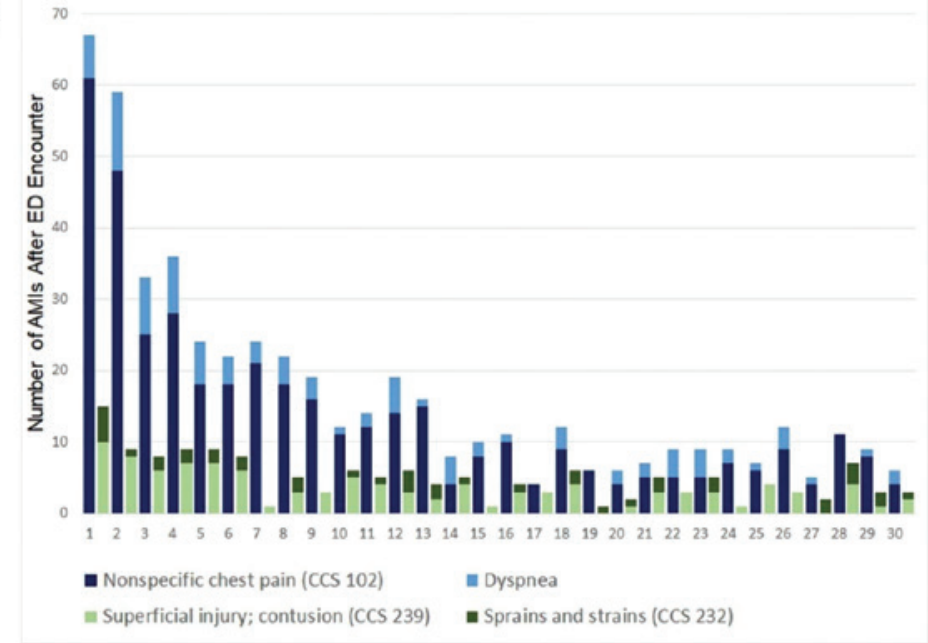

Figure 1 Daily counts of likely missed acute myocardial infarctions (AMI) using a look back (A) and a look forward (B) approach. Figure A represents ED diagnoses 1 to 30 days before an AMI hospitalization. Figure B represents an AMI hospitalization 1 to 30 days after an ED visit. Strains and sprains and superficial injuries are reported as a control group for comparison in both figures

\section{Identification of Clinical Phenotypes Among Patients with Misdiagnosed COPD Using Latent Class Analysis}

L. J. Spece ${ }^{1,2}$, P. J. Rise'2, L. M. Catucci ${ }^{3}$, L. M. Donovan ${ }^{1,2}$, P. Bhatraju' ${ }^{2}$, L. C. Feemster ${ }^{1,2}$ and D. H. $A u^{1,2}$

${ }^{1}$ Health Services Research and Development, VA Puget Sound, Seattle, WA

${ }^{2}$ University of Washington, Seattle, WA

${ }^{3}$ Health Services Research and Development, Office of Research and Development, VA Central Office, Washington, DC

Background: Chronic obstructive pulmonary disease (COPD) is the most common respiratory condition in the US with 24 million Americans affected, yet up to $50 \%$ do not have the disease when diagnostic spirometry is performed. In this context, COPD is a model to study the 
recalcitrant issue of diagnostic error. Patients with misdiagnosed COPD have a heterogeneous mix of comorbidities that may contribute to diagnostic uncertainty. Identification of clinical phenotypes within this misdiagnosed population will allow for precision in diagnosis and therapeutic delivery.

Methods: To identify phenotypes of misdiagnosed COPD, we used cross-sectional data from the COPD Outcomes-Based Network for Clinical Effectiveness and Research Translation (CONCERT) multi-center registry. COPD was defined by spirometry using the GOLD recommendation for airflow obstruction (FEV1/FVC $<0.70$ ). We applied latent class analyses to identify phenotypes. We utilized 12 clinical variables selected a priori based on literature supporting their role. Posterior probabilities were used to determine phenotype frequency, differences in patient outcomes, and proportions of COPD-directed treatments.

Results: Of 1,096 patients labeled with COPD, 43.2\% ( $\mathrm{n}=474)$ were misdiagnosed with COPD. A three-phenotype model had the best fit. The 3 phenotypes reported dyspnea on the medical research council (MRC) scale (1.6 alpha versus 0.9 beta versus 1.2 delta). Of the 3, the alpha phenotype was the most common $(n=575 ; 50.9 \%)$ and included patients with greater proportions of comorbidity, including anxiety $(79.9 \%)$, depression (66.1\%), congestive heart failure (24.1\%), and arthritis (46.2\%). The beta phenotype $(n=147 ; 13.4 \%)$ has the greatest proportion of obesity (56.9\%), never smokers (39.5\%), women (53.3\%), and non-white race (48.1\%). The delta phenotype $(\mathrm{n}=374 ; 34.1 \%)$ had the greatest probability of correct COPD diagnosis and had the lowest proportions of comorbidities, obesity (26.9\%), and never smokers (6.7\%) (Figure 1). The misdiagnosed alpha phenotype received similar treatments to the correctly diagnosed delta phenotype with inhaled corticosteroids (ICS) medications (14.6\% alpha versus 16.6\% delta) and combination long-acting bronchodilator/ICS inhalers (22.1\% alpha versus $26.7 \%$ delta). The beta phenotype received the fewest medications (8.8\% ICS; 16.3\% LABA/ICS). Exacerbation-like events were greatest among the alpha phenotype (12.5\%).

Conclusion: This analysis identified two clinically distinct groups at risk of COPD misdiagnosis with different predictors, treatments and outcomes. Interventions to correct diagnostic error in COPD may need to incorporate these phenotypes and tailor treatment approaches for symptoms that overlap with, but are not attributable to, COPD.

Figure 1. Chord diagram showing clinical variables by phenotype

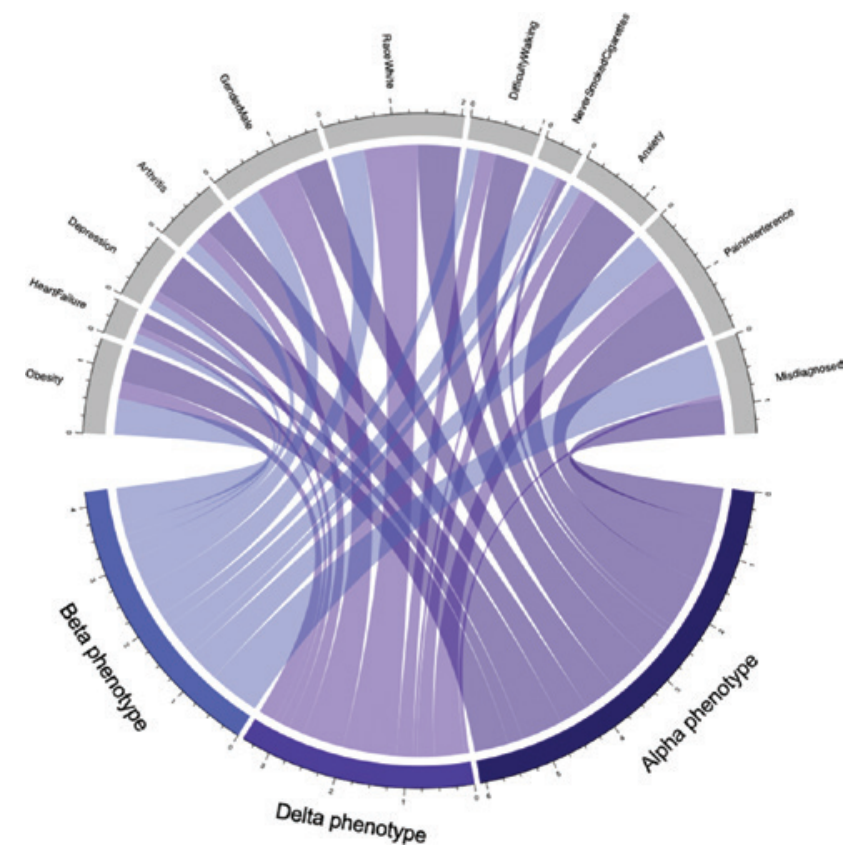

\title{
Thinking Fast or Slow, That's the Question? Are Cognitive Biases Related to Fast Diagnostic Reasoning?
}

\author{
L. Staal $^{1}$, J. Alsma 2 , S. Mamede ${ }^{1}$, A. Olsen ${ }^{3}$, M. Plesac ${ }^{3}$, M. Sundberg ${ }^{3}$, M.A. Frens ${ }^{4}$, H.G. Schmidt ${ }^{1}$, W.W. van den Broek ${ }^{1}$ and L. Zwaan ${ }^{1}$ \\ ${ }^{1}$ Erasmus Medical Center Rotterdam, Institute of Medical Education Research Rotterdam, the Netherlands \\ ${ }^{2}$ Erasmus Medical Center Rotterdam, Department of Internal Medicine, the Netherlands \\ ${ }^{3}$ University of Minnesota, Division of General Internal Medicine, U.S.A. \\ ${ }^{4}$ Erasmus Medical Center Rotterdam, Department of Neuroscience, the Netherlands
}

Background: Diagnostic errors have primarily been attributed to cognitive biases, which are thought to arise due to too selective and too fast diagnostic reasoning. Therefore, to prevent cognitive bias, slowing down the diagnostic reasoning process and reconsidering diagnoses is recommended with the aim to 'debias' physicians. However, whether a cognitive bias occurred, is determined in hindsight when we know the diagnosis was incorrect and therefore taking more time seems a logical solution. This multi-institutional study challenges the notion that 
diagnostic errors caused by cognitive biases arise when patients are diagnosed too quickly. Specifically, we will test whether diagnostic errors, particularly those due to cognitive biases, are diagnosed faster than correct diagnoses.

Methods: Currently, 95 internal medicine residents have participated. The experiment consists of two phases. In phase 1 we induced availability bias. The residents were randomly allocated to evaluate the diagnosis provided to cases of either set 1 or set 2 . Subsequently, in phase 2, all residents diagnosed new cases, half of which resembled cases from set 1 and half of which resembled cases from set 2. We aimed to replicate Mamede et al. (2010) and expected that in phase 2 residents would be biased towards the cases they had seen in phase 1 . We determined whether diagnoses were correct. If diagnoses were incorrect we determined whether this was due to a cognitive bias. We then compared the time to diagnose for the diagnostic errors due to cognitive biases, and the correct diagnoses. Data collection is still ongoing.

Results: Preliminary results show that average time to diagnose (in seconds) in the diagnostic phase was faster for correct diagnoses ( $M=109$, $\mathrm{SD}=33)$ than for all incorrect diagnoses $(M=140, S D=46), p<0.001, d=0.77$. Furthermore, availability bias was found in 20 cases diagnosed by the residents. To correct for the case residents were biased towards, time to diagnose was examined separately for both case sets. Time to diagnose did not differ between correct diagnoses (set1: $M=119, S D=53$; set2: $M=122, S D=49$ ) and incorrect diagnoses due to bias (set1: $\mathrm{M}=88, \mathrm{SD}=38$; set2: $\mathrm{M}=102, \mathrm{SD}=29)$ for set $1(\mathrm{p}=-.906)$ or set $2(\mathrm{p}=.197)$.

Conclusion: The results indicate that fast diagnostic reasoning is not only related to cognitive bias: fast reasoning can result in both correct and incorrect diagnoses. These findings contribute to our understanding of the causes of diagnostic errors and implicate that slowing down the reasoning process may not be an efficient intervention for improving diagnostic performance.

\title{
Diagnostic Error and Neuro-Ophthalmology: A Critical Issue
}

\author{
L. Stunkel, N. J. Newman and V. Biousse \\ Emory Eye Center, Emory University School of Medicine, Atlanta, GA, USA.
}

Background: Despite medical advances, studies show alarmingly high rates of diagnostic error (up to 15\% in the USA). Because diagnosis of neuro-ophthalmologic conditions requires a particularly resource-intensive and analytic approach, these complex conditions are particularly vulnerable to diagnostic errors. As a result, neuro-ophthalmology is uniquely positioned to improve our understanding of diagnostic error. This review summarizes the existing literature on diagnostic error relevant to neuro-ophthalmology practice.

Methods: PubMed search (English language) of relevant studies yielded 46 results on 05/20/2019. Studies that focused on the usefulness of a single test or intervention were excluded. Additional studies identified through hand search were included. A total of 15 studies involving more than 270,000 patients were included in this review.

Results: Recent studies on diagnostic problems directly relevant to neuro-ophthalmology include studies of misdiagnosis of posterior communicating artery aneurysms, idiopathic intracranial hypertension (IIH), optic neuritis, optic nerve sheath meningiomas (ONSM), and other neuro-ophthalmologic conditions that underwent inappropriate neuro-imaging. These studies identified misdiagnosis rates $>60-70 \%$ prior to neuro-ophthalmology referral. Major causes of diagnostic error in these conditions included cognitive failures such as failure to gather appropriate history, pre-established biases, and failure to generate an appropriately broad differential diagnosis, and pitfalls with ancillary tests, including failure to provide appropriate information to neuroradiology or lack of access to neuroradiology interpretation of images. Prior to evaluation by a neuro-ophthalmologist, many patients had unnecessary tests and treatments, including lumbar puncture in 16-80\%, brain MRI in $16-34 \%$, and inappropriate treatment in 11-76\%. Accurate diagnosis was delayed in the $10 \%$ of patients misdiagnosed with IIH. Misdiagnosis contributed to poor visual outcome in 64\% of patients in whom a diagnosis of ONSM was delayed.

Conclusion: Diagnostic error of neuro-ophthalmologic conditions leads to delay in diagnosis and unnecessary, costly and potentially harmful diagnostic tests and treatments. Better access to neuro-ophthalmologic evaluation has the potential to decrease inappropriate utilization of diagnostic tests and treatments and medical harm, and improve patient outcomes.

\section{Malpractice Claims Related to Diagnostic Errors in Japan}

T. Watari

Shimane University Hospital, Postgraduate clinical training center

Background: Previous research in the US has shown that diagnostic errors are a major cause of medical lawsuits. However, little is known about how much a diagnostic error contributes to medical malpractice claims in Japan. Therefore, we conducted a retrospective study on the background and effects of diagnostic errors using the largest internet database of claims in Japan.

Methods: The largest malpractice claims database in Japan (Westlaw Japan K.K) was used to identify diagnostic error-related and non-related malpractice claims. We excluded the duplicated and non-medical claims including intentional crimes, robbery, money troubles, and veterinary claims, using the exclusion criteria. We collected the following data: age and sex of the patient, specialty of the doctor, place or clinical setting, hospital size, initial and final diagnosis, acceptance amount, death, presence of sequelae, etc. All amounts were adjusted with the consumer price index. Results: In the 1972 medical malpractice claims analyzed, the median age of patients was 33.5 years (IQR: 10-54), and 53.7\% were male. The medical outcomes included deaths in 939 cases (53.3\%). In total, 725 of 1972 malpractice claims (37.4\%) were diagnostic error-related cases. 
On comparing the diagnostic error-related $(n=709)$ and non-related $(n=1093)$ cases, the mortality rates were found to be $62.3 \%$ and $45.5 \%$, respectively ( $p$-value $<0.001$ ); cases of internal medicine were significantly higher in the former 30.5\% and 19.0\%, respectively ( $p$-value $<0.001$ ); small-size hospitals were higher in the former $23.3 \%$ and $17.3 \%$, respectively ( $p$-value $=0.002$ ); cases in general outpatient and emergency room were higher in the former $42.9 \%$ and $15.8 \%$, respectively ( $p$-value $<0.001$ ); cases during the night shift $17.5 \%$ was higher than case of day shift 12.0\% ( $\mathrm{p}$-value $<0.001$ ). Additionally, the acceptance rates of diagnostic error-related and non-related claims were $63.6 \%$ and $46.0 \%$, respectively ( $\mathrm{p}$-value $<0.001$ ), and the median claim payments of both groups were $\$ 231181$ (IQR $\$ 50150-\$ 484546$ ) and $\$ 136,363$ (IQR \$30554-\$370 000). This is the first study in Japan that focused on the negative impact of malpractice claims relating to diagnostic errors. In Japan, recently, attention has been directed to diagnostic errors. The diagnostic error-induced malpractice claims were as high as $37.4 \%$, which was almost similar to prior reports in the US.

Conclusion: Based on the analysis of the malpractice claims database in Japan, diagnostic errors were the main causes of medical claims and contribute negatively to many aspects.

\begin{tabular}{|c|c|c|c|c|c|c|}
\hline & & \multirow{2}{*}{\multicolumn{2}{|c|}{$\begin{array}{l}\text { Diagnostic error-related claims } \\
\qquad(n=709)\end{array}$}} & \multirow{2}{*}{\multicolumn{2}{|c|}{$\begin{array}{l}\text { Non diagnostic error-related claims } \\
\qquad(n=1093)\end{array}$}} & P-value \\
\hline & & & & & & \\
\hline \multicolumn{2}{|c|}{ Patient gender ( male \%) } & \multicolumn{2}{|c|}{$56.1 \%$} & \multicolumn{2}{|c|}{$53.0 \%$} & 0.211 \\
\hline \multicolumn{2}{|c|}{ Patient age } & \multicolumn{2}{|c|}{35 (IQR16-55) } & \multicolumn{2}{|c|}{32 (IQR 4-53) } & 0.008 \\
\hline \multicolumn{2}{|c|}{ Adjusted total billing amount } & \multicolumn{2}{|c|}{$440,000$ (IQR $185,454-775.455)$} & \multicolumn{2}{|c|}{$350,909$ (IQR $107,272-660,000)$} & $<0.001$ \\
\hline \multicolumn{2}{|c|}{ Acceptance } & \multicolumn{2}{|c|}{$451 / 709(63.6 \%)$} & \multicolumn{2}{|c|}{$503 / 1093(46 \%)$} & $<0.001$ \\
\hline \multicolumn{2}{|c|}{ Adjusted total accepted amount } & \multicolumn{2}{|c|}{231,181 (IQR50,150-484,546) } & \multicolumn{2}{|c|}{$136,363$ (IQR $305,54-370,000)$} & $<0.001$ \\
\hline \multicolumn{2}{|c|}{ Duration of claim case } & \multicolumn{2}{|c|}{$7.48( \pm 3.68)$} & \multicolumn{2}{|c|}{$7.99( \pm 4.06)$} & 0.0054 \\
\hline \multicolumn{7}{|c|}{ Clinical Feileds of malpractice claims } \\
\hline & Internal medicine & 216 & $30.5 \%$ & 208 & $19.0 \%$ & $<0.001$ \\
\hline & Obstetrics and Gynecology & 114 & $16.1 \%$ & 221 & $20.2 \%$ & 0.027 \\
\hline & Surgery & 150 & $21.2 \%$ & 189 & $17.3 \%$ & 0.04 \\
\hline & Orthopedics & 45 & $6.3 \%$ & 117 & $10.7 \%$ & 0.002 \\
\hline \multicolumn{7}{|c|}{ Outcome } \\
\hline & Deaths & 442 & $62.3 \%$ & 497 & $45.5 \%$ & $<0.001$ \\
\hline & Sequelae & 219 & $30.9 \%$ & 475 & $43.5 \%$ & $<0.001$ \\
\hline \multicolumn{7}{|c|}{ 1st Diagnosis and rank } \\
\hline 1 & Traumatic injury & 62 & $8.7 \%$ & 94 & $8.6 \%$ & 0.014 \\
\hline 2 & Malignant neoplasm & 65 & $9.2 \%$ & 85 & $7.8 \%$ & 0.291 \\
\hline 3 & Neonatal complications & 29 & $4.1 \%$ & 87 & $8.0 \%$ & 0.001 \\
\hline 4 & Procedure and postoperative complications & 14 & $2.0 \%$ & 65 & $5.9 \%$ & $<0.001$ \\
\hline 5 & Respiratory tract infection & 50 & $7.1 \%$ & 27 & $2.5 \%$ & $<0.001$ \\
\hline 6 & Ischemic heart disease & 32 & $4.5 \%$ & 40 & $3.7 \%$ & 0.366 \\
\hline 7 & Systematic infectious disease & 27 & $3.8 \%$ & 36 & $3.3 \%$ & 0.561 \\
\hline 8 & Mental disorder & 12 & $1.7 \%$ & 45 & $4.1 \%$ & 0.004 \\
\hline 9 & Non-bleeding digestive tract disease & 37 & $5.2 \%$ & 15 & $1.4 \%$ & $<0.001$ \\
\hline 10 & No abnormality & 40 & $5.6 \%$ & 8 & $0.7 \%$ & $<0.001$ \\
\hline
\end{tabular}

\title{
EpiFinder: Helping to Improve Epilepsy Diagnosis
}

\author{
$\underline{\text { R. Yao }}^{1}$, L. Csernak ${ }^{1}$, E. Salinas ${ }^{1}$, E. Okazaki² and J. Sirven ${ }^{2}$ \\ ${ }^{1}$ Arizona State University \\ ${ }^{2}$ Mayo Clinic Arizona
}

Background: In the 2012 Institute of Medicine (IOM) Epilepsy Across the Spectrum report, epilepsy was identified as a major neurological and public health problem that affects 1 in 26 individuals over their lifetime, 3.4 million people in the U.S., and 65 million people worldwide. Epilepsy is misdiagnosed upon initial screening in $35-40 \%$ of all cases often resulting in delays to diagnosis and therapy. The IOM report identified the inability of physicians to make or agree upon a diagnosis as a major cause for inadequate management and created recommendations for the data collection, ascertainment, identification and education of seizures and epilepsy syndromes (1). To address this diagnostic and therapeutic gap, we tested a novel clinical decision support tool, EpiFinder. This application takes key words from a patient's history and using a heuristic algorithm, dynamically produces a list of differential diagnoses. We evaluated the utility and accuracy of EpiFinder in providing an accurate diagnosis in an adult cohort.

Methods: Patients were prospectively identified upon admission to the epilepsy monitoring unit (EMU) at Mayo Clinic Arizona. A series of 50 patients admitted for spell classification consented participation and were asked to describe their events in detail. The terms and phrases used by the patients were input into the EpiFinder application. The application generated a list of potential differential diagnoses if a seizure disorder was under consideration. If the application did not identify the existence of such a disorder, it would indicate that the information provided was insufficient. The EpiFinder-generated results were then compared to the diagnosis that resulted from the patient's EMU stay. Results were considered positive if the EpiFinder application produced a differential diagnosis consistent with an epilepsy syndrome. 
Results: A total of 50 patients admitted to the EMU for scalp EEG monitoring over a five month period from January through May 2017 were included. During that time, 26 patients were diagnosed with a seizure disorder, 22 patients were given a diagnosis of non-epileptic spells and two patients were found to have a parasomnia. The EpiFinder application was able to correctly identify the presence of a seizure disorder versus non-seizure etiology in 43 patients or $86 \%$ of the time with sensitivity of $89.5 \%$ (95\% CI $77.3-96.5$ ) and specificity of $87.5 \%$ (95\% CI 67.6-97.3).

Conclusions: EpiFinder is a clinical decision support tool that can be used by providers to help identify epilepsy. This resource could streamline epilepsy diagnosis and management.

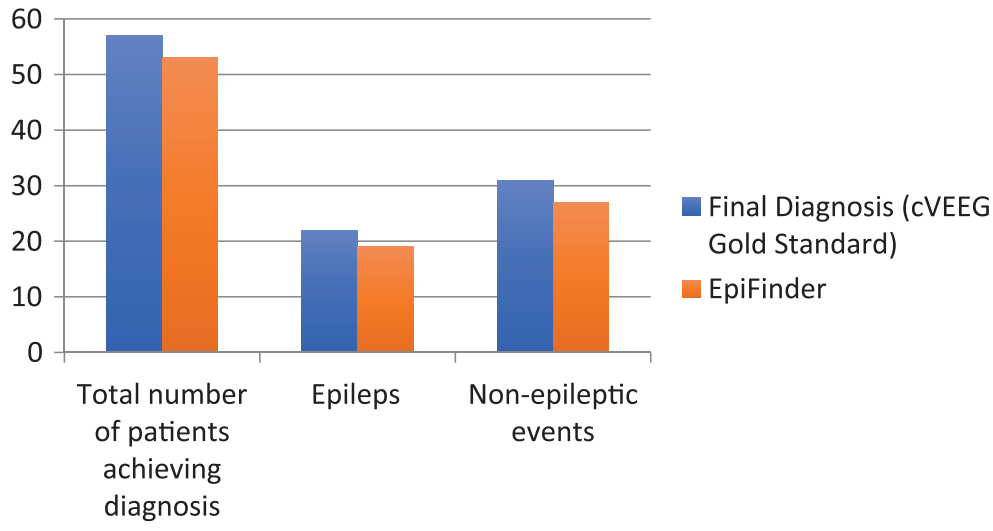

(1) England MJ, Liverman CT, Schultz AM, Strawbridge LM. Epilepsy across the spectrum: Promoting health and understanding.: A summary of the Institute of Medicine report. Epilepsy \& Behavior 2012;25(2):266-276.

\title{
Top Contributors to Missed Test Results in the VA Health Care System
}

\author{
A. Zimolzak ${ }^{1,2}$, U. Shahid ${ }^{1,2}$, S. Memon ${ }^{1,2}$, T. Giardina ${ }^{1,2}$, D. Murphy ${ }^{1,2}$, L. Zubkoff ${ }^{3,4}$, H. Singh $^{1,2}$ and U. Mushtaq ${ }^{1,2}$ \\ ${ }^{1}$ Michael E. DeBakey VA Medical Center. \\ ${ }^{2}$ Baylor College of Medicine. \\ ${ }^{3}$ White River Junction VA Medical Center. \\ ${ }^{4}$ Dartmouth Geisel School of Medicine.
}

Background: Lack of timely follow-up of test results ("missed test results") is relatively frequent in medical practice. Systematic reviews have shown that electronic health record (EHR) systems can increase test results follow-up, but the evidence is weak. As part of an effort to assist EHR-enabled organizations in the prevention of missed test results, this study aimed to identify common high-priority risk areas and contributing factors related to missed test results in a national sample of VA health care facilities.

Methods: We developed a semi-structured interview guide based on existing literature. The guide included 42 questions that addressed patient, care-team, and systems-related factors involved in reliable test results follow-up. We conducted interviews with various stakeholders involved in diagnostic test results communication at geographically diverse and variable sized health care facilities. We recorded and transcribed interviews and used content analysis to analyze data and generate salient themes that increased risk of missed test results.

Results: Participants $(\mathrm{n}=22)$ from 8 out of 12 planned sites have been interviewed thus far and included: clinicians $(n=7,32 \%)$, facility leader$\operatorname{ship}(n=5,23 \%)$, lab professionals $(n=4,18 \%)$, radiology professionals $(n=1,5 \%)$, nursing $(n=2,9 \%)$, and patient safety professionals $(n=2$, 9\%). Upon content analysis, we identified the following eight risk areas: the EHR and related tracking systems; trainees/residents; referral coordination outside the initiating medical center; lack of updated patient and provider contact information; incidental findings on imaging (such as suspicion of cancer); lack of clarity regarding the clinician responsible for lab follow up; inconsistent methods of patient notification and related tracking; and use of surrogate/back-up clinicians. The three most commonly cited risk areas included: EHR notifications related to test results are not managed well by trainees or can be lost because trainees rotate frequently (mentioned by 14 unique participants), secondary radiology findings often fall through the cracks because no one "owns" them (14 participants), and there is no fail-safe method of tracking abnormal results via the EHR (12 participants).

Conclusion: Analysis identified three main risk areas that contribute to missed test results: problems in management of EHR notifications sent to trainees, lapses in management of secondary/incidental radiology findings, and under-developed EHR-based systems to track test results. Findings are guiding development and implementation of multi-faceted interventions to target these risk areas. 


\title{
Clinical Vignettes
}

\section{The Dark Effusion: A Diagnosis Hidden by Framing Bias}

\author{
V. Barksdale ${ }^{1}$ and M. Duncan ${ }^{2}$ \\ ${ }^{1}$ University of Colorado School of Medicine, Aurora, CO \\ ${ }^{2}$ University of Colorado Hospital, Aurora, CO
}

Case information: A 50-year-old female with history of anorexia/bulimia, alcohol use disorder and chronic pancreatitis presented to the emergency department overnight with atypical chest pain. Acute coronary syndrome workup was negative, lipase was $480 \mathrm{U} / \mathrm{L}$, and abdominal ultrasound was unremarkable. She was given a diagnosis of acute pancreatitis and failure to thrive, and admitted for IV fluids, physical therapy and rehabilitation placement.

During morning rounds, the patient complained of persistent left-sided pleuritic chest pain and a chest x-ray revealed a large left pleural effusion. Diagnostic thoracentesis showed a black aspirate that was exudative with an amylase level of 10,915 U/L. She underwent ERCP, with a final diagnosis of pancreaticopleural fistula.

Discussion: A black pleural effusion is a rare and striking feature that quickly narrows the differential diagnosis. Some important causes of black pleural effusions are malignancy (including metastatic melanoma), fungal infections, and pancreaticopleural fistulae. A pancreaticopleural fistula is a rare complication of pancreatitis and presents as a large, often left-sided pleural effusion with amylase-rich pleural fluid. During handoff, the patient's one-liner highlighted psychiatric illness and substance misuse, which promoted misleading framing and visceral bias. When framed this way, the team anchored on acute pancreatitis despite the fact that she had no characteristic symptoms or radiologic evidence, and thus did not meet diagnostic criteria. Providers manifest visceral bias as countertransference when they perceive a patient as difficult, and is often related to mental illness or substance use. The result is suboptimal care including dismissal of complaints. When the team reframed the presentation around her chief complaint, they discerned a black pleural effusion and brought to light her true diagnosis of pancreaticopleural effusion.

\section{Renal Failure and a Clavicular Mass: Don’t Cut Yourself on Occam's Razor}

\author{
$\underline{\text { S. Blaser }}{ }^{1}$, P. Cocks ${ }^{2}$ and D. Kudlowitz ${ }^{3}$
}

${ }^{1}$ Department of Medicine, NYU School of Medicine

${ }^{2}$ Department of Medicine, Division of General Internal Medicine and Clinical Innovation

${ }^{3}$ Department of Medicine, NYU School of Medicine

Case Summary: A 66-year-old woman with hypertension presented to the hospital one day after arrival to New York City from Guinea with chronic daily vomiting, unintentional weight loss, progressive shoulder pain, and a subacute pruritic rash.

On exam, patient was hypertensive, had limited range of motion in her right shoulder, scaling plaques over legs and trunk, and asterixis. Labs were notable for a creatinine of 15 with normocytic anemia. Calcium was normal. The patient was admitted to hospital for acute renal failure and further workup.

Diagnoses: Primary end stage renal disease of unknown etiology; and follicular thyroid carcinoma with metastases to the clavicle and lungs with paraneoplastic rash.

Discussion: This case highlights the diagnostic principles of Occam's razor, Hickam's dictum, and Crabtree's bludgeon. The initial differential diagnosis and workup proceeded with the expectation of a unifying diagnosis to explain the wide constellation of presenting symptoms. But after common systemic unifying diagnoses, including multiple myeloma and other infiltrative processes, were ruled out, it became evident that two processes were at play. The first was end stage renal failure, likely long-standing given imaging findings suggestive of chronicity. The second, a common malignancy with uncommon metastases. Perhaps an absence of regular interactions with the healthcare system prior to presentation increases like the positive predictive value of Hickam's dictum. Additionally, common diseases with uncommon presentations are still more common than zebras. Lessons from this case allows us to expand our thinking beyond the dogma of any one diagnostic principle, to avoid type I thinking and help us to direct our diagnostic reasoning. 


\title{
Anchors Away: Drifting on a Sea of Assumptions
}

\author{
T. L. Brazile ${ }^{1}$, P. Patton ${ }^{2}$ and T. Nikiforova ${ }^{1}$ \\ ${ }^{1}$ Internal Medicine, University of Pittsburgh Medical Center \\ ${ }^{2}$ University of Pittsburgh School of Medicine
}

Case: A 60 yo female nurse presented to her cardiologist with one year of progressive exertional dyspnea with chest pressure, fatigue, nausea and vomiting. Her exam was unremarkable. An EKG showed chronic LBBB. She underwent a stress cardiac MRI which revealed normal EF and absence of ischemic or infiltrative disease. She was referred to pulmonology. PFTs and CXR were normal. An ABG showed normal oxygenation and incidentally revealed a hemoglobin of 5 . She was subsequently hospitalized. Her labs were consistent with profound iron deficiency anemia (IDA). Upon further questioning, the patient reported a history of a subtotal colectomy and 8-months of back pain treated with ibuprofen. EGD and sigmoidoscopy were concerning for an occult bleed from her ileosigmoid anastomosis.

Discussion: Blood loss is the most common cause of IDA worldwide and can be difficult to recognize if the source is not obvious. Exertional dyspnea is a frequent symptom of anemia, and workup should begin with a CBC, SpO2, and EKG. In this case, diagnosis and treatment were delayed as cardiologists anchored on the patient's chest pain and LBBB and pursued a focused cardiac workup despite a normal exam. Availability bias also affected this case as specialists likely overestimated the likelihood of cardiac or pulmonary causes for this patient's dyspnea based on their experiences with those diseases. Obtaining a complete medical history, updating the medication list, and starting with a broad differential may have prevented this costly hospitalization. Structured data acquisition may reduce the use of heuristics in developing a differential, while deliberate decoupling and mindfulness engage analytical thought processes that can improve diagnostic accuracy.

\section{Blinded by the Pathway: Not All Troponins Are Myocardial Infarctions}

D. M. Cantu and J. N. Lessing

University of Colorado Internal Medicine

Case Information: Patient is a middle-aged man with alcohol use disorder, hypertension, and mitral valve prolapse who presents to the emergency department (ED) with diffuse symptoms including lightheadedness and pre-syncope without discrete chest pain. His clinical picture is complicated by alcohol withdrawal symptoms. Initial troponin is 0.2 and electrocardiogram shows possible ST segment changes, which are deemed consistent with repolarization abnormality. However, based on the hospital's ACS Evaluation Pathway, he is admitted to the cardiology service.

Repeat troponins persist at 0.2. Later in the hospitalization, in the absence of typical chest pain, the team seeks other causes of troponin elevation. An elevated dimer and then CT-PE confirms a large saddle pulmonary embolism with evidence of right heart strain. Anticoagulation is started and he later discharges home.

Discussion: This case demonstrates just one cause of elevated troponin from many examples of non-MI disease states (table 1 adapted from American College of Cardiology). ${ }^{1}$ Keeping a broad differential particularly for troponins without a distinctive rise or fall pattern is important for considering non-MI causes of troponin elevations. A study of almost 70,000 admitted ED patients found nearly half had troponins checked and of those with elevated troponins $43 \%$ had causes other than ACS (Waxman 2006). ${ }^{2}$ Clinical pathways standardize medical practice and facilitate efficient delivery of care but should not be followed blindly. Admission diagnosis such as "Rule out ACS" or disposition decisions based primarily on abnormal lab values can preclude broad thought, result in premature closure, and delay accurate diagnosis.

\section{References:}

${ }^{1} J a n u z z i$, James L. Causes of Non ACS Related Troponin Elevations. [Internet]. 2010 September 8. [cited 2019 June 5]. Available from: https:// www.acc.org/latest-in cardiology/articles/2014/07/18/13/16/causes-of-non-acs-related-troponin-elevations ${ }^{2}$ Waxman, D.A., et al., A model for troponin I as a quantitative predictor of in-hospital mortality. J Am Coll Cardiol 2006. 48(9): p. 1755-62.

\begin{tabular}{|c|c|c|c|c|c|c|c|}
\hline Cardiovascular & Myocardial Injury & Pulmonary & Gastrointestinal & Musculoskeletal & Neurological & Renal & Infectious \\
\hline Arrhythmia & Blunt Chest Trauma & Acute PE & Severe GI Bleeding & Rhabdomyolysis & Acute Stroke & CKD & Endocarditis \\
\hline \multirow{2}{*}{$\begin{array}{l}\text { Acute Aortic } \\
\text { Dissection }\end{array}$} & Cardiac Surgeries & ARDS & & & Head Trauma & & Myocarditis \\
\hline & Chemotherapy & & & & & & Sepsis/SIRS \\
\hline $\begin{array}{l}\text { Apical Ballooning } \\
\text { Syndrome }\end{array}$ & EP Procedures & & & & & & \\
\hline \multicolumn{8}{|l|}{ Heart Failure } \\
\hline \multirow{2}{*}{\multicolumn{8}{|c|}{$\begin{array}{l}\text { Severe } \\
\text { Hypertension }\end{array}$}} \\
\hline & & & & & & & \\
\hline \multicolumn{8}{|l|}{ Severe } \\
\hline Hypotension & & & & & & & \\
\hline
\end{tabular}




\section{Diagnostic Challenges in Vulnerable Patient Populations}

C. Caviglia, J.N. Lessing and D. Klimpl

University of Colorado Hospital

Learning objectives: Recognize vulnerable patient presentations where diagnostic accuracy can be compromised by incomplete data and subsequent premature closure.

Case information: A 59-year-old Spanish-speaking woman presented with three days of right sided flank pain, oliguria and malaise. On presentation, history was limited by language barrier and physical exam was limited by super morbid obesity (BMI 45). Based on reported symptoms and bacteriuria, pyelonephritis was our presumptive diagnosis. The patient transiently improved on ceftriaxone, but on hospital day two developed encephalopathy, worsening leukocytosis, and acute renal failure. Liver function tests and procalcitonin were concerning for biliary obstruction and worsening bacterial infection, however encephalopathy and morbid obesity again limited meaningful history or physical exam. Both transabdominal ultrasound and MRCP were attempted but were unable to be performed secondary to the patient's size. On hospital day four, a CT scan showed acute cholecystitis as the actual diagnosis. Percutaneous cholecystostomy drain placement and broad-spectrum antibiotics led to eventual improvement.

Discussion: In a time where there is increasing emphasis on high value care, it is important to identify patient characteristics that could lead to incomplete data and subsequent medical errors. Patients who are cognitively impaired, chronically ill and/or disabled, economically disadvantaged, of minority populations, and non-English speaking are especially vulnerable, and might benefit from an expanded differential diagnosis on initial workup. In our patient, a language barrier combined with an unreliable physical exam should have prompted an earlier and expanded differential for other potential causes of right flank pain, and additional testing to confirm the diagnosis.

Broadening the diagnostic approach among patients who are identified as vulnerable based on circumstance and social determinants of health can lead to reduced health care disparities and improved patient outcomes.

\section{Occult Upper Airway Obstruction Masquerading as Aspiration}

\section{E. Deal, S. Hatfield and B. Giacomino}

Baylor College of Medicine

Learning objectives: Recognize the presence of upper airway obstruction. Remain vigilant of medical equipment in patients with altered mentation.

Case information: A 74-year-old woman with advanced Parkinson's and vascular dementia presented to our hospital with PEG-tube feeding intolerance and concern for aspiration. At baseline, she is bed-bound, non-verbal, does not recognize family and is fed by PEG-tube. According to family, PEG tube feeds had been appearing in her mouth for two days. CT chest imaging on admission showed left main-stem bronchial debris and bilateral lower lobe opacities concerning for aspiration; there was no upper airway obstruction. Repeat suctioning of the upper airways was performed via nasopharyngeal trumpet. Despite antibiotics and frequent suctioning, the patient continued to desaturate on supplemental oxygen. On day two of hospitalization, a rapid response was called for worsening hypoxia. The patient was saturating $76 \%$ on non-rebreather mask. The patient was in frank respiratory distress, but no stridor was appreciated. Intubation was initiated via video laryngoscopy, at which time a nasopharyngeal airway trumpet was noted to be obscuring the airway at the level of the vocal folds. The nasopharyngeal trumpet was removed via Magill forceps and the patient was subsequently intubated.

Case discussion: Upper airway obstruction is a known cause of respiratory failure in at-risk populations. In patients with altered mentation, the usual signs and symptoms of upper airway obstruction may be blunted. When encountering a case of acute respiratory failure, it is important to consider airway patency. Although it remains unclear as to how this patient developed airway obstruction, it is important to be aware of all medical equipment being utilized in patient care.

\section{Anchoring on an Unlikely Diagnosis: A Case of Primary Focal Segmental Glomerulosclerosis Misdiagnosed as Minimal Change Disease}

\section{Diab, S. Chang and K. Timmins}

Baylor College of Medicine

Objectives: Review a case of anchoring bias in the setting of biopsy sampling error Discuss how to limit anchoring bias during the diagnostic process

Case: A 61-year-old African-American man with recently diagnosed nephrotic syndrome presented to our hospital with two weeks of progressive generalized edema and dyspnea. Nine months prior he presented with identical symptoms and was found to have nephrotic range proteinuria. He underwent renal biopsy showing minimal change disease (MCD). He was discharged on diuretics and prednisone with initial improvement in swelling and proteinuria. He then began to develop generalized edema, prompting his return to the hospital and was found 
to have renal failure with nephrotic range proteinuria concerning for relapse of MCD. Prednisone was increased and aggressive diuresis was initiated. Despite several days of therapy there was no clinical improvement. Repeat renal biopsy was performed demonstrating Primary Focal Segmental Glomerulosclerosis (FSGS).

Discussion: Our case highlights anchoring bias, a cognitive bias in which clinicians rely too heavily on a piece of information in the diagnostic process and do not adjust when disconfirming evidence arises.

Our patient was initially diagnosed with MCD which is seen in $<15 \%$ of cases of nephrotic syndrome, whereas primary FSGS accounts for up to $80 \%$ of nephrotic syndrome in African-American adults. Additionally, his clinical course was atypical, as MCD is typically highly responsive to steroids while primary FSGS shows variable response. Sampling error accounted for the misdiagnosis as the initial biopsy sample had 18 glomeruli, while some studies suggest $>25$ glomeruli be present for confident diagnosis.

Our patient was managed as MCD for several days during his hospitalization before reevaluating with repeat biopsy, prolonging his stay unnecessarily. To avoid anchoring bias, it is important to correlate the diagnosis with the clinical picture and be cognizant of the limitations of tests in the diagnostic process.

\title{
Zoster Voiding Dysfunction
}

T. Fukushima, S. Masaji, T. Wakai, S. Constantine and N. Urara

Sapporo Tokushu-kai Hospital

The University of Michigan, Department of Family Medicine

Learning objectives: Rarity may be a barrier for aggressive treatment or referral to specialist regardless of typical presentation.

Case information: The patient is 81-year-old female with history of breast cancer status post mastectomy presented with lower abdominal pain and urinary retention and was admitted to our hospital. She started to have difficulty urinating and visited urology clinic and intermittent catheter was instructed. The rash was noted in sacral area, and Varicella zoster virus (VZV) infection was suspected but no treatment or referral were recommended. The rash started to spread and visited dermatology 2 days prior to hospitalization. Oral acyclovir was prescribed but the rash didn't improve. The urinary incontinence didn't improve and started to have lower abdominal pain, which brought her to the emergency department in Sapporo Tokushu-kai hospital. She had vesicles distributed in sacral, gluteal and pubic area, which were consistent with VZV infection. Based on the clinical presentation, Sacral herpes zoster associated with voiding dysfunction was suspected. High-dose acyclovir was initiated, and urinary incontinence and rash got better after 2 weeks of hospitalization. She was discharged to home without any complications.

Discussion: VZV in sacral lesion is found only $4 \%$ of cases, furthermore, it rarely causes voiding dysfunction. Generally, it responds well to antiviral treatment and improves as the rash disappears. This patient had urinary incontinence and visited the urology clinic, and she already had rash in sacral area, and VZV infection was suspected. However, anti-viral medicine was not initiated nor dermatology was referred. The rash and urinary incontinence didn't improve, followed by hospitalization for intravenous acyclovir. Since the incidence of this is rare, there is no evident data to support the timing of the initiation of anti-viral medicine may impact the outcomes. Antiviral medicine was not initiate initially likely due to insufficient knowledge to associate urinary incontinence and VZV.

\section{Re-examining the Diagnostic Criteria for Wilson Disease: A Case Report}

\author{
L. Hummel ${ }^{1}$, C. Carr², S. Biow ${ }^{3}$, T. Sauer ${ }^{4}$ and M. Maylin ${ }^{5}$ \\ ${ }^{1}$ Tulane University School of Medicine \\ ${ }^{2}$ Tulane University-Ochsner Clinic Foundation Program, Department of Neurosurgery, Tulane University Medical Center \\ ${ }^{3}$ Tulane University School of Medicine \\ ${ }^{4}$ Ophthalmic Consultants of Boston, Boston, MA \\ ${ }^{5}$ Department of Internal Medicine, Tulane University Medical Center
}

Learning Objectives: Wilson Disease is a genetic disorder of copper metabolism with subsequent accumulation in multiple organs, especially the liver, brain, and cornea. There are over 600 described mutations of the culprit ATP7B gene and countless phenotypes affecting the hepatic, ocular, and neuropsychiatric systems. As no single test has adequate sensitivity or specificity for Wilson Disease, proper diagnosis is challenging. Current diagnostic guidelines rely on a scoring algorithm from a series of biochemical and clinical tests that attempt to account for the myriad of possible manifestations.

Case Information: A 54-year old woman with history of hepatitis C, encephalopathy, borderline personality disorder, and hypothyroid presented with fever, chills, cough, and low back pain. She was found with endocarditis, bacteremia, liver mass, and osteomyelitis with noncompressive epidural abscess requiring prolonged inpatient stay. Investigations revealed history of acute-on-chronic dementia with poor performance on formal cognitive testing and a family history of early cirrhosis and a heterogeneous range of neuropsychiatric disorders with early cognitive decline. Brownish-yellow rings were seen around Descemet's membrane, suggestive of Kayser-Fleischer rings. It was considered that the patient had undiagnosed Wilson Disease. Testing showed low ceruloplasmin but normal 24-hour urinary copper excretion. As her condition improved with zinc, lactulose, rifaximin, IV antibiotics, and lumbar laminectomy with abscess drainage, no further workup was undertaken. 
Discussion: Our patient met many diagnostic criteria for Wilson Disease, including liver findings, ceruloplasmin level, neuropsychiatric symptoms, and Kayser-Fleischer rings, scoring a total of 5 points on the EASL scale, which is sufficient for diagnosis of Wilson disease. Nevertheless, considering the low sensitivities and specificities for many of these tests and our patient's comorbidities, we believe that she met diagnostic criteria for Wilson Disease without having the disease. This case suggests a need to critically re-examine the diagnostic criteria for Wilson Disease.

\title{
Barriers of Sense of Shame in Female Patients to Male Provider Delayed the Detection of Visible Advanced Cancers
}

\author{
K. Ishikawa, S. Masaji, T. Wakai, S. Constantine and N. Urara
}

Sapporo Tokushu-kai Hospital

The University of Michigan, Department of Family Medicine

Learning objective: Male providers need to be sensitive to female patients in taking sensitive information. However, it is essential for male providers to pursue it without hesitation if it is necessary.

\section{Case information:}

Case 1: The patient is 79-year-old female with history of hypertension presented with coxalgia. She has been regularly followed by our family medicine clinic for hypertension. On the exam, she was found to have distended abdomen. She noticed her abdomen had been distended over one year, but she thought she just gained weight and didn't want to address it because she felt ashamed of the weight gain. Bedside abdominal ultrasound revealed gigantic polycystic mass, which was confirmed as ovarian polycystic tumor (24X18cm) by CT and MRI. Gynecologist was referred and oophorectomy was performed, which revealed malignant ovarian tumor.

Case 2: The patient is 85-year-old female with history of hypertension and osteoporosis presented for regular follow-up. She has been regularly followed by our clinic. She was found to have unexplainable thrombocytosis, and bedside abdominal ultrasound was performed to rule out asplenia. However, the examiner noticed gauze was applied on the left breast. Further examination showed gigantic nodule with ulcer on the left breast. Based on further history, patient mentioned that she had had breast lump since 2 years ago, but she didn't address it because she didn't want her breast to be examined by male provider because she felt ashamed. She was referred to breast cancer center for further treatment.

Discussion: Barriers of sense of shame in the female patients to male providers were barriers for the visible advanced cancers to be identified earlier, before developing to advanced stages in these two cases. Both two patients had been regularly followed by our clinic and opportunities to be addressed have been missed in those encounters, due to faulty data gathering.

\section{Beyond the Big Three: Central Retinal Artery Occlusion and Digital Ischemia due to Underlying Eosinophilic Granulomatosis with Polyangiitis}

S. Jain, S. Hansen and J. Lessing

University of Colorado Internal Medicine

\section{Learning Objectives:}

1. To increase awareness of the multisystem manifestations of EGPA.

2. To encourage reflective pause and consideration of syndromic diseases when a patient presents with a constellation of symptoms.

Case Information: A 57-year-old man with asthma presented with sudden, vision-loss of his left eye, accompanied by pleuritic chest pain and severe burning extremity pain.

Exam showed complete left eye vision-loss, absent left afferent pupillary reflex, bilateral hand numbness, splinter hemorrhages and black finger discoloration. Funduscopic evaluation revealed extensive intravascular clots including central retinal artery occlusion. Absolute eosinophil count was 1200 (10.2\%), c-ANCA was 1:640 and Serine Protease-3 IgG was 290. Upper extremity arteriogram revealed bilateral radial and ulnar arterial occlusions and cardiac MRI showed myocardial inflammation. Prolonged treatment with methylprednisolone, cyclophosphamide and heparin led to normalization of inflammatory markers, vision improvement to light perception and reduced hand pain.

Discussion: EGPA is an uncommon disease with severe consequences if not treated promptly. Mortality was nearly universal prior to widespread glucocorticoid-use, but survival rates are now up to $90 \%$ at 5 years. Early steroid treatment is essential, so earliest possible diagnosis is needed. Rare diagnoses often present a diagnostic dilemma and recognition of this man's presenting constellation of symptoms as a syndrome was key to his diagnosis. While EGPA is classically characterized by the big three conditions--allergic rhinitis, asthma, and peripheral eosinophilia--multisystem organ involvement is common including peripheral neuropathy $(75 \%+)$, renal (80\%+) and dermatologic $(60 \%+)$ manifestations. Cardiac involvement is only present $15 \%$ of the time but is responsible for $50 \%$ of EGPA-attributable deaths. Recognition of EGPA as a possibility worth exploring in a patient with peripheral eosinophilia, asthma and multisystem disease can allow for more prompt diagnosis and treatment and minimize disease complications. 


\section{Delayed Symptoms of Miliary Tuberculosis in 90-year-old Japanese Woman with Fever after Hospitalization}

Y. Kanzawa, S. Mizuki, J. Ohnishi, K. Kawano, T. Nakajima, H. Seto, N. Ishimaru and S. Kinami

Department of General Internal Medicine, Akashi Medical Center, Hyogo Prefecture, Japan

Learning objectives: Miliary tuberculosis (TB) remains a diagnostic challenge. If a patient develops acute respiratory distress syndrome (ARDS) with unknown etiology, even in hospital, miliary TB should be considered. Dialogue between colleagues is important to establish true diagnosis.

Case information: A 90-year-old Japanese female became febrile without remarkable symptoms four days after surgery. She had been hospitalized with right humeral condylar fracture and underwent retrograde intramedullary nailing. She became febrile without remarkable symptoms on postoperative day (POD) 4. CT showed pulmonary edema and thickening of the gallbladder wall. Owing to suspicion of cholecystitis with heart failure or acute respiratory distress syndrome (ARDS), cefmetazole and furosemide were administered. She remained febrile, however, through POD 14. Aspiration pneumonitis was considered because of her advanced age, but CT on POD 14 was unremarkable. Respiratory condition gradually worsened and morphine was considered as palliative care. On POD 19, CT scan was once again performed as a final assessment, on the recommendation of a colleague. It revealed miliary shadows in the lungs (Figure) which had not been apparent previously. Sputum acid fast bacteria smear was positive and sputum and blood culture showed growth of Mycobacterium tuberculosis (TB). Final diagnosis was miliary TB. Although appropriate treatment was administered, the patient died.

Discussion: In our case, representativeness and premature closure were factors in diagnostic errors. We associated fever and respiratory failure in a 90-year-old female with aspiration pneumonia with ARDS and premature closure occurred. Miliary TB is often undetected in the early stages because progress is nonspecific and it does not readily suggest diagnosis. Miliary TB and ARDS are an uncommon combination but should be reportedly considered in any case of ARDS with unclear etiology, even if the patient becomes ARDS in hospital. Discussion with colleagues was key to eventual diagnosis.

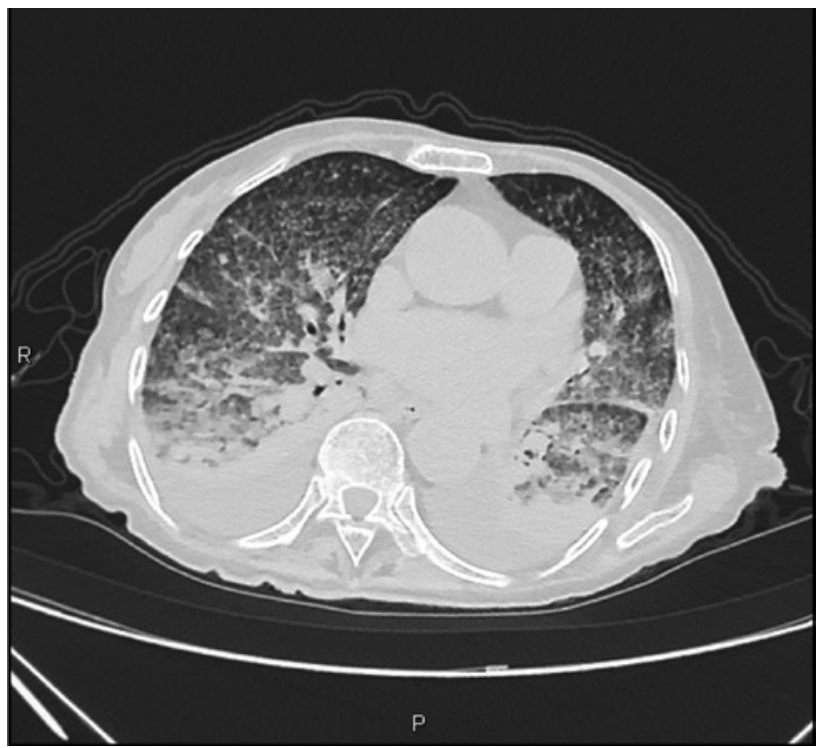

\section{Tissue is the Issue}

\section{Kitagawa}

Shonan Kamakura General Hospital

\section{Case information:}

Case1) A 62-year-old woman presented to our hospital with a progressive rash and high fever. She noticed a diffuse erythematous papules in her body and tingling sensations of her lower limbs two weeks prior to admission. Subsequently, she developed painful swelling of digital joints. On examination, erythematous papules and with various erosions of different crusts and size could be seen over the whole body except for her face. Also painful swelling in wrists, digital joints and knee were remarkable. The tingling sensation of finger tips and sole of her feet were shown. She did not exhibit mucosal involvement. The laboratory studies showed the following; white blood cell, 28400/ $\mu \mathrm{L}$ (neutrophil; $89.2 \%$ ); C-reactive protein, $29.1 \mathrm{mg} / \mathrm{dl}$. Immunological surveys of autoantibodies were negative, including antinuclear antibodies, antineutrophil cytoplasmic antibodies. Serum IgA levels, urinalysis and blood culture results were normal. 
Case2) A 42-year-old man presented to our hospital with a headache and high fever. He has no lymphoadenopathy and splenomegaly.The neurological examination were negative nuchal rigidity and negative Kernig's sign. The cell count of cerebrospinal fluid (CSF) at the time of admission is within normal limits. The laboratory studies showed the following; white blood cell, 10400/ $\mu \mathrm{L}$ (neutrophil; 70.2\%); C-reactive protein, $32.4 \mathrm{mg} / \mathrm{dl}$. His fever didn't improve after ten days in hospital.Immunological surveys of autoantibodies were negative, including antinuclear antibodies, antineutrophil cytoplasmic antibodies. Serum IgA levels, urinalysis and blood culture results were normal. For better assessment of their symptoms, we performed skin biopsy, which showed leukocytoclastic vasculitis. Based on her pathological and laboratory findings, without specific histories such as medications, diagnosis of unclassifiable leukocytoclastic vasculitis had been made.

Discussion: Leukocytoclastic vasculitis (LCV) is a rare type of small-vessel vasculitis with a reported incidence rate of 30/1,000,000. The diagnosis of LCV is often challenging due to its various modes of presentation as this case showed, however, skin biopsy is a useful diagnostic tool in this case. Encountering cases that show a variety of symptoms with skin involvement, we should try to perform a skin biopsy.

\title{
Frozen and Poisoned: Salicylate Toxicity in the Altered Hypothermic Patient
}

Ł. Koes and J. Lu

John H. Stroger Hospital, Cook County Health and Hospitals System, Department of Emergency Medicine

\section{Learning Objectives:}

- $\quad$ Describe three physiological mechanisms of acetylsalicylic acid

- Analyze an atypical presentation of salicylism

- $\quad$ Appraise the utility of toxicological screening in undifferentiated altered patients

Case: A 75-year old female was found in the street by EMS, altered and combative, in minus 4ㅇF weather. Blood glucose was $87 \mathrm{mg} / \mathrm{d}$. She presented grunting, opening eyes to noxious stimuli, and withdrawing from pain - GCS 8 (E2V2M4). VS: 85.2ㅇF (rectal), HR 110, BP 96/61, RR 10, SaO2 96\%. Exam showed small pupils and non-labored respirations with clear lung sounds. She was intubated within 30 minutes for airway protection. Head CT was negative. Notable initial labs: WBC $19.4 \mathrm{k} / \mathrm{uL}$, sodium $146 \mathrm{mEq} / \mathrm{L}$, chloride $100 \mathrm{mEq} / \mathrm{L}$, bicarbonate $23 \mathrm{mEq} / \mathrm{L}$, creatinine $1.1 \mathrm{mg} / \mathrm{dL}$, and an anion gap (23 mEq/L). After intubation, toxicological screening unexpectedly showed an elevated salicylate level (80.4 mg/dL). She was transferred to the ICU for hemodialysis but succumbed to cardiopulmonary arrest four hours after presentation despite resuscitative efforts.

Discussion: Salicylate toxicity is classically recognized by the triad of hyperventilation, ototoxicity, and gastrointestinal distress. The primary mechanisms of action of acetylsalicylic acid include direct stimulation of medullary respiratory centers, uncoupling of oxidative phosphorylation leading to hyperpyrexia, and metabolic acidosis secondary to anaerobic metabolism and substrate accumulation. Early in acute salicylism, hyperventilation causes respiratory alkalosis, which buffers the developing metabolic acidosis. Ultimately, as the compensatory mechanisms of the body fail or are removed, profound acidosis leads to pulmonary/cerebral edema, myocardial/CNS depression, and arrest. Toxicological etiologies other than ethanol are implicated in up to $6.1 \%$ of undifferentiated altered patients. In this patient, salicylism was masked by profound hypothermia and altered mentation. Routine toxicology screening is often non-contributory and its interpretation requires nuanced understanding about the tested substances. In this situation, toxicology testing was pivotal in revealing a diagnosis that directly affected management and outcome.

\section{When We Listen To Each Other, Everyone Wins: A Nurse’s Experience Saving a Patient from an Unnecessary Procedure}

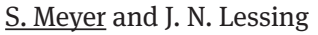

\section{Learning Objectives:}

- $\quad$ Effective communication between all team members is essential to avoiding errors and to providing excellent patient care.

- $\quad$ Nurses serve as front-line members of the care team. Nurses, and all team members, must be and feel empowered to speak-up on behalf of our patients, and physicians are wise to listen to their nurses' concerns.

Case Information: Being a nurse on the Acute Care for the Elderly service is challenging, which makes effective communication and teamwork essential for ensuring best care for our vulnerable patient population. I was the nurse caring for an 85 year-old woman admitted overnight for reported symptomatic hematochezia. Due to advanced dementia, she was unable to contribute to providing her history. Based on symptoms and significant hemoglobin drop, the physician requested bowel prep for next-day colonoscopy. While toileting the patient, I realized the bleeding source was from her vagina, not rectum. I alerted the physician and upon further inspection, we verified the patient did not have a gastrointestinal bleed, but rather uterine bleeding.

Discussion: Because of team communication and prompt response, this patient was spared an unnecessary procedure. Providing quality patient care requires teamwork, which is based on effective communication and mutual respect. Constructive collaboration leads to improved clinical performance and patient outcomes, fewer medical errors, and increased patient satisfaction. 1,2 Communication failures are significant contributors to medical errors. Among sentinel events reported to the Joint Commission from 1995-2004, communication failures were the leading root cause, greatest contributor to treatment delays and medication error, and a leading cause of fatal falls.1 
When nurses and all team members ARE and FEEL empowered to speak-up on behalf of our patients, patient care improves. Team collaboration and communication should be seen as opportunities to reduce errors.

\title{
References:
}

1. O’Daniel M, Rosenstein AH. Professional Communication and Team Collaboration. In: Hughes RG, editor. Patient Safety and Quality: An Evidence-Based Handbook for Nurses. Rockville (MD): Agency for Healthcare Research and Quality (US); 2008 Apr. Chapter 33. Available from:https://www.ncbi.nlm.nih.gov/books/NBK2637/

2. Institute of Medicine (US) Committee on the Work Environment for Nurses and Patient Safety; Page A, editor. Keeping Patients Safe: Transforming the Work Environment of Nurses. Washington (DC): National Academies Press (US); 2004. Appendix B, Interdisciplinary Collaboration, Team Functioning, and Patient Safety. Available from:https://www.ncbi.nlm.nih.gov/books/NBK216180/

\section{Delayed Diagnosis of Linezolid-Induced Lactic Acidosis by Search Satisficing and Limited Knowledge}

\author{
S. Mizuki, Y. Kanzawa, K. Kawano, T. Nakajima, H. Seto, N. Ishimaru and S. Kinami \\ Department of General Internal Medicine, Akashi Medical Center, Hyogo, Japan
}

Learning objectives: Linezolid-induced lactic acidosis (LILA) is rare, but can lead to critical adverse events. Whether patient's new symptoms are the result of our prescription should always be considered so not to miss a rare disease caused by adverse drug reaction.

Case information: A 73-year-old male had orthopedic surgery for lumbar hernia. Medical history included diabetes mellitus, but no liver injury or renal disease. Medication did not include metformin. After surgery, he had surgical wound infection including pyogenic spondylitis, epidural abscess and continuous bacteremia caused by methicillin-resistant staphylococcus aureus. Vancomycin and daptomycin in addition to drainage could not control his illness. The causative microorganism became resistant to vancomycin and daptomycin, so we started linezolid as an alternative. Blood culture became negative for causative microorganisms on the day of initiation of linezolid. Three weeks after initiation of linezolid, the patient had nausea and gradual reduction of his dietary intake. The cause of his symptoms could not be identified in blood tests or imaging studies. As the patient looked to be in healthy condition, we made diagnosis of diabetic gastroparesis. Five weeks after initiation of linezolid, however, he suddenly lost consciousness and his blood pressure dropped rapidly. Blood gas test revealed extreme metabolic lactic acidosis. We made new diagnosis of LILA. After cessation of linezolid and continuous hemodiafiltration, the patient recovered. Discussion: Despite a mortality rate of over 20\%, LILA is rare and data on its adverse effect are very limited. Abdominal symptoms are common. Our case had typical clinical presentation, but owing to search satisficing and limited knowledge of LILA, we did not reach correct diagnosis. To avoid these mechanisms, when a patient presents new symptoms, whether they come from adverse drug events should be considered, and differential diagnoses should be exhaustively listed.

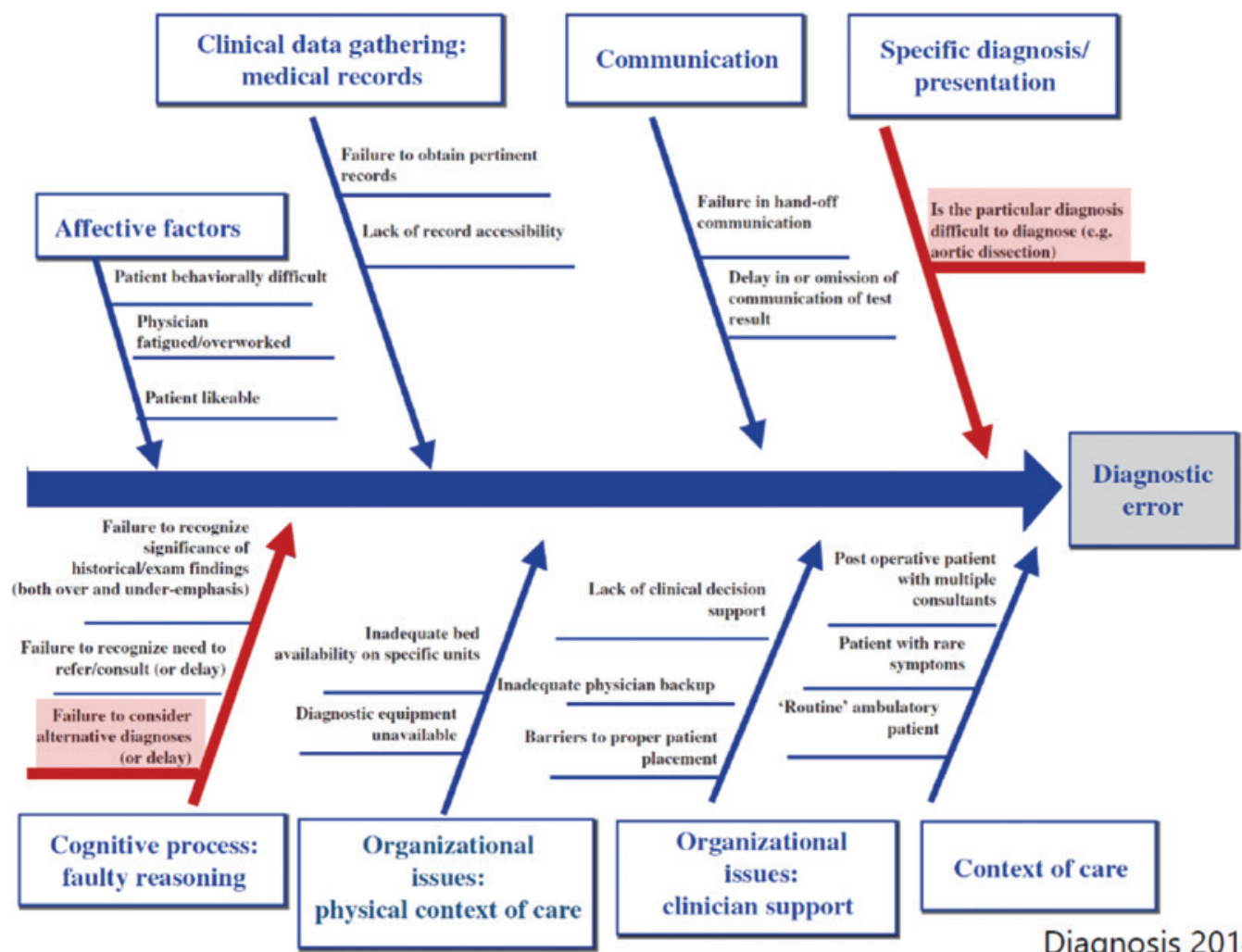




\title{
The Expediency of Reassessment of Spinal Magnetic Resonance Imaging for Vertebral Osteomyelitis
}

$\underline{\text { H. Osamu }}^{1}$, S. Nishiguchi ${ }^{2}$, J. Branch ${ }^{1}$ and I. Kitagawa ${ }^{1}$

${ }^{1}$ Shonan Kamakura General Hospital

${ }^{2}$ Hayama Heart Center

Learning objectives: Continuing to suspect vertebral osteomyelitis if the initial spinal magnetic resonance imaging scan is negative, and reassessing it after two to four weeks.

Case information: A 66-year-old Caucasian gentleman had a preceding history of fever up to $38^{\circ} \mathrm{C}$ and pharyngitis. Rigors occurred followed by severe low back pain after several days. An increase of fluid intake resulted in polyuria and nocturia. Initial physical examination revealed only prostate tenderness. He demonstrated a leukocytosis of $12,200 / \mu \mathrm{L}$ and the C-reactive protein was $8.86 \mathrm{mg} / \mathrm{dL}$. Urinalysis was unrevealing. An abdominal computed tomography scan showed prostatomegaly. A spinal magnetic resonance imaging (MRI) scan was normal. He was diagnosed with acute prostatitis and prescribed intravenous ceftriaxone. Despite symptomatic improvement, on the third day of hospitalization his pre-admission blood cultures grew streptococcal spp. This was though unlikely as the case of acute prostatitis. Because this history was consistent with disseminated bacterial infection from a likely pharyngitis, a spinal MRI scan was repeated which showed a new high intensity area at the L1 to L2 vertebral body and intervertebral disc. He was finally diagnosed with vertebral osteomyelitis (VO). A transesophageal echocardiogram was performed for infective endocarditis, but no vegetation was evident. He received six weeks of intravenous treatment followed by oral antibiotics.

Discussion: Generally, in cases of VO, the spinal MRI scan shows typical findings. If there are no abnormalities present, it is possible to misdiagnose early disease. The bacteria may spread by the hematogenous route from a focus of infection such as pharyngitis and infective endocarditis. In cases where an initial MRI does not show typical findings for VO, a repeat MRI scan should be repeated within two to four weeks after the onset of symptoms.

\section{You Can’t Judge a Book by its Cover!}

\section{Osawa, S. Tobe and K. Nakano}

Shonan Fujisawa Tokushukai Hospital

Learning Objectives: Bilateral pleural effusion is not an uncommon finding in clinical practice. But bilateral pleural effusion with different origin on each side is rare.

Case information: The case is a 74-year-old man with a chief complaint of dyspnea. Two hours ago, pale face, dyspnea and leg edema had begun. On arrival to the ER, he had hypoxemia and chest x-ray showed bilateral pleural effusion. Because the patient had cardiomegaly and leg edema, at first diagnosis of chronic heart failure was made. At ER, thoracentesis was performed on the right side and the result showed exudate effusion. After admission, because the effusion was limiting oxygenation, thoracentesis on the left side was performed. Left pleura effusion was red and suggested hemorrhagic effusion. Left rib fractures were seen on the chest CT scan performed on admission. History taking revealed that the patient had dementia and often episodes of trip and fall.

Discussion: Bilateral pleural effusions nearly always have the same cause, however in rare cases there may be two separate causes. This is known as Contarini's syndrome (1). On this case, right side effusion resulted from chronic aspiration pneumonia and left side effusion resulted from chest trauma. Though no apparent harm was done to the patient, careful history taking and physical examination added with accurate image interpretation may have prevented delay in diagnosis. We must never take things for granted.

\section{Sickle Cell, the Great Imitator}

\author{
$\underline{\text { S. Raj }}{ }^{1}$, K. Leyens ${ }^{2}$ and A. Dakroub ${ }^{2}$ \\ ${ }^{1}$ University of Pittsburgh School of Medicine \\ ${ }^{2} \mathrm{UPMC}$
}

\section{Learning Objectives:}

- $\quad$ Recognize how sickle cell masked the underlying pathology

- Identify the cognitive biases that led to delayed diagnosis

Case: 23-year-old female with a history of HbSS Sickle Cell Disease, CVA, Gadolinium contrast allergy, ovarian cysts, and monthly hospitalizations for vaso-occlusive crisis (VOC) who presented with back pain, chest pain, and shoulder and knee pain.

Patient noted that these symptoms were similar to prior presentations. She had no pulmonary, neurologic, or cardiovascular symptoms to suggest other etiologies. Upon chart review, it was found that she was admitted two weeks prior for VOC with resolution of all pain except abdominal pain. When asked, the patient stated that it disappeared with her menstrual cycle. Prior non-contrasted (due to allergy and 
steroid-induced pain) CT scans, MRIs, and pelvic ultrasounds only showed a large ovarian cyst. Repeat pelvic ultrasound showed hypoechoic lesions within the ovaries suggestive of either hemorrhagic cysts or endometriomas. Following steroid and diphenhydramine pretreatment, contrast pelvic MRI showed stage 4 endometriosis.

Discussion: This case illustrates how one pathology can be masked by another and how cognitive biases can delay diagnosis and treatment. Of note, she did not present with symptoms of endometriosis at the time of presentation; rather, inquiry into prior presentations helped lead to a diagnosis.

Several cognitive biases played a role in prematurely diagnosing this as VOC and delaying the appropriate diagnosis, specifically anchoring bias, framing bias, and availability bias. Anchoring bias was present when the patient stated, "I am having a sickle cell crisis." Framing bias was present with her history of prior hospitalizations and complex care plan defining treatment, and when the ED signed out the patient. Availability bias was present when the EMR was opened with prior hospitalizations showing VOC.

Her contrast allergy also hindered diagnosis. Prior studies had insufficient sensitivity, and new scans were not obtained due to the presence of prior scans and worsening VOC with steroid pretreatment.

\section{A Puzzling Painful Eye}

$\underline{\text { S. Sandroni }}$

Florida State University College of Medicine

Learning Objective: Consider the intersection of cognitive biases and incomplete patient information

Case: A 67 year old woman presented to an ophthalmologist complaining of severe pain in her left eye of 24 hours duration. She denied trauma.

Past history: She took medication for hypertension and hypothyroidism, and weekly allergy injections for pollens and animal dander. She had undergone bilateral cataract surgery one year earlier.

Exam: diminished visual acuity left eye, inflamed conjunctiva, Otherwise normal. No evidence of infection, foreign body, corneal abrasion, corneal tear, or glaucoma.

Diagnostic process: The ophthalmologist said that prior to seeing the patient he was expecting some injury related to fireworks given that the pain had started on July 4. However, when pressed by his nurse to select a diagnosis he said it might be an allergic reaction, having eliminated other more common causes. He said he would prescribe some steroid eye drops. At this point the patient volunteered that she had had an allergic reaction to a particular brand of steroid eye drops after the cataract surgery. The ophthalmologist then asked if the patient had experienced any recent steroid exposure.

She recalled having been bitten by some ants while gardening a few days earlier, which resulted in painful pruritic papules on her fingers. She self medicated these with a hydrocortisone ointment several times, and said she may have rubbed her eyelids as she was also experiencing other allergic symptoms at the same time. A different formulation of steroid drops was prescribed, and her symptoms resolved over the next 48 hours. Discussion: This unusual case of severe eye pain demonstrates the challenges presented by two types of cognitive bias, as well as the benefit of additional history provided by the patient. The July 4th timing of the event caused the physician to anchor initially on the probability of a fireworks-related injury, and the availability heuristic led to focus on other more commonly seen diagnoses. When these diagnoses were excluded the physician tentatively considered a less common diagnosis, and this led the patient to volunteer crucial additional information which she had not originally linked to her problem. The communication of the physician's uncertainty to the patient facilitated a dialog which led to the correct diagnosis.

\section{Reference:}

Saposnik G, Redelmeier D, Ruff C, Tobler P: Cognitive biases associated with medical decisions: a systematic review. BMC Med Inform Decis Mak 2016; 16: 1381

\section{Liver Abscess Due to Pneumonia may Metastasize to Other Organs}

M. Sato, S. Masaji, T. Wakai, S. Constantine and N. Urara

Sapporo Tokushu-kai Hospital, Sapporo, Japan

The University of Michigan, Department of Family Medicine, Ann Arbor, MI

Learning objective: Liver abscess may metastasize to other organs, especially due to K. Pneumonia infection, and workup to find other abscess until proven otherwise is essential.

Case information: Patient is 80-year-old male with history of Parkinsonism presented with fever. He went to a clinic for fever 3 days prior to hospitalization, and supportive therapy was recommended. The fever persisted with hypotension at the clinic and he was transferred to Sapporo Tokushu-kai hospital. On admission, he was found to have hypotension with low grade fever. Bilateral infiltrate in chest CT was found which implied pneumonia. Ampicillin/Sulbactam was initiated. Abdominal CT revealed possible abscess (17X14mm). Blood culture 
came back positive for K. Pneumoniae 3 days after hospitalization. Repeated abdominal CT was performed 11 days after the hospitalization, which revealed smaller abscess, and 4 weeks of intravenous antibiotics was planned. However, patient started to have high fever 28 days after hospitalization. The antibiotics was switched to broader spectrum. The blood culture came back positive for K. Pneumoniae again. Low grade fever sustained and CT of abdomen revealed psoriatic abscess and subdural abscess 45 days after hospitalization. Drainage of those abscess was performed, followed by 6 weeks of the intravenous antibiotics. Pt was discharged to rehabilitation facility 115 days after the hospitalization.

Discussion: Pyogenic liver abscess was suspected in initial CT. However, drainage was not performed because of the size. The abscess with smaller size $(<3 \mathrm{~cm})$ may be treated with antibiotics alone, and the antibiotics was effective. However, when the fever flared, broader spectrum antibiotics was initiated but repeated CT was not performed to search for other possible abscess. From 8 to $30 \%$ of K. pneumonia induced liver abscess metastasize to other organs. Even with the improvement of the liver abscess, the metastasis to the other organs were initially missed due to premature closure.

\title{
One Foot Forward, Two Steps Back
}

\author{
L. C. Schwartz, S. Desai-Oghra and M. Moussa \\ NYU Langone Hospital - Brooklyn
}

Learning Objectives: Radiologist interpretations contribute to anchoring bias.

Differentiating problems with similar presentations requires thorough exams and detailed patient histories. Correct management often requires a patient-centered approach.

Case Information: A 48 year old woman developed a limp from pain in the ball of the right foot while training for her fifth half marathon. A radiologist diagnosed Freiberg's Disease Stage 2, a rare avascular necrosis of the metatarsal head, based on an x-ray ordered by her podiatrist. X-ray findings include flattening of the metatarsal head, which is a normal variant in $10 \%$ of people. The podiatrist prescribed a fracture boot. The patient consulted an orthopedist who changed management to a metatarsal pad for her insole after reviewing the x-ray without an alternative diagnosis. Doubting her diagnosis, the patient consulted a physiatrist who confirmed Freiberg's Disease.

After 6 weeks of pain, her orthopedist ordered an MRI that ruled out Freiberg's and showed a partial plantar plate tear with significant localized bursitis. She was taught to tape her toe, but pain persisted with the metatarsal pad. A new podiatrist noticed that the metatarsal pad was creating gait problems and he altered her running shoe insole instead. After two weeks, she was running again.

Discussion: Metatarsalgia has many causes, yet three doctors anchored their diagnosis on an incorrect radiology report. Listening to the nuances of the patient's story and performing an extensive exam may have expedited the correct diagnosis. Many doctors use metatarsal pads, but this management may cause harm in some patients.
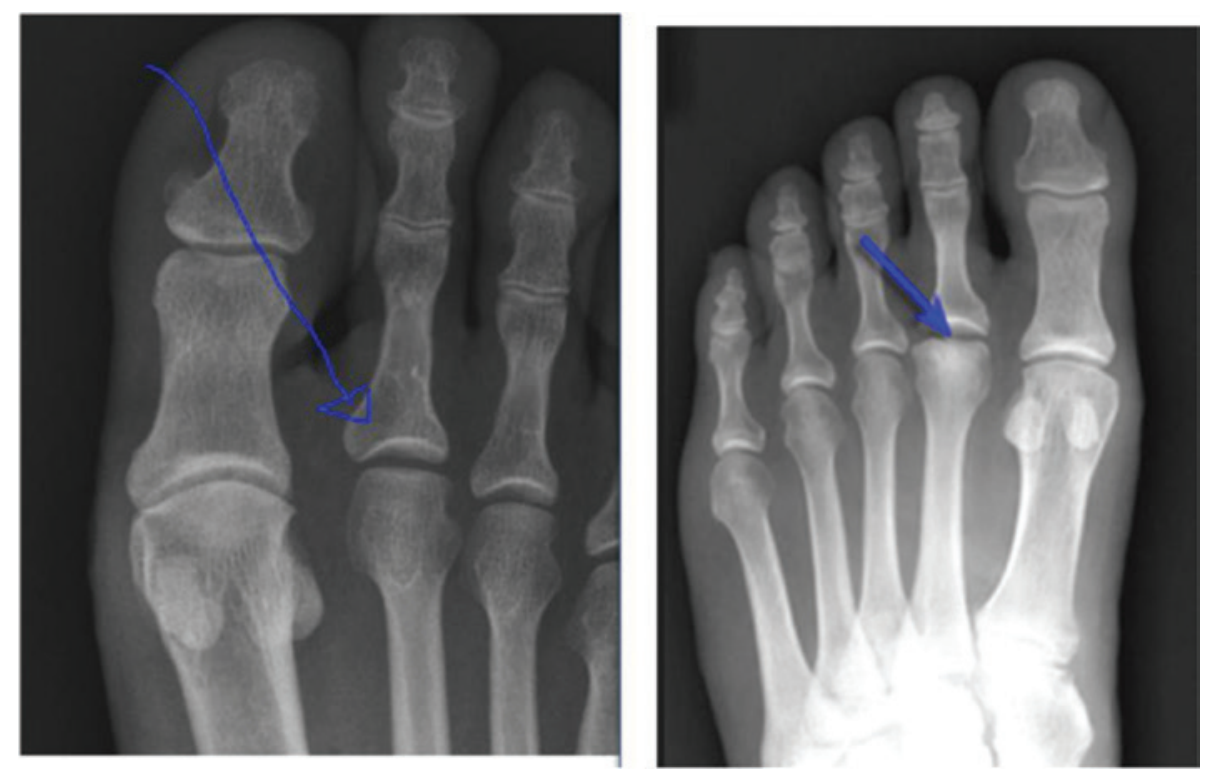


\section{'Hand-over' Times are Dangerous Times in the Emergency Department and the 'Halo Effect' Lingers Here}

E. Seow and F. Lee

Khoo Teck Puat Hospital, Singapore

Case: 73 year old lady presented to the Emergency Department complaining of:

- $\quad$ Pain over her right thigh which radiated to her lower back associated with fever and chills; sudden onset and occurred the night before

- $\quad$ Patient had tripped and fallen forward 2 weeks prior to this presentation.

Past medical history:

- $\quad$ Right total knee replacement 9 months prior to presentation.

- $\quad$ hypertensive on medication.

On examination: These were her vital signs:

- $\quad$ Temperature 37 degrees Celsius, heart rate 115 per minute, blood pressure 110/71, oxygen saturation on room air was 99\%.

- Patient was alert but in pain.

- Her right thigh was indurated and erythematous.. This area was not warm or tender. Her right labia majora was swollen.

The provisional diagnoses by the junior doctor who first attended to this patient were:

- $\quad$ Right thigh hematoma

- Fracture

- Necrotizing fasciitis

An X-ray of the right thigh and pelvis excluded a fracture. Before the X-ray was shot, the patient had to remove medicated plasters from the right thigh after which it was noted that there was a skin tear with blister formation and surrounding erythema in the same area subsequently. The patient was reviewed by a senior doctor who noted that the calculated LRINEC (laboratory risk indicator for necrotizing fasciitis) score was 6 (CRP (4) and creatinine (2)).

A second senior doctor reviewed the patient before she was admitted. A diagnosis of deep vein thrombosis was considered. A POCUS was conducted but was inconclusive

The patient was admitted to the medical unit and was referred to the orthopaedic team.

The patient passed away 7 hours after admission.

The cause of death: Necrotizing Fasciitis.

\section{Learning points:}

1. A patient especially an ill-patient that is handed over from one team of doctors to another should have its problems "scrubbed". The differential diagnosis and the conclusion reached by the previous team and the reasoning supporting it should be reviewed. The "halo effect' can interfere with this process.

2. The doctors did not recognize or were not aware of the early physical signs that were present strongly pointing to the high likelihood of necrotizing fasciitis i.e. skin tear and blister formation, erythema after the removal of a medicated plaster - clinical acumen is still important.

3. Failure to recognize that the LRINEC score is a rule-in score and not a rule-out score. This patient should have been referred to the Orthopedist while in the emergency department.

\section{Bad Boyz Will Be Bad? Social Biases May Manifest as Cognitive Biases}

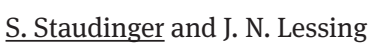

University of Colorado

Learning Objectives: 1) Recognize dialysis adequacy should not be measured by resolution of uremia nor reduction in BUN alone. 2) Be aware that biases can affect a clinician's thought process and decision-making.

Case: A 45-year-old African-American homeless man with a history of ESRD, HTN, cocaine abuse, opiate seeking and abusive behavior toward staff was admitted for the fifth time that year for hyperkalemia and hypertensive urgency in the setting of suspected dialysis and medication noncompliance. Despite inpatient hemodialysis, refractory hypertension (attributed to cocaine use) and hyperkalemia (attributed to dietary noncompliance) persisted. Eventually, post-HD hyperkalemia led the team to consider that dialysis was inadequate. A fistulogram revealed collaterals and subclavian stenosis, correction of which led to effective dialysis and resolution of hypertension and hyperkalemia. Despite this, sadly, he died from a fatal arrhythmia 2 months later. 
Discussion: The patient's drug use and noncompliance likely introduced implicit bias that caused multiple providers to misattribute evidence of ineffective dialysis. Assessment of dialysis adequacy by symptom resolution or improvement in BUN is inadequate, as the dialysis dose which reduces uremic symptoms is lower than that which improves survival (PMID 8366899). This patient might commonly be labeled a "difficult patient” or a "frequent flyer", labels which convey visceral bias and impose risk of premature closure. Studies have shown that providers' implicit biases with respect to race, substance use or other characteristics may contribute to health disparities by affecting provider attitudes and subsequent care (PMIDs 23576243, 23490450). Interactions with "difficult” or noncompliant patients and substance users often evoke provider frustration or defensiveness which patients may perceive as intentional mistreatment (PMID 12047728). Furthermore, patients who feel stigmatized may delay or avoid medical care, perpetuating "noncompliance". Awareness of the impact of these implicit biases and interest in providing equitable care for all regardless of circumstance is necessary to provide the type of medical care we strive to provide and that all patients deserve.

\section{A Case of Pancreatic Tail Cancer Presenting with Bilateral Chronic Scrotal Pain}

\section{Suzuki}

Sendai Medical Center

Learning objectives: Chronic scrotal pain is defined as continuous or intermittent pain in the testes for 3 or more months. For chronic scrotal pain, spermatic cord metastasis of pancreatic cancer should be considered as a differential diagnosis.

Case information: A 75-year-old man visited our hospital complaining of scrotal pain persistent for 3 months. He had chronic low back pain and benign prostatic hyperplasia. Family history showed that his sister due to pancreatic cancer. He complained of continuous dull pain, which gradually exacerbated in both scrotums 3 months before his visit. Physical examination showed no abnormality, including testicular swelling and straight leg raise test on both sides. Further, there were no abnormalities in the blood test results. Magnetic resonance imaging of the lumbar spine showed no abnormal findings. He returned home without a compatible diagnosis but re-visited when his symptoms continued. An abdominal contrast CT showed a 3-cm mass of pancreatic tail with lymph node swelling and many nodules suspected of peritoneal dissemination. Based on these findings, we diagnosed peritoneal dissemination of pancreatic tail cancer and spermatic cord metastasis.

Discussion: The causes of this diagnostic error were 1) availability bias (chronic low back pain) and 2) confirmation bias (ignored family history of pancreatic cancer). We reviewed 14 Japanese cases of spermatic cord metastasis of pancreatic cancer; of which 11 cases were caused by pancreatic tail cancer. Nine out of these 14 cases metastasized to the right, three to the left, and two to both sides of the spermatic cord. The transfer pathway of pancreatic cancer to the spermatic cord remains unclear. Pancreatic cancer is a poor symptomatic disease, which makes its early diagnosis difficult. For chronic bilateral scrotal pain, spermatic cord metastasis of pancreatic cancer should be considered.

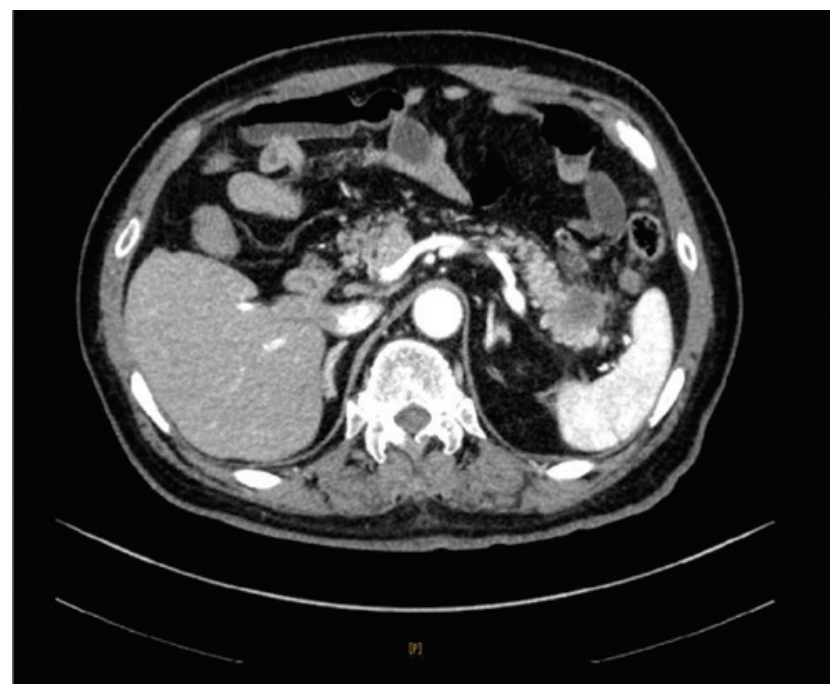




\title{
A Frame of Mind: Misdiagnosis of Stroke Symptoms Resulting in Unnecessary Thrombolytic Administration
}

\author{
D. Varela, L. Officer and J. Lessing
}

University of Colorado School of Medicine Department of Internal Medicine

\section{Learning objectives:}

- Symptoms stemming from unrelated medical conditions can lead to misdiagnosis of a single condition.

- Framing matters- Prioritized differential diagnosis for common chief complaints can drastically depend on setting.

- Setting matters- history and physical performed in a hectic and time-sensitive setting, such as in evaluation for stroke, can increase risk of making a diagnostic error.

Case Information: A 65-year old man with hyperlipidemia and coronary artery disease presented to the emergency department (ED) with acute onset headache, neck pain, and vertigo after changing positions during yoga. Nystagmus and truncal ataxia led to head/neck CT angiogram, which showed no evidence of dissection, bleed, or vertebrobasilar insufficiency. Thrombolytic therapy was administered for concern of posterior circulation stroke.

Brain/spine MRI later showed only C4-C7 spinal stenosis. Additional history revealed the vertigo occurred only when rising or rolling. Additional examination revealed nystagmus during Dix-Hallpike maneuver, leading to diagnosis of benign paroxysmal positional vertigo (BPPV), with concurrent neck pain from cervical stenosis. Vertigo improved with canalith repositioning. The reported truncal ataxia was not observed after admission from the ED, though he was unsteady on his feet during episodes of vertigo. It was felt that the "truncal ataxia" may have actually been disequilibrium secondary to the vertigo. He was discharged after his symptoms resolved and after he failed to show signs of any bleeding complications.

Discussion: A combination of symptoms (headache, neck pain, vertigo, and ataxia), interpreted individually, may not raise as much alarm for stroke as when considered collectively. Initial evaluation in the ED, where stroke is seen more commonly than in the ambulatory setting, likely affected perceived pre-test probability of this do-not-miss diagnosis. Priority on timely diagnosis of stroke to stay within the thrombolytic window not unreasonably led to reduced time for history-taking, diagnostic maneuvers, and deeper consideration of less grave conditions. Misdiagnosis with posterior circulation stroke led to thrombolytics for what was ultimately found to be BPPV and cervical stenosis.

We are taught the rule of parsimony-the simplest explanation is usually right-but this is not always correct. Sometimes more than one diagnosis explains the constellation of unrelated findings. Recognition of framing effect (Croskerry 2003) can help reduce error and improve diagnostic accuracy.

\section{The Importance of Direct Communication in Diagnosis: A Case of Non-Improving Sinus Symptoms}

\author{
T. (McLernon) Ward and J. N. Lessing
}

University of Colorado, Denver, CO

Case information: A 48 year-old man with history of facial trauma and remote intranasal drug-use presented to our academic center for further evaluation of necrotic nasal passage destruction and oronasal fistula despite multiple sinus surgeries, now with cultures positive for MRSA and Candida. Previous biopsies and blood markers were reported negative for midline lethal granuloma. New nasal endoscopic biopsies showed inflammation, necrosis and occluded blood vessels. Invasive fungal infection, autoimmune vasculitis, leishmaniasis, and IgG4-process were ruled-out. Without another better explanation, inhalation drug-use and possible superimposed infection was thought to be the etiology of this unusually destructive lesion. However, in the absence of expected improvement and persistent concern for lymphoma, additional stains were specifically requested and based on the results the pathology report was changed from benign to malignant extranodal NK/T-cell lymphoma. Discussion: Natural killer (NK)/T-cell lymphoma is a type of non-Hodgkin lymphoma. While rare, sinus symptoms are common. This case highlights how diagnostic momentum and premature closure based on negative studies can result in delayed diagnosis. Previous biopsies reportedly negative for malignancy led us away from a diagnosis of lymphoma. However, we at first did not recognize that NK/T-cell lymphoma requires special stains that must be specifically considered or requested. Moreover, the previous reported biopsies did not have these stains performed both due to unintentional and intentional omission, as some biopsies were necrotic. Recognition that pathology tests (like all tests) are influenced by a pathologist's knowledge and understanding of the clinical case was key to reaching the correct diagnosis. It was only after pathologists and clinicians directly discussed the case, that the appropriate stains were performed and the correct diagnosis was reached. 


\title{
Granulomatosis with Polyangitis: Navigating a Rare Diagnosis in a Fragmented System
}

\author{
C. Wasserman and K. Joshi \\ Baystate Medical Center
}

Learning Objectives: Prompt diagnosis of Granulomatosis with Polyangitis (GPA) is often a challenge due to the presentation heterogeneity, variety of organs affected and disease rarity. This case highlights the ways in which fragmented care can further interfere with early diagnosis, and emphasizes the need for cohesive communication between physicians.

Case: This is a 59 year old female with history of Asthma, Chronic Cough and Nephrolithiasis presenting with acute respiratory failure status post intubation with desatturations despite vent setting optimization requiring subsequent transition to VA ECMO. In June 2018, the patient was diagnosed with ureterolithiasis and during hospitalization was incidentally found to have pulmonary nodules on CT. Documentation reflects follow up pulmonology visit addressed asthma, exertional dyspnea and chronic cough without mention of recent imaging. In January 2019, the patient presented to PCP for an annual appointment and was found to have isolated hematuria, attributed to probable nephrolithiasis. In February, the patient was seen by PCP for microcytic anemia and was referred to pulmonology to address previous pulmonary nodule findings. Unfortunately, she did not make it to the pulmonology appointment prior to her unfortunate presentation in April. While on ECMO, PR3 and c-ANCA levels returned positive and patient was confirmed to have GPA.

Discussion: GPA is a disease involving systemic necrosis and inflammation of vessels, most commonly affecting the upper and lower respiratory tract, as well as the kidneys. Despite its rare presentation, the consequences of delayed diagnosis and treatment can be life threatening. Communication between physicians was non-existent despite being in the same EMR network and, as a consequence, the opportunity to make an early diagnosis was missed. The unfortunate outcome makes a case for the utility of generating electronic conversations between physicians within the same EMR network.

\section{Thinking Outside the Cardiac Box: Anchoring in an Elderly Patient with Multiple Visits for Orthopnea}

\author{
T. K. Zangara, A. Hudler, T. Anstett and W. Silkworth
}

University of Colorado School of Medicine at Anschutz Medical Campus

\section{Learning Objectives:}

- Recognize that neuromuscular disorders can mimic respiratory illnesses

- Include a neurological assessment in the comprehensive workup for dyspnea of unknown etiology

Case Information: A 77-year-old male presented to the emergency department (ED) four times over a four-month period for evaluation of fatigue and progressive shortness of breath characterized as orthopneic. His past medical history included hypertension and recently diagnosed prostate cancer for which he was taking antiandrogen therapy. During his first visit to the ED, he was discharged with a diagnosis of dyspnea of unknown etiology. At his following visit, he was admitted and underwent a cardiac workup consisting of a nuclear stress test and left and right cardiac catheterizations, which were unrevealing. During his third visit, he was discharged from the ED with a diagnosis of pneumonia but returned six hours later for worsening dyspnea. Oxygen saturation by pulse-oximetry revealed mild hypoxia of $90 \%$ on room air while upright and 79\% while supine with associated severe dyspnea. The patient was admitted and workup was negative for pneumonia, chronic obstructive pulmonary disease, heart failure, pulmonary shunting, and dysphagia. Detailed examination revealed fasciculations and weakness of the upper extremities, and hyperreflexia in the upper and lower extremities. Further discussion revealed four months of progressive weakness and several years of worsening tremors. Pulmonary function testing demonstrated reduced inspiratory strength and electromyography confirmed the diagnosis of amyotrophic lateral sclerosis (ALS).

Discussion: Dyspnea and fatigue are frequent complaints in outpatient settings. Though often due to cardiac dysfunction, the differential diagnosis for orthopnea is limited but includes neuromuscular disease. This case illustrates the importance of maintaining a broad differential and considering a neurologic etiology for dyspnea[1]. Although ALS currently has no cure, misdiagnosis can lead to delayed treatment, over-testing, reduced quality of life, and emotional distress for patients and their families.

\section{References}

1.) Pavletic AJ, Hnatiuk O. Puzzling dyspnea caused by respiratory muscle weakness. J Am Board Fam Med. 2012;25:396-7. doi: 10.3122/ jabfm.2012.03.110220. 


\section{Education}

\section{Attitudes of Residents in the Diagnostic Process: A Survey of Spanish-Speaking Residents}

L. A. Carrión ${ }^{1}$, M. C. Jurado ${ }^{2}$, A. S. Sempere 3 , L. O. Fernández ${ }^{4}$ and M. I. Campos ${ }^{4}$

${ }^{1}$ Asociación Foro Osler

${ }^{2}$ Centro de Salud Federica Montseny. Dirección Asistencial Sureste de Madrid.

${ }^{3}$ Centro de Salud Ghandi. Dirección Asistencial Este de Madrid.

${ }^{4}$ Servicio de Oncología Médica Hospital Clínico Málaga.Servicio Andaluz de Salud

Purpose/Problem: To know the opinión of a sample of residents about the diagnostic process and clinical reasoning.

Description of the study: We elaborate an electronic questionnaire with "Google Forms", with questions about their clinical practice and clinical reasoning habits, every question with several responses, but just one allowed. We sent the questionnaire to medical residents from two major hospitals in Málaga, Spain, but also we open the poll in internet through Facebook and twitter to Spanish and Portuguese speaking residents.

Outcome: We collected a total of 190 valid responses, 70\% from Europe and 30\% from South and Central America, with 85\% coming from medical specialties. Below, we present some preliminary results.

- When we asked how they acted in the anamnesis, 53\% answered that they worked with probability and 28\% looked for key symptoms. Only $7 \%$ admitted that they did not elaborate a new clinical history.

- When we asked which aspect they considered the most important to identify a clinical problem, semiology with the $55 \%$ of responses was the preferred option, followed by an attempt to organize information in syndromes and in $9 \%$ working as a team.

- When we asked about the Differential Diagnosis, 86\% thought that is a procedure that increases patient safety, but $8 \%$ will wait first for the results of the tests.

- When we asked about how they elaborate a Differential Diagnosis, $40 \%$ compares the data with their mental schemas, $24 \%$ uses a list of probabilities and a 22\% looked for syndromes. To differentiate between diseases included in a syndrome, $70 \%$ looked for key signs or symptoms.

- When we asked about Diagnostic Error, 65\% thought they were preventable, but 34\% of the respondents thought they could be reduced with more resources.

- When we asked about clinical reasoning, 55\% thought it was a mental process, but 35\% of respondents confuse it with their own clinical practice.

Discussion: We believe that, because the sampling method is open, a selection bias can affect our results, but the number of respondents allowed us to get information to direct teaching to improve the diagnostic process.

Significance: Residents included in this sample, use three clinical reasoning strategies:

1) comparison with mental schemes, 2) assessment of the probability of the entities and 3) to classify the information in syndromes. Teaching how to build mental schemas, the cognitive biases and how to select key symptoms in a syndrome, are the practical conclusion of our survey.

\section{What is your strategy to do the Differential Diagnosis?}

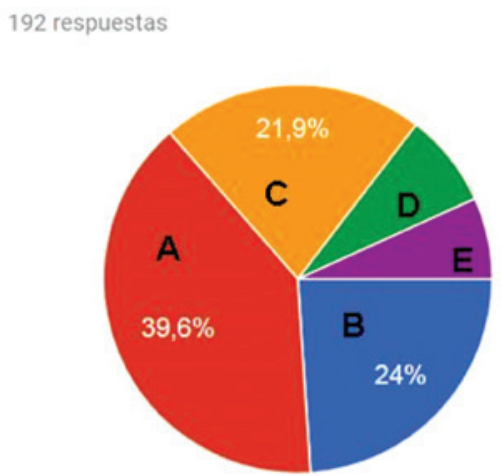
A. I compare with my mental schemes
B. I make a list according to probabilities
C. I organize the information in syndromes
D. I follow an algorithm
E. I follow my intuition 


\title{
Assessment of Reasoning Tool for Chronic Conditions: Pilot Study to Evaluate a Tool to Assess Students' Clinical Reasoning for Chronic Health Conditions
}

\author{
L. Cappiello $^{1}$, D. Pelletier ${ }^{1}$, D. Sharp ${ }^{1}$, G. Harkless ${ }^{1}$ and A. Olsen \\ ${ }^{1}$ University of New Hampshire. \\ ${ }^{2}$ University of Minnesota Medical School
}

\begin{abstract}
As faculty who teach nurse practitioner and medical students, we are interested in promoting the development of expertise in clinical reasoning in our students. We have integrated illness scripts and the Assessment of Reasoning Tool (ART) into our curriculum as measures to build and assess diagnostic reasoning through case presentations. ART has been used for competency-based assessment and has shown to be an effective tool for faculty to use when providing individual feedback to students (Thammasitboon et al., 2018).

The ART was developed to assess clinical reasoning during the presentation of acute health problems (Thammasitboon et al., 2018) but frequently students in primary care settings are providing chronic condition management. In assessing these students, faculty found that elements of the ART tool are not applicable and do not capture important aspects of clinical reasoning for chronic health conditions. It is important for clinicians' training to reflect the relative frequency of conditions that they will see in practice. Primary care clinicians need to gain expertise in the management of chronic physical and mental health problems during training.

The purpose of our study is to design and evaluate a tool to assess students' clinical reasoning for chronic health conditions. The ARTC2 is adapted from the ART (Thammasitboon et al., 2018) and will specifically assess student clinical reasoning during primary care visits for chronic health conditions. The ARTC2 will assess the student's ability to engage in efficient, directed data collection, appropriate use of evidence based clinical guidelines, address the patient's social determinants of health.

In this pilot study, faculty and preceptors will use the ARTC2 to assess the student's ability to formulate a case presentation and collaborate with the patient to devise a mutually agreed upon plan of care. Qualitative data will be gathered from interviews with nursing and medical faculty and primary care preceptors using ARTC2 to evaluate students. Preliminary findings will be reported at the conference.

Thammasitboon, S., Rencic, J.J., Trowbridge, R.L., Olson, A.P.J., Sur, M., Dhaliwal, G. (2018). The Assessment of Reasoning Tool (ART): Structuring the conversation between teachers and learners. Diagnosis (Berl). 5(4):197-203. doi: 10.1515/dx-2018-0052. https://www.ncbi.nlm.nih. gov/pubmed/30407911
\end{abstract}

Assessment of Reasoning Tool for Chronic Health Conditions

\begin{tabular}{llll}
\hline Did the Learner... & Assessment & & Complete \\
\cline { 2 - 4 } & Minimal & Partial & Followed clear line of inquiry, \\
\hline Collect/report history and & Asked questions without clear & Questioning and exam generally & Folic \\
examination data addressing & focus on current chronic health & reflective of current chronic & directing questioning and exam \\
the immediate health concerns & conditions or potential issues. & health conditions, but some & to specific findings. \\
of the patient/family. & Did not elicited patient concern & less relevant or tangential & Elicited patient concern for \\
& for visit/complaint. & questions. & visit/complaint. \\
& Rushed or used time & Elicited patient concern for & Used time efficiently. \\
& inefficiently. & visit/complaint. &
\end{tabular}

Articulate an accurate representation of patient problems using descriptive medical terminology?

Direct evaluation/treatment towards high quality, cost effective care?

\begin{abstract}
Included extraneous information. Missed key findings. Did not translate findings into medical terminology. Did not articulate patient selfmanagement strategies.
\end{abstract}

Did not direct evaluation and treatment toward high quality, cost effective care.
Generally used time efficiently. Generally included key clinical findings (both positive and negative) but either missed some key findings or missed important descriptive medical terminology.

Articulated patient selfmanagement strategies.

Generally directed evaluation and treatment towards high quality, cost effective care considering financial implications, access to care, and patient acceptability.
Gave clear synopsis of clinical problem.

Emphasized important positive and negative findings using descriptive medical terminology.

Articulated patient strengths and barriers for selfmanagement strategies. Directed evaluation and treatment towards high quality, cost effective care considering financial implications, access to care, and patient acceptability 


\begin{tabular}{|c|c|c|c|}
\hline \multirow[t]{2}{*}{ Did the Learner... } & \multicolumn{3}{|l|}{ Assessment } \\
\hline & Minimal & Partial & Complete \\
\hline $\begin{array}{l}\text { Demonstrate patient-centered } \\
\text { care and use of best practice } \\
\text { guidelines? }\end{array}$ & $\begin{array}{l}\text { Did not consider the } \\
\text { established practice guidelines. } \\
\text { Lack of care coordination and } \\
\text { planned care. } \\
\text { Lack of active follow-up to } \\
\text { ensure the best outcomes } \\
\text { Patient/family not involved in } \\
\text { informed decision making. }\end{array}$ & $\begin{array}{l}\text { Generally considered } \\
\text { established practice guidelines. } \\
\text { Some coordination of care } \\
\text { noted. } \\
\text { Appropriate active follow-up. } \\
\text { Patient/family involved in some } \\
\text { aspects of informed decision } \\
\text { making. }\end{array}$ & $\begin{array}{l}\text { Demonstrated alignment of } \\
\text { patient goals and best practice } \\
\text { guidelines. } \\
\text { Coordinated, follow-up plan in } \\
\text { place. } \\
\text { Care is customized and reflects } \\
\text { patient/family needs, values } \\
\text { and choices. }\end{array}$ \\
\hline $\begin{array}{l}\text { Demonstrate the ability to } \\
\text { think about their own thinking } \\
\text { (metacognition)? } \\
\text { Consider asking: Is there } \\
\text { anything about the way you are } \\
\text { thinking or feeling about this } \\
\text { case that may lead to error? }\end{array}$ & $\begin{array}{l}\text { Not able to describe the } \\
\text { influence of cognitive } \\
\text { tendencies of emotional/ } \\
\text { situational factors that may } \\
\text { have influenced decision- } \\
\text { making. }\end{array}$ & \multicolumn{2}{|c|}{$\begin{array}{l}\text { Can name one cognitive tendency or emotional/situational factor } \\
\text { that may have influenced decision-making. }\end{array}$} \\
\hline Comments: & & & \\
\hline OVERALL ASSESSMENT & NEEDS IMPROVEMENT $\square$ & MEETS COMPETENCY $\square$ & EXCELLENCE $\square$ \\
\hline
\end{tabular}

Adapted from SIDM ART Tool by Cappiello \& Pelletier (2019)

\section{Use of Chart-Stimulated Recall as an Educational Tool to Explore Uncertainty in Medical Decision Making Among Senior Internal Medicine Residents}

M. Mutter, E. Yecies and D. DiNardo

University of Pittsburgh School of Medicine

Purpose/Problem: Errors in medical decision making have been linked to adverse events and patient harm, particularly when diagnostic or therapeutic uncertainty exists. Guided reflection has been identified as a promising tool for improving diagnostic decision making, including the approach to uncertainty. Therefore, we sought to determine whether reflection about a patient case with another physician through ChartStimulated Recall (CSR, a method that pairs patient chart review with an oral interview component) is a valuable educational tool to promote reflection about uncertainty in medical decision making.

Description of program, assessment, or study: We designed and implemented a CSR-based reflection exercise for senior internal medicine (IM) residents on night float rotations at the University of Pittsburgh Medical Center. We first developed a CSR interview guide and then conducted pilot interviews with residents to further refine the interview guide and reflection questions. We then implemented the reflection exercise with all PGY-2 and PGY-3 IM residents on a night float rotation over a 6-month period; each resident participated in one session. For the exercise, participants selected one new patient admission in which there was uncertainty in medical decision making. Faculty interviewers then guided each resident through a reflection exercise to explore the nature of, contributors to, and sequelae of the uncertainty in each case. In order to evaluate the intervention, participants completed a survey after the reflection exercise.

Outcomes: We implemented this CSR exercise in February 2019; twenty-four residents have participated in the reflection exercise so far. Preliminary feedback from participants suggests that reflection on the decision-making process is a valuable exercise. Suggestions for improvement included consideration of a change of time for the exercise, and the provision of feedback, among others.

Discussion: Reflection is an important method to improve the decision-making process, which can ultimately lead to improved patient care. Our study on chart-stimulated recall as a means of reflection noted both benefits of and challenges to such an intervention. Implementation of such an exercise should consider the optimal timing of a reflection exercise, as well as the opportunity to provide residents with feedback on their decision-making process.

Significance of Findings: Given the frequency and potential harm of errors in medical decision making, interventions aimed at improving decision making are important. CSR is a valuable educational tool that can be used to guide reflection on uncertainty in medical decision making in internal medicine residents, with a goal of improving diagnostic reasoning skills. 


\title{
Self-Assessment of Diagnostic Reasoning in Nurse Practitioner Education: Longitudinal Perceptions of Developing Competencies
}

\author{
S. K. Smith
}

University of Minnesota, School of Nursing

Purpose: The purpose of this initiative was to examine one approach to evaluating the effectiveness of a longitudinal diagnostic reasoning curriculum in Nurse Practitioner (NP) education, by assessing NP students' formative and summative perceptions of their evolving diagnostic reasoning competencies using the Self-Assessment of Diagnostic Reasoning tool. This pilot project presents an adaptation of the Assessment of Reasoning Tool (SIDM, 2019), to begin inquiry into the effectiveness of a new diagnostic reasoning education program for Nurse Practitioner education.

Description of the program: As part of a larger initiative to introduce the teaching of diagnostic reasoning into Nurse Practitioner education, students in an Adult-Gerontology Nurse Practitioner (AGNP) Doctor of Nursing Practice (DNP) program in the upper Midwest were provided with an array of diagnostic reasoning didactic content and assignments over four semesters within their clinical practicum courses. The Assessment of Reasoning Tool (SIDM, 2019) was used to develop the Self-Assessment of Diagnostic Reasoning, a formative and summative self-evaluation of students' evolving diagnostic reasoning knowledge and skills. Students were provided with a longitudinal curriculum incorporating an array of contemporary diagnostic reasoning didactic content, readings, individual and group exercises, and clinical assignments. Over their four semesters of supervised clinical practice, students were required to complete the Self-Assessment of Diagnostic Reasoning twice per semester. A final measure compares students', preceptors', and the supervising clinical faculty member's evaluation of students' performance. Outcomes: Data will be analyzed for changes over time, from the beginning to near the end of the students' clinical rotations. Each student's final self-assessment will be compared with their clinical preceptor's and supervising faculty member's evaluation on the same items. Outcomes will demonstrate students' evolution of their self-assessments over time as compared to the evaluations of their clinical preceptors and supervising faculty.

Discussion: The explicit teaching of diagnostic reasoning as an essential practice competency is being newly introduced to NP education in this AGNP program, with curricular innovations that include SIDM and other contemporary diagnostic reasoning research and scholarship. The development of assessment tools to evaluate students' performance in diagnostic reasoning is a necessary component of curriculum development. Self-assessment provides a supportive and non-threatening approach to learning about how to assess the development of diagnostic reasoning competencies.

Significance of findings: This application of the Assessment of Reasoning Tool provides an effective approach to introducing and evaluating the effectiveness of a longitudinal diagnostic reasoning curriculum in Nurse Practitioner education.

\section{Practice Improvement}

\section{Providing Feedback about Diagnosis to Calibrate How Clinicians Think in a Learning Health Care System}

\author{
$\underline{\text { A. Meyer }}^{1}$, D. Upadhyay ${ }^{2}$, D. Torretti², C. A. Collins ${ }^{2}$, M. H. Fitzpatrick ${ }^{2}$ and H. Singh ${ }^{1}$ \\ ${ }^{1}$ VA Center for Innovations in Quality, Effectiveness and Safety and Baylor College of Medicine \\ ${ }^{2}$ Geisinger
}

Statement of the problem: Reducing harm from diagnostic error requires approaches that seek to improve systems issues and clinician decision-making. Diagnostic errors often involve suboptimal analytical thinking, or erroneous or biased intuitive decision-making that can be averted by well-calibrated clinicians (clinicians whose confidence in their diagnostic accuracy aligns with actual accuracy). Research from outside healthcare suggests calibration can be achieved through consistent learning about one's performance through feedback. However, very little feedback occurs once clinicians enter practice. Learning health systems need to implement policies and procedures to provide this feedback, yet little is known about how to do so.

Description of the program: Partnering with a large healthcare organization, we are developing elements of a learning health system to measure and improve diagnostic safety in clinical practice. Important steps of this process include gathering actionable data on potential diagnostic errors (e.g., via institutional safety data, clinician reports, electronic triggers, and patient reports), identifying potential learning opportunities, then providing feedback to involved clinicians and others for broader learning.

Feedback facilitators (department leaders) are first trained to deliver feedback in a structured, supportive, non-punitive way. They are given a guide, laying out recommendations for successful feedback sessions (e.g., scheduling sessions in a timely manner, allowing clinicians time to review cases beforehand, creating a learning environment, and identifying positive aspects of case evaluation/management and key issues/ learning points).

Following the sessions, feedback facilitators and recipients are surveyed about their experiences and perceptions regarding usefulness of the feedback sessions for improving future diagnosis and patient safety. 
Findings to date: Thus far, feedback sessions have been conducted on 11 diagnostic error cases from emergency medicine, primary care, and hospital medicine. Feedback facilitators $(n=12)$ and recipients $(n=10)$ answered post-feedback session surveys and found these sessions useful. Feedback facilitators reported feeling feedback recipients were receptive to learning and discussing potential missed opportunities in diagnosis. Feedback recipients reported feeling conversations were constructive and non-punitive and would allow them to take concrete steps toward improving diagnosis. Both groups felt discussions would improve the safety of patients in clinical practice. We are using these experiences to refine and test the process, so it can be exported to other departments/healthcare institutions.

Lessons learned: We are developing a learning healthcare system for diagnosis that includes feedback to enable learning and improvement from missed opportunities in diagnosis. Future research and implementation efforts should assess long-term effects of this feedback on diagnostic performance and safety.

\title{
Analyzing Diagnostic Error to Create More Secure Practice Atmosphere for Young Doctors
}

\section{K. Nakano}

Shonan Fujisawa Tokushukai Hospital

Statement of problem: In Japan, PGY 1s and 2s traditionally are the busiest spending much of their time examining patients, studying, and doing night shifts. Having less experience in medicine diagnostic error tends to occur when they are in the front line of medicine. The actual practitioner must be pre-cautious of when and where diagnostic errors can happen, but it is difficult for young doctors.

Description of the intervention or program: Using questionnaire based on diagnosis error evaluation and research (DEER) taxonomy by GD Schiff, et al. PGY 1s and 2s analyzed their recent diagnostic error.

Findings to date: 17 residents participated in the questionnaire. "Failure in considering the diagnosis " achieved most votes followed by "Failure in weighing critical piece of history" "Failure/delay in eliciting critical physical exam finding" "failure in weighing critical piece of physical exam." consecutively. Not many votes went to values that did not involve residents.

Lessons learned: Though the result is center limited, the chosen reasons of error are mainly resident's experience and knowledge dependent. To reduce this type of error, residents must experience more and continuous back up is needed. Also, looking back at some of the cases analyzed show that there are unvoted causes of errors and they are usually causes coming aside from the residents. These errors are usually system errors that senior doctors and other staff members need to recognize and improve. This shows that reasons of errors differ between the actual practitioner and the outsider and that it's hard for the actual practitioner to recognize errors that don't concern him/her. Therefore, senior doctors need to educate and improve both the apprentice and the surroundings to reduce diagnostic errors.

\section{The Cognitive Bias Think Tank - Mitigating the Influence of Cognitive Biases on Diagnostic Error}

\author{
U. Nawab, A. Mack, K. Shaw, T. Garner and A. Colfer
}

Children's Hospital of Philadelphia

Purpose/Problem: Diagnostic error, including misdiagnosis and delay in diagnosis/treatment, is a significant patient safety concern resulting from a large number of potential causes. Similarly, clinical decision making is a complex mental process affected by a myriad of contexts. Cognitive bias is one key component underlying diagnostic error, but identifying, discussing, and mitigating bias in the setting of diagnostic error is challenging. Challenges include a lack of a framework for "thinking about thinking” as well as the affirmative need for "psychological safety." Description of intervention: The aim was to increase discussion of cognitive bias in a more robust and meaningful way and to develop local expertise on cognitive bias and mitigation strategies. Children's Hospital of Philadelphia is a 562-bed quaternary children's hospital with $\sim 30$ medical/surgical divisions. A multidisciplinary team, named the Cognitive Bias Think Tank (CBTT), assessed whether diagnostic error and cognitive bias were discussed within divisions. To increase discussions at local morbidity and mortality (M\&M) or Patient Case conferences, the CBTT developed a 4-part targeted strategy for physicians which included (1) an education session for M\&M organizers on diagnostic error/cognitive bias, (2) development of a M\&M slide template to foster discussion, (3) hospital-wide M\&Ms highlighting cognitive bias and recent patient cases, and (4) cognitive bias presentations at division meetings. The objective was to infuse local M\&Ms with concepts of cognitive bias identification and potential de-biasing strategies, allowing clinicians to continue to refine clinical practice for safer care.

Findings to date: 23 medical/surgical divisions responded to the initial survey in March 2018 indicating review of patients or safety events in the setting of M\&M or Patient Case Conferences. 91\% discussed diagnostic error; however, 32\% did not consider cognitive bias as it relates to diagnostic error. Barriers included lack of knowledge, fear of blame, and competing priorities. In a post-intervention survey in June 2019, $95 \%$ of the divisions discussed diagnostic error and $90 \%$ were examining cognitive bias in relation to diagnostic error, demonstrating a $22 \%$ increase with a 2 -fold increase in mitigation discussions. $80 \%$ of divisions utilizing the templates describe enhanced, more organic conversation around cognitive bias in conferences and on rounds/signout. 
Lessons learned: A systematic approach to cognitive bias requires "buy in" from physician stakeholders. Multiple approaches are needed to address cognitive bias and diagnostic error, this is one step in advancing that effort. Next steps include understanding competing priorities and improving the psychological safety to decrease the fear of blame.

\title{
Patient and Provider Focused Information to Improve Diagnostic Accuracy of Clinical Laboratory Test Information
}

\author{
$\underline{\text { C. N. Otto }}^{1}$ and K. Golemboski ${ }^{2}$ \\ ${ }^{1}$ Rutgers University \\ ${ }^{2}$ Bellarmine University
}

Statement of Problem: Many factors influence usefulness of clinical laboratory tests, including specimen quality and interpretation of results. Patients must be adequately prepared prior to specimen collection, and providers must be informed to appropriately interpret test results. Providers frequently order laboratory tests for their patients without sharing any additional information other than the name of the laboratory test to be performed.

Description of the intervention or program: The American Society for Clinical Laboratory Science (ASCLS) Patient Safety Committee has developed a series of informational products (Patient Safety Tips) to provide patients with information about how to prepare for specimen collection to ensure that the specimen collected provides the best information about the patient's health status. Patient Safety Tips have also been created for providers to help them to order and interpret laboratory test results. Examples of Patient Safety Tips include: Blood Specimen Collection; Common Substances that Affect Laboratory Test Results; Fasting: What is it? Does it matter?; and Drug-related Laboratory Test Interference. Findings to date: A recent survey of ASCLS membership indicated that 35\% of members are aware of the availability of Patient Safety Tips on the ASCLS website and have used them in some manner; $17 \%$ are aware of the Tips, yet have not used or shared them; and $48 \%$ of members are not aware of the availability of Patient Safety Tips. Tips have been shared with friends and family, distributed at health, career and science fairs, shared with patients who use laboratory services, and distributed to providers. Educators have shared Patient Safety Tips with their students as well as with colleagues in other health professions educational programs. Of those who have used Patient Safety Tips, 22\% found them to be extremely useful, $45 \%$ to be very useful and 33\% to be somewhat useful.

Lessons learned: Patient Safety Tips are useful tools to provide vital information about clinical laboratory testing for patients and providers; however, to improve the quality of laboratory test information, medical laboratory professionals need to share the Tips with patients and providers. Additionally, when medical laboratory professionals share the Patient Safety Tips with providers, those providers then need to share appropriate Tips with patients. Information about clinical laboratory testing is key to improving the quality of diagnostic accuracy.

\section{High Volume of MRIs at End of Year: An Exploration of Causative Factors}

\author{
M. Pitot ${ }^{1}$, C. Chin ${ }^{1}$, A. Johri ${ }^{1}$, E. Jones ${ }^{1}$, M. Bruno ${ }^{2}$ and S. Hardy ${ }^{2}$ \\ ${ }^{1}$ Penn State College of Medicine \\ ${ }^{2}$ Hershey Medical Center
}

Statement of Problem: At Hershey Medical Center (HMC), a retrospective analysis of MRI volumes from 2018-2019 revealed an upward trend in studies throughout the year, peaking in November and December. This bolus has the potential to increase physician fatigue, medical errors, technologist overtime, and medical costs.

Description of Intervention or Program: In order to develop a more targeted plan to better distribute this MRI volume throughout the year, we created a fishbone diagram to examine all potential contributors to the problem.

The three major "bones" of the diagram were: the patients receiving MRIs, the institution ordering and supplying the MRIs, and the insurance companies providing reimbursement for the MRIs. By examining each category individually, we could then assess whether each was a significant contributor to the MRI bolus.

While literature that specifically examined patient motivators for MRI utilization was lacking, we proposed that variability in patient scheduling could result from cost fluctuations, concern about health, physician recommendations, time constraints, and appointment availability. Institutional variability could result from referring physician patterns of ordering, inefficient MRI scheduling, and temporal variance of order indication (ie, if increased ligamentous injuries during the winter necessitate more MRIs). Insurance factors, however, seemed to be the predominant driver of MRI scheduling; a review of the literature revealed that patient clinic visits significantly increased once patients met their deductible, something than could also lead to increased MRIs at the end of the year2,3,4.

Findings to Date: Following development of this fishbone analysis, solutions were then proposed for each "bone." These included incentivebased programs for MRI scheduling at particular times during the year, expiration dates for MRI orders, and non-uniform deductible dates. Lessons Learned: Although currently lacking hard data, this project has many clinical implications; most significantly, it aims to analyze the root cause of and address solutions for the puzzling MRI bolus seen at the end of the year at HMC, which has significant effects at the patient, clinician, and institution level in terms of preventing physician burnout, reducing healthcare costs, and ultimately improving patient safety. 
Proposed Solutions

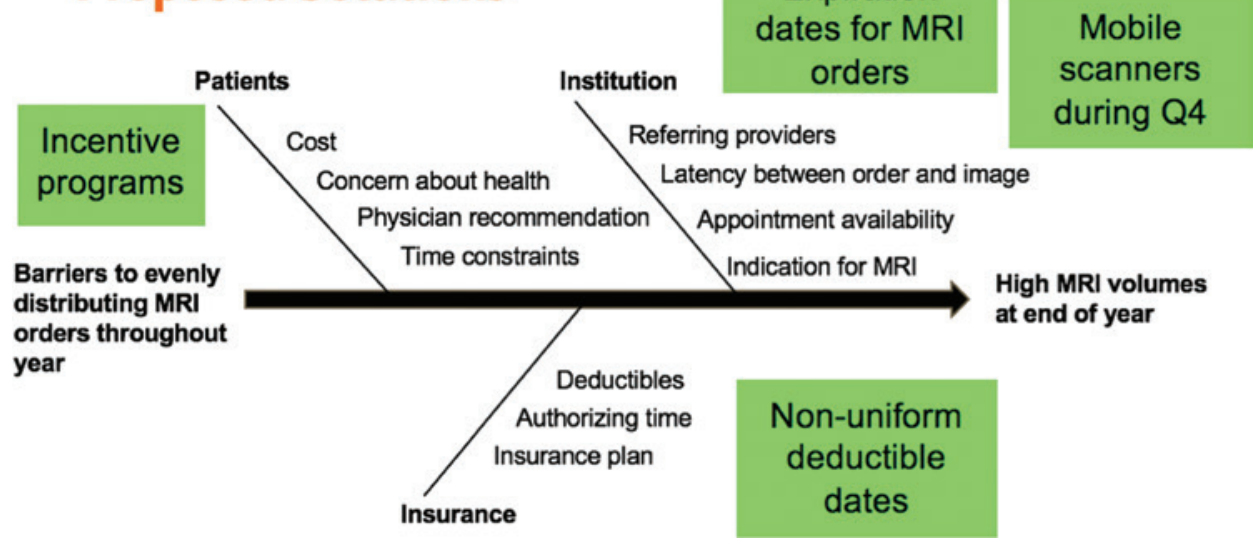

\section{Interstellar: Improving Diagnostic Decision Making through Virtual Communication}

J. Welkie, K. Toth, P. Hong and M. Bruno

Penn State College of Medicine

Diagnostic errors are a common and costly problem affecting all fields of medicine. Within diagnostic radiology, a particular vulnerability for diagnostic error occurs when imaging studies (e.g. CT, MRI) performed at one institution are reinterpreted in a second facility. This is common when patients are transferred between hospitals, often from a community hospital to an academic or regional medical center for a higher level of care. In such situations, discrepancies among primary and secondary diagnostic reports occur at an astonishing frequency, 32\% in one published series, with nearly $20 \%$ of discrepancies indicating change in medical management. Most settings lack a standardized way for radiologists to report or communicate these discrepancies and rapidly detect diagnostic errors that occur when patients are transferred between institutions. Lack of communication channels between the primary and secondary interpreters creates a missed opportunity for discussion of discrepant reports, peer learning, and subsequent reduction in diagnostic errors.

Our three-part intervention will: (1) improve identification of these discrepancies, (2) create a reporting system, and (3) foster discussion of discrepant reports between radiologists at multiple institutions within the local community. The first two components center on identifying and compiling discrepant reports for future discussion. The main component of the intervention, modeled after the highly successful Intergalactic Conference, is a monthly "Interstellar" Radiology Teleconference, which will provide a platform for open discussion and peer learning between radiologists at different institutions. Developing a consistent space where radiologists are able to discuss local diagnostic errors in a safe environment will foster an atmosphere of learning and collegiality. We propose that this sense of community will lead to a reduction in diagnostic error, provide a platform for effective feedback, and help prevent physician burnout.

In moving forward with our intervention, identifying discrepant cases proved to be difficult as primary reports were inconsistently uploaded into our system. Thus, in order to begin identifying discrepant reports, we will have primary reports from outside institutions transferred along with the imaging. Once identified, cases can be flagged for future discussion using Primordial ${ }^{\mathrm{TM}}$ radiology software or a secure excel file. Ideally, the Interstellar conferences will begin within the year via Zoom, a free and secure teleconference technology. Efficacy will be evaluated using measures of participation and participant self-reports of performance improvement. Eventually, our intervention may be replicated in other institutions seeking to develop a similar system of identifying, reporting, and discussing diagnostic discrepancies among all fields of medicine. 


\title{
Poster Session 2 \\ Monday, November 11 \\ 4:15 PM - 5:15 PM
}

\section{Scientific}

\section{Clinical Reasoning Errors in Hospital Medicine Physicians}

\author{
$\underline{\text { G. Astik }}^{1}$, A. Olson ${ }^{2}$ and K. O’Leary ${ }^{1}$ \\ ${ }^{1}$ Northwestern University \\ ${ }^{2}$ University of Minnesota
}

Background: Clinical reasoning errors are common in medicine. One of the challenges to improving clinicians' clinical reasoning skills is the absence of feedback. The identification of diagnostic error is often delayed, typically occurring only after the patient has experienced harm. I endeavor to solve this problem. Care transitions are inherent to the practice of hospital medicine and although care transitions have long been recognized as an area of vulnerability for hospitalized patients, they also represent opportunities for error recognition and improvement as the oncoming clinician reviews and revises the plan established by the prior clinician. Our study is unique because we are capitalizing on the inherent care transitions in hospital medicine as opportunities to evaluate the care provided by clinicians, look for errors and also provide meaningful feedback. We hypothesize that care transitions can provide an opportunity to learn about clinical decisions and provide feedback to clinicians. Methods: We created a questionnaire based on prior research in this area to assess changes in diagnoses, diagnostic testing and treatment plan and whether those changes were due to disagreement or other factors (e.g. disease evolution or diagnostic refinement). We identify $2-3$ patients during each transition from one hospitalist to another and administer the survey to the hospitalist assuming care from the former provider. From this report, patients will be added to a protected questionnaire by a team member who has access will be able to assign patients and questionnaires to the correct physician.

Completed questionnaires, in which there was a change in diagnosis or therapeutic decisions, will be reviewed by one of two physician coinvestigators. These co-investigators evaluate whether there was an error. Using these reviews, we will establish the frequency of errors in order to ascertain our baseline performance data. It is imperative to identify these errors or changes and leverage the opportunity to adjudicate clinical decisions.

Results: My study is currently ongoing with a survey response rate of above $90 \%$. I will have preliminary results available by the time of the conference this fall.

Conclusion: Diagnostic errors are a major contributor to malpractice claims and we feel that, by recognizing clinical reasoning errors and providing feedback to physicians, the number of errors and claims would decrease. We hope to set a benchmark on the baseline rate of clinical reasoning errors.

\section{What is the Accuracy of Chest X-ray in Lung Cancer Diagnosis?}

\author{
$\underline{\text { S. H. Bradley }}{ }^{1}$, M. Kennedy ${ }^{2}$, M. Callister ${ }^{2}$, W. Hamilton ${ }^{3}$, B. Shinkins ${ }^{1}$ and R. Neal ${ }^{1}$ \\ ${ }^{1}$ University of Leeds \\ ${ }^{2}$ Leeds Teaching Hospitals NHS Trust \\ ${ }^{3}$ University of Exeter
}

Background: Although CT is increasingly used for lung cancer detection, the availability and low cost of chest x-ray (CXR) means it remains the first line test in the UK and in rural settings in the US. There is remarkably little evidence for the accuracy of CXR in the detection of symptomatic lung cancer. A systematic review has reported that CXR has a sensitivity of $77-80 \%$ for lung cancer in symptomatic patients, but this estimate relied on only three studies with a total population of 480 .

Methods: All patients diagnosed with lung cancer in Leeds Teaching Hospitals NHS Trust between 2008 and 2013 who had a CXR requested by their family physician in the year before diagnosis were categorised based on the first CXR report:

1 - suspicious for cancer, urgent follow up recommended

2 - abnormal, routine follow up recommended (e.g. repeat CXR after interval)

3 - abnormal, but not suspicious for cancer

4 - normal 
Categories 1 \& 2 were considered 'positive' and categories 3 \& 4 'negative'. Analysis was conducted with respect to category of first CXR performed (index CXR), time from index CXR to diagnosis and stage at diagnosis.

Results: 1559 (44.0\%) had at least one CXR in the year before diagnosis. The index CXR was 'positive' in 1312 (84.2\%), with 1025 (65.7\%) categorised as '1' and $287(18.4 \%)$ as '2'. 247 (15.8\%) were 'negative', of which 152 (9.7\%) were ' 3 ' and 95 (6.1\%) were 4. Median time from index CXR to diagnosis was 44 days for those with a 'positive' result compared to 190 days to those who had a 'negative' CXR. Within the 'positive' group, those who had an index CXR categorised as '2' had a median time to diagnosis of 91 days, compared to 37 days for those categorised as ' 1 '. However stage at diagnosis was similar across groups.

Conclusions: This is by far the largest such investigation and brings new understanding to the limitations of CXR in lung cancer detection. Though the sensitivity is higher than reported in in smaller studies, the failure of CXR to detect cancer in 16\% represents an important cause of diagnostic error. 'False negative' results and abnormal reports which recommended routine rather than urgent follow up are associated with significant diagnostic delay. Research on the comparative diagnostic accuracy of CXR and CT is urgently required. The results of this study will be vital for the trial design of such research.

What is the accuracy of chest x-ray in lung cancer diagnosis?

\begin{tabular}{|c|c|c|c|c|c|c|c|}
\hline & $\begin{array}{r}\text { Total patients } \\
\text { diagnosed } \\
\text { lung cancer } \\
2008-2013\end{array}$ & $\begin{array}{r}\text { Index CXR = 1 } \\
\text { (suspicious) }\end{array}$ & $\begin{array}{r}\text { Index CXR = } \\
2 \text { (abnormal, } \\
\text { routine follow } \\
\text { up) }\end{array}$ & $\begin{array}{r}\text { Index CXR = } 3 \\
\text { (abnormal, no } \\
\text { follow up) }\end{array}$ & $\begin{array}{r}\text { Index } \\
\text { CXR }=4 \\
\text { (normal) }\end{array}$ & $\begin{array}{r}\text { 'Positive' } \\
\text { (Index CXR }=1 \\
\text { or } 2 \text { ) }\end{array}$ & $\begin{array}{r}\text { 'Negative' } \\
\text { (Index CXR } \\
=3 \text { or } 4 \text { ) }\end{array}$ \\
\hline Number & 1559 & $1025(65.7 \%)$ & 287 (18.4\%) & $152(9.7 \%)$ & $95(6.1 \%)$ & $1312(84.2 \%)$ & 247 (15.8\%) \\
\hline Age Range (years) & $30-95$ & $33-95$ & $30-94$ & $48-93$ & $36-90$ & $30-95$ & 36-93 \\
\hline Mean age & 71 & 71 & 72 & 74 & 70 & 71 & 73 \\
\hline Male & $843(54.1 \%)$ & $557(54.3 \%)$ & $148(59.9 \%)$ & $88(57.9 \%)$ & $50(52.6 \%)$ & $705(53.7 \%)$ & $138(55.9 \%)$ \\
\hline Female & $716(45.9 \%)$ & $468(45.6 \%)$ & $139(48.4 \%)$ & $64(42.1 \%)$ & $45(47.4 \%)$ & $607(46.3 \%)$ & $109(44.1 \%)$ \\
\hline Median days to Diagnosis & 113.0 & 37.0 & 91.0 & 189.5 & 196.0 & 44.0 & 190.0 \\
\hline Stage I or II at diagnosis & $444(28.5 \%)$ & $282(27.5 \%)$ & $85(29.6 \%)$ & $49(32.2 \%)$ & $28(29.5 \%)$ & $367(28.0 \%)$ & 77 (31.2\%) \\
\hline Stage III or IV at diagnosis & $1111(71.3 \%)$ & $739(72.1 \%)$ & $202(70.4 \%)$ & $103(67.8 \%)$ & $67(70.5 \%)$ & $941(71.7 \%)$ & $170(68.8 \%)$ \\
\hline Unknown stage & $4(0.4 \%)$ & $4(0.4 \%)$ & $0(0.0 \%)$ & $0(0.0 \%)$ & $0(0.0 \%)$ & $4(0.4 \%)$ & $0(0.0 \%)$ \\
\hline
\end{tabular}

\section{Updates to Referring Clinicians Regarding Critically Ill Children Admitted to the Pediatric Intensive Care Unit: A State- Wide Survey}

$\underline{\text { C. L. Cifra }}^{1}$, C. R. Tigges ${ }^{1}$, S. L. Miller ${ }^{1}$, L. A. Herwaldt ${ }^{1}$ and H. Singh ${ }^{2}$

${ }^{1}$ University of Iowa Carver College of Medicine

${ }^{2}$ Baylor College of Medicine, Michael E. DeBakey VA Medical Center

Background: Front-line community providers are expected to make accurate and timely diagnostic and therapeutic decisions before transferring patients to the pediatric intensive care unit (PICU). However, they do not always receive feedback regarding their diagnoses or the outcomes of their decisions. Timely updates from the PICU to referring clinicians, which include patients' diagnoses, treatment, and clinical course, can ultimately improve outcomes of critically ill children. As the first step in developing a formal system to provide referring clinicians with timely updates about their PICU patient's outcomes, we sought to determine the characteristics of existing patient updates received by referring clinicians. We also aimed to determine clinician preferences regarding content, delivery, and timing of updates.

Methods: We performed a cross-sectional study using a web-based survey administered to clinicians practicing in emergency departments (EDs), inpatient units, and outpatient clinics in Iowa who billed for $=5$ pediatric patients or referred $=1$ patient to the University of Iowa (UI) PICU in the year before survey administration. The survey was developed iteratively with input from pediatric stakeholders and piloted on adult ED physicians. Descriptive statistics were reported and responses between non-UI and UI clinicians were compared.

Results: One hundred and one clinicians responded (51 non-UI, 50 UI-affiliated). Each clinician saw an average of 52\% pediatric patients over one year, of which $8 \%$ required PICU admission. Clinicians received updates on $40 \%$ of referred patients. Seventy percent of UI clinicians obtained updates via self-initiated electronic record review while $37 \%$ of non-UI clinicians relied on PICU communication ( $p=0.013$ ). Clinicians preferred to receive updates via email (55\%) while the patient was still in the PICU (60\%). Updates containing patient diagnoses, procedures performed, and clinical outcomes were preferred. However, $5 \%$ of clinicians would prefer not to receive PICU updates at all. Among clinicians who received updates, $13 \%$ received unexpected information; $40 \%$ changed their practice as a result of the feedback.

Conclusions: Referring clinicians received updates on only $40 \%$ of referred PICU patients, although several updates influenced clinical practice. Our findings will inform development of a formal feedback system between the PICU and referring clinicians/institutions. 
Table: Characteristics of Pediatric Intensive Care Unit Updates to Referring Clinicians and Clinicians' Preferences for Receiving Updates

\begin{tabular}{|c|c|c|c|}
\hline \multirow[t]{2}{*}{ Characteristics of Updates $^{a}$} & \multicolumn{2}{|c|}{ Referring Clinician Affiliation ${ }^{b}$} & \multirow[t]{2}{*}{$p$ value } \\
\hline & $\begin{array}{r}\text { Non-UI Clinician } \\
n=51\end{array}$ & $\begin{array}{r}\text { UI Clinician } \\
n=50\end{array}$ & \\
\hline \multicolumn{4}{|l|}{ Characteristics of PICU Updates } \\
\hline Clinicians who received any PICU update for referred patients in the past 12 months, $n$ (\%) & $39(76)$ & $37(74)$ & 1.000 \\
\hline $\begin{array}{l}\text { Proportion of patients referred to any PICU in the past } 12 \text { months for whom updates were } \\
\text { received, mean \% }(\mathrm{sd})\end{array}$ & $41.4(41.0)$ & $38.5(44.0)$ & 0.925 \\
\hline \multicolumn{4}{|l|}{ Most common way of receiving PICU updates, $\mathrm{n}(\%)$} \\
\hline Referring clinician was contacted by the PICU & $11(37)$ & $1(3)$ & 0.013 \\
\hline Referring clinician contacted the PICU & $6(20)$ & $5(15)$ & \\
\hline Updates received via a third party without PICU contact & $3(10)$ & $1(3)$ & \\
\hline From electronic medical records (for clinicians with access) & $10(33)$ & $23(70)$ & \\
\hline Other (In-person contact, M\&M conference) & 0 & $3(9)$ & \\
\hline \multicolumn{4}{|l|}{ Timing of receiving updates from the PICU, $\mathrm{n}(\%)$} \\
\hline Within 1 day of PICU admission & $3(11)$ & $11(37)$ & 0.055 \\
\hline Within 1 week of PICU admission & $9(32)$ & $5(17)$ & \\
\hline More than 1 week after PICU admission & $2(7)$ & 0 & \\
\hline Within 1 day after PICU discharge & $1(4)$ & $1(3)$ & \\
\hline Within 1 week after PICU discharge & $5(18)$ & $1(3)$ & \\
\hline More than 1 week after PICU discharge & $5(18)$ & $9(30)$ & \\
\hline \multicolumn{4}{|l|}{ Preferences of Referring Clinicians for Receiving Updates } \\
\hline Clinicians who do not prefer to receive PICU updates, n (\%) & $2(5)$ & $3(7)$ & 1.000 \\
\hline \multicolumn{4}{|l|}{ Preferred method of receiving PICU updates, $\mathrm{n}(\%)$} \\
\hline Phone & $8(20)$ & $10(23)$ & 0.004 \\
\hline Email & $17(43)$ & $29(67)$ & \\
\hline Fax & $7(18)$ & $1(2)$ & \\
\hline Mailed letter & $6(15)$ & 0 & \\
\hline \multicolumn{4}{|l|}{ Preferred timing of receiving PICU updates, $\mathrm{n}(\%)$} \\
\hline Within 1 day of PICU admission & $4(11)$ & $12(28)$ & 0.280 \\
\hline Within 1 week of PICU admission & $10(26)$ & $11(25)$ & \\
\hline More than 1 week after PICU admission & $11(29)$ & $12(28)$ & \\
\hline Within 1 day after PICU discharge & $2(5)$ & $2(5)$ & \\
\hline Within 1 week after PICU discharge & $8(21)$ & $4(9)$ & \\
\hline More than 1 week after PICU discharge & $2(5)$ & 0 & \\
\hline \multicolumn{4}{|l|}{$\begin{array}{l}\text { Preferred data elements to be included in PICU updates (most relevant to provider's } \\
\text { practice), } \mathrm{n}(\%)\end{array}$} \\
\hline Current or discharge diagnoses & $33(87)$ & $37(86)$ & 0.830 \\
\hline Procedures/operations undergone & $29(76)$ & $30(70)$ & \\
\hline Length of PICU stay & $13(34)$ & $16(37)$ & \\
\hline Patient outcome (discharge vs. transfer vs. death) & $35(92)$ & $36(84)$ & \\
\hline PICU clinician contact & $14(37)$ & $15(35)$ & \\
\hline
\end{tabular}

aUpdates are defined as any information pertaining to the patient's course, current or discharge diagnosis, and outcomes during their stay in the pediatric intensive care unit.

${ }^{b}$ Affiliation refers to employment by the University of lowa.

‘Groups were compared using Fisher's exact test for categorical variables and Wilcoxon rank-sum test for continuous variables. $\mathrm{UI}$ - University of lowa, PICU - pediatric intensive care unit, M\&M - morbidity and mortality 


\title{
Anxiety Diagnostic Errors in an Integrated Healthcare System
}

\author{
$\underline{\text { T. L. Fletcher }}{ }^{1}$, M. Escamilla ${ }^{1}$, N. E. Hundt ${ }^{1}$, M. E. Kunik ${ }^{1}$, H. Singh ${ }^{2}$ and M. A. Stanley ${ }^{2}$ \\ ${ }^{1}$ Michael E. DeBakey VA Medical Center \\ ${ }^{2}$ Baylor College of Medicine
}

Background: Few studies have investigated diagnostic errors in psychiatric conditions, where accurate diagnostic labels greatly impact case identification and access to appropriate treatments. For instance, 480,663 Veterans enrolled in the Veterans' Healthcare Administration carry a diagnosis of 'unspecified anxiety disorder' but this non-specific psychiatric diagnosis may be a barrier to receiving appropriate mental health services. Even though this is the most common anxiety diagnosis in primary care settings, it carries little clinical utility because treatment guidelines for unspecified anxiety disorder do not exist. In order to lay groundwork for future interventions to improve the accuracy and timeliness of anxiety and trauma-related diagnoses, we sought to examine the types of anxiety diagnostic errors and identify contributory factors.

Method: Study psychologists conducted "gold standard” psychiatric diagnostic interviews with 18 Veterans diagnosed with unspecified anxiety disorder in primary care. A trained research assistant conducted a secondary review of the Veterans' electronic medical records (EMR) using the Safer Dx - Mental Health Instrument to determine the presence/absence of diagnostic errors and to identify diagnostic process breakdowns for each error case.

Results: None of the of the 18 patients met criteria for unspecified anxiety disorder based on the gold standard psychiatric diagnostic interview. However, 4 of the 18 received accurate specific anxiety diagnoses in the EMR within 90 days of the unspecified anxiety diagnosis and were thus deemed non-error cases. The remaining 14 patients $(78 \%)$ were determined to have a diagnostic error. Ten errors were due to use of unspecified anxiety diagnosis when a specific anxiety diagnosis was warranted (missed diagnoses included social anxiety disorder $70 \%$, generalized anxiety disorder 50\%, panic disorder $20 \%$, trichotillomania $20 \%$, obsessive compulsive disorder $10 \%$ ). Four errors were due to use of unspecified anxiety diagnosis when anxiety symptoms were not severe enough to warrant diagnosis. Diagnostic process breakdowns most frequently involved the absence of standardized diagnostic assessments (93\%) and anxiety symptoms not being documented and/or acted upon during the patient-provider encounter (86\%), but were also related to follow-up and tracking of anxiety symptoms (50\%), patient delays in seeking care (36\%), and lack of referrals to mental health providers (21\%).

Conclusion: The erroneous use of unspecified anxiety disorder diagnosis poses major barriers to receipt of appropriate evidence-based care for more specific psychiatric disorders that are amenable to evidence-based pharmacological and psychological treatment. The data from this study highlight areas for improvement in the diagnostic process to ensure accurate and timely anxiety diagnosis.

\section{Converging in Breast Cancer Diagnostic Screening: A Computational Model Proposal}

L. Garbayo ${ }^{1}$, W. Zadrozny ${ }^{2}$ and H. Hematialam ${ }^{3}$

${ }^{1}$ University of Central Florida

${ }^{2}$ University of North Carolina Charlotte

${ }^{3}$ University of North Carolina Charlotte, Computer Science Department

Background: Guidelines disagreement for breast cancer diagnosis screening generate undue clinical variability that may impact breast cancer early detection, leading eventually to overdiagnosis, overtreatment and overall loss of quality of life (Welch et al, 2016; Jorgensen et al, 2017; Zahl et al, 2019). It is known that the US Preventive task force (USPFSTF, 2009) effort to reduce such variability did not affect significantly previous screening patterns (Howard and Adams, 2012) and thus have not eliminated related peer disagreement. In this study we suggest that further convergence science strategies seems necessary. A new near-peer epistemic agency classification in reference to the medical sub-areas may be required, to better explain breast cancer disagreements in different fields such as oncology, gynecology, mastology, and family medicine.

Methods: Mixed methods. Computational, machine learning and formal modeling techniques, extended to topology; epistemology of expert disagreement.

Results: We generated a topological analysis of contradictions and disagreement of breast cancer screening guidelines with sheaves, while taking in consideration conceptual distance measures, to further explore in geometrical representation continuities and discontinuities in breast cancer screening diagnosis guidelines disagreements and contradictions (Zadrozny \& Garbayo, 2018). A technically rigorous visualization tool was produced.

Conclusion: New avenues to support clinical decision making for engaging in interdisciplinary medical experts to explore disagreement in diagnostic guidelines in breast cancer screening were generated. New studies are needed for implementation.

*Versions of stages of this work have been presented in computer science conferences and were accepted in logic and philosophy of science conferences. 


\title{
Promoting Appropriate Laboratory Test Utilization
}

\author{
B. Gunsolus ${ }^{1}$ and G. Singh ${ }^{2}$ \\ ${ }^{1}$ AU Health \\ ${ }^{2}$ Medical College of Georgia
}

Background: In the United States, it is estimated that $\$ 5$ billion, approximately $30 \%$ of all annual clinical laboratory testing, is attributed to inappropriate test utilization. In addition to the financial ramifications, inappropriate laboratory testing has been identified as a primary contributor to diagnostic error as well as imposing a significant workflow burden upon clinical laboratories performing the testing.

Inappropriate laboratory utilization encompasses four primary criteria: 1) a test is ordered when it is not necessary, 2) a test should be ordered, but it is not, 3) a test is ordered, but it is the wrong test, and 4) a test is ordered at the wrong time making test results inaccurate. Several approaches have been previously reported to promote appropriate test utilization. This project was undertaken to identify the types of inappropriate laboratory test utilization that are occurring and quantitate the extent of inappropriateness so that solutions for addressing the issue could be created.

Methods: A literature review was performed to identify tests other facilities reported inappropriate utilization. Test list included Protein C Activity, Factor V Level, Factor V Leiden mutation, Prothrombin G20210A mutation, Antinuclear Antibody (ANA), Lupus Anticoagulant, and Hemoglobin A1c (HgbA1c). Lists of 90 days of patient test results for each test except HgbA1c was generated from electronic health record (EHR) interfaced hc1.com software. Only 30 days of patient test results was generated for HgbA1c. Each test order was then reviewed in the EHR for appropriateness based on previously stated criteria.

Results: see table

Conclusion: Significant levels of inappropriate test utilization was identified on the seven tests reviewed. A multifaceted approach, including implementation of Diagnostic Management Teams, physician education, feedback on ordering practices, and electronic ordering solutions will be necessary to address these issues.

\begin{tabular}{lll}
\hline Test Name, $\boldsymbol{n}=$ total ordered & $n=\#(\%)$ Inappropriate & Reason Inappropriate \\
\hline Factor V Level, $\mathrm{n}=82$ & $\mathrm{n}=53(65 \%)$ & Wrong test selected, should be Factor V Leiden \\
Factor V Leiden, $\mathrm{n}=22$ & $\mathrm{n}=7(32 \%)$ & Duplicate genetic test \\
Prothrombin G20210A, $\mathrm{n}=44$ & $\mathrm{n}=14(32 \%)$ & Duplicate genetic test \\
HgbA1c, $\mathrm{n}=1909$ & $\mathrm{n}=439(23 \%)$ & Repeats $<60$ days from previous result \\
Protein C Activity, $\mathrm{n}=77$ & $\mathrm{n}=57(74 \%)$ & Tested while patient on anticoagulants \\
& Of those on anticoagulants & Decreased resulted due to anticoagulants, wrong \\
Lupus Anticoagulant, $\mathrm{n}=55$ & $\mathrm{n}=35(61 \%)$ & diagnosis of Protein C deficiency \\
& $\mathrm{n}=6(11 \%)$ & Wrong test selected, should be an ANA for SLE \\
$\begin{array}{l}\text { ANA ordered for elevated liver } \\
\text { functions, } \mathrm{n}=18\end{array}$ & $\mathrm{n}=44(80 \%)$ & Not necessary, ordered after normal PTT result \\
ANA results with titer $\geq 1: 160$ on & Had wrong additional confirmatory autoimmune \\
symptomatic patients, $\mathrm{n}=42$ & $\mathrm{n}=10(24 \%)$ & test (Sm Ab), correct is Smooth Muscle Antibody \\
\hline
\end{tabular}

\section{Predictors of Diagnostic Yield for CTA}

\author{
$\underline{\text { S. Harnois }}{ }^{1,2}$, S. Isaac ${ }^{1,2}$, H. Horstman ${ }^{1,2}$, J. Carwile ${ }^{1}$ and R. Trowbridge ${ }^{1,2}$ \\ ${ }^{1}$ Maine Medical Center \\ ${ }^{2}$ Tufts University School of Medicine
}

Background: The Wells score can be used to stratify patients by risk of pulmonary embolism (PE), with diagnostic imaging using computed tomographic pulmonary angiography (CTA) recommended for patients with high, but not low, pretest probability (PTP). Few studies have investigated risk factors for underutilization of objective risk stratification or overuse of CTA. We hypothesized that physicians overutilize CTA during times of high hospital occupancy or patient-provider ratios, resulting in a lower proportion of patients with a positive CTA finding (e.g., lower diagnostic yield). We conducted a retrospective analysis to examine the association between patient characteristics, hospital activity, and hospital operations with PTP and diagnostic yield.

Methods: We conducted a retrospective analysis of 1,019 patients aged =18 years who received a CTA at Maine Medical Center between January 1, 2016 and December 31, 2017. Patients identified as having chronic thromboembolic disease, small sub-segmental PE, or indeterminate scans were excluded. Trained physicians manually abstracted data on Wells score components; all other data was electronically abstracted from electronic medical records. We retrospectively calculated each patient's 2-tiered Wells score and categorized patients as PE likely (Wells $>4$ ) or unlikely (Wells =4) (intra-rater reliability, 0.72; inter-rater reliability, 0.47). We compared diagnostic yield and PTP across patient and hospital characteristics, and calculated statistical significance using Fisher's exact, Chi-square, and t-tests. 
Results: The retrospectively calculated 2-tiered Wells score had a sensitivity of $85.7 \%$, specificity of 32.6\%, PPV of $15.3 \%$, and NPV of $94.1 \%$. Overall diagnostic yield was $12.4 \%$. Diagnostic yield varied by sex (men, $15.3 \%$ vs. women, $10.2 \%$; $p=0.01$ ) and insurance payer (e.g., private, 18.5\%, Medicare, 10.7\%, Medicaid, 10.8\%; $\mathrm{p}=0.02$ ); however, PTP did not differ by sex or payer. Diagnostic yield did not differ by other demographic or operational characteristics, including day of week, time of day, or involvement of house staff or medical students. Associations between diagnostic yield, PTP, census, and complexity are currently underway.

Conclusion: We found a relatively lower diagnostic yield for CTA for women and patients with public insurance that was not explained by lower PTP. Future studies should continue to explore risk factors associated with low CTA diagnostic yield in order to characterize patient groups with low diagnostic yield and inform interventions to reduce unnecessary use of diagnostic imaging and associated exposure to ionizing radiation, expense, and delayed care.

\title{
Emotionally Evocative Patients in the Emergency Department: An Investigation of Providers' Reactions and Implications for Patient Safety
}

\author{
L. M. Isbell, J. Tager, K. Beals and G. Liu
}

University of Massachusetts Amherst

Background: It is well known that some patients evoke strong emotions in the Emergency Department (ED), a setting where risk for diagnostic error is high. Despite this, almost nothing is known about providers' experiences in response to emotionally-evocative patients, or effects on clinical reasoning and behavior. This research addresses this gap.

Methods: 94 ED providers (50 attending physicians, 44 registered nurses) wrote about three patient encounters: a happy/satisfying encounter, an angry/frustrating encounter, and a mental health encounter (a population known to evoke strong emotions). Participants rated their emotions and level of engagement in each encounter. A subset $(n=72)$ reported whether their emotions influenced their clinical reasoning and behavior in each encounter, the extent to which the influence was detrimental versus beneficial, and then described the influence.

Results: Providers' emotions varied depending on the patient encounter, $\mathrm{F}(14,348)=41.53, \mathrm{p}<.001$ (see Figure). Providers' emotions affected their clinical reasoning more frequently in angry encounters (62.5\%) than in mental health (47.2\%) and positive encounters (38.8\%), $2(2)=8.26, p=.016$. In angry encounters, physicians evaluated their emotions as more detrimental than beneficial $(p<.001)$; nurses evaluated them similarly $(\mathrm{p}=.60)$. In positive encounters, both physicians and nurses evaluated their emotions as more beneficial than detrimental (ps <.001), and as equally beneficial and detrimental in mental health encounters (ps $>.58$ ). Participants indicated that they provided the very best medical care in positive encounters (spending more time with patients, strongly advocating for patients, ordering additional tests). In angry encounters, providers described the adverse influence patients had on their mood, and described failing to provide the best medical care (making decisions based on what would get them out of the ED most quickly, acting less professionally and compassionately). Providers described a mix of positive and negative influences in the mental health encounter depending on their emotions.

Conclusion: ED providers experience a broad range of emotions and levels of engagement during patient encounters. Given that engagement is an important contributor to successful clinical reasoning, negative emotions can pose a significant risk to patient safety, whereas positive emotions may reduce this risk. Research is needed to identify interventions to mitigate this risk.

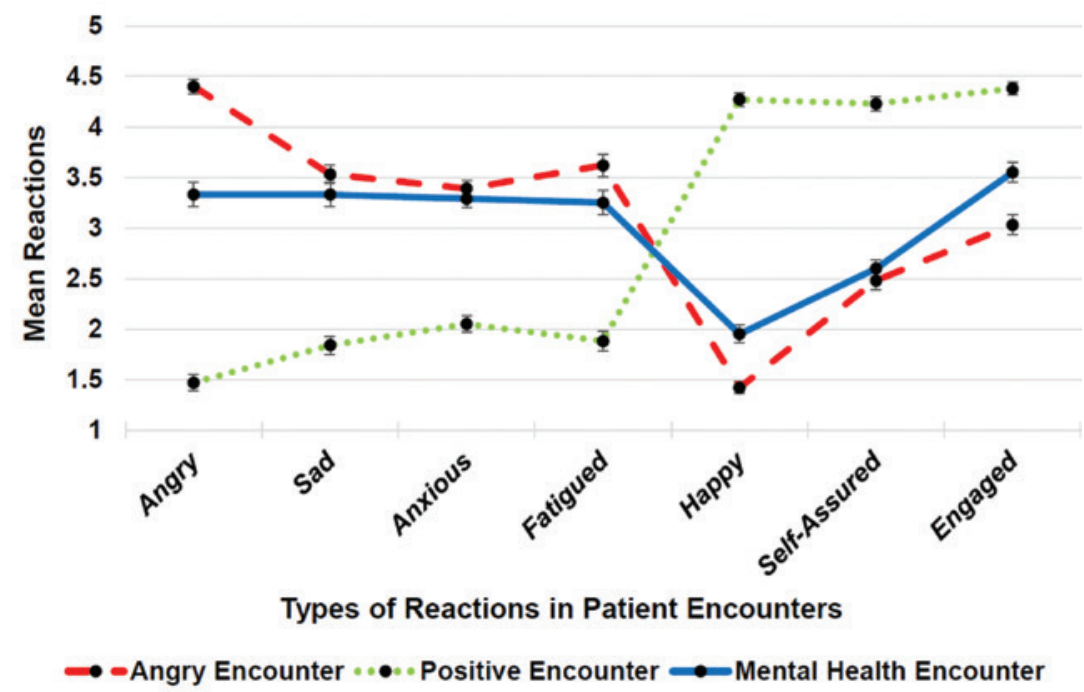

Sliding scales range from 1 (not at all) to 5 (very much). Error bars represent plus/minus 1 standard error. 


\title{
Early Diagnosis of Ruptured Abdominal Aortic Aneurysm: Initial Vital Signs in the Emergency Department
}

\author{
$\underline{\text { T. Kawahigashi }}{ }^{1}$ and K. Watanabe ${ }^{2}$ \\ ${ }^{1}$ Department of Emergency Medicine, Tokyo West Tokushukai Hospital \\ ${ }^{2}$ Department of Surgery, Tokyo West Tokushukai Hospital
}

Background: Ruptured abdominal aortic aneurysm (rAAA) is one of the most fatal surgical emergencies. Death is inevitable, unless timely repair is performed. The classical triad of abdominal and/or back pain, a pulsatile abdominal mass, and hypotension, is considered to be diagnostic. However, this triad may not manifest in its entirety. In particular, a previous report demonstrates that more than half of the patients were stable, and misdiagnosis was significantly more common in these patients than in those who were in shock. Here, we investigated the initial vital signs of patients with rAAA in the emergency department of our hospital.

Methods: In this retrospective analysis, we included rAAA patients who presented the Tokyo West Tokushukai Hospital between January 2014 and June 2019. Patients referred from other hospitals were excluded. Comparison between groups was performed using Fisher's exact test and the Mann-Whitney U test where appropriate.

Result: The medical records of 15 patients were available for review (14 men; mean age, 76.9 years). At presentation, 13 of the 15 patients $(86.7 \%)$ were hemodynamically stable (systolic blood pressure $=90 \mathrm{mmHg}$ ). Overall 30 -day mortality was $40 \%$ and was higher in patients in shock at presentation than in those who were hemodynamically stable $(100 \%$ vs. $30.8 \%, p=0.14)$. None of the patients showed any other signs of shock such as tachycardia or tachypnea. Mean time from presentation to diagnosis was longer in patients without hemodynamic shock (1.9hr vs 1.0hr, $\mathrm{p}=0.29)$. Median time from onset to presentation at the hospital was significantly longer for patients without shock (8.0hr vs 4.0hr, p=0.93).

Conclusion: Several rAAA patients may present without hemodynamic shock or any other signs of shock, which may lead to diagnostic delays. Moreover, patients without hemodynamic shock tend to have a longer time span between onset and present; this may also contribute to diagnostic delay because most physicians expect rAAA patients to present with "sudden" onset of pain. Shock was associated with poor outcomes; therefore, prompt diagnosis and treatment before the development of shock is important. While these differences were not statistically significant, a larger study might have revealed a statistical difference. Physicians need to be extremely vigilant of this condition while diagnosing patients with abdominal, back, or chest pain, even if they do not complain of sudden onset pain and are hemodynamically stable.

\section{High Rates of Misdiagnosis Characterize Patients Referred for Evaluation of Lyme Disease}

\author{
$\underline{\text { T. Kobayashi }}^{1,2}$, Y. Higgins ${ }^{2}$, G. Yenokyan ${ }^{3}$, M. T. Melia ${ }^{4}$ and P. G. Auwaerter ${ }^{2,4}$ \\ ${ }^{1}$ Infectious Disease, University of Iowa Hospitals and Clinics, Iowa City, IA \\ ${ }^{2}$ Sherrilyn and Ken Fisher Center for Environmental Infectious Diseases, Johns Hopkins University School of Medicine, Baltimore, MD \\ ${ }_{3}^{3}$ Johns Hopkins Biostatistics Center, Johns Hopkins Bloomberg School of Public Health, Baltimore, MD \\ ${ }^{4}$ Infectious Disease, Johns Hopkins University School of Medicine, Baltimore, MD
}

Background: While Lyme disease is the most common vector-borne infection in the U.S., studies have demonstrated that Lyme disease is over-diagnosed in some populations. However, little is known about the diagnoses among these misdiagnosed patients who were referred for Lyme disease but had no supporting evidence of the infection.

Methods: A retrospective observational cohort study of patients referred to infectious disease clinic of the Johns Hopkins University of School of Medicine between 2000-2013, with a presumed diagnosis or patient concern for Lyme disease, was performed to investigate diagnoses of their presenting complaints. Persons younger than 12 years old were excluded. Diagnoses of recent or past Lyme disease used standard CDC criteria. Patients without recent Lyme disease were further reviewed for diagnoses of their presenting health problems, including all electronic medical records within the health system.

Results: Of 1261 referred patients, only 200 (15.9\%) patients were diagnosed with acute or recent Lyme disease, and 1061 (84.1\%) patients did not have evidence suggesting Lyme disease as a cause of their presenting symptoms, yet most received antibiotic. The mean age of those 1061 patients was 45.6 years old, and 685 (64.6\%) patients were female. Of the 1061 patients without Lyme disease, the top seven diagnoses reached were: no diagnosis, usually due to lack of follow-up testing or further evaluation in 364 (34.3\%) patients, anxiety/depression in 222 (20.9\%) patients, fibromyalgia in 120 (11.3\%) patients, chronic fatigue syndrome in 77 (7.3\%) patients, migraine disorder in 74 (7.0\%) patients, osteoarthritis in $62(5.8 \%)$ patients and sleep disorder/apnea in 47 (4.4\%) patients. Other serious health conditions misattributed to Lyme disease included multiple sclerosis (15 patients), Parkinson's disease (12), thyroid disease (11), alcohol abuse (10), inflammatory bowel disease (8), amyotrophic lateral sclerosis (7), rheumatoid arthritis (5), sarcoidosis (5), substance abuse (4), systemic lupus erythematosus (2), pregnancy (1), vasculitis (1), amyloidosis (1), myasthenia gravis (1), metastatic lung cancer (1), metastatic prostate cancer (1) and hepatitis C (1).

Conclusion: The majority of patients referred for Lyme disease did not have any evidence of this infection. Most were misdiagnosed, suggesting that either alternative medical practices or misapplication of Lyme disease testing may place patients at risk for delay in receiving proper diagnosis and treatment. 


\section{Documentation of Clinical Reasoning in Admission Notes of Hospitalists: Validation of the CRANAPL Assessment Rubric}

$\underline{\text { S. Kotwal }}^{1}$, D. Klimpl' ${ }^{2}$ S. Tackett ${ }^{3,4}$, R. Kauffman ${ }^{1}$ and S. Wright ${ }^{3}$

${ }^{1}$ Department of Medicine, Division of Hospital Medicine, Johns Hopkins Bayview Medical Center, Johns Hopkins University School of Medicine, Baltimore, MD

${ }^{2}$ Division of Hospital Medicine, University of Colorado, Denver, Colorado

${ }^{3}$ Department of Medicine, Division of General Internal Medicine, Johns Hopkins Bayview Medical Center, Johns Hopkins University School of Medicine, Baltimore, MD

${ }^{4}$ Biostatistics, Epidemiology And Data Management (BEAD) Core, Johns Hopkins School of Medicine, Baltimore, MD

Background: Thoughtful and high-quality clinical documentation transmits one's clinical reasoning, is essential for patient safety and professional responsibility. There are no accepted standards for assessing documentation of clinical reasoning. We undertook this study to establish a metric to evaluate hospitalists' documentation of clinical reasoning in assessment and plan (A\&Ps) section of admission notes.

Methods: This was a retrospective study reviewing admission notes of hospitalists at three hospitals between January 2014 and October 2017. Admission notes were included for patients hospitalized with a diagnosis of either fever, syncope/dizziness, or abdominal pain. 650 admission notes were randomly identified. Notes were excluded if they were not on the hospitalists' service, or if a specific diagnosis had been confirmed in the emergency department. No more than 3 notes written by any single provider was analyzed. We developed the 'Clinical Reasoning in Admission Notes Assessment \& Plan' (CRANAPL) tool to assess the comprehensiveness of clinical reasoning documented in the A\&Ps of admission notes. Two authors scored all A\&Ps using the finalized version of the CRANAPL tool and assessed each A\&P using 2 single-item broad ratings: a 'global clinical reasoning' and a 'global readability/clarity' measure. Data were deidentified prior to scoring. Validity evidence for the tool was established based on Messick's unified validity framework: content, response process, internal structure, and relations to other variables.

Results: 120 hospitalists wrote the 285 admission notes. The figure shows the tool and distribution of scores for each rater, the mean score for both raters for the total CRANAPL score, and the interclass correlation measuring inter-rater reliability for the total CRANAPL score. Associations between CRANAPL scores and both the global clinical reasoning and global score for note readability/clarity were positive and statistically significant (both $\mathrm{p}<0.001$ ). Notes from academic hospitalists had higher CRANAPL scores [7.4 (SD 2.0) \& 6.6 (SD 2.1)] than did those written by hospitalists working at community hospital [5.2 (SD 1.9)], $\mathrm{p}<0.001$. In multivariate regressions after adjusting for covariates, higher scores on the CRANAPL tool were seen for notes from American Medical Graduates as compared to International Medical Graduates, and males as compared to females (both $\mathrm{p}<0.05$ ).

Conclusions: This study represents a first step to characterize clinical reasoning documentation in hospital medicine. With validity evidence established for CRANAPL, it may be possible to assess the documentation of clinical reasoning. This may be useful for improving the diagnostic process among hospitalists and for reducing medical errors. 


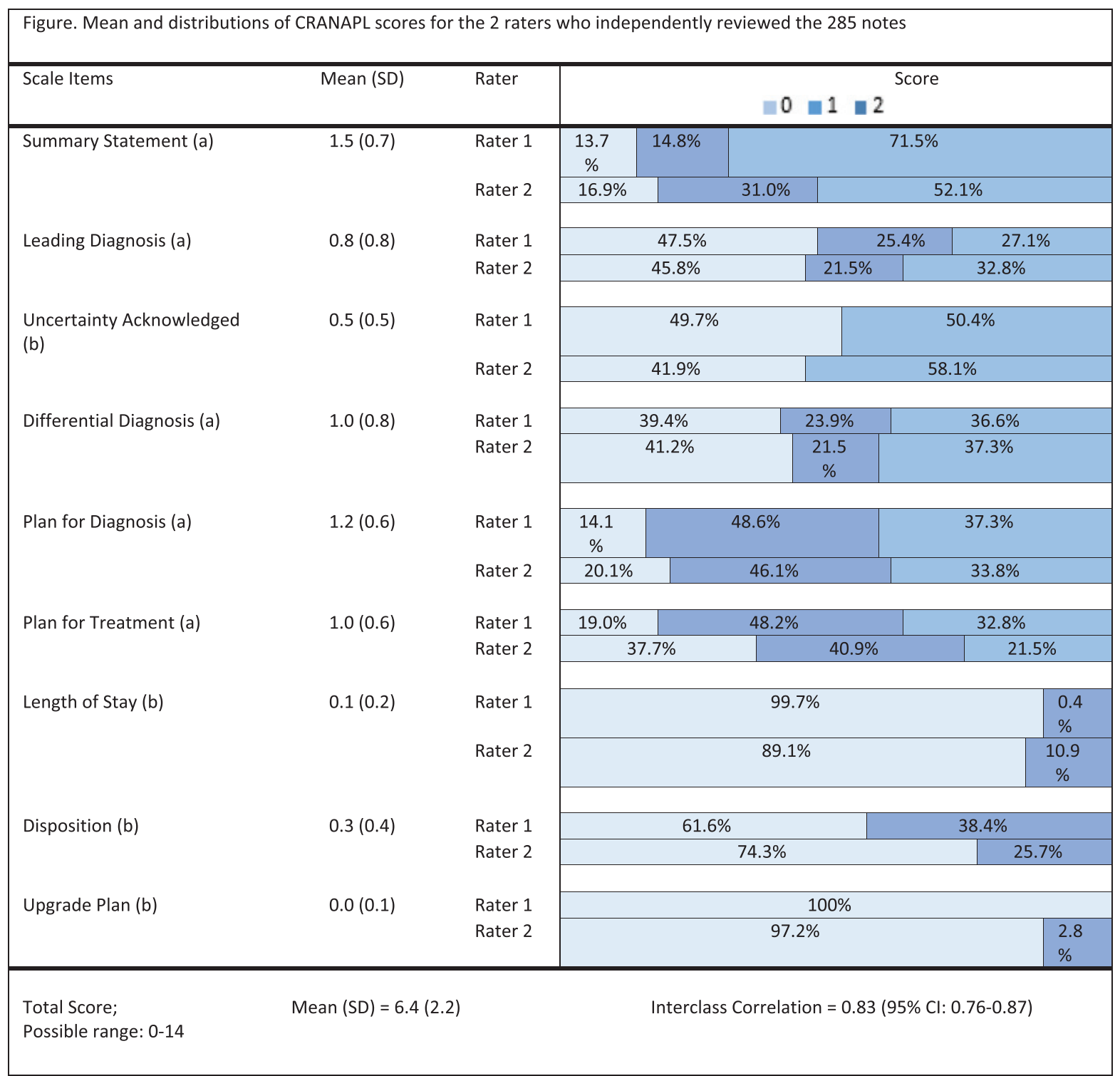

[a] - Item scored 0 to 2.0 = absent, $1=$ present but no explanation, $2=$ present and explanation noted [b] - Item scored 0 or $1.0=$ absent, 1 = present

\section{Teaching Reflective Reasoning through Explaining on Video}

J. Kuhn ${ }^{1,2}$, S. Mamede'2, P. van den Berg'1, L. Zwaan², P. Bindels ${ }^{1}$ and T. van Gog ${ }^{3}$ ${ }^{1}$ General Practice, Erasmus MC, Rotterdam, Netherlands, 2iMERR, Institute of Medical Education Research Rotterdam, Erasmus MC, Rotterdam, Netherlands, ${ }^{3}$ Pedagogical and Educational Sciences, University Utrecht, Utrecht, Netherlands

Background: The method of structured reflection (Mamede, Schmidt et al. 2008) was developed as a means to enhance physicians' critical thinking, which is a crucial part of medical diagnostic. It has been shown that structured reflection can improve diagnostic accuracy on complex cases and can remedy diagnostic errors caused by cognitive bias. An open question, however, is whether the procedure can also be learned and then autonomously applied when solving future cases without instructions how to reason. A recent study found (Hoogerheide, Renkl et al. 2019), that students effectively acquired problem-solving skills in physics, when they first studied an example showing how to solve a problem, and then explained the learned content on video to a fictitious peer. 
We investigated whether engaging in video explaining is also effective for teaching the procedure of structured reflection to general-practice residents so that could apply it autonomously on future cases.

Methods: Fifty-six residents in general practice were randomly assigned to one of two conditions in a learning session. In the control condition, participants diagnosed six cases without further instructions. Participants in the explanation condition first studied three examples of cases solved with structured reflection. After this, they made two web-cam recordings in which they explain to a fictitious peer (1) the steps of structured reflection, and (2) how to apply it on a given case. On a delayed test (one or two weeks later), all participants diagnosed a new set of four cases while thinking aloud. We measured whether the key elements of structured reflection were present in the think-aloud protocols and the diagnostic accuracy.

Results: The number of structured reflection elements did not differ between the conditions, $\mathrm{F}(1,43)=.37$, $\mathrm{p}=.544$, ?p2 = .01, neither did diagnostic accuracy, $\mathrm{F}(1,54)=.61, \mathrm{p}=.440, ? \mathrm{p} 2=.01$. Note that only 45 residents were included in the first analysis because for 11 residents the recordings of the think-aloud task were not recorded correctly.

Conclusion: Against expectations, we did not find that practicing with structured reflection led to more reflective reasoning or improved diagnostic accuracy when diagnosing future cases. One explanation is that residents are too experienced to change their habit of diagnosing cases in only one session. The intervention might be more effective for fostering critical thinking in medical students.

\title{
How Much do Clinical Decision Support Systems Improve Care and Diagnosis? A Meta-analysis of Controlled Clinical Trials
}

\author{
J. L. Kwan ${ }^{1,2}$, L. Lo ${ }^{3}$, J. Ferguson ${ }^{4}$, H. Goldberg ${ }^{5}$, J. P. Diaz Martinez ${ }^{6}$, J. M. Grimshaw ${ }^{7,8}$ and K. G. Shojania ${ }^{2,3,9}$ \\ ${ }^{1}$ Department of Medicine, Mount Sinai Hospital, Toronto, Ontario, Canada \\ ${ }^{2}$ Department of Medicine, University of Toronto, Toronto, Ontario, Canada \\ ${ }^{3}$ Centre for Quality Improvement and Patient Safety, University of Toronto, Toronto, Ontario, Canada \\ ${ }^{4}$ Western University, London, Ontario, Canada \\ ${ }^{5}$ Faculty of Medicine, University of Toronto, Toronto, Ontario, Canada \\ ${ }^{6}$ Biostatistical Research Unit, Toronto General Hospital, Toronto, Ontario, Canada \\ ${ }^{7}$ Clinical Epidemiology Program, Ottawa Hospital Research Institute, Ottawa, Ontario, Canada \\ ${ }^{8}$ Faculty of Medicine, University of Ottawa, Ottawa, Ontario, Canada \\ ${ }^{9}$ Department of Medicine, Sunnybrook Health Sciences Centre, Toronto, Ontario, Canada
}

Background: Improving care - including diagnosis - through clinical decision support systems (CDSSs) at the point of care represents one of the main incentives for implementing electronic health records. Most systematic reviews have identified predictors of positive effects without reporting the typical magnitude of improvement. We sought to: 1) quantify the typical improvement in processes of care conferred through the use of CDSSs delivered at the point of care; and, 2) identify any study or CDSS features consistently associated with larger effects.

Methods: MEDLINE from inception until August 15, 2018 was searched for randomized or quasi-randomized clinical trials of CDSSs delivered to physicians at the point of care. Two authors independently screened studies for eligibility, extracted data, and evaluated for risk of bias, with disagreements resolved in discussion with a third author. Hierarchical multilevel meta-analysis was performed using random effects models with adjustment for clustering. Univariate and stepwise multivariate meta-regression analyses explored treatment associations with different subgroups. Our primary outcome was adherence to target processes of care (e.g., the percentage of patients who received the care recommended by the CDSS). To standardize the direction of effects, we reverse coded results for interventions aimed at decreasing target processes of care.

Results: Across 106 studies (92 randomized trials and 14 quasi-randomized trials) reporting 120 comparisons, a median of 38.7\% (IQR 16.4\%$61.3 \%$ ) of patients in control groups received the desired process of care. For patients in the intervention groups, CDSSs achieved an absolute improvement in adherence to desired care of 6.2\% (95\% CI: 4.3\%-8.2\%). In univariate meta-regression analyses, adherence was incrementally improved by $6.5 \%$ (95\% CI: 1.1\%-22.2\%, p < 0.05) for those CDSSs requiring acknowledgement plus documentation of a reason for the action taken, and by $15.2 \%$ (95\% CI: 8.2\%-22.2\%, p < 0.001) for interventions conducted in pediatric settings compared to those that did not. Stepwise meta-regression analyses additionally showed that CDSSs using the EPIC platform achieved absolute improvements of 4.4\% (95\% CI: $0.2 \%$ $8.6 \%, p=0.04$ ) compared to those that did not. Seven trials evaluated the effects of CDSSs on diagnosis either directly or indirectly; of these, only 3 were found to have substantially larger effects (9.5\%-19\%).

Conclusion: Although a minority studies report substantial improvements in care, most CDSS interventions achieve fairly small improvements across a range of processes. Few studies specifically evaluated diagnosis. Future research must identify design features for CDSSs - including those pertaining to diagnosis - that reliably confer larger improvements in care. 


\title{
Subtypes of Neurovascular Disease after Emergency Department Headache Visit
}

\author{
A. L. Liberman ${ }^{1}$, J. Lu², K. Moncrieffe ${ }^{1}$, N. Cheng ${ }^{1}$, D. L. Labovitz ${ }^{1}$ and R. B. Lipton ${ }^{1}$ \\ ${ }^{1}$ Department of Neurology, Montefiore Medical Center, Albert Einstein College of Medicine, Bronx, NY \\ ${ }^{2}$ Albert Einstein College of Medicine, Bronx, NY.
}

Background: Headache is a common presenting symptom of dangerous neurovascular disease. A recent multi-state administrative claims study reported that $0.5 \%$ of headache patients discharged from an Emergency Department (ED) visit had a probable ED misdiagnosis of dangerous neurological disease including stroke. However, detailed characterization of the type of neurovascular disease hospitalizations following ED headache visits, which may inform strategies for stroke detection and prevention among headache patients, has not been described.

Methods: We conducted a retrospective study at a single academic medical center, Montefiore Medical Center (MMC), from 9/1/2013 to 9/1/2018. We queried administrative claims data from the four MMC EDs located in Bronx, New York. All adult patients with an ED visit resulting in a direct discharge home (ED treat-and-release visit) diagnosed with a benign headache disorder in the ED were included in our cohort. Benign headache diagnoses were identified using ICD-9-CM and ICD-10-CM codes. The first subsequent hospitalization for cerebrovascular disease within 365 days of index ED visit at an MMC facility was identified using ICD-9-CM and ICD-10-CM codes. Medical records and imaging was then reviewed to confirm the presence of neurovascular disease and to classify disease subtype using a previously validated schema. The rate of hospitalization for confirmed neurovascular disease within 365 days of ED visit is reported as a measure of possible ED missed diagnosis. Results: We identified a total of 28,121 patients with an ED treat-and-release visit for benign headache. The mean age of the cohort was 43.1 (SD: 16.7 ) and 71.8\% were women. After detailed medical record and imaging review, 114 patients had a confirmed hospitalization for neurovascular disease ( $0.4 \%$ of the entire cohort). Neurovascular hospitalizations occurred at a median of 118 days (IQR: 51-215) after index ED visit. Among those with neurovascular disease, ischemic strokes $(76.3 \% ; \mathrm{n}=87)$ were most common; intracerebral hemorrhages $(7.9 \%)$, subdural hematomas (4.4\%), transient ischemic attacks (4.4\%), subarachnoid hemorrhages (3.5\%), cerebral vein thromboses (1.8\%), and other ischemia (1.8\%) also occurred. Ischemic stroke mechanistic subtypes included: cryptogenic (32.2\%); lacunar (25.3\%), cardioembolic (13.8\%), large vessel (11.5\%), other determined cause (13.8\%), and conflicting mechanism (3.4\%); no patients had migrainous infarctions.

Conclusion: Among patients with a treat-and-release ED visit for benign headache, ischemic stroke was the most common reason for subsequent confirmed neurovascular hospitalization. Improved detection of cerebral ischemia in the ED as well as additional tools to improve risk-stratification among headache patients are warranted.

\section{Factors Associated with Potentially Missed Diagnosis of Appendicitis in the Emergency Department in a Private Insurance Claims Database}

\author{
P. Mahajan ${ }^{1}$, T. Basu' ${ }^{2}$ C. W. Pai ${ }^{1}$, H. Singh ${ }^{3}$, N. Peterson ${ }^{3}$, M. F. Bellolio ${ }^{4}$, S. K. Gadepalli5 and N. S. Kamdar ${ }^{2}$ \\ ${ }^{1}$ Department of Emergency Medicine, University of Michigan \\ ${ }^{2}$ Institute for Healthcare Policy and Innovation, University of Michigan \\ ${ }^{3}$ Houston Veterans Affairs Center for Innovations in Quality, Effectiveness and Safety \\ ${ }^{4}$ Departments of Emergency Medicine and Health Sciences Research, Mayo Clinic \\ ${ }^{5}$ Division of Pediatric Surgery, University of Michigan
}

Background: Appendicitis, a common diagnosis with abdominal pain and other vague symptoms, may be missed upon initial emergency department (ED) presentation. We sought to compare patients with appendicitis-associated symptoms diagnosed as appendicitis at the initial ED visit (same day diagnosis, SDD) with those diagnosed within 30 days after the initial ED visit (potentially missed appendicitis, PMA).

Methods: We performed a retrospective analysis of de-identified claims data (2010-2017) of commercially insured patients using the Clinformatics ${ }^{\circledR}$ DataMart (OptumInsight, Eden Prairie, MN) based on the “Symptom-Disease Pair Analysis of Diagnostic Error” look-back approach. We included all adults ( $=18$ years) and children ( $<18$ years) with a diagnosis of appendicitis and evaluated those with a prior ED visits within 30 days with any of the five undifferentiated symptoms, abdominal pain, constipation, vomiting/nausea, fever, and diarrhea. We reported adjusted odds ratios (AORs) of PMA for abdominal pain and its combinations with other symptoms and AORs from models stratified by each of the five appendicitis-associated symptoms. Variables included in the multivariable logistic regression models for each of the five symptom cohorts were demographics, Elixhauser comorbidity index, and abdominal diagnostic imaging (computed tomography [CT], ultrasonography [US], X-ray).

Results: Among 123,711 patients with appendicitis diagnosis and prior ED visits with related symptoms, the PMA rate was 6.0\% for adults and $4.4 \%$ for children. Patients who presented with abdominal pain only (adult AOR 0.65 [95\% CI 0.62-0.69], P < .001; children AOR 0.79 [0.69-0.90], $\mathrm{P}<.001$ ) or in combination with vomiting/nausea (adult AOR 0.90 [0.84-0.97], P = .003; children AOR 0.84 [0.71-0.98], P = .03) were less likely to have PMA. Stratified by presence of undifferentiated symptom, females (adult AOR range 1.19-1.68, children AOR range 1.551.80), comorbidity index 2 or higher (adult AOR range 3.33-5.00; children AOR range 2.17-4.12) were more likely to be potentially missed. Adult patients who received CT scans at the initial ED visit were less likely to be missed (AOR range 0.41-0.63). On the other hand, adults only with an abdominal X-ray in the ED were more likely to be missed (adult AOR range 2.73-4.07). 
Conclusion: We reported estimates of potentially missed appendicitis using claims data from a large dataset of commercially insured patients. Female sex and higher comorbidity index were factors associated with higher risk of missed appendicitis regardless of age. Adults who received CT scans were more likely to be diagnosed at index ED presentation.

\title{
Sepsis Uncertainty: Translating Theoretical Models into Practical Understanding of Time-to-Antibiotics Among Patients with Suspected Sepsis and High Severity of Illness
}

\author{
K. E. Raffel, P. A. Prasad, A. M. Esmaili, K. D. Liu and K. N. Kangelaris \\ University of California, San Francisco
}

Background: The 2016 Surviving Sepsis Campaign guidelines recommend antibiotic administration within 1-hour of presentation ('timezero') for patients with sepsis. While early antibiotics are associated with reduced mortality in the sickest patients, this policy has been criticized for its one-size-fits-all approach and risk for over-diagnosis.

Recent models have called for antibiotic timing that mirrors clinician assessment of illness severity-- risk of death from acute physiologic derangements and/or medical complexity. We translate acute physiologic derangement into practical clinical measures and utilize these to stratify time to antibiotic performance by severity of illness among patients with suspected sepsis.

Methods: We extracted data from the electronic health record (EHR) on all adults presenting to a single academic ED between 6/1/2012 and 12/31/2016 with suspected sepsis (blood cultures and dose of antibiotics in first 72 hours of presentation) and stratified them by presence/ absence of confirmed sepsis (change from baseline Sequential Organ Failure Assessment Score (SOFA) =2 by 48 hours and minimum 4 antibiotic days unless death/discharge to hospice sooner or validated sepsis discharge code). Time-stamped EHR-derived measurement of time to major organ dysfunction (lactate $=4$, Glasgow Coma Scale $(\mathrm{GCS})=12$, shock requiring vasopressor or 02 saturation $/ \mathrm{FiO} 2=300$ ) and time to first IV antibiotic from either ED presentation or major organ dysfunction were collected.

Results: 16,612 patients presented to the ED with suspected sepsis within 72 hours of ED presentation; 9,674 (58\%) met sepsis criteria within 48 hours of ED presentation. Of all suspected sepsis, 6,544 patients (39\%) had major organ dysfunction-- $47 \%$ of sepsis and $28 \%$ non-sepsis groups $(\mathrm{P}<0.001)$. Median time from presentation to major organ dysfunction was similar in the sepsis and non-sepsis groups $(1.4 \mathrm{v}$ 1.6 hours, $\mathrm{P}=0.24)$. For these patients, median time from presentation to antibiotics was 2.3 versus 3.3 hours in sepsis and non-sepsis groups $(\mathrm{P}<0.0001)$; median time from major organ dysfunction to antibiotics was 0.53 versus 1.13 hours $(\mathrm{P}<0.0001)$.

Conclusion: The median time from ED presentation to antibiotic receipt for patients with severe organ dysfunction, regardless of ultimate sepsis/non-sepsis status, was 2-3 hours, outside of current guidelines. However, median time from major organ dysfunction to antibiotics was $=1$ hour. This highlights the need to re-evaluate the 'time-zero' of time to antibiotics to both ensure this quality measure reflects clinical processes but, more importantly, to better understand what time point matters for patient outcomes- ED presentation-antibiotic time versus major organ dysfunction-antibiotics.

\section{EHR Design to Aid Clinical Cognition}

\author{
$\underline{\text { Y. Senathirajah }}^{1}$, E. Borycki², A. Kushniruk ${ }^{2}$ and K. Cato ${ }^{3}$ \\ ${ }^{1}$ University of Pittsburgh, Pittsburgh, PA \\ ${ }^{2}$ University of Victoria, Victoria, BC, Canada \\ ${ }^{3}$ Columbia University School of Nursing, New York, NY
}

Background: Information presentation in electronic health records (EHRs) can have profound effects on information apprehension and clinical reasoning. Display fragmentation, i.e. the placement of related and necessary information in different parts of the record, requires extensive navigation, imposing extrinsic cognitive load, burdening working memory, and affecting diagnostic performance. Other problematic features of current EHRs include inability to mark up or otherwise distinguish more salient data, excessive alerting, and the need to exert hand-eye coordination in suboptimal navigation designs. These are blamed for user dissatisfaction leading to physician burnout, as well as increasing the difficulty of diagnosis, incomplete information gathering due to time constraints, leading to premature satisficing, and the possibility of errors in both diagnosis and treatment ordering.

Methods: we utilized analysis of EHR navigation structures, usage logfiles, eyetracking studies of users conducting their normal EHR activities, interviews, and thinkaloud protocols to understand clinical reasoning in current EHRs and in experimental interaction designs including a user-composable test EHR and visualizations. 20 clinicians complex patient cases, were compared using a composable EHR and the usual commercial EHR interfaces which have fragmented displays. An online study of clinicians using composable interfaces to diagnose patient cases and transmit information to colleagues was analyzed for the extent of distortions and effect on users' ability to catch deliberate errors or omissions. Simulation studies in which the composable interface is used in complex multi-patient emergency department scenarios can help us understand the effect of EHR design on ability to handle interruptions.

Results: Overly complex navigation structures can require dozens of clicks required for typical tasks. Eyetracking studies and thinkaloud protocols reveal a six-fold reduction in time with the composable approach, and evidence for cognitive load reduction and increased user 
satisfaction. Users cite juxtaposition of information in the composable approaches and compact visualization as an aid to clinical reasoning. Configurable marking which raises salient data to the surface can affect users' initial apprehension of the data and clinical reasoning pathways, avoiding anchoring.

Conclusion: Composable EHR approaches can reduce extrinsic cognitive load. New technological approaches such as voice control, visualization techniques, and gaming to adjust clinician heuristics suggest new lines of research to support clinical reasoning.

\title{
Performance of an e-Trigger to Detect Missed Stroke Diagnosis in Patients with Headache or Dizziness Symptoms in Emergency Department
}

\author{
V. Vaghani ${ }^{1}$, L. Wei ${ }^{1}$, U. Mushtaq1, A. Zimolzak ${ }^{1}$, D. F. Sittig ${ }^{2}$ and H. Singh ${ }^{1}$ \\ ${ }^{1}$ Michael E. DeBakey Veterans Affairs Medical Center and Baylor College of Medicine \\ ${ }^{2}$ School of Biomedical Informatics at University of Texas Health Science Center
}

Background: Statistical analysis of large data sets using the 'Symptom-Disease Pair Analysis of Diagnostic Error' (SPADE) approach has been proposed as a method to determine frequency of misdiagnosis-related harm. Conversely, electronic triggers (or e-trigger tools) first mine vast amounts of clinical and administrative data and are followed by validation record reviews on highly-selected records to confirm presence/ absence of diagnostic error. We developed an e-trigger of two symptom-disease pairs (headache and dizziness followed by stroke) and examined its performance for identifying missed opportunities in stroke diagnosis in emergency departments (ED).

Methods: We developed the stroke e-trigger using a knowledge discovery framework, the Safer Dx Trigger Tools Framework. Using a "lookback" approach, we conducted record reviews for all patients admitted to VA facilities in 2016-17 for stroke (based on ICD-10 codes) that also had a "treat-and-release" ED visit with discharge diagnosis as headache or dizziness within prior 30 days. A trained physician reviewed each record for a) presence/absence of red-flags and stroke risk factors associated with stroke or transient ischemic attack (based on American Stroke Association guidelines, such as speech abnormalities, limb weakness, hypertension, hyperlipidemia); and b) actions in response to red-flags, such as neurological consultation or appropriate imaging. We defined missed opportunity in diagnosis (MODs) when no additional action or evaluation was undertaken despite stroke-related red-flags and potential missed opportunity in diagnosis (P-MODs) when red-flags at ED visit were absent but patient had multiple stroke risk factors and abnormal or incomplete neurological examination. All MODs and PMODs and a random sample of non-missed cases were discussed in team settings with two additional physicians.

Results: The stroke e-trigger was applied to an electronic health record datawarehouse containing $>9$ million patient-records and yielded 395 trigger positive records. Of 100 trigger positive cases reviewed thus far, 25 cases had MODs or P-MODs, 46 were miscoded as having stroke, 28 were not missed, and 1 was inconclusive. Thus, the PPV of stroke e-trigger thus far is $25 \%$. Average numbers of red flags in missed vs. nonmissed groups was comparable ( 0.32 in missed vs 0.18 in non-missed; $p=0.44$ ) and so were risk-factors ( 3.16 in missed vs 3.11 in non-missed; $\mathrm{p}=0.89$ ).

Conclusion: A symptom-disease pair based stroke e-trigger using data mining and confirmatory record reviews identifies patients with missed strokes with a modest PPV. Upon e-trigger validation of the SPADE approach, we found that several patients did not encounter delays in stroke diagnosis or had miscoded strokes.

\section{Diagnostic Reboot: Food for Thought}

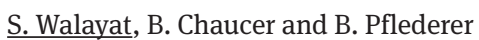

University of Illinois, Peoria, Department of Internal Medicine

Background: Diagnostic errors contribute to the morbidity and mortality of patients. They are the leading cause of medical malpractice claims and contribute to 6-17\% of hospital adverse events (1). It has been proposed that methods that encourage slow analytical reasoning may improve diagnostic accuracy. Real world data however is lacking. In this pilot study, we created and utilized a novel diagnostic tool (Diagnostic Reboot) and assessed its practical efficacy in the inpatient setting on improving diagnostic outcomes.

Methods: This was a prospective pilot study which involved 2 University Hospitalist Adult Teaching Service (UHATS) teams. Senior Residents were instructed to use the Diagnostic Reboot (DxR) tool whenever a patient aged 19-99 was identified who had an uncertain diagnosis after 24 hours of hospital admission. The duration of the pilot study was one month. Data sheets after completion from residents were stored in a locker, with no direct patient identifiers used. The study was approved by IRB.

Result: Eight patients admitted to UHATS were identified as complex a senior resident on two UHATS teams over a 1 month period. Senior A took 15 minutes on average for the DxR exercise while senior B took an average of 35 minutes. Leading diagnosis was excluded in 2 out of 8 patients (25\%), new leading diagnosis was made in 6 out of 8 patients (75\%). New diagnostic plan was made in $100 \%$ of patients who underwent the diagnostic reboot. A new consultation was requested in 3 out of 8 (37.5\%) of patients. The participating residents were also surveyed after the study to assess the practical value of the DxR. In spite of the differences in time spent with the DxR, both concluded that the tool was practical for use in their usual workflow on the inpatient service. Both residents felt it was helpful in reaching a clearer diagnosis.

Conclusion: This study demonstrated that the use of the Dx Reboot resulted in subjectively improved diagnostic reasoning amongst residents. The data suggest it was helpful in reaching a new leading diagnosis in $75 \%$ of patients and helped make a new diagnostic plan in $100 \%$ 
of patients. Our results suggest that the use of Dx Reboot may increase diagnostic accuracy while improving diagnostic reasoning of residents. Further studies with greater power are needed to validate these initial findings.

\section{References:}

1. Ball J, Balogh E, Miller BT, editors. Improving diagnosis in health care. National Academies Press; 2015.

\section{Analysis of Diagnostic Errors and Cognitive Biases in Japan Based on Physician’s Self-reflection}

\section{T. Watari}

Shimane University Hospital, Postgraduate clinical training center

Background: Previous research in the US has shown that diagnostic errors are a major cause of medical lawsuits. However, little is known about how much a diagnostic error contributes to medical malpractice claims in Japan. Therefore, we conducted a retrospective study on the background and effects of diagnostic errors using the largest internet database of claims in Japan.

Methods: The largest malpractice claims database in Japan (Westlaw Japan K.K) was used to identify diagnostic error-related and non-related malpractice claims. We excluded the duplicated and non-medical claims including intentional crimes, robbery, money troubles, and veterinary claims, using the exclusion criteria. We collected the following data: age and sex of the patient, specialty of the doctor, place or clinical setting, hospital size, initial and final diagnosis, acceptance amount, death, presence of sequelae, etc. All amounts were adjusted with the consumer price index.

Results: In the 1972 medical malpractice claims analyzed, the median age of patients was 33.5 years (IQR: 10-54), and 53.7\% were male. The medical outcomes included deaths in 939 cases (53.3\%). In total, 725 of 1972 malpractice claims (37.4\%) were diagnostic error-related cases. On comparing the diagnostic error-related $(n=709)$ and non-related $(n=1093)$ cases, the mortality rates were found to be $62.3 \%$ and $45.5 \%$, respectively ( $\mathrm{p}$-value $<0.001$ ); cases of internal medicine were significantly higher in the former $30.5 \%$ and $19.0 \%$, respectively (p-value $<0.001$ ); small-size hospitals were higher in the former $23.3 \%$ and $17.3 \%$, respectively (p-value $=0.002$ ); cases in general outpatient and emergency room were higher in the former $42.9 \%$ and $15.8 \%$, respectively (p-value $<0.001$ ); cases during the night shift $17.5 \%$ was higher than case of day shift $12.0 \%$ (p-value $<0.001$ ). Additionally, the acceptance rates of diagnostic error-related and non-related claims were $63.6 \%$ and $46.0 \%$, respectively ( $p$-value $<0.001$ ), and the median claim payments of both groups were $\$ 231181$ (IQR $\$ 50150-\$ 484546$ ) and $\$ 136,363$ (IQR \$30554-\$370 000). This is the first study in Japan that focused on the negative impact of malpractice claims relating to diagnostic errors. In Japan, recently, attention has been directed to diagnostic errors. The diagnostic error-induced malpractice claims were as high as $37.4 \%$, which was almost similar to prior reports in the US.

Conclusion: Based on the analysis of the malpractice claims database in Japan, diagnostic errors were the main causes of medical claims and contribute negatively to many aspects.

\begin{tabular}{|c|c|c|c|c|c|c|}
\hline & & \multirow{2}{*}{\multicolumn{2}{|c|}{$\begin{array}{l}\text { Diagnostic error-related claims } \\
\qquad(n=709)\end{array}$}} & \multirow{2}{*}{\multicolumn{2}{|c|}{$\begin{array}{l}\text { Non diagnostic error-related claims } \\
\qquad(n=1093)\end{array}$}} & P-value \\
\hline & & & & & & \\
\hline \multicolumn{2}{|c|}{ Patient gender ( male \%) } & \multicolumn{2}{|c|}{$56.1 \%$} & \multicolumn{2}{|c|}{$53.0 \%$} & 0.211 \\
\hline \multicolumn{2}{|c|}{ Patient age } & \multicolumn{2}{|c|}{35 (IQR16-55) } & \multicolumn{2}{|c|}{32 (IQR 4-53) } & 0.008 \\
\hline \multicolumn{2}{|c|}{ Adjusted total billing amount } & \multicolumn{2}{|c|}{$440,000$ (IQR $185,454-775.455)$} & \multicolumn{2}{|c|}{350,909 (IQR 107,272-660,000) } & $<0.001$ \\
\hline \multicolumn{2}{|c|}{ Acceptance } & \multicolumn{2}{|c|}{$451 / 709(63.6 \%)$} & \multicolumn{2}{|c|}{$503 / 1093(46 \%)$} & $<0.001$ \\
\hline \multicolumn{2}{|c|}{ Adjusted total accepted amount } & \multicolumn{2}{|c|}{ 231,181 (IQR50,150-484,546) } & \multicolumn{2}{|c|}{136,363 (IQR 305,54-370,000) } & $<0.001$ \\
\hline \multicolumn{2}{|c|}{ Duration of claim case } & \multicolumn{2}{|c|}{$7.48( \pm 3.68)$} & \multicolumn{2}{|c|}{$7.99( \pm 4.06)$} & 0.0054 \\
\hline \multicolumn{7}{|c|}{ Clinical Feileds of malpractice claims } \\
\hline & Internal medicine & 216 & $30.5 \%$ & 208 & $19.0 \%$ & $<0.001$ \\
\hline & Obstetrics and Gynecology & 114 & $16.1 \%$ & 221 & $20.2 \%$ & 0.027 \\
\hline & Surgery & 150 & $21.2 \%$ & 189 & $17.3 \%$ & 0.04 \\
\hline & Orthopedics & 45 & $6.3 \%$ & 117 & $10.7 \%$ & 0.002 \\
\hline \multicolumn{7}{|c|}{ Outcome } \\
\hline & Deaths & 442 & $62.3 \%$ & 497 & $45.5 \%$ & $<0.001$ \\
\hline & Sequelae & 219 & $30.9 \%$ & 475 & $43.5 \%$ & $<0.001$ \\
\hline \multicolumn{7}{|c|}{ 1st Diagnosis and rank } \\
\hline 1 & Traumatic injury & 62 & $8.7 \%$ & 94 & $8.6 \%$ & 0.014 \\
\hline 2 & Malignant neoplasm & 65 & $9.2 \%$ & 85 & $7.8 \%$ & 0.291 \\
\hline 3 & Neonatal complications & 29 & $4.1 \%$ & 87 & $8.0 \%$ & 0.001 \\
\hline 4 & Procedure and postoperative complications & 14 & $2.0 \%$ & 65 & $5.9 \%$ & $<0.001$ \\
\hline 5 & Respiratory tract infection & 50 & $7.1 \%$ & 27 & $2.5 \%$ & $<0.001$ \\
\hline 6 & Ischemic heart disease & 32 & $4.5 \%$ & 40 & $3.7 \%$ & 0.366 \\
\hline 7 & Systematic infectious disease & 27 & $3.8 \%$ & 36 & $3.3 \%$ & 0.561 \\
\hline 8 & Mental disorder & 12 & $1.7 \%$ & 45 & $4.1 \%$ & 0.004 \\
\hline 9 & Non-bleeding digestive tract disease & 37 & $5.2 \%$ & 15 & $1.4 \%$ & $<0.001$ \\
\hline 10 & No abnormality & 40 & $5.6 \%$ & 8 & $0.7 \%$ & $<0.001$ \\
\hline
\end{tabular}




\title{
Clinical Vignettes
}

\section{Acute-on-Chronic Anemia Presenting as “Altered Mental Status"}

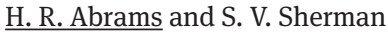 \\ Baylor College of Medicine \\ Learning Objectives: \\ 1. Describe how acute blood loss anemia leads to altered mental status. \\ 2. Recognize the limitations of exam in identifying acute upper gastrointestinal bleed (UGIB).
}

Case Information: A 59-year-old man with metastatic thoracic sarcoma complicated by encasement of the aorta presented with confusion. Two weeks prior, he had gone to another hospital for similar symptoms and improved after 2 units of blood for hemoglobin of $7.1 \mathrm{~g} / \mathrm{dL}$. He had no melena, hematochezia, or hematemesis.

His heart rate was 120, blood pressure 105/63, and other vital signs normal. He was cachectic and pale, with brown stool. His hemoglobin was $6.8 \mathrm{~g} / \mathrm{dL}$, ferritin $>1500$, and iron saturation $7 \%$. He received 3 units of blood and broad-spectrum antibiotics for a post-admission fever of 102.5. Over the next three days, he had ongoing transfusion-dependent anemia, somnolence, and tachycardia. Repeat rectal exam was normal. Full-body CT showed no focal bleeding. However, four days after admission he had melena, and EGD showed bleeding from a gastric metastasis suspected on staging CT two months prior. After endoscopic treatment, his bleeding stopped and mental status normalized.

Discussion:

- UGIB is a common inpatient condition that can be rapidly diagnosed in overt presentations. In this case, diagnosis was delayed because the patient's altered mental status was attributed to delirium, not anemia, in the absence of expected signs of UGIB and lack of reconsideration of prior imaging findings.

- Anemia causes neurological impairment and fatigue via cerebral hypoxia. Patients exposed to chronic cerebral hypoxia may be more susceptible to cognitive changes.

- The absence of melena or hematochezia on rectal exam and extremely elevated ferritin were thought to represent low likelihood of UGIB; however, exam has only $49 \%$ sensitivity for UGIB and ferritin may take several days to reflect acute changes.

\section{Cough in an Immunocompromised Adult: Same Symptoms, Different Diagnosis One Year Apart}

\section{$\underline{\text { K. Acharya }}$}

Medical College of Wisconsin

Learning Objective: Recognizing diagnostic biases and expanding the differential diagnosis (DDx) is key in coming to the correct diagnosis in immunocompromised patients.

Case Information: A 54-year-old woman presented to the clinic with persistent cough for several months. Her history was significant for systemic lupus erythematous for which she was on immunosuppression. A year prior to her presentation, she was diagnosed with Pneumocystis jirovecii pneumonia (PJP) after similar symptoms, and she had been on prophylaxis with Dapsone until recently. One month prior to her presentation, she was diagnosed with Dapsone-induced methemoglobinemia prompting a switch to Atovaquone for secondary prophylaxis; however, her cough persisted. She had several visits to the emergency room and her primary care clinic for her cough, and she was variously treated for upper airway cough syndrome and bacterial pneumonia without any improvement in her symptoms.. Her chest CT showed presence of ground-glass opacities (GGO) over several lung lobes but predominantly over the right middle lobe (RML). Given that she had a brief interruption in prophylaxis for her PJP and with similar prior radiographic features with PJP infection, she was re-treated for PJP, but her symptoms failed to improve. Eventually, she had sputum cultures checked for mycobacteria and all three samples were positive for Mycobacterium avium complex (MAC). Her symptoms resolved after she started receiving treatment for MAC infection. The total time from her presentation to achieving a correct diagnosis was four months.

Discussion: Persistent cough with presence of GGO on chest CT in an immunocompromised patient has a broad DDx. Given predominant involvement of RML, pulmonary MAC infection should have been considered as a top differential. However, the patient experienced diagnostic delay as her previous presentation led her treatment team to commit anchoring and availability biases and re-treatment of PJP infection which was far less likely than an alternate diagnosis. It was not until the DDx was expanded that the team reached the correct diagnosis. 


\title{
Hiding Around the Bend: A Missed Case of Pancreatitis
}

\author{
$\underline{\text { A. Birnbaum }}^{1}$ and A. Linker ${ }^{1}$ \\ ${ }^{1}$ Icahn School of Medicine at Mount Sinai \\ ${ }^{2}$ Division of Hospital Medicine, Mount Sinai Hospital
}

\section{Learning Objectives:}

- Analyze the causes and consequences of premature closure.

- Evaluate the potential for diagnostic error in use of specific tests for particular presenting symptoms.

Case Information: A 41 year-old woman with history of PCOS, prior DVT, and gastric sleeve presented to the hospital with abdominal pain, nausea, and vomiting. She was afebrile, hypotensive (83/52) and tachycardic (137 bpm). Physical examination revealed left upper quadrant tenderness. Labs were significant for acute kidney injury, lactate of 6, and leukocytosis. A CT abdomen/pelvis without contrast showed wall thickening and fat stranding at the splenic flexure of the colon, interpreted by the radiologist as diverticulitis. Multiple teams treated her for diverticulitis with broad-spectrum antibiotics and fluids for 5 days with incomplete resolution of symptoms. A repeat CT scan with IV contrast revealed fat stranding of the pancreas with extension to the right paracolic gutter and a portal vein thrombus, consistent with pancreatitis, without evidence of diverticulitis or abscess. She was treated with dietary restriction and fluids, and symptoms resolved. Review of the history and workup revealed a lipase of 569 on admission that was not acknowledged in documentation. Her risk factors for pancreatitis included heavy alcohol use (discovered after pancreatitis diagnosis) and GLP-1 agonist use for PCOS.

Discussion: In this case, clinicians prematurely closed upon a diagnosis of diverticulitis following radiological interpretation of a CT scan that was limited by lack of IV contrast in the setting of AKI. The patient met criteria for pancreatitis on admission, before imaging, and the elevated lipase should have raised the pre-test probability for pancreatitis. This case demonstrates the importance of re-evaluation and review of clinical data when assuming care of a patient, and the need to consider the validity of a study (CT without contrast for evaluation of pancreatitis) when considering diagnostic certainty.

\section{Missed Connection: Imperfect Role of Imaging in Diagnosis of Esophagopleural Fistula}

\author{
E. M. Cherry and J. N. Lessing \\ University of Colorado School of Medicine
}

\section{Learning Objectives:}

1. Imaging limitations in the diagnosis of esophagopleural fistula

2. Role of nonoperative management of esophageal perforation and effusion

Case Information: A 37 yo man presented with sudden food impaction. After endoscopic disimpaction, CT chest with oral contrast revealed perforation at the GE junction, extraluminal contrast in the Left paraesophageal space, and pneumomediastinum. After emergent endoscopic esophageal stenting, repeat CT chest demonstrated an unexplained Left pleural effusion for which a percutaneous thoracostomy was placed. Plain esophagram did not demonstrate stent leak.

Medical management consisted of strict NPO, intravenous fluids, IV PPI, broad-spectrum antibiotics, and thoracostomy drainage. After 5 days, repeat plain esophagram was negative for esophagopleural fistula. CT chest demonstrated decreased Left effusion volume, but interval development of pneumohydrothorax. Video-Assisted Thoracic Surgery decortication was converted to open thoracotomy due to discovery of food matter in the pleural space.

Discussion: This case highlights limitations of advanced imaging and implications for nonsurgical management of esophageal perforation. Esophageal rupture is a surgical emergency, though nonoperative management is emerging as a viable alternative to high-morbidity and high-mortality surgical interventions. Initial management includes NPO, hemodynamic monitoring, surgical evacuation of extra-mediastinal leaks, esophageal stenting, and broad-spectrum antibiotics. (PMID: 16427833, PMID: 25791945)

Chest CT is the preferred imaging modality for stratifying pleural space infections, though esophagopleural fistulas may not be apparent on imaging, especially within the first 24 hours, and can occur irrespective of radiographic mediastinitis (PMID: 3490162, PMID: 20133295, PMID: 28575240). An esophagopleural fistula was not demonstrated on the initial CT prior to esophageal stenting, nor in subsequent CTs and esophagrams. However, vegetable discovered in the pleural space during VATS indicated definite transesophageal migration of food into the pleural space. The esophageal rupture was not contained to the mediastinum and could not be successfully managed nonoperatively. Understanding the limitations of radiology in diagnosing an esophagopleural fistula is essential to considering this diagnosis. Diagnostic performance of CT scan for esophageal perforation is in Table 1. In retrospect, a higher clinical suspicion was warranted for esophagopleural fistula as a cause for the effusion. Surgical intervention was delayed because of negative imaging. Meanwhile, the patient clinically 
deteriorated. Eventual development of hydropneumothorax necessitated decortication. His complete recovery validated surgical intervention for pleural effusion with distal esophageal perforation.

Table 1. Performance of CT esophagram findings for suspected esophageal rupture.

\begin{tabular}{lcccccc}
\hline \multicolumn{1}{c}{$\begin{array}{c}\text { Radiographic } \\
\text { Finding }\end{array}$} & $\begin{array}{c}\text { Sensitivity } \\
(\%)\end{array}$ & $\begin{array}{c}\text { Specificity } \\
(\%)\end{array}$ & $\begin{array}{c}\text { PPV } \\
(\%)\end{array}$ & $\begin{array}{c}\text { NPV } \\
(\%)\end{array}$ & +LR & -LR \\
\hline $\begin{array}{l}\text { Esophageal wall } \\
\text { discontinuity }\end{array}$ & 55.6 & 96.3 & 71.4 & 92.9 & 15 & 0.46 \\
$\begin{array}{l}\text { Esophageal wall } \\
\text { thickening }\end{array}$ & 33.3 & 94.4 & 50 & 89.5 & 5.9 & 0.71 \\
$\begin{array}{l}\text { Leakage of contrast } \\
\text { into mediastinum or } \\
\text { pleural space }\end{array}$ & 55.6 & 96.3 & 71.4 & 92.9 & 15 & 0.46 \\
$\begin{array}{l}\text { Extraluminal air or } \\
\text { fluid collections }\end{array}$ & 66.7 & 94.4 & 66.7 & 94.4 & 11.9 & 0.35 \\
$\begin{array}{l}\text { Left pneumothorax } \\
\text { or pleural effusion }\end{array}$ & 55.6 & 66.7 & 21.7 & 90 & 1.7 & 0.67 \\
$\begin{array}{l}\text { Pneumomediastinum } \\
\begin{array}{l}\text { Marked esophageal } \\
\text { fistula }\end{array}\end{array}$ & 44.4 & 75.9 & 23.5 & 89.1 & 1.8 & 0.73 \\
\hline $\begin{array}{l}\text { Table adapted from a ross-sectional analysis of CT esophagography compared to } \\
\text { surgical inspection by Suarez-Poveda et al. (Emergency Radiology 21:505-510, 2014) }\end{array}$
\end{tabular}

\section{A "Rheumination" on Failure to Thrive}

C. Duarte, J. Zoucha and J. Lessing

The University of Colorado

\section{Learning Objectives:}

- $\quad$ To understand the clinical and laboratory manifestations of ANCA-associated vasculitis

- To understand how diagnosis momentum and premature closure affect diagnostic reasoning

Case Information: A 70-year-old man with history of MPO+/ANCA-associated vasculitis, pulmonary sarcoidosis, and CKD was hospitalized for weight loss and "hurting all over." Aside from baseline 2L oxygen requirement, physical exam was normal. Labs were pertinent for a sodium of 133, creatinine of 2.6 (prior baseline 1.7), WBC 14, hemoglobin 10.2 (prior 14), and platelets 850 . Urinalysis demonstrated 2+ protein, 2+ blood, 20-50 RBCs. P-ANCA levels were 1:640 (prior <1:20). The admitting team's differential diagnosis included flare of vasculitis or sarcoidosis, and failure to thrive (FTT). However, hand-off between teams focused on FTT and depression, as opposed to noting his symptoms and organ dysfunction. This prompted a work-up for endocrine causes of weight loss and fatigue, cancer screening, and pulmonary/nephrology consultations, who stated his chronic conditions were stable. Unexplained fevers and tachycardia persisted. Five days later with little clinical improvement, the decision was made to consult rheumatology. Based on physical exam and labs (hematuria, AKI, increased p-ANCA titers) rheumatology suggested active vasculitis as the diagnosis.

Discussion: This case highlights multiple issues in diagnostic reasoning. First is absent knowledge of features suggesting active vasculitis. Second is diagnosis momentum and premature closure. Admitting providers anchored on depression and FTT as final diagnoses despite lab evidence that the patient had organ dysfunction. The patient was handed off as a case of FTT, making it difficult to consider anything else. FTT is becoming a common admission diagnosis for patients; however, it is imprecise and takes focus away from the patient's symptoms, prematurely stopping the diagnostic search, implying an irreversible state due to chronic disease. Further research on FTT as an admission diagnosis and whether or not it affects diagnostic workup should be pursued. 


\title{
Using the EMR to Hunt Zebras
}

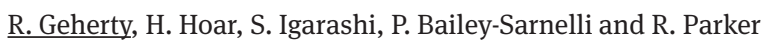
UMMS-Baystate

Case information: XR is a 20 year old woman with a history of substance abuse and remote removal of a mature ovarian dermoid cyst. She presented twice within one week with altered mental status. On initial presentation, she was diagnosed with acute anxiety and discharged home. She returned with similar symptoms attributed to recreational drugs. She was admitted to the ICU for frequent monitoring with an admission diagnosis of acute encephalopathy secondary to substance abuse. On hospital day 2, a message was sent from her gynecologist who had removed her ovarian teratoma suggesting anti-NMDA encephalitis, which was ultimately confirmed as her diagnosis.

Discussion: Early prompting of the primary team shortened the path to reaching this rare diagnosis. Substance abuse is a common and readily blamed cause of encephalopathy. In addition, return visits for the same complaint should prompt diagnosticians to reconsider the diagnosis. Years prior, her gynecologist had enabled an EMR flag to notify her whenever this patient presented to the hospital. In this case, the return visit for apparently psychiatric complaints is what led the specialist to become concerned. This case illustrates the importance of communication between specialists, and the possibility of the EMR to facilitate this. There are other examples in the literature of recognition algorithms improving diagnosis when embedded within the EMR, but most relate to common but easily-overlooked diseases. This ability to communicate to other teams via EMR is novel and likely underutilized. Combined with the insight that another specialty may not be aware of certain disease associations, difficult diagnoses can be made much simpler.

\section{Importance of Imaging in Knee Pain}

$\underline{\text { E. J. Gibson }}^{1}$, P. Mukkamala ${ }^{1}$, L. Lopez ${ }^{2}$, T. M. Goldson ${ }^{1}$ and S. N. Forjuoh ${ }^{1}$

${ }^{1}$ Family \& Community Medicine, Baylor Scott \& White Health and Texas A\&M Health Science Center College of Medicine, Temple, Texas ${ }^{2}$ Pathology, Baylor Scott \& White Health and Texas A\&M Health Science Center College of Medicine, Temple, Texas

Joint complaint is very common in the primary care setting. In the United States, an estimated 126.6 million people suffer from a musculoskeletal disorder, reflecting an economic burden with an estimated cost of $\$ 213$ billion annually. Knee pain is the second most prevalent condition and patellofemoral pain is considered one of the most common forms of knee pain. A 72-year-old Caucasian woman with prior history of stage IIIa adenocarcinoma of the lung was seen with complaints of knee pain, swelling, and difficulty sleeping at night for one month. Although mimicking minor issues such as osteoarthritis, patellofemoral syndrome, or iliotibial band syndrome, imaging was ordered, particularly due to her history of lung cancer and melanoma. X-rays showed lytic lesion within the proximal meta-epiphyseal region of the right tibia, suspicious of metastatic disease. Her treatment involved excision of the right tibial lesion and saphenous neurolysis. Once completed, she received radiation treatment, along with four cycles of chemotherapy and did very well. This case represents the importance of early imaging in patients with knee pain of prolonged duration seen in the primary care setting.

\section{"Altering the Differential"}

\author{
C. A. Iqbal and R. Rana
}

Baylor College of Medicine

\section{Learning Objectives:}

- $\quad$ Develop a robust, inter-disciplinary differential diagnosis for acute altered mental status

- Consider alternative etiologies for such a presentation when confronted with diagnostic uncertainty

Case Information: We present the case of a 79-year-old woman with no known medical history brought in by her daughter for the acute onset of altered mental status. Her symptoms progressed from initially speaking in incoherent phrases and declining food, to ultimately only groaning, urinating on herself, and lethargy. Her cognitive baseline was that of a completely functional woman, who, apart from mild physical debility, would perform activities of daily living with minimal assistance.

Initial workup, including CBC, CMP, ammonia, B12, RPR, TSH, urinalysis, urine culture, chest radiograph, and non-contrast CT of the head were all negative. Though initially unremarkable, review of overnight vitals from her first night of admission revealed profoundly labile heart rates and blood pressures, with bradycardia paired with hypotension alternating with tachycardia paired with hypertension. Ultimately, reconsidering the differential diagnosis in clinical context led to obtaining an MRI of the brain, revealing an acute ischemic infarct of the left perisylvian region involving the inferior temporal lobe and insula. 
Discussion: This case highlights the profound importance of several key tenets of diagnostic reasoning. Correctly characterizing presenting symptoms into an accurate "problem representation", systematically evaluating a differential diagnosis for a given illness script, avoiding premature closure, and the importance of an interdisciplinary knowledge base beyond what one typically encounters in one's own clinical practice were all critical in ultimately arriving at the correct unifying diagnosis. Furthermore, this case provides a fascinating platform to review the relevant neuroanatomy and neurophysiology underlying dysautonomia and aphasia, as well as an opportunity for insight into an approach to altered mental status, including potential diagnostic pitfalls encountered in working up an essential clinical presentation.

\title{
Confused and Confusing: Thrombocytosis as an Unexpected Cause of Encephalopathy
}

\author{
C. Kappauf $^{1}$ and A. Linker ${ }^{2}$ \\ ${ }^{1}$ Icahn School of Medicine at Mount Sinai \\ ${ }^{2}$ Mount Sinai Department of Medicine, Division of Hospital Medicine
}

\author{
Learning Objectives: \\ - Consider thrombocytosis as a cause of encephalopathy in patients with essential thrombocythemia (ET) \\ - Correcting thrombocytosis may improve encephalopathy in patients with ET \\ - Evaluate treatment thresholds while working through a diagnostic dilemma
}

Case Information: A 44-year-old woman presented to the ER with two weeks of headache, confusion and abnormal behavior. Medical history included remote traumatic brain injury with cognitive impairment, depression, type 2 diabetes, chronic idiopathic pancreatitis with recent distal pancreatectomy/splenectomy, treated late latent syphilis and frequent marijuana use. On presentation, she was afebrile, hypertensive, oriented only to name and intermittently agitated. She was recently admitted to another hospital where workup included labs (showing thrombocytosis) and head imaging. Jak-2 testing returned positive after discharge. She presented to our hospital with persistent encephalopathy. Testing on admission showed thrombocytosis (938,000/uL), leukocytosis (17,600/uL, 11\% neutrophils), hyperglycemia (279) and proteinuria. Infectious workup (HIV, RPR, blood cultures, lumbar puncture) was unrevealing. Head CT, MRI brain with MRA and MRV were negative. Given the isolated thrombocytosis, hydroxyurea and aspirin were initiated. The platelet count decreased to 597,000/uL with a corresponding resolution of encephalopathy. She was discharged and continues to do well on hydroxyurea and aspirin.

Discussion: This case presented a diagnostic challenge. Multiple comorbidities could have contributed to her mental status change. The team considered a wide differential diagnosis - infection, venous sinus thrombosis, hypertensive emergency, neurosyphilis, substance-induced psychosis - but workup was negative, prompting consideration of less common diagnoses, such as thrombocytosis-induced microvascular changes. We weighed the risk/benefit of initiating ET treatment while the workup was ongoing. Though ET-related delirium has only been reported once, this patient's marked clinical improvement with correction of her platelet count and little other intervention suggests that significant thrombocytosis contributed to her encephalopathy. Patients with lower cognitive reserve may be at higher risk of decompensation in the setting of Jak2-associated thrombocytosis.

\section{Be Careful about the Perianal Pain! Fournier’s Gangrene}

\author{
T. Kawahigashi ${ }^{1}$ and K. Watanabe ${ }^{2}$ \\ ${ }^{1}$ Department of Emergency Medicine, Tokyo West Tokushukai Hospital \\ ${ }^{2}$ Department of Surgery, Tokyo West Tokushukai Hospital
}

Learning objectives: Fournier's gangrene is an uncommon disease, but any delay in treatment could be lethal.

Case information: A 69-year-old Japanese man noticed anal pain five days before his first visit. Although he had been aware of perianal tiny mass for a long time, he had left it because it had not shown any symptoms. Subsequently, he developed a high fever and visited our hospital. On examination, he had a fever of 39.5C, but other vital signs were within normal limits. $5 \mathrm{~mm}$ perianal mass located in the 12 o'clock positions, but it showed poor signs of inflammation such as redness, heat, and swelling. It was initially diagnosed infected epidermal cyst because of previously noticed mass and recent fever and pain. We asked him to visit a surgical department the next day because the anal findings were mild. On the following day, the surgeon performed plan abdominal computed tomography scan, which showed abscess containing fluid and air in the subcutaneous tissues of perianal regions. He was diagnosed as Fournier's gangrene and treated with immediate extensive surgical treatment. He also received broad-spectrum antibiotics. Subsequently, healthy granulation was present in the wound in several days after the surgery. He was finally discharged on the 11th postoperative day.

Discussion: Fournier's gangrene, a fulminant form of infective necrotizing fasciitis of the perineum and genital area, is a rare but medical emergency. It requires urgent surgical debridement as well as the proper antibiotic treatment. However, it is sometimes difficult because of its rarity and paucity of skin findings early in the disease. Although he complained of severe anal pain, we missed this life-threatening condition at present due to poor inflammation signs and premature closure. Physicians need to have a high level of awareness of this disease when they encounter patients with severe genital or perianal pain. 


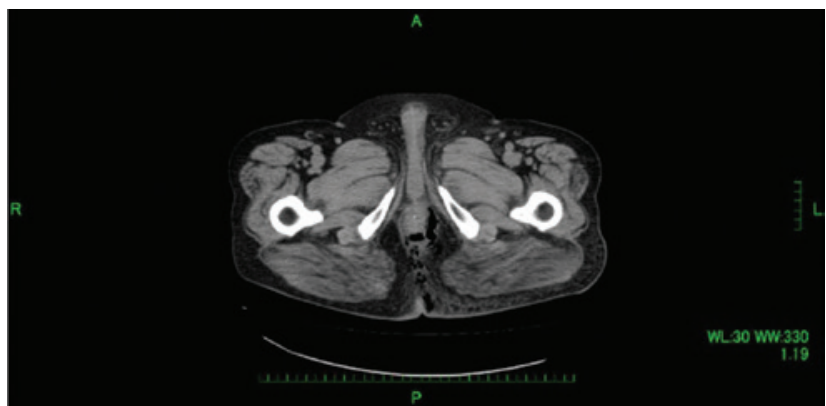

Careful History-Taking in Developmentally Delayed Patients with Altered Mental Status

\section{A. Kleinman}

Akron Children’s Hospital, Akron, Ohio.

Learning objective: Identify patients with history of altered mental status at high risk for occult infection and underlying pathology for who focused history-taking is essential, even without a focal physical exam or ongoing symptoms.

Case Information: A sixteen year-old male with Simpson-Golabi-Behmel syndrome, developmental delay, chronic kidney disease, autistic spectrum disorder, and history of primary hepatoblastoma presented to the pediatric Emergency Room for "not acting right" during gym class in school. After prolonged exercise, patient became unwilling to walk and diaphoretic with pronounced facial pallor. He had no loss of consciousness. At the time, he complained of left lower quadrant abdominal pain and nausea. Family was called by the patient's teacher and father noted that patient was ill-appearing on his arrival.

In the ER, patient was back to baseline energy and mentation with no active complaints. He denied persistent abdominal pain or nausea. Exam was negative for changes in mentation or energy level with a nonfocal neurologic exam. The abdomen showed no distension, tenderness to palpation, or rebound. Given the patient's extensive past history and developmental delay, however, more detailed history was obtained. Directed questioning confirmed that given patient's autism and intellectual disability, he had a history of ingesting inorganic material. Despite lack of focal abdominal exam, x-ray of abdomen was obtained with findings concerning for small bowel obstruction (SBO) with air fluid levels and questionable gastric bezoar noted. Pediatric surgery was consulted with CT confirmation of SBO; patient was taken to OR where he underwent a diagnostic laparoscopy that required conversion to laparotomy with lysis of previous surgical adhesions. After a prolonged recovery, patient was discharged home on post-operative day \#8.

Discussion: The differential diagnosis for pediatric altered mental status is broad- spanning multiple organ systems and ranging from nonaccidental trauma and ingestion to benign fatigue. Patients with intellectual disability and limited communication ability require even wider consideration given their proclivity for age-inappropriate behavior and their increased risk for abuse. As a result, detailed history with attention to possible sources of injury and underlying disease are essential. Even in the setting of a reassuring physical exam and resolution of initial complaint, a high index of suspicion should be maintained for ongoing pathology. The risk for errors in clinical reasoning and delayed diagnosis is especially elevated in this high-risk population, necessitating extra vigilance and attention to detail. 


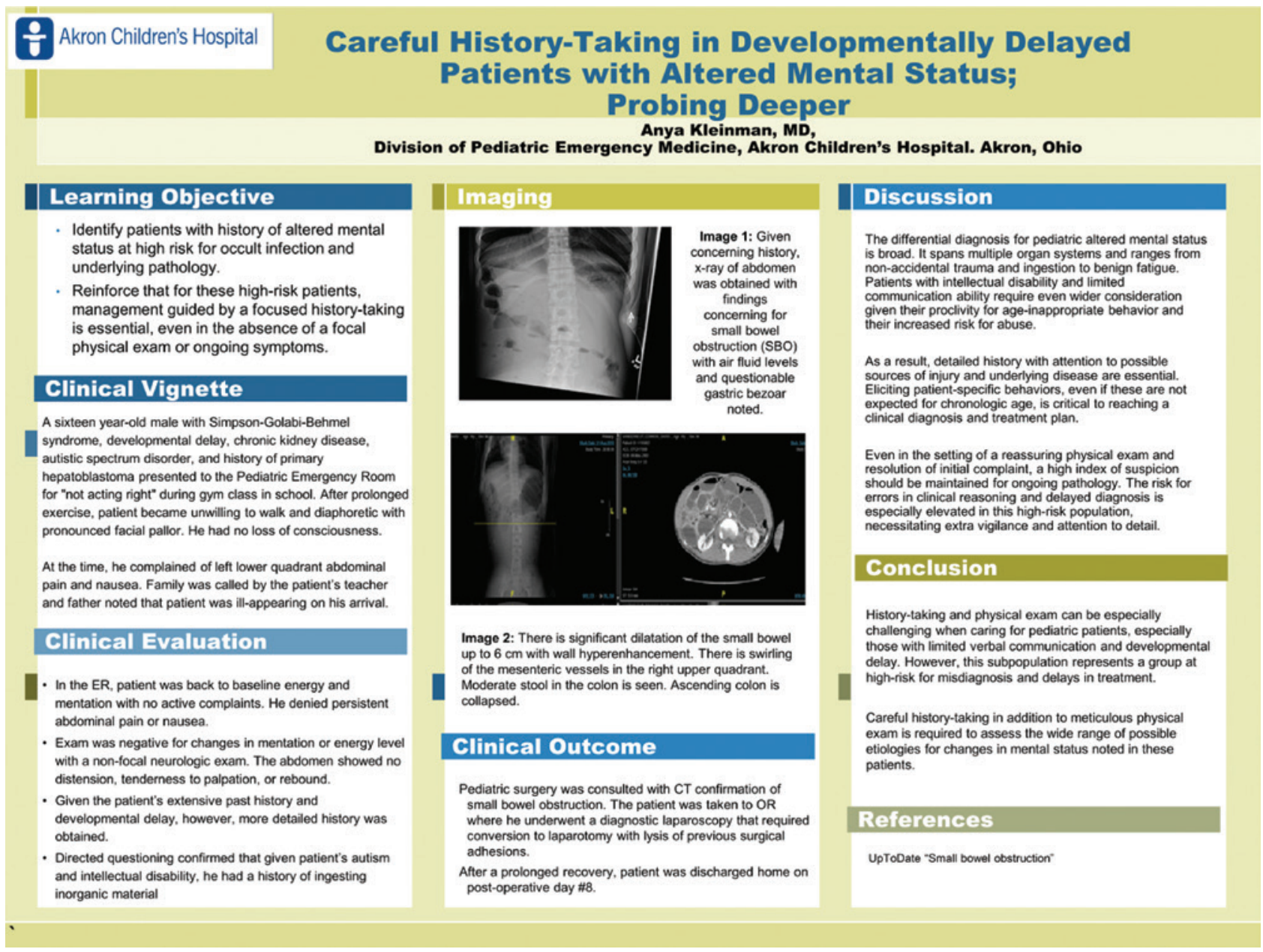

\title{
A Masquerading Chest Mass
}

\author{
$\underline{\text { A. Kleinman }}$ \\ Akron Children's Hospital, Akron, $\mathrm{OH}$.
}

Case Presentation: 10 year old female presented to her pediatrician with lateral, right chest wall swelling. History was negative for trauma, fever, and other areas of swelling or lymphadenopathy. Patient had no personal or family past history of skin or soft tissue infections. She was diagnosed with acute lymphadenitis and prescribed Bactrim. 4 days later, she re-presented to the Pediatric Emergency Room with an enlarging lesion and worsening pain with pressure on affected side, especially when recumbent. No clinical improvement had been noted with consistent Bactrim treatment, yet patient had continued to be afebrile. She now, however, endorsed increased fatigue and decreased appetite. ER physical exam noted a right anterior axillary lesion, $3 \times 2 \mathrm{~cm}$ with bluish-yellow discoloration and a $1 \mathrm{~cm}$ mobile, indurated, tender mass. In the absence of systemic symptoms, no bloodwork was obtained. Diagnostic evaluation was significant for US finding of $1.8 \mathrm{~cm}$ complex subcutaneous cyst in the right lateral chest. Patient was diagnosed with a lymphatic malformation after consultation with plastic surgery. She was referred to the vascular anomalies clinic. MRI outpatient re-demonstrated the subcutaneous lesion without involvement of deeper structures. The patient was prescribed symptomatic therapy with ibuprofen as needed. Sclerotherapy was planned if symptomatic treatment with NSAIDS was unsuccessful.

Discussion: Lymphatic malformations are rare congenital vascular anomalies that have heterogeneous presentations. They can often masquerade as other, more common, diagnoses, such as cellulitis, lymphadenitis, and abscess. It is important to be aware of the natural history of lymphatic malformations as well as their evaluation and management; a high index of suspicion is required for this uncommon medical diagnosis. This patient's lack of fevers and failure to improve on appropriate treatment for lymphadenitis were red flags for the less common diagnosis of lymphatic malformation. Careful history and physical exam were crucial to avoiding a misdiagnosis and delay in treatment in this case. Conclusion: The prevalence of lymphadenitis, cellulitis, and abscess is ever increasing with growing MRSA prevalence. Yet, it is imperative for physicians to remain alert to uncommon diagnoses, especially with unusual clinical presentations and/or clinical course. 


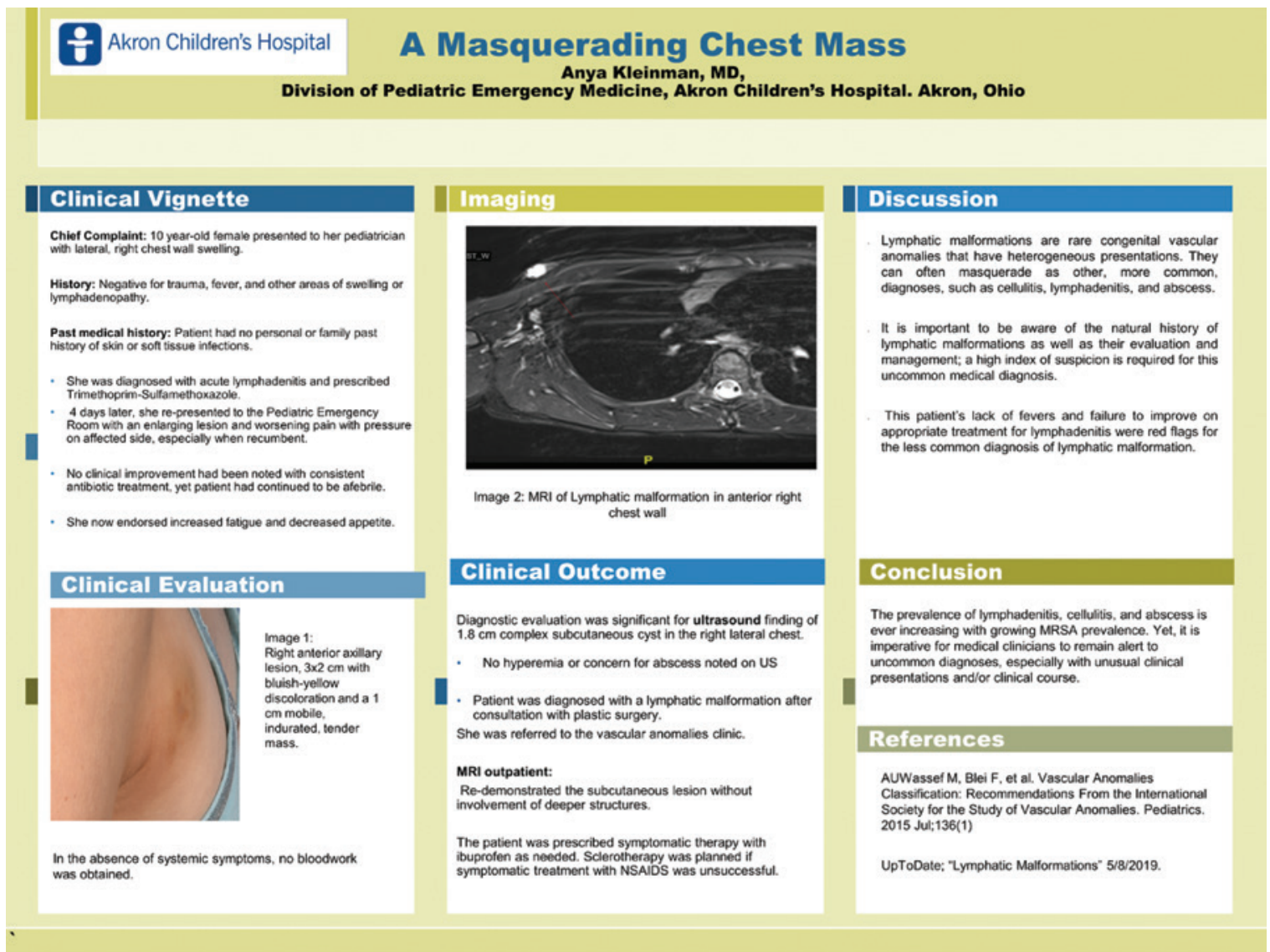

\section{Delayed Diagnosis in a Neonatal Coarctation Presenting with Respiratory Distress to a Community Hospital}

\section{$\underline{\text { W. Lilitwat }}{ }^{1}, \mathrm{~K}$. Wellnitz ${ }^{2}$ and C. Tigges ${ }^{2}$}

${ }^{1}$ Texas Tech University Health Science Center, Lubbock

${ }^{2}$ University of Iowa Stead Family Children's Hospital

Learning objectives: Understand bias such as availability, anchoring, and satisfaction of search, contribute to diagnostic error.

Case information: A 16 day-old term, male infant presented to ER at a community hospital with one day history of respiratory distress. The afebrile infant appeared pink but SpO2 84\%, RR in 40, HR 171, and BP 97/44. SpO2 improved to 96\% on 0.25 LPM NC (FiO2 1). Labs were normal. Chest radiograph was interpreted as right upper lobe infiltration. Based on the history of contact with RSV, bronchiolitis was the working diagnosis. During ground transport to our tertiary care facility, he developed shock with BP 40s/20s. At our ED, vigilant review of his chest radiograph revealed cardiomegaly. He was subsequently started on inotropic support and intubation was performed. Echocardiography showed critical coarctation. Patient subsequently underwent coarctation repair and was discharged home.

Discussion: This case illustrates an example of delayed diagnosis, revealing the process of a typical cognitive error: availability, satisfaction of search and anchoring. The community hospital inferred respiratory distress as bronchiolitis because of respiratory symptoms. Additionally, radiographic lesions remain undetected after detection of an initial lesion-satisfaction of search. The transport team was also anchored to the diagnosis of bronchiolitis. However, the true diagnosis was the critical heart disease. In a case of a neonate presenting with respiratory distress and shock, it is important to include congenital heart disease as a part of the differential diagnosis and avoid anchoring. 


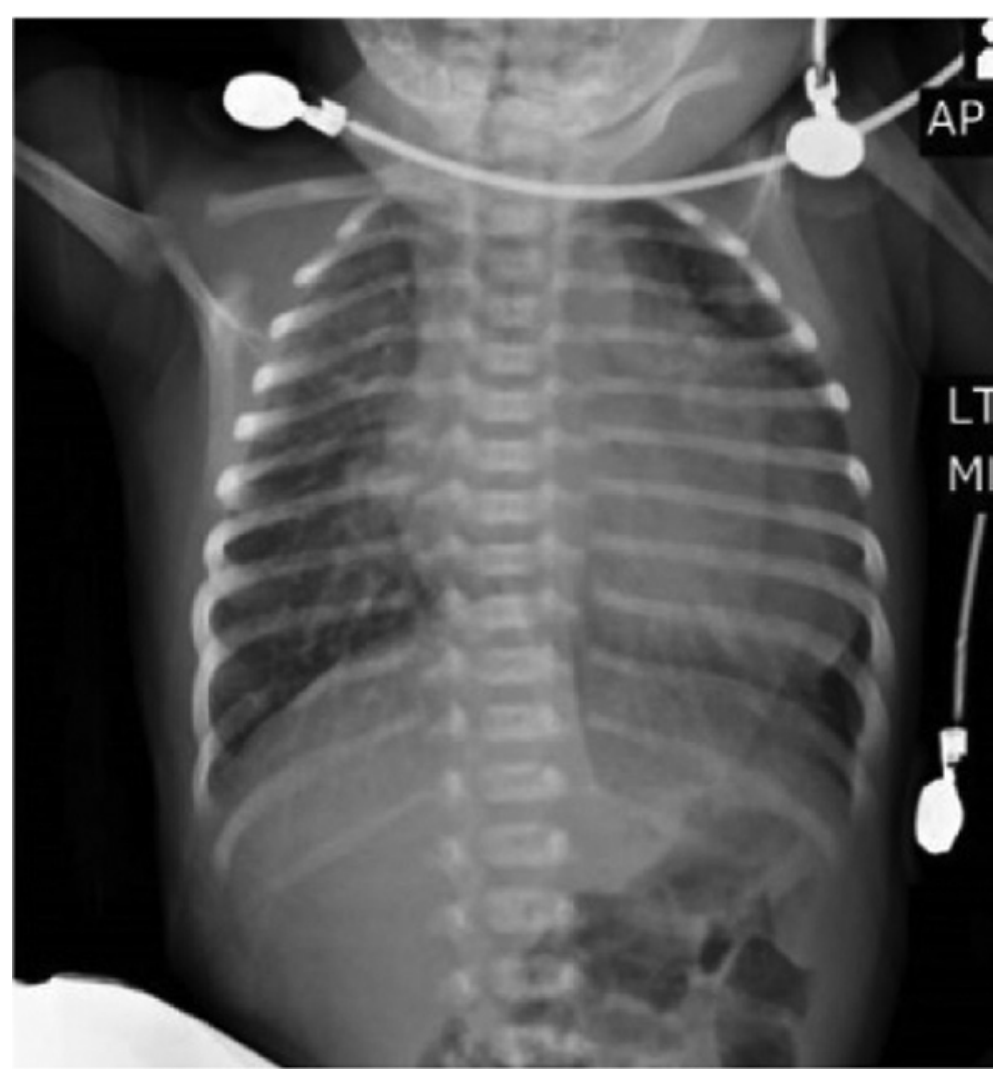

\title{
Hands-on Handoffs: Patient Care Transfers as Opportunity to Revisit a Diagnosis in a Patient with Anti-MDA5 Rapidly Progressive Interstitial Lung Disease
}

\author{
N. Mistry and J. N. Lessing \\ University of Colorado School of Medicine
}

\section{Learning objectives:}

1. Identify anti-melanoma differentiation-associated 5 (MDA5)-antibody positive clinically amyopathic dermatomyositis as a potential cause of rapidly-progressive interstitial lung disease

2. Recognize that loss of information is common with handoffs

3. Label 2 cognitive biases

Case Information: A 35-year-old man hospitalized for 3 weeks of progressive dyspnea and chest x-ray interpreted as pneumonia rapidly developed respiratory failure requiring ventilation and ECMO. Because of groundglass opacities and hilar lymphadenopathy seen on CT, serologic work-up for underlying rheumatologic conditions was sent. In the setting of multiple team handoffs during a 2-week lag for sendout tests, the positive anti-MDA-5 antibody result was not immediately recognized when resulted. These results suggested rapidly-progressive interstitial lung disease (RP-ILD) with clinically amyopathic dermatomyositis (CADM), necessitating aggressive immunosuppression. His condition worsened despite treatment, and he died 8 weeks later from cardiopulmonary arrest.

Discussion: Anti-MDA-5 antibody is a marker of CADM, a subset of dermatomyositis. Patients with this condition have characteristic cutaneous ulcerations and are at high risk of RP-ILD, portending poor survival. However, pulmonary disease can precede CADM-associated skin findings, as this patient lacked cutaneous features. Treatment involves aggressive immunosuppression.

Not uncommon for rare diseases, the correct diagnosis was delayed. However, in this case, the necessary test was sent, but not seen. During his prolonged hospitalization, his care was handed off between multiple teams, likely leading the providers to overlook the myositis panel results from weeks earlier. Search satisfaction (initial imaging showed pneumonia and possible sarcoidosis), anchoring and momentum (new teams continuing prior teams' treatments) all likely contributed to the delay.

When caring for a patient with RP-ILD, consider anti-MDA5 CADM as a cause, keeping in mind pulmonary disease may precede other systemic findings. This sad case teaches us to use handoffs as an opportunity to be hands-on about actively revisiting assumptions and reviewing the medical record. 


\title{
Overmine a cause of a mineral metabolism disorder. A near-miss premature closure of hypercalcemia case due to a conspicuous medication history
}

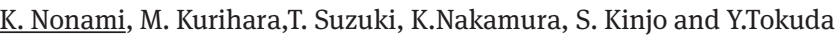 \\ Department of Hospital Medicine, Urasoe General Hospital \\ Muribushi Project for Teaching Hospitals
}

Learning objectives: Reveal a correct diagnosis masked by cognitive biases through re-calibrating a diagnostic process.
Case information:

An 80-year-old man with a history of mental disorder presented with a two-week history of malaise and constipation that several prior physicians could not identify their cause. Medication history revealed that he had taken a calcium supplement and following blood test revealed severe hypercalcemia $(16.8 \mathrm{mg} / \mathrm{dL})$ and the most likely etiology of hypercalcemia was considered with possible overdosing of calcium supplement or milk-alkali syndrome. Thus a closed case was suggested, and the patient discharged without any symptom after collection of serum calcium. However, increased intact parathyroid hormone (PTH) and a normal value of PTH-relating protein decreased the possibility of the prior diagnosis, and the patient had been under further follow-up at our outpatient clinic. Thorough history taking, physical examination, and imaging studies excluded a diagnosis of metastatic bone cancer or primary bone cancer and confirmed an accumulation of 18F-fluorodeoxyglucose at right large parathyroid. Eventually, a diagnosis of primary hyperparathyroidism possibly aggregated by calcium supplement was made.

Discussion: In our case, although major etiologies in hypercalcemia were hyperparathyroidism or malignancy-related hypercalcemia, medication history misled us to a wrong diagnosis of substance-induced hypercalcemia, incompatible with a prevalence of the disease. However, further testing re-calibrated our working diagnosis and eventually succeeded to make the correct diagnosis. An outstanding diagnostic clue can have a risk of availability bias, which leads a diagnosis to that of easily come to mind and sometimes results in making a wrong diagnosis. For avoiding such heuristics and subsequent misdiagnosis caused by conspicuous clues, physicians need to re-calibrate their diagnostic process through monitoring of information including signs and symptoms or laboratory values which inconsistent to working diagnosis or evoking alternative diagnoses.

\section{Can’t See, Can’t Pee, Can’t Climb a Tree is Not Always Reit}

L. Pell and E. Szemak

University of Colorado Hospital

\section{Learning objectives:}

- $\quad$ Understand the epidemiology of reactive arthritis after genitourinary or enteric infections.

- Develop strategies to avoid framing and confirmation bias during handoffs

- Understand the epidemiology of ocular manifestations of systemic lupus erythematosus

Case Information: 25 year old African American female with past history of gonococcal cervicitis presented with decreased vision. This progressed over 6 weeks and was associated with sinus pressure treated with penicillin 2 weeks ago with no improvement, so she presented to an emergency room. During this period, she noted some burning with urination and swelling and pain in her right knee. She denied recent unprotected sex and there was no recent knee trauma. In the emergency room she had mild leukopenia, hypoalbuminemia, and proteinuria but otherwise had a normal comprehensive metabolic profile, complete blood count, and dipstick urinalysis. She was admitted to the hospital due to inability to ambulate and suspected new diagnosis of reactive arthritis with visual disturbance, knee swelling, and dysuria. Ophthalmologic exam on admission revealed normal slit lamp, retinal, and acuity exam. Visual complaints were attributed to periorbital edema. Urine studies reveal 1.3g/day proteinuria. Serum C3 and C4 were low, and she had a high serum ANA and DS-DNA titer. Gonococcal and chlamydial urine gene studies were negative as was serum HIV ELISA assay. Renal biopsy demonstrated glomerular deposits of IgG, IgM, IgA, C3, and C1q with active mesangial glomerulonephritis. New diagnosis of systemic lupus erythematosus with active glomerulonephritis was made and treatment initiated.

Discussion: Reiter's Syndrome is the former name for a subtype of reactive arthritis that presents with the triad of arthritis, urethritis, and conjunctivitis and is HLA-B27 associated. This subtype presents with all 3 symptoms in less than $2 \%$ of patients ultimately diagnosed with seronegative spondyloarthropathies. These symptoms present 2-3 weeks after a genitourinary or enteric infection, with the most common causative agents being Chlamydia trachomatis or Salmonella species respectively. The HLA-B27 haplotype associated with this disease is more than twice as common in white vs. black Americans. 
One-third of patients with systemic lupus erythematosus have ocular manifestations with the most common being keratoconjunctivitis. Retinal vasculitis, optic neuritis/neuropathy, and periorbital edema are seen in $<2 \%$ of initial presentations.

With lack of clinical experience with rare conditions, we often fall back on clinical mnemonics that we learn during training. It is helpful to reframe cases for yourself whenever assuming care of patients with new rare diagnoses.

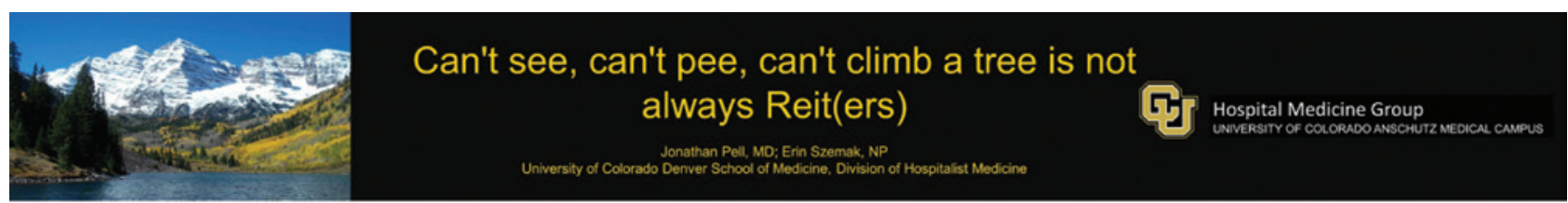

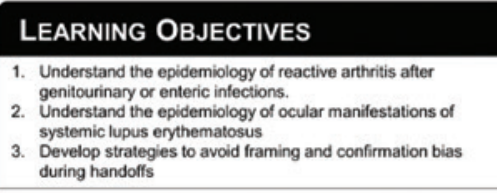

\section{CASE}

HISTORY

25 year old African American female with past history of gonococcal cervicitis 5 yrs ago presented with decreased vision. This progressed over 6 weeks and was associated with sinus pressure so treated with penicillin 2 weeks prior to current presentation with no improvement, so presented to the hospital. During this period, she noted some burning with urination and swelling and pain in her right knee. She denied recent unprotected sex and there was no recent knee trauma. She was admitted to the hospital due to inability to ambulate and suspected new diagnosis of reactive arthritis with visual disturbance, knee swelling, an dysuria

EXAM: Temp 38.1" C. HR 94, BP108/75, RR16, 94\% on RA Eyes- normal slit lamp, retinal exam, bilat periorbital edema Musculokeletal - bilat knee effusions

LABS: WBC 3.2 Albumin $2.2 \mathrm{mgdL}$

U Protein/U Create: $295 / 179$

C3 $26 \mathrm{mg} / \mathrm{dL}$ C4 $<8 \mathrm{mg} / \mathrm{dL}$

ANA $>1: 320$

Double-Stranded DNA $>300$ units

Gonococcal and chlamydial urine

PCR - negative

Renal biopsy IF- glomerular

deposits of $1 \mathrm{gG}$, IgM, IgA, C3, and

deposits of $\lg \mathrm{G}, \operatorname{lgM}, \lg A, \mathrm{C} 3$, and

C1q with active mesang

glomerulonephritis

isteributed to periorbital edema. High cere initiated for end organ damage of renal disease and central nervous system involvement

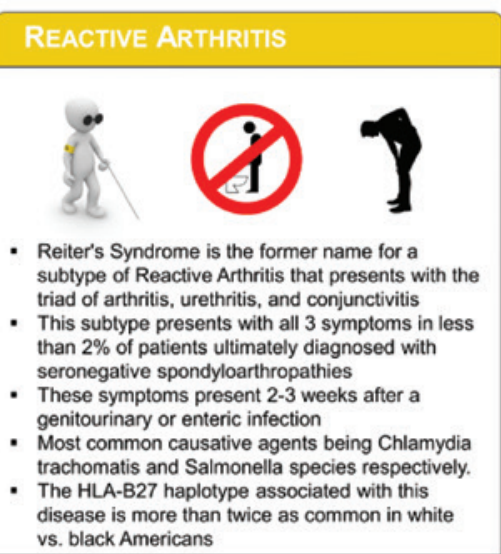

\section{SYSTEMIC LUPUS ERYTHEMATOSUS}

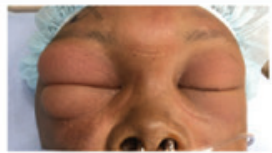

- One-third of patients with systemic lupus erythematosus have ocular manifestations with the most common being keratoconjunctivitis

- Retinal vasculitis, optic neuritis/neuropathy, and periorbital edema are seen in $<2 \%$ of initial periorbital edemations

presentations
- $8 \%$ of women with systemic lupus erythematosus have lower urinary tract symptoms

\section{Discussion}

- Reactive arthritis in an African American female from a gonococcal urethritis that occurred 5 years in the past would be exceedingly rare

Mnemonics supporting checklists can decrease cognitive bias

- Mnemonics for recalling manifestations for rare diseases are at high risk of confirmation bias and satisfied search particularly in the less experienced clinician

Clinicians are more likely to see rare presentations of common diseases than common manifestations of rare diseases

- Use of heuristics during high workload times can lead to mnemonic based biases and resultant framing during handoffs
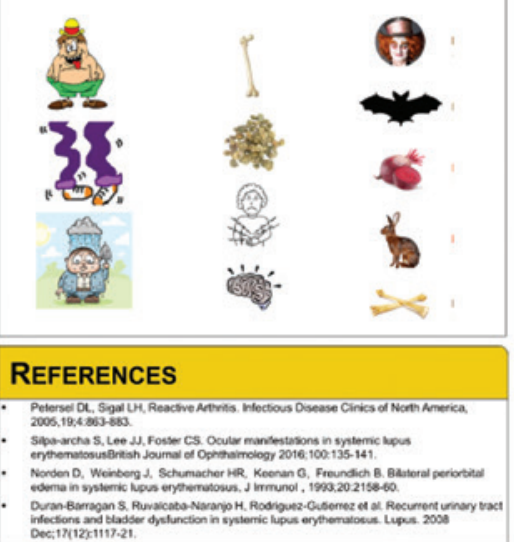

\section{Blindness Amidst Confusion?}

\section{M. Queisi, A. Bhat and K. Joshi}

Baystate Medical Center

Learning objectives: Leptomeningeal carcinomatosis should be high on the differential in any patient with active melanoma and neurological symptoms or signs of raised intracranial pressure. A complete neurological exam including fundoscopy along with a lumbar puncture are all crucial.

Case: This is a case of a 28 year old man with a history of schizoaffective disorder on antipsychotics and stage IV BRAF mutated metastatic melanoma with adrenal and nodal metastases on immunotherapy, who presented with acute onset nausea, vomiting, abdominal pain and worsening headache. His physical examination was concerning for agitation, gait instability, urinary incontinence and mydriasis. Considering recent negative CT brain, his psychiatric history and polypharmacy, he was misdiagnosed with serotonin syndrome. This was due to his escalating delirium in-house that was initially thought to be due to his underlying psychiatric disorder further delaying his work-up till his agitation was well controlled. Two days later, patient began complaining of visual loss and projectile vomiting, initial MRI brain and lumbar puncture were both negative. Given his ongoing symptoms of vision loss and high index of suspicion, a repeat lumbar puncture was performed and cytology came back positive for melanoma. Patient then underwent ventriculo-peritoneal shunt placement and initiation of intrathecal chemotherapy. After one year of failed chemotherapy treatment, family decided to initiate hospice care. 
Discussion: Leptomeningeal carcinomatosis is a rare but known complication of melanoma and should be considered in any patient with active malignancy. Symptoms for brain metastasis can easily be masked by psychiatric diseases and delirium, which can lead to diagnostic delays. A complete neurological examination including fundoscopy is pivotal in diagnosing such conditions. A repeat lumbar puncture and CSF cytology should also be performed alongside brain imaging when suspicion remains high and alternative diagnoses are ruled out.

\title{
Taking the Plunge: A Rare Case of Refractory Cerebral Venous Sinus Thrombosis
}

\author{
A. Quinn, E. Mogadam, L. Kose, M. Pirastehfar, A. Konstas and D. Banta \\ Huntington Memorial Hospital
}

Learning Objectives: Physician attitude toward risk and ambiguity may systematically affect decision-making. Aversion to risk can lead to dismissal of correct diagnoses and suboptimal treatment.

Case Information: A 30-year-old woman presented within hours of sudden-onset left hemiparesis and headache. Contrast-enhanced MRI demonstrated an occlusive thrombus extending into multiple cerebral venous sinuses. She smoked cigarettes and used a vaginal ring contraceptive. Early anticoagulation with heparin, the consensus treatment for cerebral venous sinus thrombosis (CVST), was promptly initiated. Despite early heparinization, MRI on hospital day 3 found the thrombus had propagated. Two thrombectomies were unsuccessful due to difficulty passing a catheter through apparently patent sinuses and uncontrollable bleeding with meningeal manipulation. The unusual dural character prompted secondary review of the imaging, which detected intermediate T1 signal in the occluded sinuses and mild diffuse meningeal thickening. Reported cases of CVST in patients with similar imaging abnormalities diagnosed as idiopathic hypertrophic cranial pachymeningitis (IHCP) did not improve with heparin but did respond to glucocorticoids.

CVST refractory to heparin and the dural abnormalities observed on imaging and during thrombectomy suggested underlying IHCP. Consultants recommended not to deviate from heparin therapy, arguing the risks of high-dose glucocorticoids for IHCP were unacceptable because the diagnosis was unproven. However, given the patient's continued neurologic deterioration despite heparin and signs of IHCP, pulse-dose intravenous methylprednisolone 1 gram per day for 3 days with a long taper was begun on hospital day 5. The patient's paretic extremities, unimproved thus far, had 3 out of 5 muscle strength within 24 hours of initiating glucocorticoids. Eight days later, the patient was discharged neurologically intact. At 3 months follow-up, the patient had no residual deficits.

Discussion: Risk aversion can hinder medical decision making. This case demonstrates that increased tolerance to risk and diagnostic uncertainty can profoundly benefit treatment and a patient's life.

\section{When the Script Doesn't Fit: Not All That Coughs is Pneumonia}

\author{
A. B. Rezigh and J. N. Lessing \\ University of Colorado School of Medicine, Department of Internal Medicine
}

Learning Objectives: Recognize ill-fitting illness scripts and lack of expected improvement (treatment failure) as a trigger to reconsider the diagnosis.

Case Presentation: A 27 year-old man was hospitalized for two months of cough and shortness of breath. Chest x-ray (CXR) revealed interstitial opacities. CT chest with contrast, performed given history of pulmonary embolism, was read as likely chronic pneumonia, although also mentioned was interstitial prominence and diffuse lymphadenopathy. He was treated with azithromycin and discharged. On follow-up, his symptoms only minimally had improved so doxycycline was added. Two months later he re-presented with worsening cough and dyspnea. CXR again revealed similar findings to before. He was again treated for pneumonia, this time with levofloxacin. Three days later, he represented for the fourth time, now with respiratory failure. Deeper history included learning of a mother with sarcoidosis and recent unexplained weight loss and fatigue. A CT ILD was suggestive of sarcoidosis. Lung biopsy confirmed the diagnosis of sarcoidosis.

Discussion: The estimated prevalence of sarcoidosis is 10-20 per 100,000 population. Sarcoidosis is more common in young, black adults, fitting with our patient's demographics. His initial symptoms of months of cough and dyspnea, though non-specific, are classic for this disease (1). Though his initial presentation and CXR were perhaps suggestive of community-acquired pneumonia (CAP), the months of symptoms and CT findings - interstitial opacities and lymphadenopathy - does not fit the typical CAP illness script. This, in combination with lack of improvement with appropriate CAP therapy, could have prompted further diagnostic considerations. This case reminds us that when elements do not fit the illness script and/or when treatment does not lead to expected improvement, this should be seen as an opportunity to revisit other diagnoses.

\section{Reference:}

1. Thomas KW, Hunninghake GW. Sarcoidosis. JAMA. 2003 Jun 25;289(24):3300-3. 


\section{Long-time Diagnostic Delay of a Young Woman with a Chronic Generalized Body Pain}

$\underline{\text { T. Sakai }}$

Iizuka hospital, Japan

Learning objectives: Synovitis, acne, pustulosis, hyperostosis, and osteitis (SAPHO) syndrome is a rare inflammatory condition affecting patients aged 30-50 years. Several patients present with anterior chest wall pain. Its natural history remains unclear. A previous report has described a self-limited course in a few patients; however, most patients show a relapsing-remitting or chronic indolent course after an early acute phase and often require chronic therapy. The possibility of SAPHO syndrome must be considered in young patients with chronic, predominantly precordial pain.

Case information: A 56-year-old Japanese woman with a past medical history of several episodes of peptic ulcer disease (PUD) presented with melena. She underwent emergency endoscopy and was diagnosed with PUD based on a several-month history of nonsteroidal antiinflammatory drug (NSAID) use for chronic generalized body pain of unknown etiology and was referred to our department. She reported a 20-year history of anterior chest wall pain and incision and drainage of a sterile abscess at the same site. She visited her primary physician for intermittent chest pain and received symptomatic therapy including NSAIDs; however, her condition remained undiagnosed, and her condition was attributed to malaise. She developed an occasional rash on her hands and feet, which was attributed to allergy. She was hospitalized several times for suspected NSAID-induced PUD; however, the condition necessitating NSAID use remained uninvestigated. Physical examination showed tenderness across her entire chest (predominantly peristernal). Pustules, scales, and skin exfoliation occurred on both palms and soles. Chest radiography and whole-body computed tomography on admission showed bilateral sternoclavicular erosion with sternocostal hyperostosis. She was referred to a rheumatologist and was diagnosed with SAPHO syndrome.

Discussion: Delayed diagnosis in this patient was attributable to the following factors: (1) Symptomatic treatment effectively treated her remitting-relapsing disease, and her daily activities remained relatively unaffected. (2) Her self-diagnosis masked the true diagnosis. (3) Not only her primary physician but also the doctors who treated her NSAID-induced ulcer before this admission did not investigate her thoroughly to establish a conclusive diagnosis. These led to a several-year delay in diagnosis.

\section{Acute Intermittent Porphyria: An Uncommon but Potentially Life-Threatening Cause of Abdominal Pain}

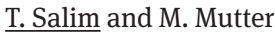

University of Pittsburgh Medical Center, Pittsburgh, PA

\section{Learning objectives:}

- $\quad$ Recognized that acute intermittent porphyria (AIP) is an under-diagnosed and treatable cause of abdominal pain, with the potential for life-threatening complications

- $\quad$ Recognize premature closure bias in diagnosing patients who present with recurrent abdominal pain

Case information: A 34-year-old female with past medical history of chronic, intermittent abdominal pain presented to the emergency department with abdominal pain, lower back pain, and dark-colored urine. She was previously seen in urgent care for these symptoms and had a negative CT abdomen/pelvis but re-presented to the ED due to worsening abdominal pain. Upon presentation, she was mildly hypertensive and tachycardic, with mild distress noted on physical exam and a sodium level of 128. Initially, a psychiatric etiology was thought to be the cause of her chronic abdominal pain, but about 22 hours after admission, the patient suffered a seizure. Repeat labs at that time resulted in a sodium level of 120 and she was then transferred to the ICU. Gastroenterology was consulted and a workup for acute intermittent porphyria (AIP) was pursued. 24-hour urine porphobilinogen returned at 25-times the upper limit of normal (100 mg/24-hours), consistent with AIP. She was subsequently started on daily hemin infusions, with resolution of her abdominal pain within 3 days.

Discussion: This patient had a history of recurrent episodes of abdominal pain, with greater than three ED visits in one week, though prior workup had not revealed a discernible cause. Diagnosis of AIP is often delayed, with an average delay in diagnosis of about 15 years. In this case, premature closure bias and zebra retreat led to a diagnostic delay until she suffered a seizure and workup was subsequently expedited. Clinicians should consider AIP in the differential diagnosis of chronic abdominal pain and pursue diagnostic testing in appropriate patients after an initial workup is unrevealing. 


\section{Shaken to the Core: Collaboration to Diagnose Convulsive Syncope}

E. Shinnar ${ }^{1}$ and R. Mehendale ${ }^{2}$

${ }^{1}$ New York Presbyterian Columbia University Medical Center

${ }^{2}$ Columbia University Medical Center - Neurological Institute of New York

\section{Learning Objectives:}

- To utilize collaboration among consultants to prevent error from triage cueing.

- $\quad$ To review non-infectious complications of HIV and an atypical presentation of convulsive syncope.

Case Information: 62 year old man with a history of HIV (last CD4 count of 500), coronary artery disease and ESRD presents with new convulsions. He experienced multiple episodes of full body shaking with loss of consciousness lasting up to 10 seconds; he would then abruptly awake without post-ictal confusion. Episodes were preceded by quick prodrome of a feeling of warmth and loss of vision. Labs revealed a hemolyzed potassium to 6.7 and a white count at baseline of 8. EKG demonstrated first degree heart block without acute ischemic changes. CT head showed a chronic left occipital infarct and chronic microvascular ischemia. He was given levetiracetam for a new seizure disorder. Concerned for meningitis, a lumbar puncture was unsuccessfully attempted. Given the atypical presentation, the neurology service consulted internal medicine to discuss other etiologies. After being placed on telemetry, the patient was noted to have episodes of complete heart block preceding the convulsions. A trans-venous pacer was urgently placed, levetiracetam was stopped and the patient had no further convulsive episodes.

Discussion: Convulsive syncope from heart block is an uncommon presentation of syncope, rarely causing full body convulsions. Convulsive syncope can be confused with seizures, which were thought to be likely in a patient with prior stroke and HIV. While neurologic infections can occur in controlled HIV, another common complication of HIV is coronary heart disease. The neurologist involved treated for seizures but was concerned for another etiology given the lack of any post-ictal state. Collaborating with the internist, the team was able to look at other causes of convulsions, ultimately arriving at the diagnosis.

\section{The Mediastinal Mass That Wasn’t}

D. Sterken, P. Sanger and D. Roseman

University of California, San Francisco

\section{Learning Objectives:}

- $\quad$ Review how framing can influence differential diagnosis, especially in communication between medicine and radiology.

- Examine how reframing can help avoid biases such as confirmation and anchoring.

- $\quad$ Reinforce the importance of obtaining history directly from the patient rather than secondary sources.

Case Information: A 60 year-old woman with HTN and HLD presented to our hospital for biopsy of a mediastinal mass discovered at an outside hospital. She had presented to that hospital a week earlier with headache, for which a head CT was obtained. This and subsequent imaging revealed a left neck "mass-like lesion" and a heterogeneous mass in the superior mediastinum encircling the esophagus, as well as mixed lytic and sclerotic osseous lesions along with a diffusely "mottled appearance" of osseous structures. Other notable findings included hypercalcemia. In discussion with our radiologists, her imaging did not fit any of the classical etiologies of mediastinal masses. The patient had told us, however, that her first symptom was actually left neck swelling and pain. She had then massaged her neck, after which the swelling abruptly decreased in size and she developed bruising over her anterior neck. We thus reframed her case as a neck mass and were thereby able to reach a final diagnosis of parathyroid carcinoma with osteitis fibrosa cystica from hyperparathyroidism. Her "mediastinal mass" was in retrospect thought not to be a mass at all, but rather blood that had descended into the mediastinum from her neck mass causing chemical mediastinitis - which has been previously described - after hemorrhage due to mechanical disruption.

Discussion: When a patient's presentation does not fit any of the illness scripts in our differential diagnosis, we should consider reframing our problem representation in order to find a more appropriate diagnostic schema for the case, preferably using primary information from the patient.

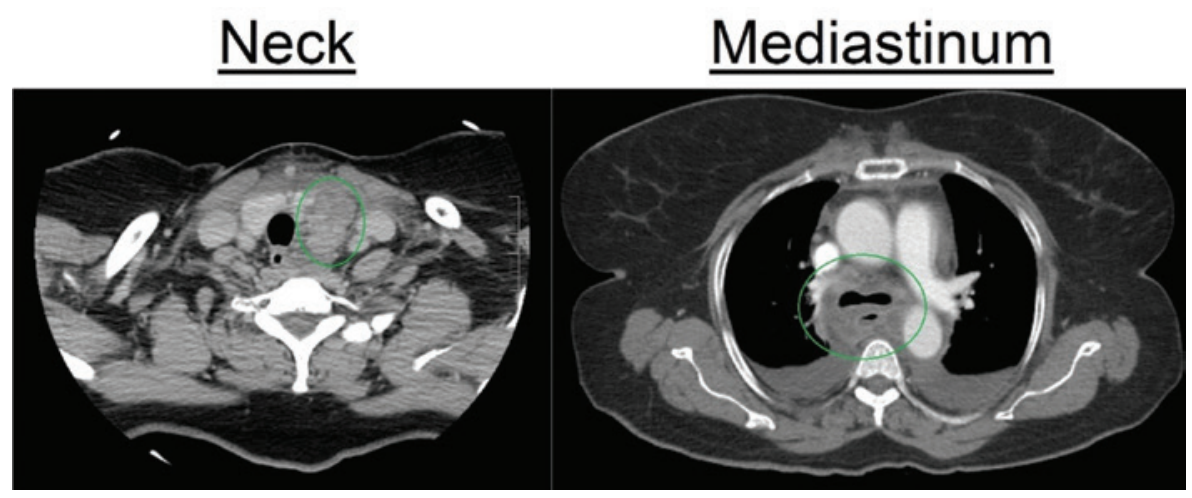




\title{
Multiple Myeloma with Delayed Diagnosis
}

\author{
D. Sugawara, S. Tomiyama, H. Imura
}

Department of General Internal Medicine, Izuka Hospital, Fukuoka-ken, Japan

Learning objectives: When we suspect acute osteomyelitis with few signs of infection, it is important to consider other differential diagnosis such as multiple myeloma.

Case information: This is 85 -year-old male who complained of a month history of numbness of the left lip. A CT scan showed a $19 \times 9$ mm irregular bone decalcification below the lower root of the lower left 7 th and 8th tooth, and suspected acute osteomyelitis. There were no signs of infection such as fever, swelling, or pain. We diagnosed osteomyelitis and started antibiotics but there was no improvement. 2 weeks later, L/D showed CRP $22.4 \mathrm{mg} / \mathrm{dl}$ and continued to treat with antibiotics on administration. His general condition was improved and fell to CRP $1.9 \mathrm{mg} / \mathrm{dl}$ on day 14 of treatment and extract the teeth. 2 months later, L/D showed CRP $29.8 \mathrm{mg} / \mathrm{dl}$ and he was re-hospitalized for acute osteomyelitis relapse. There was granulate at left lower gum, we obtained biopsy and suspected plasmacytoma. Bone marrow biopsy showed $80 \%$ of nucleated cells which were occupied by plasma cells. We diagnosed multiple myeloma and started Ld therapy. However, 2months later, he suffered from MSSA bacteremia and he died.

Discussion: We experienced the case which we first treated as osteomyelitis but 3 months later diagnosed as multiple myeloma. There were no signs of infection but we ignored it. Premature closure due to anchoring bias caused a delay in diagnosis. When we see bone lesion, it is important to consider multiple myeloma. The numbness of the left lip was considered Numb chin syndrome. Numb chin syndrome is caused by damage to the mental nerve and inferior alveolar nerve and is known to present as a symptom of multiple myeloma so we should require attention.

\section{Misdiagnosis in a Patient Suspected Urinary Tract Infection}

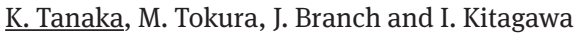

Shonan Kamakura General Hospital

Learning objectives: Comparing patient records, such as computed tomography (CT) scan images, before and after disease progression can aid in an accurate diagnosis of the presenting condition. Additionally, contrast magnetic resonance imaging (MRI) should be used for the diagnosis of metastatic brain tumor instead of CT.

Case information: A 76-year-old man with a history of schizophrenia and bladder catheterization for neurogenic bladder dysfunction, related to diabetic neuropathy, presented with a one-week history of mental status change. His drug regimen was reduced prior to presentation at our hospital because of suspected over-sedation, but his mental status did not improve. His inflammatory markers were slightly elevated, and he exhibited bacteriuria. A head to pelvis CT scan incidentally revealed lung cancer with adrenal metastases. However, these findings did not explain the mental status changes. He was commenced on antibiotics, but no improvement in mental status was noted. Although, his speech had normal fluency and volume before symptom onset, he became dysphasic beginning one week prior to presentation at our hospital. On comparing his current head CT with one taken in the past, due to a head injury, we noticed ventricle enlargement. This led us to suspect that the lung and adrenal malignancy had metastasized to his brain. We performed a head MRI (with and without contrast), which confirmed metastatic disease.

Discussion: This case was misdiagnosed due to availability bias. The patient's schizophrenia also contributed to the misdiagnosis. This case highlights the importance of comparing current images with those obtained prior to symptom exacerbation. Plain CT scan may be insufficient for the diagnosing of brain metastases. When suspected, MRI scanning may be more useful in such circumstances.

\section{The Importance of Cardiac Screening in Patients with Melanoma - A Case Report}

$\underline{\text { A. Tandon }}^{1}$, A. Mehlman ${ }^{1}$, C. J. Bitetzakis ${ }^{1}$, K. Kongpakpaisarn ${ }^{1}$ and D. Whitaker ${ }^{2}$

${ }^{1}$ University of South Florida, Department of Internal Medicine, Tampa, FL

${ }^{2} J a m e s$ A Haley Veterans Hospital, Department of Cardiology, Tampa, FL

Learning Objective: Importance of cardiac screening in patient with melanoma in setting of non-specific symptoms

Introduction: Metastatic melanoma accounts for $4.4 \%$ of cardiac metastases (1). Pericardial effusions associated with metastases to the heart are a frequent finding in malignant disease (2); However, cardiac tamponade is an uncommon presentation of malignancy (2). We present a case of metastatic melanoma presenting with vague features leading to delay in diagnosis of tamponade.

Case: A 65-year-old male with remote history of resected localized melanoma presented with a two-week history of dysphagia and mild dyspnea. He underwent a barium swallow revealing distal esophageal narrowing with concern for stricture or mass. The final pathology from the esophageal gastroduodenoscopy (EGD) showed no concern for malignancy. His symptoms were ongoing and a computed tomography (CT) scan of the chest two weeks later, revealed a mass compressing the left atrium and pulmonary vessels. Biopsy obtained was consistent with metastatic melanoma. Over the proceeding days the patient went on to have progressive dyspnea. A transthoracic echocardiogram (TTE) 
showed large pericardial effusion with right atrium collapse and a mass compressing the left atrium (Figure 1) consistent with tamponade physiology. He underwent emergent subxiphoid pericardial window with pericardial drain placement.

Discussion: The propensity of melanoma to metastasize to the heart is well documented. Unfortunately, diagnosis is often late due to a lack of specific cardiac symptoms even in advanced disease, as seen in our patient (3). This leads to non-specific testing and delay in the diagnosis (3). Currently, there are no guidelines for cardiac screening in patients with melanoma (3). Our patient was in monitored settings, enabling us to act before there was a progression to cardiogenic shock. Therefore, this case highlights the importance for having a low threshold to evaluate patients with a new or remote diagnosis of melanoma presenting with cardiac or vague otherwise unexplained symptoms.

\section{References:}

1. Aerts, B. R., et al. (2014). “Cardiac metastasis of malignant melanoma: a case report.” Netherlands Heart Journal 22(1): 39-41.

2. Muir, K. W. and J. C. Rodger (1994). "Cardiac tamponade as the initial presentation of malignancy: is it as rare as previously supposed?" Postgraduate Medical Journal 70(828): 703-707.

3. Ramchand, J., et al. (2016). “Cardiac metastatic melanoma.” BMJ Case Reports 2016.

Figure 1 Transthoracic Echocardiogram demonstrates a large mass compressing the left atrium, with associated pericardial effusion compressing the right atrium.

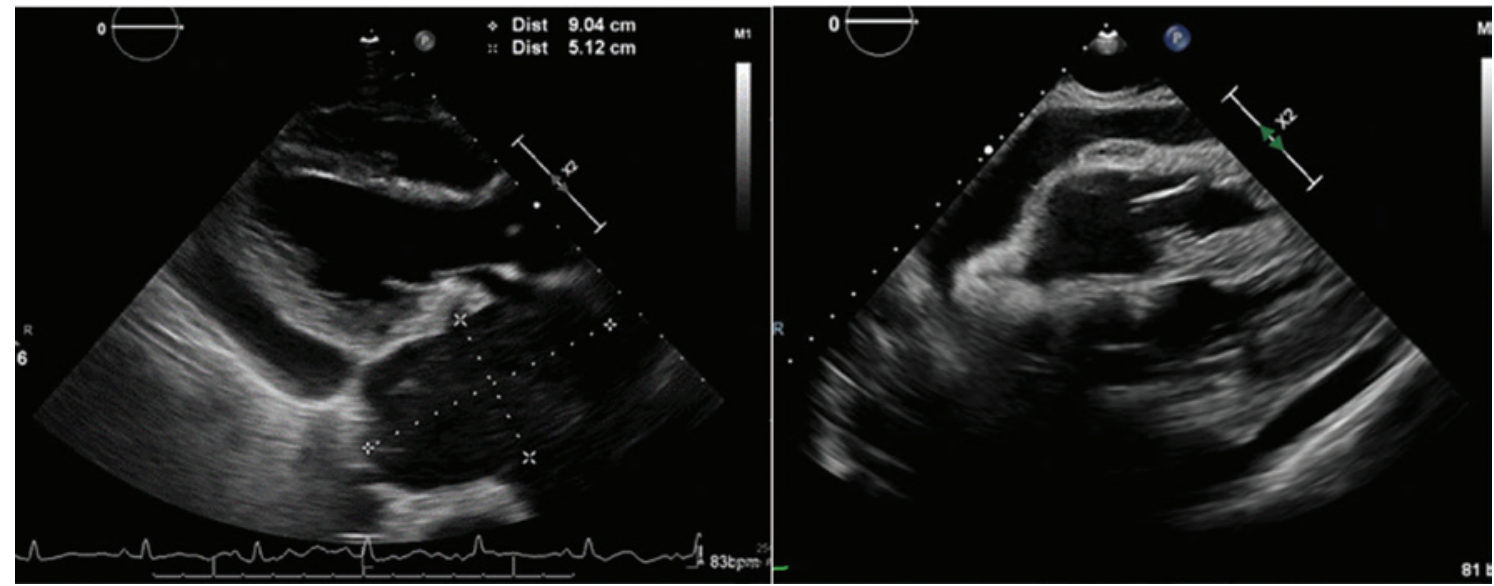

\section{Prepatellar Septic Bursitis (Housemaid’s knee) Complicated by Lymphangitis}

T. Watari

Shimane University Hospital

An 83-year-old Japanese Tatami craftsman with underlying diabetes mellites came to our emergency room complaining of severe pain and feeling of warmth in his right knee with mild chills. Three weeks prior to admission, he had noticed a tiny piece of wood lodged in his right knee. Although at the first visit a marked swelling, tenderness, and redness was observed on the anterior part of his knee, he was diagnosed with pseudogout and sent home without knee aspiration. The next day he re-visited the ER complaining of severe pain. In addition to inflammation in the front right knee, a limited range of motion, and redness extending from the right buttock to the medial thigh indicative of lymphangitis was also observed. Magnetic Resonance Imaging of the knee showed no significant abnormalities in the joint space; however, there was fluid accumulation in the prepatellar bursa. Staphylococcus aureus was detected in the synovial fluid culture, confirming the diagnosis of prepatellar septic bursitis (Housemaid's knee). He was hospitalized immediately and treated by fluid aspiration and administering intravenous first-generation cephem antibiotics which led to recovery without any sequalae.

The prepatellar bursitis was caused by skin blemishes, and it is common amongst housemaids, carpet layers, clergy, gardeners, who frequently use the kneeling position. The main symptoms typically include pain, peri-bursal erythema, and warmth. Fever is seen only in 40-44\% of the cases. For a correct diagnosis, gram stain and culture of the aspirated bursal synovial fluid must be performed if septic bursitis is suspected since it is difficult to differentiate between an infectious and non-infectious disease without above findings. Staphylococcus aureus is the main causative organism in more than $80 \%$ of culture-proven septic bursitis cases. In conclusion, if lymphangitis is suspected, bacterial infection should be considered from the distal part. 


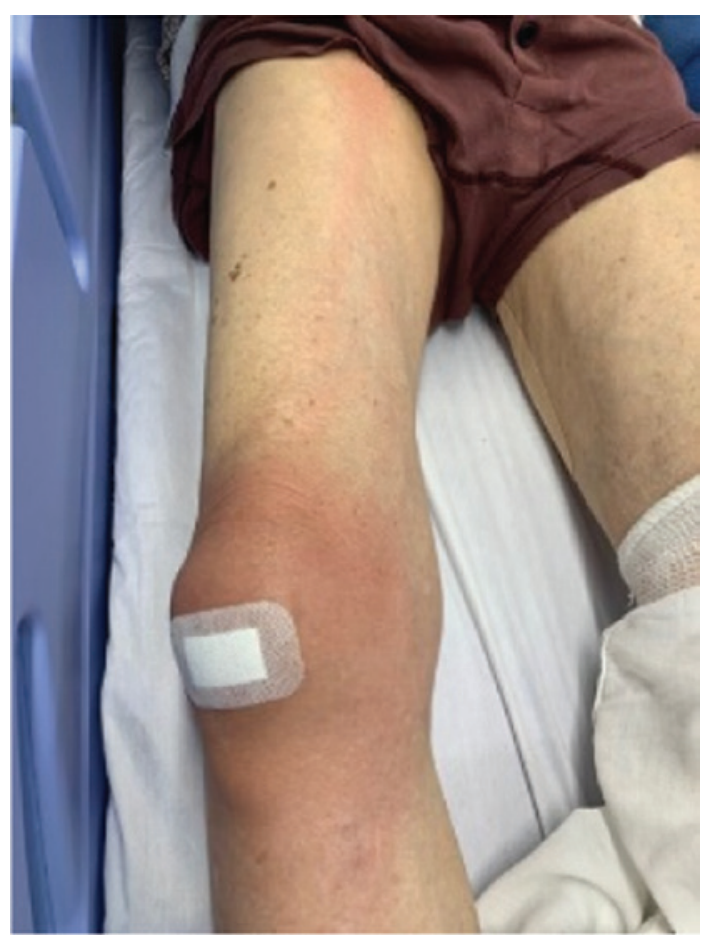

\title{
Obesity Bias Causing Delay in Diagnosis of Eosinophilic Pneumonia
}

\author{
C. Wiswall and H. Hoar \\ University of Massachusetts Medical School, Baystate
}

\section{Learning objectives:}

- Acknowledge obesity bias as a potential cause of diagnostic delay

- Discuss strategies to mitigate delays caused by obesity bias

Case Information: NM is a 17 year old with mild intermittent asthma and super morbid obesity (BMI 54) who presented to the hospital with dyspnea and cough for several months. CT angiogram performed on presentation showed worsening of a pre-existing infiltrate. She was admitted to the hospital with multiple specialists consulted to elucidate a diagnosis. Rheumatology consulted on day three of hospitalization recommended tissue biopsy over bronchoscopy as vascular tissue samples were imperative to help rule out vasculitis and other causes; infectious disease consultant concurred. Pediatric surgery recommended against VATs biopsy in favor of bronchoscopy, citing heightened morbidity from surgical procedure. Bronchoscopy performed on day eight of hospitalization was non-diagnostic. She clinically worsened while awaiting a diagnosis, transferred to PICU for respiratory failure. Thirteen days into her hospital course patient underwent tissue biopsy via VATs, which secured the diagnosis of eosinophilic pneumonia.

Discussion: Obesity bias is a form of implicit bias that can result in a variety of diagnostic errors. In this case, there was diagnostic uncertainty in a patient with CT findings consistent with necrotizing pneumonia - a differential that included infection, vasculitis, granulomatous disease, and connective tissue disease. Primary and consultant teams agreed that tissue biopsy was necessary to secure diagnosis. Surgical consultant teams deferred VATs biopsy on several occasions, citing risks of the procedure. Almost two weeks into her hospital course, tissue biopsy was performed which secured the diagnosis and directed the treatment strategy. We postulate that obesity bias played a large role in this diagnostic delay, given patient's super morbid BMI and multiple references to increased post-procedural morbidity. Diagnostic delay from obesity bias can be mitigated if consultants explicitly state the risks of procedures due to obesity, so that care providers, families, and patients can make fully informed decisions on risk-benefit. 


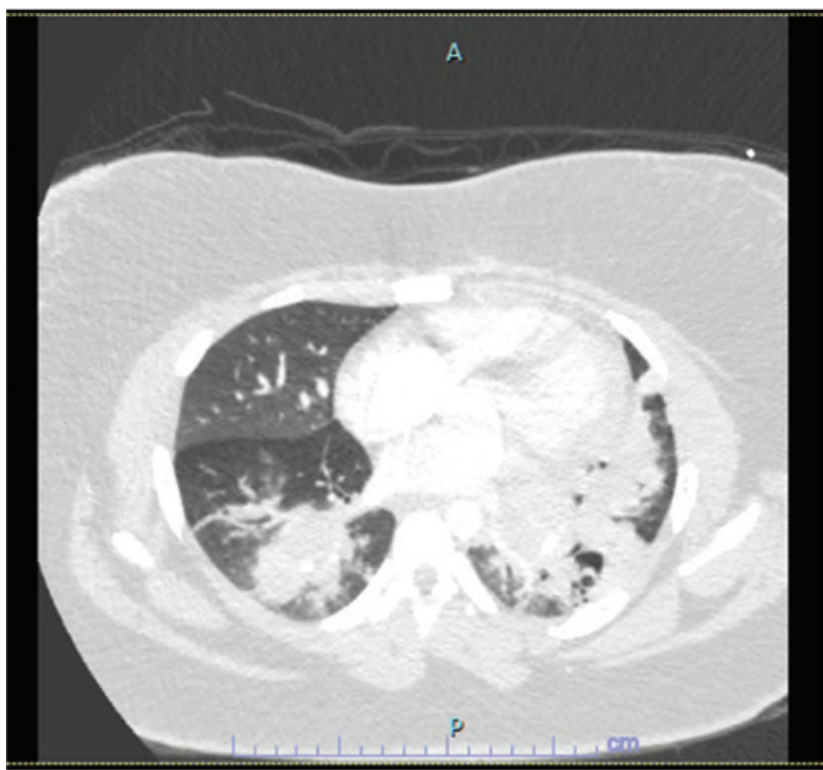

\section{Education}

\section{Designing a Computerized Sepsis Training Program}

\section{Engel, M. C. Wright and L. Vander Hart}

Trinity Health

Purpose/problem: Sepsis is a key driver in health care costs, is a leading cause of hospital readmission, and impacts more than 1 million patients seen in hospitals each year. While the diagnostic burden of sepsis includes early recognition by the public, first responders, and physicians, in-hospital recognition and appropriate treatment of septic patients continues to be a struggle. Educating frontline health care workers is a challenge that is complicated by lack of time, an evolving workforce, and ever-changing demands.

Description of program: A computer-based self-administered training module was developed around a micro learning platform. Using expert guidance and a review of the literature, we developed an interactive, case-based training program for frontline healthcare workers in our organization. Modules incorporated gamification to aide in engagement and feedback following each module that summarized key learning points.

In general, training modules were focused on making appropriate triage decisions for fictitious patients, given a set of vital signs and physical exam findings. In initial modules, learners would engage in sorting signs and symptoms by whether they were concerning for sepsis. Later modules had learners prioritize patients by their disease severity.

Outcomes: Vignettes were generated and are being presented to frontline providers in our institution. The final learning platform (figure 1) allows for the addition of more modules or modification of existing cases by educators as needed. Learner outcomes are forthcoming.

Discussion: Microlearning has a potential to engage health providers to educate and reinforce knowledge and desired behaviors in patients where sepsis is suspected.

Lessons learned: De novo development of a learning platform requires engagement with stakeholders at all levels. In delivering an education tool based around microlearning to frontline health care workers, attention must be made to tailor the learning to their level of understanding in an effort to both reinforce their existing knowledge base while also building new skills for recognizing patients with and at-risk for sepsis. 


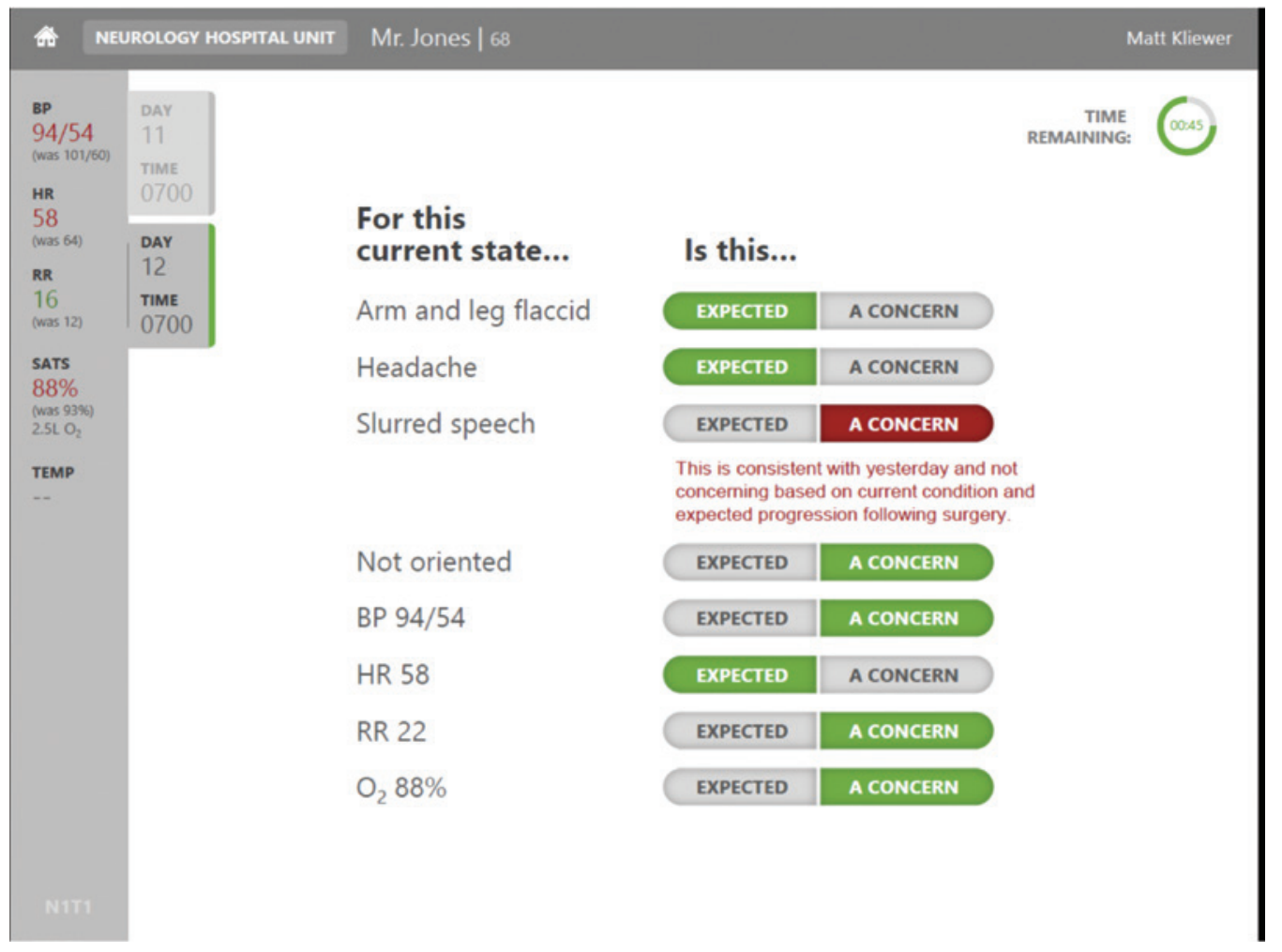

\title{
Diagnostic Excellence: Getting it Right at the Right Time - Key Messages from Internal Experts
}

\author{
$\underline{\text { R. Loo }}^{1}$, M. Kanter ${ }^{2}$, N. Gin ${ }^{1}$, M. Arteaga-Garavito ${ }^{1}$ and M. Hugh ${ }^{1}$ \\ ${ }^{1}$ Southern California Permanente Medical Group \\ ${ }^{2}$ Kaiser Permanente School of Medicine
}

Purpose/Problem: Diagnosis is among the most critical and complex challenges physicians face every day, and diagnostic errors are a significant patient safety concern. Misdiagnosis not only takes a toll on patients and their families, but also on health care practitioners, and their colleagues. Raising awareness of the breadth of the problem, explaining common ways errors are made, and reminding practitioners of solutions and resources to mitigate errors is a one step towards diagnostic excellence.

Description of program, assessment, or study: An educational video for clinicians was successfully developed to raise awareness on the factors that lead to diagnostic errors and how to achieve diagnostic excellence. The research based content was developed with input from internal subject matter experts and leaders, including patient advisors, to convey key messages and strategies that include: explanation on how and why diagnostic errors are made, how to avoid diagnostic errors and improve decision-making by engaging and collaborating with patients and teammates, documenting differential diagnoses and using available resources and systems that support physicians and patients in assuring diagnoses are timely, accurate and communicated efficiently.

The video also addresses the importance of provider wellness by creating a culture of learning and psychological safety. A healthy learning culture enhances quality, safety and clinical confidence, prevents burn-out, reduces bias, and leads to a streamlined diagnosis and treatment pathway.

The value of having leaders and patient partners on the screen allows for internal experts to share their knowledge and expertise and identifies leaders as resources and advocates for diagnostic excellence.

Outcomes/Discussion/Significance of findings: The video is a requirement for physicians, and performance is monitored through knowledge checks. Viewers' feedback is gathered for continuous improvement. The socialization of the tool is done through existing educational structures aligned with internal incentives. In addition, physicians each Continuing Medical Education (CME) credits upon completion. The material presented is beneficial to a diversity of healthcare providers, and the scope can be expanded to incorporate viewers from other entities.

This enduring material is part of a larger strategy to achieve diagnostic excellence. Other initiatives that complement this work include guides to enhance patients-clinician partnering, online practice of diagnostic skills, and diagnostic clinical reasoning support systems. All initiatives are developed in synchrony to align messaging, ensure effectiveness and cost-efficiency.

A similar abstract was accepted for the 2019 IHI National Forum on Quality Improvement in Health Care 


\section{The Clinical Problem Solvers: An Internal Medicine Podcast Dedicated to Teaching Approaches to Common Medical Problems}

D. Minter ${ }^{1}$, S. Shekarchian' ${ }^{1}$ A. Derakhshan ${ }^{2}$, R. Manesh ${ }^{2}$ and R. Geha ${ }^{1}$

${ }^{1}$ University of San Francisco School of Medicine.

${ }_{2}^{2}$ Johns Hopkins University School of Medicine.

Purpose: The rise of social media has transformed the way we communicate. A systematic review (PMID 23619071) concluded that interventions using social media tools were associated with improved knowledge, attitudes, and skills. These platforms, particularly Twitter and podcasts, have become increasingly popular in the medical community.

Clinical reasoning is fundamental to the practice of medicine, but this complex process is rarely modeled as teachable habits. Rather, it is often developed through trial and error. The landmark Improving Diagnosis in Health Care report identified the lack of focus on developing clinical reasoning as a "major gap in education within all health care professions" which then contributes to diagnostic error.

The gap identified in clinical reasoning education and the far-reaching power of social media inspired us to create The Clinical Problem Solvers (CPSolvers) - an internal medicine podcast dedicated to diagnostic reasoning through a case-based, iterative process.

Description: The CPSolvers is a free weekly podcast, featuring two types of episodes:

1. Schema based: focus on teaching a systematic approach to commonly encountered problems through patient cases (e.g., abdominal pain) and

2. Unknown case: a blinded discussant is presented a clinical case and shares her clinical reasoning extemporaneously.

3. Our website (clinicalproblemsolving.com) has downloadable versions of discussed schemas and videos which walk the viewer through the majority of our schemas.

Outcomes: Since the launch of CPSolvers in December 2018, we have produced 11 schema based and 27 unknown episodes. Eleven of these unknown episodes were done in collaboration with the Human Diagnosis Project. CPSolvers attracts 5,500 listeners per episode in 137 countries. Our website has over 100,000 visits to date and our products have been used as curriculum at multiple institutions worldwide.

Discussion/Significance of findings: We highlight the worldwide reachability of a podcast dedicated to diagnostic reasoning.

\section{Cross-Cultural Experiences Teaching Clinical Reasoning in Turkey}

\section{Schaye, R. Rabinowitz and N. Bertelsen}

NYU School of Medicine

Problem: Much of the medical education literature on clinical reasoning (CR) comes from primarily English-speaking countries. It is less clear how these educational interventions translate to environments with non-native English speakers, and in clinical systems where there is higher patient volumes.

Purpose: To implement a CR session for 6th year medical students at a university hospital in Istanbul, Turkey.

Description of Program: In November, 2018 we conducted a 3-hour session on CR at Koc University in Istanbul developed from a curriculum at our home institution in the United States.

In an interactive case-based didactic, students were introduced to the CR concepts of problem representation (PR), illness scripts, and diagnostic time outs. Students then applied these concepts to a case during facilitated small group breakouts.

Outcomes

Twenty-four 6th year medical students participated. Retrospective pre-post self-assessments of confidence in and likelihood of using CR concepts on an eight-point Likert scale were completed, and qualitative data on use in clinical practice were obtained. Results were analyzed using a paired t-test. After participating in this session, students were more confident in and more likely to use all domains of the CR framework taught with large effect sizes in eight of ten domains (Figure 1). A common theme in the qualitative data was that the use of PR would be helpful to communicate about patients efficiently in the busy clinical environment.

Discussion: Teaching CR skills translated well to an international setting. Critical to the session's success was demonstrating relevance to the local institution's clinical environment, in particular taking into account the higher patient volume experienced by providers, and changing the attitude that better decision-making does not necessarily take longer. The impact of culture and environment was evidenced by what the students found to be most effective-use of PR to communicate efficiently about patients in a busy clinical environment-which is different from our experiences teaching this in the US where learners often highlight the utility of the diagnostic time out.

Significance of Findings

Our findings speak to the need to contextualize CR concepts within the local learning culture to define relevance and meet the learners' needs. 
Figure 1. Retrospective Pre-Post on Confidence in Ability to Apply and Likelihood of Using Clinical Reasoning Skills

\title{
Confidence in Ability to Apply and Likelihood of Using \\ Clinical Reasoning Concepts Taught
}

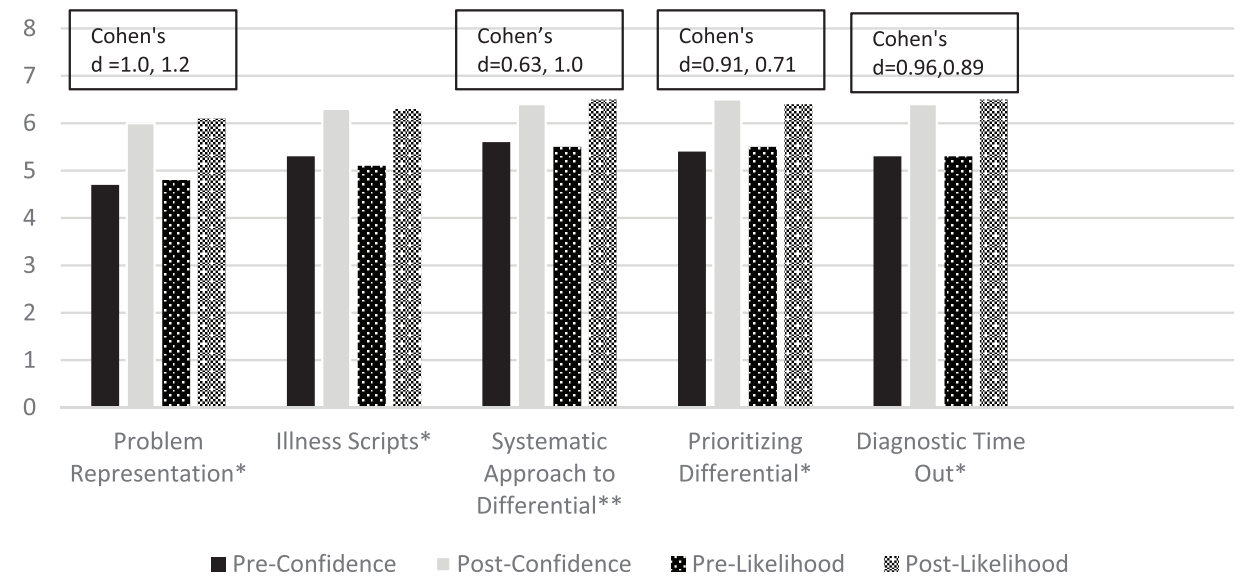

${ }^{*} p<0.001,{ }^{* *} p=0.01$

\section{Incorporating Diagnostic Reasoning Education into Nurse Practitioner Education Programs: Challenges and Opportunities}

\author{
S. K. Smith \\ University of Minnesota, School of Nursing
}

Purpose: This presentation will discuss challenges and opportunities of introducing diagnostic reasoning (DR) education into Nurse Practitioner (NP) education programs. The presentation will additionally provide examples from an evolving longitudinal DR curriculum in an Adult-Gerontology Nurse Practitioner (AGNP) program.

Description of the program: While clinical reasoning in general and some components of diagnostic reasoning have traditionally been included in NP education programs, explicit and intentional teaching of diagnostic reasoning as a specialized competency has typically not been included as part of NP education. To address the need for better and more intentional development of NP students' DR skills, a longitudinal DR curriculum is under development at this upper Midwest AGNP Doctor of Nursing Practice program. Rationale for adding this content as well as strategies for accomplishing program adjustments will be shared.

Outcomes: A formal longitudinal curriculum on diagnostic reasoning for NP education has been developed, integrated with the existing AGNP curriculum, and developmentally scaffolding DR content and skill development. The development of strategies to assess students' DR competencies is currently in progress.

Discussion: While research on outcomes of care provided by NPs is very favorable (Swan, Ferguson, Chang, Larson, \& Smaldone, 2015), little research has focused on NP competencies in DR. Clinical reasoning at the advanced practice level, and DR in particular, is a key cognitive competency that must be mastered for safe and effective independent practice as an NP. This is a challenging transition in clinical thinking for the baccalaureate- or masters-prepared RN, and is key to the subsequent development of appropriate plans for patient treatment and management. Arguably, the strength of nurse practitioners' diagnostic reasoning skills is directly related to the quality of health outcomes achieved through nurse practitioner-provided healthcare. Currently, little intentional curriculum or teaching/learning activity on diagnostic reasoning is included in nurse practitioner programs. In fact, while medical education is developing robust diagnostic reasoning curricula, a recent literature search identified no such publications from scholars or teachers focused on nurse practitioner education.

Significance: The development of intentional, well-designed, and effective curriculum and teaching/learning activities for nurse practitioner education is a significant need and was the purpose of this initial pilot project. To the extent that error can be reduced among NPs through improved education in DR, it is reasonable to assume that quality outcomes and the already positive impact of NP care may be further improved through curriculum development in this area. 


\title{
Family Feud Meets Diagnosis: Using Gamification to Teach Clinical Reasoning to Pre-clerkship Medical Students
}

\author{
T. P. Wijesekera
}

Yale University School of Medicine

Purpose/Problem: Prioritizing a differential diagnosis- a key step in clinical reasoning- is a challenging skill to develop, ranking last in selfreported comfort on a survey of Yale School of Medicine (YSM) rising clerkship students.

\section{Description}

- $\quad$ Setting: The pilot workshop took place on January 2019 for 70 minutes in a YSM classroom.

- Participants: There were a total of 120 students and 10 faculty. Each session had eight students and two faculty (one attending and one 4th-year student).

- Preparation: Clerkship directors provided a list of the 10 most important chief concerns (e.g., syncope) for their respective rotations. Case scenarios were created for six chief concerns. For each case, the faculty provided their own differential diagnoses, from which an answer key with point values was created. These values were uploaded into a PowerPoint formatted like the game show "Family Feud".

Implementation: For each round, both teams read each clinical scenario, took turns sharing their differential, explained their reasoning, and received points based on how high each diagnosis was on the faculty differential. Faculty ended each round with a 5-minute discussion modeling their own clinical reasoning on the case. The team who had the most points at the end of the six rounds won.

Outcomes: On a scale of 1-5 (i.e., poor, fair, good, very good, and excellent), students rated the workshop a 4.68, significantly higher than any of the eight clinical reasoning workshops over the past four years (range 3.27-4.25). One representative student comment was: "Family Feud was INCREDIBLE! I wish we used this more during the preclinical curriculum... Creating comprehensive differentials is something I think we are all struggling with, and both the format and the clinical guidance from the student and attending present were so helpful and engaging. It was a really fun environment to make mistakes and learn.”

Discussion: Our "Family Feud" game showed significant promise as an educational tool for teaching clinical reasoning by employing gamification to engage and motivate students while giving them the opportunity to learn through targeted pre-reading, discussion with colleagues, and faculty role modeling.

Significance: This innovation helped us understand how gamification could be used to engage learners and teach clinical reasoning. As part of the SIDM Dx Learning Collaborative, we have encouraged this "Family Feud" model into various pre-clerkship and clerkship curricula. We hope this innovation can encourage innovative approaches for teaching clinical reasoning at other institutions.

\section{Practice Improvement}

\section{Patients as Research Partners in Improving Diagnosis}

\author{
$\underline{\text { M. Arteaga-Garavito }}^{1}$, K. Litman ${ }^{1}$, S. Becken ${ }^{2}$ and R. McCauley ${ }^{1}$ \\ ${ }^{1}$ Southern California Permanente Medical Group \\ ${ }^{2}$ Burbank, CA, USA
}

Problem Statement: Diagnostic errors are often due to avoidable communication failures between patients and clinicians; however diagnostic error reduction traditionally focuses on the clinician perspective alone. Engaging patients in the development of interventions to reduce diagnostic errors, from the conceptualization and planning phases to the implementation of these projects, leads to more relevant, reliable and patient-centered improvements.

Description of intervention/program: In 2018, Southern California Permanente Medical Group (SCPMG) successfully partnered with patients to develop visit preparation tools to enhance communication between patients and their clinician. This valuable partnership with Patient Partners led to the next phase of the project: an evaluation of the visit preparation tools in actual patient visits to assess their ability to improve communication and the diagnostic process. SCPMG's partnership with Patient Partners resulted in the following outcomes: 1) development of research methods to evaluate the success of the visit preparation tool intervention; 2) inclusion of valuable patient perspectives that otherwise would not have informed this work, including patient perspectives on data collection methods, study settings, visit preparation materials, recruitment of study participants and data interpretation; and 3) increased patient empowerment by building opportunities for Patient Partners to raise concerns, provide feedback, and participate meaningfully in the evaluation and research process. Patient Partners' participation was made possible in part through a Patient-Centered Outcomes Research Institute (PCORI) grant, which trained patient partners in research methods, as well as on our organization's Patient Advisory Council programs. By collaborating with Patient Partners on the team, more patient-centered outcomes were evident in all study stages. Research for patients, done with patients, assured everyone's perspective was considered - the patients, their caregivers and clinicians. 
Findings: Patient Partners' involvement in the design and evaluation of the visit preparation intervention allowed evaluation of whether the following outcomes were achieved: utilization of visit preparation tools, willingness of patients and providers to use a communication tool, and overall perception of how these tools impacted effective office practice. This evidence identifies where and how visit preparation tools can be effectively used to improve communication and reduce the potential for diagnostic errors or delay.

Lessons learned: Engaging with patients in every stage of a research project is extremely valuable in making the intervention and evaluation processes relevant, reliable, and patient-centered. Similar successful patient partnerships are possible in most diagnostic improvement work and should be considered an essential component in designing effective research studies.

\section{Making Diagnostic Error (Reporting) Easy - How About an App?}

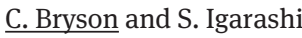

Baystate Medical Center, Springfield, MA

Statement of the Problem: Diagnostic error is a common occurrence in the hospital. While errors resulting in harm are often captured in safety reporting systems, "near miss" events are rarely reported. We hypothesized that if physicians had access to a quick and easy reporting system, we could capture medical errors that did not rise to the level of harm but that could provide useful epidemiologic data as well as educational and quality improvement opportunities.

Description of the intervention: We collaborated with IT to create a phone app. The app takes less than 5 minutes to report a case and is peer review protected. To begin a submission the provider enters an account record number or scans a patient QR code. The submission consists of an event date, attending service, admitting diagnosis (defaulting to ICD 10), final diagnosis if different than admitting diagnosis, and a brief event summary. The information is recorded in a web-based data portal, accessible to specified reviewers for analysis. The cases are categorized by the reviewers as diagnostic error, preventative error, treatment error, communication error or null. Opportunities for feedback, conference discussion, and systems improvement were pursued.

Findings to Date: 54 submissions have been received, with 51 analyses completed. 36 diagnostic, 5 preventative, 8 treatment and 1 communication error were identified, with 1 declared null. 23 providers received personal feedback. 13 cases were sent to formal peer review committees in medicine and other specialties. 3 cases were identified for potential system improvement. One system-wide root cause analysis was performed. A number of cases have been queued for morbidity and mortality conferences.

Lessons Learned: This intervention shows promise. Barriers encountered included getting providers to download the app, which had a number of steps involved. Once the app was in place, getting providers to both identify errors and take time to input them has required cultural change to overcome resistance to reporting colleague's possible errors. Multiple educational interventions were performed to ensure that providers were aware this is an educational and improvement tool rather than a component of individual performance review. Once reports were submitted, case review was time consuming and more support will be needed. Lastly, a quick feedback loop is needed to ensure providers know their cases have been received and are being addressed. Ideally an IT solution within the app would likely lead to greater engagement.

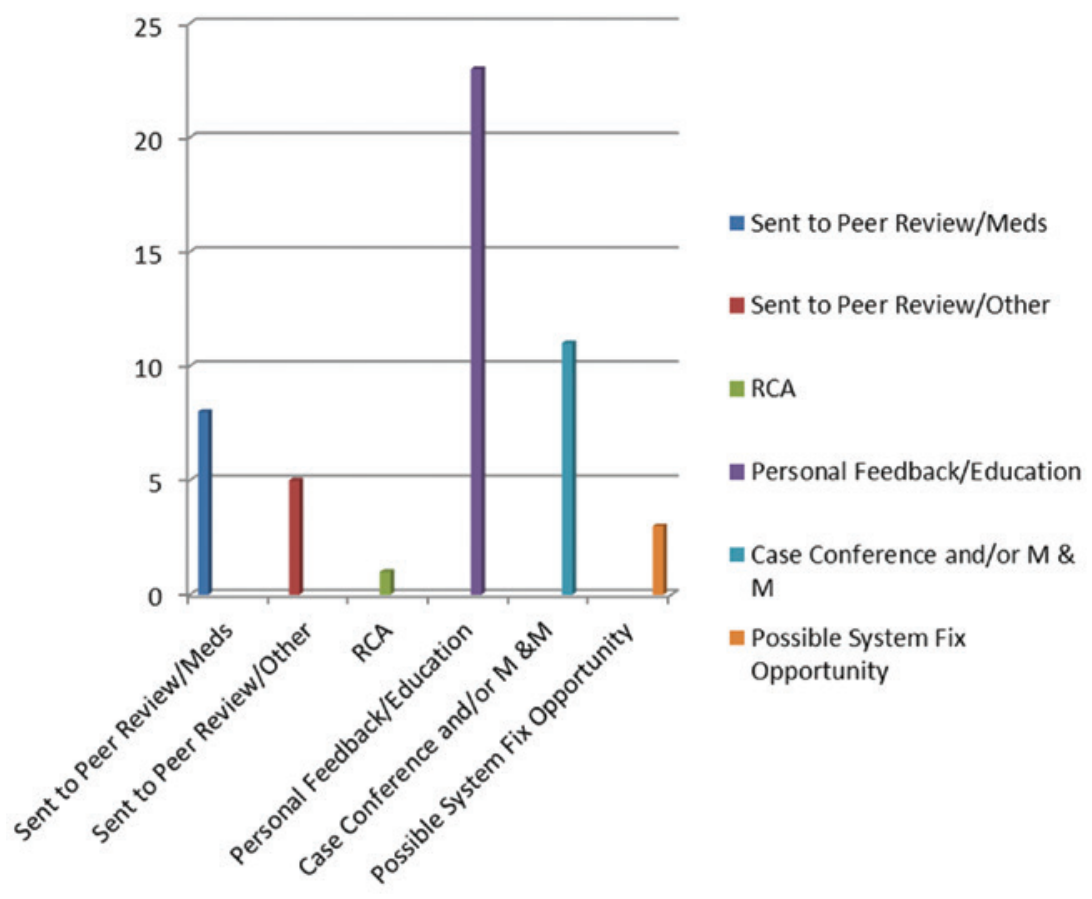




\section{Development of a “Learning List” to Provide Systematic Feedback of Diagnostic Outcomes within a Commercial Electronic Health Record}

$\underline{\text { R. El-Kareh }}{ }^{1}$, A. Kemp ${ }^{1}$ and S. Hurst ${ }^{3}$

${ }^{1}$ Department of Biomedical Informatics, UC San Diego Health, La Jolla, CA

${ }^{2}$ Information Services, UC San Diego Health, La Jolla, CA;

${ }^{3}$ Department of Family Medicine and Public Health, UC San Diego, La Jolla, CA

Statement of Problem: Feedback of diagnostic patient outcomes is crucial for clinicians to effectively calibrate their diagnostic decision making. However, fragmented care models lead to multiple handoffs of care and significantly hinder this feedback. We sought to develop a system integrated into our electronic health record (EHR) to provide consistent feedback of diagnostic patient outcomes through an automated and individualized "Learning List."

Description of Intervention: We are taking a multi-level approach to understand the various perspectives of stakeholders involved in the "Learning List" that highlights events across transitions of care. We conducted interviews with front-line clinicians and patient safety leaders within our organization to guide iterative changes to the design of the system.

We implemented our prototype Learning List system using Crystal Reports (SAP SE, Walldorf, Germany) connected to the relational database for our commercial EHR (Epic, Verona, WI). The system identifies patient handoffs and a set of post-handoff events that were previously identified to be potentially indicative of a diagnostic delay or error. This set includes events such as death, code blue, escalation of care, representation to the emergency department, early transfer between medical and surgical services and others. The system is available to all providers on eligible service teams upon electronic request and is generated daily. Each provider's Learning List contains all patients handed off by that provider during a set look-back timeframe.

Findings to Date: Through interviews with physicians, we identified data elements of highest value to remind them of the context of patient visits and to display about the patient's post-handoff course. Subjects felt that a graphical display using icons was helpful to quickly identify which patients experienced important events post-handoff. Based on this input, we updated our system to its current form (Figure).

Our interviews with local patient safety leaders provided additional organization-wide considerations, including sharing learning opportunities across providers, expanding the system to support a wider range of provider types, such as medical students and the system's role in promoting a culture of safety.

\section{Lessons Learned:}

1. The EHR serves several purposes and one priority should be to facilitate ongoing provider education and calibration of decision-making.

2. Thoughtful aggregation of information and highlighting of important post-handoff events can reduce the barriers for providers to "close the loop" on prior clinical decisions.

3. Different stakeholders provide complementary perspectives when designing electronic systems focused on providing outcome feedback.

\begin{tabular}{|c|c|c|c|c|c|c|c|c|c|c|}
\hline \multicolumn{6}{|c|}{ Who is the patient? } & \multicolumn{5}{|c|}{ What happened after handoff? } \\
\hline Chart & $\begin{array}{l}\text { Sticky } \\
\text { Note }\end{array}$ & Patient ID & $\begin{array}{l}\text { When you cared } \\
\text { for patient }\end{array}$ & Dispo & $\begin{array}{l}\text { ED Discharge/ } \\
\text { Hospital Admit Dx }\end{array}$ & $\begin{array}{l}\text { Current Principal } \\
\text { Problem }\end{array}$ & Death & Code & Other & Events \\
\hline 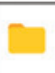 & ; & $\begin{array}{l}\text { John A. Doe } \\
79 \mathrm{M} \text { w/ altered mental status }\end{array}$ & $\begin{array}{l}11 / 24 / 1815: 20- \\
11 / 24 / 1817: 54\end{array}$ & Admit & Altered mental status & Subdural hematoma & & 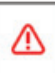 & (i) & $\begin{array}{l}\text { Stroke Code } 11 / 27 / 1816: 35 \\
\text { RRT } 11 / 27 / 1816: 37 \\
\text { Xfer Med to Surg 11/28/18 08:16 }\end{array}$ \\
\hline ron & is & $\begin{array}{l}\text { Jane A. Doe } \\
71 \mathrm{~F} w / \text { numbness }\end{array}$ & $\begin{array}{l}11 / 21 / 18 \text { 17:28 - } \\
11 / 21 / 1820: 02\end{array}$ & Admit & Headache & Intracranial mass & & & (i) & Xfer Med to Surg 11/22/18 11:19 \\
\hline ro & is & $\begin{array}{l}\text { John B. Doe } \\
54 \mathrm{M} \text { w/ SOB }\end{array}$ & $\begin{array}{l}11 / 09 / 1810: 26- \\
11 / 09 / 1816: 40\end{array}$ & Admit & Ascites & Ascites & & $\triangle$ & & RRT 11/10/18 19:00 \\
\hline r & i & $\begin{array}{l}\text { John C. Doe } \\
37 \mathrm{M} \text { w/ lump }\end{array}$ & $\begin{array}{l}11 / 21 / 1814: 25- \\
11 / 21 / 1819: 46\end{array}$ & Admit & Abscess & Neck abscess & & $\triangle$ & & RRT 11/23/18 06:05 \\
\hline r & i & $\begin{array}{l}\text { Jane B. Doe } \\
\text { 77F w/ SOB }\end{array}$ & $\begin{array}{l}11 / 21 / 1813: 18- \\
11 / 21 / 1817: 45\end{array}$ & $\begin{array}{l}\text { ED } \\
\text { Discharge }\end{array}$ & COPD exacerbation & COPD exacerbation & & & & \\
\hline r & ; & $\begin{array}{l}\text { John D. Doe } \\
28 \mathrm{M} \text { w/ tremors }\end{array}$ & $\begin{array}{l}11 / 24 / 1818: 21- \\
11 / 24 / 1823: 07\end{array}$ & $\begin{array}{l}\text { ED } \\
\text { Discharge }\end{array}$ & Alcohol withdrawal & & & & & \\
\hline 0 & i & $\begin{array}{l}\text { Jane C. Doe } \\
45 F \text { w/ hip pain }\end{array}$ & $\begin{array}{l}11 / 14 / 1819: 33- \\
11 / 14 / 1822: 17\end{array}$ & Admit & Hip pain & Pain crisis & & & & \\
\hline 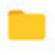 & ij & $\begin{array}{l}\text { Jane } D \text {. Doe } \\
61 \mathrm{~F} \text { / body swelling }\end{array}$ & $\begin{array}{l}11 / 24 / 1814: 46- \\
11 / 24 / 1818: 15\end{array}$ & Admit & Fatigue & Acute kidney injury & & & & \\
\hline
\end{tabular}




\title{
The Normalization of Deviance in Diagnostics
}

\author{
L. G. Keary \\ JGK Leadership Consulting
}

How often have we heard the phrase "close enough for Government work" and chuckled? While a common joke, the underlying reality is a major detriment to the ideal of achieving the highest quality possible. This presentation discusses the theory of the Normalization of Deviance and how tolerance of substandard behaviors in a variety of areas of healthcare can lead to disastrous outcomes. We start by looking at several industries which have faced and overcome this problem and use them as models for transforming healthcare into a high-performing organization which does not tolerate the normalization of deviance in our operations. The presenter will show several specific examples of how we normalize deviance in the anatomic and clinical laboratory setting and how to prevent recurrence of these failing. Finally, we will look at a specific case study of a wrongful death situation in which the normalization of deviance played a major role in the fatality.

\section{Practice Inquiry Colleague Groups: Next Phase}

\author{
L. Sommers ${ }^{1}$, A. Panchal ${ }^{2}$, S. Runyan ${ }^{3}$ and C. Forest ${ }^{4}$ \\ ${ }^{1}$ University of California, San Francisco \\ ${ }^{2}$ Tufts University Family Medicine Residency, Cambridge Health Alliance \\ Malden Family Medicine Center \\ ${ }^{3}$ One Medical, Primary Care Group Practice \\ Burlingame, California \\ ${ }^{4}$ Natividad Family Medicine Residency Program \\ Salinas, Ca
}

Primary care clinicians use advanced cognitive and communication skills to engage patients with undifferentiated, perplexing presentations. Unlike other specialists, they address the whole patient, tacitly responding to the tangle of symptoms and worries while assessing function, prioritizing concerns, and blending 'evidence' with context. Despite best efforts, diagnostic conundrums may surface and morph into diagnostic error when uncertainties go unattended. How best to support clinicians with these patients who also present with ethical, management, relationship, and prognostic dilemmas? Since 2005 in the SF Bay Area, as a CME program at University of California San Francisco through 2015, Practice Inquiry Colleague groups (PI) have provided a regularly-scheduled, facilitated, practice-based venue for physicians, nurse practitioners and physician assistants to address uncertainty cases. Now in practices nationwide, we observe PI's usefulness for realtime support through building collegiality and clinical wisdom, yet recent observations suggest new directions going forward.

In pilot form since 2001, PI has been introduced to 56 practices: 42, Northern California; 14, out-of- state. Nineteen continue in California, 7 out- of- state, 5 being residencies. Eight groups (minimum 3- year longevity) stopped largely due to facilitator departure. Eight groups have met continuously for minimum of 7 years, seven for 3 years; six out-of- state groups are in development. Starting in 2012, three regional and one national organization trained clinicians to facilitate 19 internal groups. A 10 -year analysis of 460 uncertainty cases reveals $14 \%$ presented as 'adverse events' and 50\% as diagnostic uncertainty. Published findings from self-report data show overall ratings of PI value as 4 on a 5-point scale. Facilitators observe how institutional support (e.g., food, protected time) enhances group attendance and longevity.

PI Colleague Groups have been validated by longevity, increasing clinician attendance, institutional buy-in, and high self-report satisfaction scores. Recent facilitator reports offer an interesting hypothesis: in the patient visit, clinicians address uncertainty reflexively, unaware of what they do and feel when experiencing uncertainty. Therefore, we ask, "could we observe and document level of uncertainty awareness in this complex work? In PI, could we increase awareness through new methods of uncertainty engagement expressly for the patient visit? "To explore these questions, we are embarking upon a qualitative study with selected PI participants to describe what clinicians do when they ‘don’t know.” To learn about visit-focused, uncertainty engagement, we will test methods for collecting descriptive data (e.g., I-phone templates, short checklists) and bring findings to PI groups and larger audiences.

\section{Clinical Decision Support Solution for Patients and Clinical Providers to Improve Information Flow and Reduce Diagnostic Error}

\section{$\underline{\text { V. Virani }}$}

Ada Health

Statement of Problem: Patients often struggle to correctly interpret their symptoms and the care they require. This leads some patients to seek medical care when they could have treated themselves at home, and others to underestimate their illness severity, hence delaying vital treatment. 
Physicians, especially primary care physicians, cannot know about every disease, especially with the exponential growth of available medical knowledge. These challenges lead to diagnostic errors and delayed diagnoses.

Description of the intervention: To address these issues we have developed an AI-powered symptom assessment technology that enables patients to answer several multiple choice questions about their symptom/s. The symptom assessment technology leverages a continually updated, proprietary content database of thousands of diseases and symptoms. Patients are shown a personalized assessment report suggesting their most likely conditions and most appropriate care options. The report can then be shared with physicians pre-consultation, to provide vital clinical decision support.

We tested our symptom assessment technology in a primary care clinic, over a three month period. Eligible patients completed the symptom assessment whilst waiting to see a physician, which was then shared with the physician. 15 physicians and 523 patients participated in the study.

Findings to Date: The symptom assessment technology was used by a wide range of age groups, who found it easy to use and helpful.

The symptom assessment technology has the potential to influence patient's decision making:

- $\quad 14 \%$ of participants patients would have self-cared and / or visited the local pharmacy instead of attending the clinic, if they had used the app beforehand

- $\quad 1 \%$ of participants would have attended an emergency care facility instead

Physicians found the assessment report led to improved care outcomes and efficiencies:

- In $8 \%$ of cases the symptom assessment tool suggested relevant differentials that the physician had not thought of

- In $14 \%$ of consults viewing the assessment report led to time savings, with an average of 2 minutes saved per consultation

Lessons Learned: This study highlights the potential benefits of using an AI-powered symptom assessment technology in a clinical setting to improve care outcomes, increase efficiencies and provide clinical decision support for physicians.

However, we learnt that for certain (typically common) conditions physicians saw minimal benefit from the clinical decision support. In future this study should be replicated with a focus on rare / complex diseases. Another learning was the importance of EHR integration to enable more consistent usage of the technology amongst physicians. 